Resolubilidade global para campos vetoriais no toro n-dimensional

Rafael Borro Gonzalez 



\title{
Resolubilidade global para campos vetoriais no toro n-dimensional
}

\author{
Rafael Borro Gonzalez
}

Orientador: Prof. Dr. Adalberto Panobianco Bergamasco

Coorientador: Prof. Dr. Paulo Leandro Dattori da Silva

Tese apresentada ao Instituto de Ciências Matemáticas e de Computação - ICMC-USP, como parte dos requisitos para obtenção do título de Doutor em Ciências - Matemática. VERSÃO REVISADA 
Ficha catalográfica elaborada pela Biblioteca Prof. Achille Bassi e Seção Técnica de Informática, ICMC/USP, com os dados fornecidos pelo(a) autor(a)

\begin{tabular}{|c|c|}
\hline \multirow[t]{3}{*}{ B737r } & $\begin{array}{l}\text { Borro Gonzalez, Rafael } \\
\quad \text { Resolubilidade global para campos vetoriais no } \\
\text { toro n-dimensional / Rafael Borro Gonzalez; } \\
\text { orientador Adalberto Panobianco Bergamasco; co- } \\
\text { orientador Paulo Leandro Dattori da Silva. -- São } \\
\text { Carlos, } 2015 \text {. } \\
\quad 139 \text { p. }\end{array}$ \\
\hline & $\begin{array}{l}\text { Tese (Doutorado - Programa de Pós-Graduação em } \\
\text { Matemática)-- Instituto de Ciências Matemáticas e } \\
\text { de Computação, Universidade de São Paulo, } 2015 \text {. }\end{array}$ \\
\hline & $\begin{array}{l}\text { 1. resolubilidade global. } 2 \text { campos vetoriais. } \\
\text { 3. soluçoses periódicas. I. Panobianco Bergamasco, } \\
\text { Adalberto, orient. II. Dattori da Silva, Paulo } \\
\text { Leandro, co-orient. III. Título. }\end{array}$ \\
\hline
\end{tabular}


Dedico este trabalho aos meus pais Marcos e Dora e à minha esposa Alexandra. 



\section{Agradecimentos}

Agradeço ao bom Deus, pela sua misericórdia, pelo seu amor incondicional, por me dar saúde, por me guiar e me sustentar, principalmente nos momentos difíceis; agradeço a Deus por me prosperar e por me abençoar com um trabalho que me faz feliz.

Aos meus pais, Marcos e Dora, pela dedicação e amor que tiveram na minha educação, por me ajudar e me orientar nas minhas escolhas, pelas orações e pelo apoio e incentivo nos estudos.

À minha esposa Alexandra, pela contribuição indireta neste trabalho, pelo apoio, auxílio, incentivo, pela companhia, amor e carinho constante.

Às minhas avós Levina e Janir, que contribuíram na minha educação, agradeço as orações e conselhos sábios.

Aos amigos, funcionários e professores do ICMC, onde trabalhei nos últimos 10 anos. Em especial, agradeço ao professor Adalberto Bergamasco, pelas atenciosas orientações desde 2008, pelo conhecimento transferido e pela amizade. Agradeço também ao professor Paulo Dattori, coorientador deste trabalho, pela atenção, sugestões, contribuições e pela amizade.

Agradeço muito o auxílio financeiro da FAPESP, presente desde os tempos de iniciação científica em 2007. 

Abordaremos o estudo de condições para que certas equações diferenciais parciais tenham solução. Consideraremos equações do tipo $L u=f$, onde tomamos $L$ em algumas classes de campos vetoriais em toros de dimensão maior que dois. Tais campos vetoriais são operadores que agem no espaço das funções definidas no toro e que são infinitamente diferenciáveis.

A principal questão é determinar quando tais operadores têm imagem fechada. Temos também interesse em saber quando que a imagem de tais operadores é um subespaço de codimensão finita, bem como estudar a regularidade de tais operadores.

As respostas de tais questões envolvem certas propriedades dos coeficientes desses operadores, onde citamos: a conexidade de subníveis de primitivas da parte imaginária dos coeficientes; condições Diofantinas; a ordem de anulamento dos coeficientes e relações entre as ordens de anulamento das partes real e imaginária dos coeficientes; além disso, o número de vezes que a parte imaginária de um coeficiente $c$ muda de sinal entre dois zeros consecutivos de $c$ também desempenha um papel.

Conseguimos caracterizar a resolubilidade e a hipoelíticidade global de campos vetoriais do tipo tubo em toros de dimensão maior do que dois, estendendo os resultados em dimensão dois. Depois, em dimensão três, fornecemos condições que respondem sobre a imagem ser ou não fechada, para uma outra classe de campos vetoriais que não são do tipo tubo. Uma de tais condições está relacionada com a famosa condição (P) de Nirenberg-Treves. Em particular, obtemos o mesmo para uma classe de campos vetoriais em dimen- 
são dois, para os quais a codimensão da imagem foi exaustivamente estudada. 
We are concerned with the study of properties so that we can solve certain partial differential equations. We will consider equations of the form $L u=f$, where we take $L$ in some classes of vector fields on tori of dimension greater than two. This vector fields are viewed as operators acting on the space of smooth functions defined on the torus.

The main question is to study the closedness of the range of $L$. It is also of interest to know whether the range has finite codimension, as well as to study the regularity of $L$.

The answers of these questions are connected with certain properties of the coefficients of $L$, such as: Diophantine conditions; the connectedness of some sublevel sets involving primitives of the imaginary part of the coefficients; the order of vanishing of each coefficient and relations between the order of vanishing of the real and imaginary parts of each coefficient; in addition, the number of times that the imaginary part of a coefficient $c$ changes sign between two consecutive zeros of $c$ also plays a role.

We characterize both global solvability and hypoellipticity for vector fields of tube type on tori of dimension greater than two, extending the results in dimension two. Moreover, in dimension three, we find conditions for the closedness of the range for a class of vector fields which are not of tube type. One of theese conditions is related to the well known Nirenberg-Treves condition $(\mathrm{P})$. In particular, we obtain the same for a class of vector fields on the twotorus, for which the codimension of the range was largely studied. 

1 Considerações iniciais e resultados preliminares $\quad 7$

2 Resolubilidade global para campos vetoriais do tipo tubo 19

2.I Início da demonstração do Teorema $2.6 \ldots . . . . . . .33$

2.II Segunda parte da demonstração do Teorema 2.6: caso (II) . . . . . . . . 38

2.III Final da demonstração do Teorema 2.6: caso (III) . . . . . . . . . . . . . 50

3 Hipoeliticidade global para campos vetoriais do tipo tubo $\quad 67$

3.I Demonstração do Teorema 3.1: condições necessárias . . . . . . . . . . 70

3.II Demonstração do Teorema 3.1: condições suficientes . . . . . . . . . . . . 77

4 Outro operador com imagem fechada e de codimensão infinita $\quad 81$

4.I Demonstração do Teorema $4.8 \ldots \ldots$. . . . . . . . . . . . . . . 96

4. II Demonstração do Teorema 4.10 . . . . . . . . . . . . . . . . . . 113

4.III Demonstração do Teorema 4.7 . . . . . . . . . . . . . . . . . . . . . 122

5 Considerações finais $\quad 127$

5.I Resolubilidade perto de uma órbita compacta bidimensional . . . . . . 129

$\begin{array}{ll}\text { Referências bibliográficas } & 137\end{array}$ 



\section{Introdução}

O assunto central deste trabalho é a resolubilidade global de certas classes de equações diferenciais parciais lineares formadas por campos vetoriais definidos no toro $\mathbb{T}^{n} \simeq$ $\mathbb{R}^{n} / 2 \pi \mathbb{Z}^{n}$, ou seja, operadores $\mathbb{C}$ - lineares $L: \mathcal{C}^{\infty}\left(\mathbb{T}^{n}\right) \rightarrow \mathcal{C}^{\infty}\left(\mathbb{T}^{n}\right)$ que satisfazem a regra de Leibniz $L(f g)=f L g+g L f$. Deseja-se saber para quais funções $f \in \mathcal{C}^{\infty}\left(\mathbb{T}^{n}\right)$ podemos obter uma solução da equação $L u=f$, onde buscamos $u \operatorname{em} \mathcal{C}^{\infty}\left(\mathbb{T}^{n}\right)$. É natural que apareçam condições de compatibilidade; por exemplo, para cada função $f \in \mathcal{C}^{\infty}\left(\mathbb{T}^{1}\right)$, existe $u \in \mathcal{C}^{\infty}\left(\mathbb{T}^{1}\right)$ tal que $u^{\prime}=f$ se, e somente se, $\int_{\mathbb{T}^{1}} f=0$. Outra questão é descobrir quantas condições de compatibilidade são necessárias sobre $f$ para garantir que a equação $L u=f$ tenha solução em $\mathcal{C}^{\infty}\left(\mathbb{T}^{n}\right)$, sendo que o interesse é saber se são finitas ou infinitas condições.

Dizemos que $L$ é globalmente resolúvel quando sua imagem $L \mathcal{C}^{\infty}\left(\mathbb{T}^{n}\right)$ é um subespaço fechado de $\mathcal{C}^{\infty}\left(\mathbb{T}^{n}\right)$, ou seja, quando $\left(\operatorname{ker}^{t} L\right)^{\circ}=L \mathcal{C}^{\infty}\left(\mathbb{T}^{n}\right)$, onde $\left(\operatorname{ker}^{t} L\right)^{\circ}=\{f \in$ $\mathcal{C}^{\infty}\left(\mathbb{T}^{n}\right) ;\langle\mu, f\rangle=0$, se $\left.\mu \in \operatorname{ker}^{t} L\right\}$. Em palavras, $L$ é globalmente resolúvel quando para cada $f \in\left(\operatorname{ker}^{t} L\right)^{\circ}$ é possível obter uma solução de $L u=f$ em $\mathcal{C}{ }^{\infty}\left(\mathbb{T}^{n}\right)$. O operador $L$ é dito fortemente resolúvel quando $L \mathcal{C}^{\infty}\left(\mathbb{T}^{n}\right)$ for um subespaço de codimensão finita de $\mathcal{C}^{\infty}\left(\mathbb{T}^{n}\right)$. A finitude da codimensão de $L \mathcal{C}^{\infty}\left(\mathbb{T}^{n}\right)$ está associada à regularidade do operador $L$. Dizemos que $L$ é globalmente hipoelítico quando as condições $\mu \in \mathcal{D}^{\prime}\left(\mathbb{T}^{n}\right)$ e $L \mu \in \mathcal{C}^{\infty}\left(\mathbb{T}^{n}\right)$ implicarem que $\mu \in \mathcal{C}^{\infty}\left(\mathbb{T}^{n}\right)$. Para ver trabalhos que abordam tais assuntos ou que abordam assuntos correlatos, citamos [B], [BP], [BCP], [BD1], [BD2], [BD3], [BDM], [D2], [GW], [M], [Ho] e [H3].

Embora estejamos abordando um problema de análise, veremos que certas condições geométricas, topológicas e condições de teoria dos números desempenham um papel crucial nos resultados.

Somos motivados a estudar a resolubilidade global pois os operadores em questão serão de primeira ordem e não-singulares, logo a resolubilidade local de tais operadores já é conhecida, uma vez que ela é caracterizada pela condição $(\mathcal{P})$ de Nirenberg-Treves. Um operador diferencial parcial linear de ordem $m$ e coeficientes infinitamente diferenciáveis 
em um aberto $\Omega \subset \mathbb{R}^{n}$ é um operador da forma

$$
P(x, D)=\sum_{|\alpha| \leqslant m} c_{\alpha}(x) D^{\alpha}
$$

onde $c_{\alpha} \in \mathcal{C}^{\infty}(\Omega)$ e $D^{\alpha}=(-i)^{|\alpha|} \frac{\partial^{\alpha_{1}}}{\partial x_{1}^{\alpha_{1}}} \cdots \frac{\partial^{\alpha_{n}}}{\partial x_{n}^{\alpha_{n}}}$, sendo que $\alpha=\left(\alpha_{1}, \ldots, \alpha_{n}\right) \in \mathbb{Z}_{+}^{n}$ e $|\alpha|=$ $\alpha_{1}+\ldots+\alpha_{n}$. Em uma variedade suave $\Omega$ de dimensão $n$, um operador diferencial parcial linear de ordem $m$ é um operador que em toda carta local possui uma representação como em (1). Tal operador é dito localmente resolúvel (em $\Omega$ ) se cada ponto $x \in \Omega$ possui uma vizinhança $U(x)$ em $\Omega$ tal que para toda $f \in \mathcal{C}^{\infty}(\Omega)$ existe $u \in \mathcal{D}^{\prime}(\Omega)$ solução de $L u=f$ em $U(x)$. A seguir, descrevemos a condição $(\mathcal{P})$ : suponha que $P(x, D)$ possui a expressão local

$$
P(x, D)=\sum_{|\alpha| \leqslant m} c_{\alpha}(x) D^{\alpha}
$$

e seja $p_{m}: \mathcal{T}^{*} \Omega \rightarrow \mathbb{C}$ o símbolo principal de $P(x, D)$, dado por

$$
p_{m}(x, \xi)=\sum_{|\alpha|=m} c_{\alpha}(x) \xi^{\alpha},
$$

onde $(x, \xi)$ denotam as coordenadas em $\mathcal{T}^{*} \Omega$ (ver [H5]). Dizemos que $P(x, D)$ satisfaz a condição $(\mathcal{P})$ se não existe uma função suave a valores complexos $q: \mathcal{T}^{*} \Omega \backslash\{0\} \rightarrow \mathbb{C}$ tal que $\Im\left(q p_{m}\right)$ muda de sinal ao longo de uma bicaracterística de $\Re\left(q p_{m}\right)$, ao longo da qual $q \neq 0$. Uma bicaracterística de $\Re\left(q p_{m}\right)$ é uma curva integral do campo Hamiltoniano

$$
\mathcal{H}_{\Re\left(q p_{m}\right)}=\sum_{j=1}^{n} \frac{\partial \Re\left(q p_{m}\right)}{\partial \xi_{j}} \frac{\partial}{\partial x_{j}}-\sum_{j=1}^{n} \frac{\partial \Re\left(q p_{m}\right)}{\partial x_{j}} \frac{\partial}{\partial \xi_{j}},
$$

ao longo da qual $\Re\left(q p_{m}\right) \equiv 0$ (ver [H3]). Dizemos também que uma curva é uma semibicaracterística de $p_{m}$ se ela for uma bicaracterística de $\Re\left(q p_{m}\right)$, para alguma $q \neq 0$.

A condição $(\mathcal{P})$ foi formulada pela primeira vez no artigo [NT] (1963) de Nirenberg e Treves. Após um bom número de anos e de artigos, com trabalhos dos matemáticos R. Beals e C. Fefferman-[BF], Moyer (ver [H5] e [H6]), Treves [T2] e Hörmander [H3], ficou provado que a condição $(\mathcal{P})$ é necessária e suficiente para a resolubilidade local de operadores diferenciais parciais lineares de tipo principal (ver [C]), sendo que em [H3] Hörmander mostrou que a condição $(\mathcal{P})$ implica a existência de soluções infinitamente diferenciáveis, se $f$ também for infinitamente diferenciável. 
Em contrapartida, sabe-se que a condição $(\mathcal{P})$ não é necessária para a resolubilidade global. Como consequência dos resultados desenvolvidos no presente texto, obteremos mais classes de operadores que não satisfazem a condição $(\mathcal{P})$ e que são globalmente resolúveis. Por exemplo, o operador

$$
\frac{\partial}{\partial t}+i \operatorname{sen}(t) \frac{\partial}{\partial x_{1}}+i \cos (t) \frac{\partial}{\partial x_{2}} \quad\left(\text { em } \mathbb{T}_{\left(x_{1}, x_{2}, t\right)}^{3}\right)
$$

é globalmente resolúvel (ver Exemplo 2.13), mas não satisfaz a condição $(\mathcal{P})$ (ver Lema 2.1). A condição $(\mathcal{P})$ falha pois as funções $\operatorname{sen}(t)$ e $\cos (t)$ mudam de sinal. Embora a condição $(\mathcal{P})$ não seja necessária e, portanto, tais funções possam mudar de sinal, veremos que uma condição necessária para a resolubilidade global de tais operadores envolve a quantidade de vezes que cada combinação linear $\mathbb{T}^{1} \ni t \rightarrow j \operatorname{sen}(t)+k \cos (t)$ $\left((j, k) \in \mathbb{Z}^{2}\right)$ muda de sinal, a saber, poderão ocorrer no máximo duas mudanças de sinais. Tal condição está relacionada com a conexidade dos subníveis das primitivas de cada combinação $j \operatorname{sen}(t)+k \cos (t)$ (ver Teorema 2.6 - item (II)). Por exemplo, o operador

$$
\frac{\partial}{\partial t}+\left(\operatorname{sen}^{3}(t)+i \operatorname{sen}^{3}(2 t)\right)\left(\frac{\partial}{\partial x_{1}}+\frac{\partial}{\partial x_{2}}\right)
$$

não é globalmente resolúvel (ver Exemplo 2.15), uma vez que $\operatorname{sen}^{3}(t)$ muda de sinal quatro vezes em $\mathbb{T}^{1}$.

Um outro exemplo onde vale a resolubilidade global mas não vale a condição $(\mathcal{P})$ (fora do caso tubo) é dado pelo operador $\left(\mathrm{em} \mathbb{T}_{(x, y, t)}^{3}\right)$

$$
\frac{\partial}{\partial t}+\left(\operatorname{sen}^{3}(x)+i \operatorname{sen}^{3}(2 x)\right)\left(\frac{\partial}{\partial x}+\frac{\partial}{\partial y}\right)
$$

ver Exemplo 4.14. A condição $(\mathcal{P})$ não é satisfeita pois $\operatorname{sen}^{3}(2 x)$ muda de sinal entre dois zeros consecutivos da parte real $\operatorname{sen}^{3}(x)$. Observe que $\operatorname{sen}^{3}(2 x)$ muda de sinal quatro vezes em $\mathbb{T}^{1}$. Entretanto, $\operatorname{sen}^{3}(2 x)$ muda de sinal apenas uma vez entre dois zeros consecutivos de $\operatorname{sen}^{3}(x)+i \operatorname{sen}^{3}(2 x)$; veremos que tal condição é necessária (e também suficiente) para a resolubilidade global de operadores deste tipo.

A condição $(\mathcal{P})$ também não é suficiente para que $L$ seja globalmente resolúvel; por exemplo, o operador

$$
\frac{\partial}{\partial t}+\sum_{j=1}^{N} a_{j}(t) \frac{\partial}{\partial x_{j}}
$$


satisfaz a condição $(\mathcal{P})$, pois é um operador real, mas sua resolubilidade global depende de condições Diofantinas impostas sobre os coeficientes $a_{j}$ (ver Teorema 2.6 - item (I)).

Resultados de Hörmander (ver [H3] ou [H6]) mostram que a condição $(\mathcal{P})$ implica que o operador $L$ tem imagem de codimensão finita (em particular, que $L$ é globalmente resolúvel, ver Lema 1.1) se a seguinte condição geométrica estiver satisfeita:

$(G C)$ qualquer ponto característico de $L$ pertence a um intervalo compacto de uma curva bicaracterística de $\Re(q \ell)$, sobre a qual $q \neq 0$, sem extremos característicos; onde $\ell$ denota o símbolo principal de $L$.

Sendo assim, é fundamental que trabalhemos com operadores que não satisfaçam simultaneamente as condições $(\mathcal{P})$ e $(G C)$.

Nosso objetivo central é investigar a resolubilidade das seguintes classes de operadores, as quais contêm operadores com a propriedade descrita no parágrafo acima:

$$
\text { - } L=\frac{\partial}{\partial t}+\sum_{j=1}^{N}\left(a_{j}(t)+i b_{j}(t)\right) \frac{\partial}{\partial x_{j}}, \quad\left(\mathrm{em}\left(\mathbb{T}^{N} \times \mathbb{T}^{1}\right)_{(x, t)}\right) \text {, }
$$

onde $a_{j}$ e $b_{j}$ são funções em $\mathcal{C}^{\infty}\left(\mathbb{T}^{1} ; \mathbb{R}\right)$, para cada $j=1, \ldots, N$.

$$
\text { - } \mathbf{L}=\frac{\partial}{\partial t}+(a(x)+i b(x))\left(\frac{\partial}{\partial x}+\lambda \frac{\partial}{\partial y}\right), \quad\left(\mathrm{em} \mathrm{T}_{(x, y, t)}^{3}\right)
$$

onde $a$ e $b$ são funções em $\mathcal{C}^{\infty}\left(\mathbb{T}^{1} ; \mathbb{R}\right)$ e $\lambda \in \mathbb{R}$.

$$
\text { - } \mathfrak{L}=\frac{\partial}{\partial t}+i \beta(x)\left(x^{m} \frac{\partial}{\partial x}+\frac{\partial}{\partial y}\right), \quad\left(\mathrm{em}\left((\delta, \delta) \times \mathbb{T}^{2}\right)_{(x, y, t)}\right),
$$

onde $m \geqslant 2$ e $\beta \in \mathcal{C}^{\infty}((-\delta, \delta) ; \mathbb{R})$ satisfaz $\beta(x)>0$, para todo $x \in(-\delta, \delta)$.

No Capítulo 1, apresentamos alguns conceitos e resultados gerais sobre campos vetoriais no toro, bem como estabelecemos alguns lemas que nos auxiliarão em demonstrações ao longo do texto, com o objetivo de termos demonstrações bem organizadas e com riqueza de detalhes.

O Capítulo 2 caracteriza a resolubilidade global dos operadores da forma (2) (operadores do tipo tubo), através de condições impostas sobre seus coeficientes. Dentre as propriedades dos coeficientes que desempenham papel na resolubilidade, destacamos: condições Diofantinas, a conexidade de subníveis envolvendo a parte imaginária dos co- 
eficientes e a dependência linear entre tais funções. Tal caracterização encontra-se no Teorema 2.6, que é o principal resultado do Capítulo 2.

A hipoeliticidade global dos operadores do tipo (2) também é tratada no presente trabalho. Tal estudo é feito no Capítulo 3, onde condições similares às que aparecem na resolubilidade também servem para caracterizar a hipoeliticidade. A caracterização da hipoeliticidade global de (2) é fornecida pelo Teorema 3.1, que é o principal resultado do Capítulo 3. Veremos que as condições que determinam a resolubilidade forte dessa classe de operadores são as mesmas que determinam a hipoeliticidade global. Sabemos que tal propriedade nem sempre ocorre; por exemplo, o artigo [BCP] foi pioneiro ao apresentar em $\mathbb{T}^{2}$ campos vetoriais fortemente resolúveis que não são globalmente hipoelíticos.

Os teoremas 2.6 e 3.1 estendem para várias variáveis resultados conhecidos em dimensão dois.

Dedicamos o Capítulo 4 ao estudo da resolubilidade global dos operadores do tipo (3). Quando $c^{-1}(0)=\emptyset$, tal resolubilidade é dada pelos resultados do Capítulo 2, uma vez que, através de uma mudança de variáveis, o operador (3) se reduz a um operador do tipo tubo. No caso mais interessante em que $c^{-1}(0) \neq \emptyset$, veremos que a codimensão da imagem é infinita. Sendo assim, vamos nos dedicar a encontrar condições para que a imagem seja fechada. Tais condições envolvem relações entre a ordem de anulamento das funções $a$ e $b$, bem como o número de vezes que a função $b$ muda de sinal entre dois zeros consecutivos de $c=a+i b$, a qual é intimamente relacionada com a conhecida condição $(\mathcal{P})$ de Nirenberg-Treves. O principal resultado nesta direção é o Teorema 4.7. Tal teorema fornece uma nova classe (fora do tipo tubo) de campos vetoriais que são globalmente resolúveis e não satisfazem a condição $(\mathcal{P})$. Como corolários do Teorema 4.7 , obtemos resultados que respondem se o operador $\partial_{t}+(a(x)+i b(x)) \partial_{x}$, em dimensão dois, tem ou não imagem fechada. Para tal operador, a codimensão da imagem foi exaustivamente estudada.

Por fim, no Capítulo 5 é feito um estudo sobre a resolubilidade de (4) em uma vizinhança de uma órbita compacta bidimensional. Os operadores do tipo (4) não são globalmente hipoelíticos em nenhuma vizinhança da órbita compacta bidimensional $\{0\} \times \mathbb{T}^{2}$. Entretanto, dada $f$ suave em uma vizinhança de $\{0\} \times \mathbb{T}^{2}$, basta que $f$ satisfaça um número finito de condições de compatibilidade para que exista $u$ suave em uma vizinhança de $\{0\} \times \mathbb{T}^{2}$ tal que $\mathfrak{L} u=f$. Em outras palavras, conseguimos achar um subespaço de codimensão finita de $\mathcal{C}^{\infty}\left((-\delta, \delta) \times \mathbb{T}^{2}\right)$ de modo que, qualquer que 
seja $f$ nesse espaço, a equação $\mathfrak{L} u=f$ possui uma solução suave em uma vizinhança de $\{0\} \times \mathbb{T}^{2}$. Acabamos de descrever o Teorema 5.3, que é o principal resultado do Capítulo 5 e estabelece a resolubilidade do operador (4) em um sentido correlato ao de Hörmander. 


-

\section{Considerações iniciais e resultados preliminares}

Denotaremos o toro de dimensão $n \geqslant 1$ por $\mathbb{T}^{n}=\mathbb{R}^{n} / 2 \pi \mathbb{Z}^{n}$, o qual é uma variedade suave com atlas proveniente de restrições da aplicação canônica $\mathfrak{p}: \mathbb{R}^{n} \rightarrow \mathbb{R}^{n} / 2 \pi \mathbb{Z}^{n}$. O espaço das funções $f: \mathbb{T}^{n} \rightarrow \mathbb{C}$ que são infinitamente diferenciáveis será denotado por $\mathcal{C}^{\infty}\left(\mathbb{T}^{n}\right)$. Em $\mathcal{C}^{\infty}\left(\mathbb{T}^{n}\right)$ consideraremos a topologia metrizável induzida pela sequência de seminormas

$$
|\phi|_{k} \doteq \sum_{|\alpha| \leqslant k} \sup _{q \in \mathbb{T}^{n}}\left|\left(\partial^{\alpha} \phi\right)(q)\right|
$$

onde $\phi \in \mathcal{C}^{\infty}\left(\mathbb{T}^{n}\right)$ e $k \in \mathbb{Z}_{+}$. Deste modo, $\mathcal{C}^{\infty}\left(\mathbb{T}^{n}\right)$ é um espaço vetorial topológico, localmente convexo, metrizável e completo (ver $[R]$ ), isto é, um espaço de Fréchet. O espaço dos funcionais lineares contínuos definidos em $\mathcal{C}^{\infty}\left(\mathbb{T}^{n}\right)$ será denotado por $\mathcal{D}^{\prime}\left(\mathbb{T}^{n}\right)$; os elementos de $\mathcal{D}^{\prime}\left(\mathbb{T}^{n}\right)$ são chamados de distribuições periódicas. Para um estudo sobre distribuições citamos [H5] e sobre distribuições periódicas citamos [Z] (ver também [G]).

Um campo vetorial em $\mathbb{T}^{n}$ é uma aplicação $\mathbb{C}$-linear $L: \mathcal{C}^{\infty}\left(\mathbb{T}^{n}\right) \rightarrow \mathcal{C}^{\infty}\left(\mathbb{T}^{n}\right)$ que satisfaz a regra de Leibniz $L(f g)=f L g+g L f$ (ver $[\mathrm{BCH}])$. Denotaremos por ${ }^{t} L$ o operador transposto de $L$, o qual age em $\mathcal{D}^{\prime}\left(\mathbb{T}^{n}\right)$.

Dizemos que $L$ é globalmente resolúvel quando sua imagem, $L \mathcal{C}^{\infty}\left(\mathbb{T}^{n}\right)$, é um subespaço fechado de $\mathcal{C}^{\infty}\left(\mathbb{T}^{n}\right)$. Resultados básicos de análise funcional implicam que $\left(\operatorname{ker}^{t} L\right)^{\circ}=$ $\overline{L \mathcal{C}^{\infty}\left(\mathbb{T}^{n}\right)}$ (ver $\left.[\mathrm{G}]\right)$, onde

$$
\left(\operatorname{ker}^{t} L\right)^{\circ}=\left\{u \in \mathcal{C}^{\infty}\left(\mathbb{T}^{n}\right) ;\langle\mu, u\rangle=0, \forall \mu \in \operatorname{ker}^{t} L\right\}
$$


Deste modo, $L$ é globalmente resolúvel se, e somente se, $\left(\operatorname{ker}^{t} L\right)^{\circ} \subset L \mathcal{C}^{\infty}\left(\mathbb{T}^{n}\right)$ (utilizaremos essa caracterização várias vezes ao longo deste trabalho).

Existe uma noção mais forte de resolubilidade; a saber, $L$ é fortemente resolúvel quando sua imagem é um subespaço de codimensão finita de $\mathcal{C}^{\infty}\left(\mathbb{T}^{n}\right)$ (ver $\left.[\mathrm{BCP}]\right)$. Tal definição é correlata com a definição de resolubilidade em uma vizinhança de um conjunto compacto, introduzida por Hörmander. Novamente, recorrendo à análise funcional, veremos que $L$ ser fortemente resolúvel implica que $L$ é globalmente resolúvel.

Lema 1.1. Um operador parcial linear $L$, definido em $\mathcal{C}^{\infty}\left(\mathbb{T}^{n}\right)$, é fortemente resolúvel se, e somente se, $L \mathcal{C}^{\infty}\left(\mathbb{T}^{n}\right)$ é fechado e $\operatorname{dim} \operatorname{ker}^{t} L<\infty$. Além disso, a codimensão de $L \mathcal{C}^{\infty}\left(\mathbb{T}^{n}\right)$ é igual à dimensão de $\operatorname{ker}^{t} L$.

Demonstração: Suponha que $L \mathcal{C}^{\infty}\left(\mathbb{T}^{n}\right)$ é fechado e que $\operatorname{dim} \operatorname{ker}^{t} L=m$. Como $L \mathcal{C}^{\infty}\left(\mathbb{T}^{n}\right)$ é um subespaço fechado de $\mathcal{C}^{\infty}\left(\mathbb{T}^{n}\right)$, o espaço quociente $E \doteq \mathcal{C}^{\infty}\left(\mathbb{T}^{n}\right) / L \mathcal{C}^{\infty}\left(\mathbb{T}^{n}\right)$ é um espaço vetorial topológico (Hausdorff) localmente convexo (ver $[R]$ ). Mostremos que $\operatorname{dim} E^{\prime}=m$. Seja $\mathfrak{p}: \mathcal{C}^{\infty}\left(\mathbb{T}^{n}\right) \rightarrow E$ a aplicação canônica e sejam $\mu_{1}, \ldots, \mu_{m}$ distribuições que geram $\operatorname{ker}^{t} L$. Dada $\theta \in E^{\prime}$, definimos $\mu_{\theta} \in \mathcal{D}^{\prime}\left(\mathbb{T}^{n}\right)$ por $\mu_{\theta} \doteq \theta \circ \mathfrak{p}$. Note que, para $f \in L \mathcal{C}^{\infty}\left(\mathbb{T}^{n}\right)$, temos $\left\langle\mu_{\theta}, f\right\rangle=\theta(\mathfrak{p}(f))=\theta(0)=0 ; \log 0 \mu_{\theta} \in{ }^{\circ}\left(L \mathcal{C}{ }^{\infty}\left(\mathbb{T}^{n}\right)\right)=\overline{\operatorname{ker}}^{t}{ }^{w^{*}}=$ $\operatorname{ker}^{t} L$. Como $\operatorname{ker}^{t} L$ é gerado por $\mu_{1}, \ldots, \mu_{m}$, segue que

$$
\theta(\mathfrak{p}(u))=\sum_{j=1}^{m} \lambda_{j}\left\langle\mu_{j}, u\right\rangle
$$

para toda $u \in \mathcal{C}^{\infty}\left(\mathbb{T}^{n}\right)$. Para cada $j$, definimos $\theta_{j} \in E^{\prime}$ por $\theta_{j}(\mathfrak{p}(u)) \doteq\left\langle\mu_{j}, u\right\rangle$. Como $\mu_{1}, \ldots, \mu_{m}$ são linearmente independentes, segue que $\theta_{1}, \ldots, \theta_{m}$ são linearmente independentes e como

$$
\theta=\sum_{j=1}^{m} \lambda_{j} \theta_{j}
$$

qualquer que seja $\theta \in E^{\prime}$, obtemos $\operatorname{dim} E^{\prime}=m$. Em particular, $\operatorname{dim} E=m$, ou seja, $L \mathcal{C}^{\infty}\left(\mathbb{T}^{n}\right)$ tem codimensão $m$ em $\mathcal{C}^{\infty}\left(\mathbb{T}^{n}\right)$.

Reciprocamente, suponha que $L \mathcal{C}^{\infty}\left(\mathbb{T}^{n}\right)$ tem codimensão finita $(m)$ em $\mathcal{C}^{\infty}\left(\mathbb{T}^{n}\right)$. Utilizando o Teorema da Aplicação Aberta, mostraremos que $L \mathcal{C}^{\infty}\left(\mathbb{T}^{n}\right)$ é fechado. Por hipótese, existe $S$ um subespaço de dimensão $m$ de $\mathcal{C}^{\infty}\left(\mathbb{T}^{n}\right)$ tal que $\mathcal{C}^{\infty}\left(\mathbb{T}^{n}\right)=L \mathcal{C}^{\infty}\left(\mathbb{T}^{n}\right) \oplus S$. Como $S$ é fechado e $\mathcal{C}^{\infty}\left(\mathbb{T}^{n}\right)$ é um espaço de Fréchet, segue que $S$ e $\mathcal{C}^{\infty}\left(\mathbb{T}^{n}\right) \times S$ são espaços de Fréchet. Assim, pelo Teorema da Aplicação Aberta (ver [R]) segue que a 
aplicação

$$
L \oplus i d: \mathcal{C}^{\infty}\left(\mathbb{T}^{n}\right) \times S \rightarrow \mathcal{C}^{\infty}\left(\mathbb{T}^{n}\right)
$$

definida por $L \oplus i d(u, v) \doteq L u+v$, é aberta. Em particular, $L \oplus i d\left(\mathcal{C}^{\infty}\left(\mathbb{T}^{n}\right) \backslash \operatorname{ker} L, S\right)=$ $\mathcal{C}^{\infty}\left(\mathbb{T}^{n}\right) \backslash L \mathcal{C}^{\infty}\left(\mathbb{T}^{n}\right)$ é aberto, logo $L \mathcal{C}^{\infty}\left(\mathbb{T}^{n}\right)$ é fechado em $\mathcal{C}^{\infty}\left(\mathbb{T}^{n}\right)$. Por fim, repetindo os argumentos acima vemos que $\operatorname{dim} \operatorname{ker}^{t} L=\operatorname{dim}\left[\mathcal{C}^{\infty}\left(\mathbb{T}^{n}\right) / L \mathcal{C}^{\infty}\left(\mathbb{T}^{n}\right)\right]$, que é a codimensão de $L \mathcal{C}^{\infty}\left(\mathbb{T}^{n}\right)$ em $\mathcal{C}^{\infty}\left(\mathbb{T}^{n}\right)$

Embora a resolubilidade global seja o principal assunto do presente texto, também estudaremos a hipoeliticidade global para os operadores do tipo tubo (ver (2) na Introdução). Dizemos que um operador $L: \mathcal{D}^{\prime}\left(\mathbb{T}^{n}\right) \rightarrow \mathcal{D}^{\prime}\left(\mathbb{T}^{n}\right)$ é globalmente hipoelítico se as condições $\mu \in \mathcal{D}^{\prime}\left(\mathbb{T}^{n}\right)$ e $L \mu \in \mathcal{C}^{\infty}\left(\mathbb{T}^{n}\right)$ implicam que $\mu \in \mathcal{C}^{\infty}\left(\mathbb{T}^{n}\right)$.

O próximo resultado fornece uma relação entre a hipoeliticidade global e a resolubilidade global. Sua demonstração segue a linha dos argumentos que aparecem em [H6]-Teorema 26.1.7.

Lema 1.2. Seja $L: \mathcal{C}^{\infty}\left(\mathbb{T}^{n}\right) \rightarrow \mathcal{C}^{\infty}\left(\mathbb{T}^{n}\right)$ um campo vetorial tal que ${ }^{t} L: \mathcal{D}^{\prime}\left(\mathbb{T}^{n}\right) \rightarrow \mathcal{D}^{\prime}\left(\mathbb{T}^{n}\right)$ é globalmente hipoelítico e satisfaz ${ }^{t} L=-L$. Então L é globalmente resolúvel.

Demonstração: Denotemos por $E$ o espaço $\mathcal{C}^{\infty}\left(\mathbb{T}^{n}\right)$ munido da topologia proveniente da seguinte sequência de seminormas:

$$
u \rightarrow \sup _{x \in \mathbb{T}^{n}}|u(x)|+\sum_{|\alpha| \leqslant m} \sup _{x \in \mathbb{T}^{n}}\left|\partial^{\alpha}\left({ }^{t} L u\right)(x)\right| \quad\left(m \in \mathbb{Z}_{+}\right) .
$$

Desta forma $E$ é um espaço de Fréchet. Utilizando a regra de Leibniz segue que a aplicação identidade $i d: \mathcal{C}^{\infty}\left(\mathbb{T}^{n}\right) \rightarrow E$ é contínua, logo o Teorema da Aplicação Aberta implica que $i d: E \rightarrow \mathcal{C}^{\infty}\left(\mathbb{T}^{n}\right)$ é contínua. Sendo assim, para todo $k \in \mathbb{Z}_{+}$existem $C_{k}>0$ e $m_{k} \in \mathbb{Z}_{+}$tais que

$$
\sum_{|\alpha| \leqslant k} \sup _{x \in \mathbb{T}^{n}}\left|\partial^{\alpha} u(x)\right| \leqslant C_{k}\left(\sup _{x \in \mathbb{T}^{n}}|u(x)|+\sum_{|\alpha| \leqslant m_{k}} \sup _{x \in \mathbb{T}^{n}}\left|\partial^{\alpha}\left({ }^{t} L u\right)(x)\right|\right),
$$

para toda $u \in \mathcal{C}^{\infty}\left(\mathbb{T}^{n}\right)$.

Tal fato implicará que existem $C>0$ e $m \in \mathbb{Z}_{+}$tais que

$$
\sup _{x \in \mathbb{T}^{n}}|u(x)| \leqslant C \sum_{|\alpha| \leqslant m} \sup _{x \in \mathbb{T}^{n}}\left|\partial^{\alpha}\left({ }^{t} L u\right)(x)\right|
$$


para toda $u \in\left(\operatorname{ker}^{t} L\right)^{\circ}$.

Suponha por um momento que tal condição seja verdadeira. Para cada função $f \in$ $\left(\operatorname{ker}^{t} L\right)^{\circ} \subset \mathcal{C}^{\infty}\left(\mathbb{T}^{n}\right)$, defina $T_{f}:{ }^{t} L \mathcal{C}^{\infty}\left(\mathbb{T}^{n}\right) \rightarrow \mathbb{C}$ por $\left\langle T_{f},{ }^{t} L u\right\rangle \doteq \int_{\mathbb{T}^{n}} f \cdot u$, para toda $u \in \mathcal{C}^{\infty}\left(\mathbb{T}^{n}\right)$. Note que, como $f \in\left(\operatorname{ker}^{t} L\right)^{\circ}$ e como ${ }^{t} L \mathcal{C}^{\infty}\left(\mathbb{T}^{n}\right)=L \mathcal{C}^{\infty}\left(\mathbb{T}^{n}\right) \subset\left(\operatorname{ker}^{t} L\right)^{\circ}$, segue que $T_{f}$ é bem definida; mais ainda, a estimativa (1.2) implica que $T_{f}$ é contínua. Pelo Teorema de Hahn-Banach existe $\mu \in \mathcal{D}^{\prime}\left(\mathbb{T}^{n}\right)$ tal que, para toda $u \in \mathcal{C}^{\infty}\left(\mathbb{T}^{n}\right)$, temos $\langle L \mu, u\rangle=\left\langle\mu,{ }^{t} L u\right\rangle=\left\langle T_{f},{ }^{t} L u\right\rangle=\langle f, u\rangle ; \operatorname{logo} L \mu=f$. Como $f \in \mathcal{C}^{\infty}\left(\mathbb{T}^{n}\right)$ e como $L=-{ }^{t} L$ é globalmente hipoelítico, obtemos $\mu \in \mathcal{C}^{\infty}\left(\mathbb{T}^{n}\right)$. Portanto, $L$ é globalmente resolúvel.

Para concluir a demonstração vamos provar que (1.1) implica (1.2).

Suponha que não vale (1.2). Então, para cada $\ell \in \mathbb{N}$ existe $u_{\ell} \in\left(\operatorname{ker}^{t} L\right)^{\circ}$ tal que $\sup _{x \in \mathbb{T}^{n}}\left|u_{\ell}(x)\right|=1 \mathrm{e}$

$$
\sum_{|\alpha| \leqslant \ell} \sup _{x \in \mathbb{T}^{n}}\left|\partial^{\alpha}\left({ }^{t} L u_{\ell}\right)(x)\right|<\frac{1}{\ell} .
$$

A desigualdade acima e (1.1) implicam que para todo $k \in \mathbb{Z}_{+}$existe $C_{k}>0$ tal que

$$
\sum_{|\alpha| \leqslant k} \sup _{x \in \mathbb{T}^{n}}\left|\partial^{\alpha} u_{\ell}(x)\right| \leqslant C_{k}\left(1+\sum_{|\alpha| \leqslant \ell} \sup _{x \in \mathbb{T}^{n}}\left|\partial^{\alpha}\left({ }^{t} L u_{\ell}\right)(x)\right|\right)<2 C_{k}
$$

para todo $\ell \in \mathbb{N}$ (suficientemente grande), $\operatorname{logo}\left\{u_{\ell}, \ell \in \mathbb{N}\right\}$ é limitado em $\mathcal{C}^{\infty}\left(\mathbb{T}^{n}\right)$. Como $\mathcal{C}^{\infty}\left(\mathbb{T}^{n}\right)$ tem a propriedade de Heine-Borel (todo fechado e limitado é compacto), segue que $\left(u_{\ell}\right)$ possui uma subsequência que converge para uma função $v \in \mathcal{C}^{\infty}\left(\mathbb{T}^{n}\right)$ (na topologia usual de $\left.\mathcal{C}^{\infty}\left(\mathbb{T}^{n}\right)\right)$. Mais ainda, como $\left(u_{\ell}\right) \subset\left(\operatorname{ker}^{t} L\right)^{\circ}$ e como $\sup _{x \in \mathbb{T}^{n}}\left|u_{\ell}(x)\right|=1$, segue que $v \in\left(\operatorname{ker}^{t} L\right)^{\circ}$ e $\sup _{x \in \mathbb{T}^{n}}|v(x)|=1$. Por outro lado, como $\left({ }^{t} L u_{\ell}\right)$ converge para zero (por (1.3)) e para ${ }^{t} L v$, obtemos ${ }^{t} L v=0$, ou seja $v \in\left(\operatorname{ker}^{t} L\right) \cap\left(\operatorname{ker}^{t} L\right)^{\circ}$ e $v \not \equiv 0$, o que é uma contradição.

Portanto, a desigualdade (1.2) é verdadeira.

O próximo lema é uma adaptação do Lema 6.1.2 de [H2]. Tal resultado fornece uma condição necessária para a resolubilidade global de operadores diferenciais, a qual é uma desigualdade que envolve o operador transposto. Veja também as referências [T1]-Lema II.2.1 e [H1]. 
Lema 1.3. Seja $L$ um campo vetorial globalmente resolúvel em $\mathbb{T}^{n}$. Existem constantes positivas $m\left(m \in \mathbb{Z}_{+}\right)$e $C>0$ tais que

$$
\left|\int_{\mathbb{T}^{n}} f v\right| \leqslant C \cdot\left(\sum_{|\alpha| \leqslant m} \sup _{\mathbb{T}^{n}}\left|\partial^{\alpha} f\right|\right) \cdot\left(\sum_{|\alpha| \leqslant m} \sup _{\mathbb{T}^{n}}\left|\partial^{\alpha}\left({ }^{t} L v\right)\right|\right),
$$

quaisquer que sejam $f \in\left(\operatorname{ker}^{t} L\right)^{\circ}$ e $v \in \mathcal{C}^{\infty}\left(\mathbb{T}^{n}\right)$.

Demonstração: Note que $\sum_{|\alpha| \leqslant m} \sup _{\mathbb{T}^{n}}\left|\partial^{\alpha} f\right|, m \in \mathbb{Z}_{+}$, é uma sequência separante de seminormas definidas em $\mathcal{C}^{\infty}\left(\mathbb{T}^{n}\right)$, a qual, conforme $[\mathrm{R}]$, torna $\mathcal{C}^{\infty}\left(\mathbb{T}^{n}\right)$ um espaço vetorial topológico localmente convexo e metrizável. Mais ainda, munido de tal topologia, $\mathcal{C} \infty\left(\mathbb{T}^{n}\right)$ é um espaço de Fréchet. Por outro lado, a sequência de seminormas $\sum_{|\alpha| \leqslant m} \sup _{\mathbb{T}^{n}}\left|\partial^{\alpha}\left({ }^{t} L v\right)\right|$, $m \in \mathbb{Z}_{+}$, definidas em $\mathcal{C}^{\infty}\left(\mathbb{T}^{n}\right)$, não é separante. Para contornar tal problema, consideremos o espaço quociente $\mathcal{C}^{\infty}\left(\mathbb{T}^{n}\right) / \operatorname{ker}^{t} L$. Neste espaço, a sequência $\sum_{|\alpha| \leqslant m} \sup _{\mathbb{T}^{n}}\left|\partial^{\alpha}\left({ }^{t} L v\right)\right|$, $m \in \mathbb{Z}_{+}$, é uma sequência separante de seminormas, logo $\mathcal{C}^{\infty}\left(\mathbb{T}^{n}\right) / \operatorname{ker}^{t} L$ é um espaço vetorial topológico localmente convexo e metrizável. Como $\left(\operatorname{ker}^{t} L\right)^{\circ}$ é um subespaço fechado do espaço de Fréchet $\mathcal{C}^{\infty}\left(\mathbb{T}^{n}\right)$, segue que a topologia induzida torna $\left(\operatorname{ker}^{t} L\right)^{\circ}$ um Fréchet. Consideremos no espaço produto $\left(\operatorname{ker}^{t} L\right)^{\circ} \times \mathcal{C}^{\infty}\left(\mathbb{T}^{n}\right) / \operatorname{ker}^{t} L$ a forma bilinear

$$
(f, v) \mapsto \int_{\mathbb{T}^{n}} f v
$$

a qual está bem definida, pois $f \in\left(\operatorname{ker}^{t} L\right)^{\circ}$.

Fixada $v \in \mathcal{C}^{\infty}\left(\mathbb{T}^{n}\right) / \operatorname{ker}^{t} L$, é fácil ver que a forma linear

$$
f \mapsto \int_{\mathbb{T}^{n}} f v, f \in\left(\operatorname{ker}^{t} L\right)^{\circ}
$$

é contínua em $\left(\operatorname{ker}^{t} L\right)^{\circ}$. Mais ainda, quando fixamos $f \in\left(\operatorname{ker}^{t} L\right)^{\circ}$, a resolubilidade global de $L$ implica que existe $u \in \mathcal{C}^{\infty}\left(\mathbb{T}^{n}\right)$ tal que $L u=f$; deste modo, a forma linear

$$
\mathcal{C}^{\infty}\left(\mathbb{T}^{n}\right) / \operatorname{ker}^{t} L \ni v \mapsto \int_{\mathbb{T}^{n}} f v
$$

satisfaz

$$
\left|\int_{\mathbb{T}^{n}} f v\right|=|\langle v, L u\rangle|=\left|\left\langle{ }^{t} L v, u\right\rangle\right|=\left|\int_{\mathbb{T}^{n}}\left({ }^{t} L v\right) u\right| \leqslant\left.\left|\int_{\mathbb{T}^{n}} u\right| \cdot \sup _{\mathbb{T}^{n}}\right|^{t} L v \mid,
$$


o que implica que essa segunda forma linear também é contínua.

Até aqui construímos uma forma bilinear (definida no produto de um espaço de Fréchet com um espaço vetorial topológico localmente convexo e metrizável) e mostramos que tal forma bilinear é separadamente contínua. Segue de [T3] (página 354) que tal forma é contínua, o que implica na existência de constantes $C>0$ e $m \in \mathbb{Z}_{+}$tais que

$$
\left|\int_{\mathbb{T}^{n}} f v\right| \leqslant C \cdot\left(\sum_{|\alpha| \leqslant m} \sup _{\mathbb{T}^{n}}\left|\partial^{\alpha} f\right|\right) \cdot\left(\sum_{|\alpha| \leqslant m} \sup _{\mathbb{T}^{n}}\left|\partial^{\alpha}\left({ }^{t} L v\right)\right|\right),
$$

para todo $f \in\left(\operatorname{ker}^{t} L\right)^{\circ}$ e $v \in \mathcal{C}^{\infty}\left(\mathbb{T}^{n}\right) / \operatorname{ker}^{t} L$.

Como a desigualdade é trivial para $v \in \operatorname{ker}^{t} L$, o lema está demonstrado.

A desigualdade fornecida pelo Lema 1.3 será de grande valia no estudo da resolubilidade global dos operadores do Capítulo 2.

Outra ferramenta de grande importância é a série parcial de Fourier de uma função em $\mathcal{C}^{\infty}\left(\mathbb{T}^{n}\right)$. A referência para tal assunto é $[\mathrm{Z}]$.

Sejam $p$ e $q$ dois números naturais e seja $n \doteq p+q$. Denotemos por $(x, y)$ as coordenadas em $\mathbb{T}^{n}=\mathbb{T}^{p} \times \mathbb{T}^{q}$. Uma sequência de funções $\left(v_{\eta}\right)_{\eta \in \mathbb{Z}^{q}} \subset \mathcal{C}{ }^{\infty}\left(\mathbb{T}^{p}\right)$ é rapidamente decrescente se para cada $\alpha \in \mathbb{Z}_{+}^{p}$ e $k \in \mathbb{Z}_{+}$existir $C>0$ tal que

$$
\left|\partial^{\alpha} v_{\eta}(x)\right| \leqslant C /|\eta|^{k}
$$

para todo $\eta \in \mathbb{Z}^{q} \backslash\{0\}$ e $x \in \mathbb{T}^{p}$; enquanto que $\left(\nu_{\eta}\right)_{\eta \in \mathbb{Z}^{q}} \subset \mathcal{D}^{\prime}\left(\mathbb{T}^{p}\right)$ será de crescimento lento se existirem constantes $C>0$ e $k \in \mathbb{Z}_{+}$tais que

$$
\left|\left\langle\nu_{\eta}, u\right\rangle\right| \leqslant C|\eta|^{k} \sum_{|\alpha| \leqslant k} \sup _{x \in \mathbb{T}^{p}}\left|\partial^{\alpha} u(x)\right|
$$

para todo $\eta \in \mathbb{Z}^{q} \backslash\{0\}$ e $u \in \mathcal{C}^{\infty}\left(\mathbb{T}^{p}\right)$.

Para cada função $u \in \mathcal{C}^{\infty}\left(\mathbb{T}^{n}\right)$, defina

$$
\hat{u}(x, \eta) \doteq \frac{1}{(2 \pi)^{q}} \int_{\mathbb{T}^{q}} u(x, y) e^{-i\langle\eta, y\rangle} d y
$$

para todo $x \in \mathbb{T}^{p}$ e $\eta \in \mathbb{Z}^{q}$. 
A sequência $(\hat{u}(\cdot, \eta))_{\eta \in \mathbb{Z}^{q}}$ é rapidamente decrescente e $\sum_{\eta \in \mathbb{Z}^{q}} \hat{u}(x, \eta) e^{i\langle\eta, y\rangle}$ converge para $u \operatorname{em} \mathcal{C}^{\infty}\left(\mathbb{T}^{n}\right) ;$ assim, escrevemos

$$
u(x, y)=\sum_{\eta \in \mathbb{Z}^{q}} \hat{u}(x, \eta) e^{i\langle\eta, y\rangle} .
$$

A série (1.5) é dita série parcial de Fourier da função $u$ com relação à variável $y$ e cada coeficiente $\hat{u}(\cdot, \eta)$ é chamado de coeficiente parcial de Fourier da função $u$. Reciprocamente, se $\left(v_{\eta}\right)_{\eta \in \mathbb{Z}^{q}} \subset \mathcal{C}^{\infty}\left(\mathbb{T}^{p}\right)$ é rapidamente decrescente, então a série $\sum_{\eta \in \mathbb{Z}^{q}} v_{\eta}(x) e^{i\langle\eta, y\rangle}$ converge em $\mathcal{C}^{\infty}\left(\mathbb{T}^{n}\right)$ para uma função $v$, a qual satisfaz $\hat{v}(x, \eta)=v_{\eta}(x)$.

Para $u \in \mathcal{C}^{\infty}\left(\mathbb{T}^{n}\right)$ e $x \in \mathbb{T}^{p}$, denotemos por $u_{x}$ a função em $\mathcal{C}^{\infty}\left(\mathbb{T}^{q}\right)$ dada por $u_{x}(y)=u(x, y), y \in \mathbb{T}^{q}$. Seja também $e_{\eta}(y) \doteq e^{\langle\eta, y\rangle}$, para $y \in \mathbb{T}^{q}$ e $\eta \in \mathbb{Z}^{q}$. Dadas duas distribuições $\omega \in \mathcal{D}^{\prime}\left(\mathbb{T}^{p}\right)$ e $\nu \in \mathcal{D}^{\prime}\left(\mathbb{T}^{q}\right)$, definimos $\mu=\omega \otimes \nu \in \mathcal{D}^{\prime}\left(\mathbb{T}^{n}\right)$ por $\langle\mu, u\rangle=\left\langle\omega\left\langle\nu, u_{x}\right\rangle\right\rangle$. Suponha agora que $\mu \in \mathcal{D}^{\prime}\left(\mathbb{T}^{n}\right)$. A sequência $\hat{\mu}(\cdot, \eta) \subset \mathcal{D}^{\prime}\left(\mathbb{T}^{p}\right)$, definida por $\hat{\mu}(\phi, \eta)=(2 \pi)^{-q}\left\langle\mu, \phi \cdot e_{-\eta}\right\rangle$, forma uma sequência de crescimento lento e $\sum_{\eta \in \mathbb{Z}^{q}} \hat{\mu}(\cdot, \xi) \otimes e_{\eta}$ converge em $\mathcal{D}^{\prime}\left(\mathbb{T}^{n}\right)$ para $\mu$; assim, escrevemos

$$
\mu=\sum_{\eta \in \mathbb{Z}^{q}} \hat{\mu}(\cdot, \eta) \otimes e_{\eta}
$$

ou seja

$$
\left.\langle\mu, u\rangle=\sum_{\eta \in \mathbb{Z}^{q}}\left\langle\hat{\mu}(\cdot, \eta),\left\langle e_{\eta}, u_{x}\right\rangle\right\rangle=(2 \pi)^{q} \sum_{\eta \in \mathbb{Z}^{q}}\langle\hat{\mu}(\cdot, \eta), \hat{u}(\cdot,-\eta)\rangle\right\rangle .
$$

A série (1.6) é chamada de série parcial de Fourier da distribuição $\mu$ com relação à variavel $y$ e cada coeficiente $\hat{\mu}(\cdot, \eta)$ é chamado de coeficiente parcial de Fourier da distribuição $\mu$. Por fim, se $\left(\nu_{\eta}\right)_{\eta \in \mathbb{Z}^{q}} \subset \mathcal{D}^{\prime}\left(\mathbb{T}^{p}\right)$ é uma sequência de crescimento lento, então a série $\sum_{\eta \in \mathbb{Z}^{q}} \nu_{\eta} \otimes e_{\eta}$ converge para uma distribuição $\nu$ em $\mathcal{D}^{\prime}\left(\mathbb{T}^{n}\right)$, a qual satisfaz $\left\langle\nu_{\eta}, \phi\right\rangle=(2 \pi)^{-q}\left\langle\nu, \phi \cdot e_{-\eta}\right\rangle=\hat{\nu}(\phi, \xi)$, para cada $\phi \in \mathcal{C}^{\infty}\left(\mathbb{T}^{p}\right)$ e $\eta \in \mathbb{Z}^{q}$.

Além do Lema 1.3, a série parcial de Fourier também será utilizada para buscar condições necessárias para a resolubilidade global, sendo que, nesse caso, a técnica será construir funções em $\left(\operatorname{ker}^{t} L\right)^{\circ}$ que não estejam na imagem de $L$. 
A seguir, apresentamos dois lemas que também serão utilizados para estabelecermos condições necessárias para a resolubilidade dos operadores do Capítulo 2.

Lema 1.4. Seja $\phi$ uma função em $\mathcal{C}^{\infty}\left(\mathbb{T}^{1}, \mathbb{R}\right)$ tal que $\int_{0}^{2 \pi} \phi(t) d t=0$ e tal que todos os subníveis

$$
\Omega_{r} \doteq\left\{t \in \mathbb{T}^{1} ; \int_{0}^{t} \phi(\tau) d \tau<r\right\}(r \in \mathbb{R})
$$

são conexos. Então os seguintes conjuntos são conexos:

$$
\begin{gathered}
\left\{t \in \mathbb{T}^{1}, \int_{0}^{t} \phi(\tau) d \tau \leqslant r\right\}, \quad\left\{t \in \mathbb{T}^{1}, \int_{0}^{t} \phi(\tau) d \tau \geqslant r\right\}, \\
\left\{t \in \mathbb{T}^{1}, \int_{0}^{t} \phi(\tau) d \tau=r\right\} \quad e \quad\left\{t \in \mathbb{T}^{1}, \int_{0}^{t} \phi(\tau) d \tau>r\right\},
\end{gathered}
$$

para todo $r \in \mathbb{R}$.

Demonstração: O conjunto $\left\{t \in \mathbb{T}^{1}, \int_{0}^{t} \phi(\tau) d \tau \leqslant r\right\}$ é conexo pois se escreve como a interseção de uma sequência não-crescente de conjuntos conexos $\Omega_{r+\frac{1}{m}}=\left\{t \in \mathbb{T}^{1}, \int_{0}^{t} \phi(\tau) d \tau<r+\frac{1}{m}\right\}$, ou seja,

$$
\left\{t \in \mathbb{T}^{1}, \int_{0}^{t} \phi(\tau) d \tau \leqslant r\right\}=\bigcap_{m \in \mathbb{N}} \Omega_{r+\frac{1}{m}}
$$

onde $\Omega_{r+\frac{1}{m+1}} \subset \Omega_{r+\frac{1}{m}}$, para todo $m \in \mathbb{N}$.

Os conjuntos $\left\{t \in \mathbb{T}^{1}, \int_{0}^{t} \phi(\tau) d \tau \geqslant r\right\}$ e $\left\{t \in \mathbb{T}^{1}, \int_{0}^{t} \phi(\tau) d \tau>r\right\}$ são conexos, uma vez que são o complementar $\left(e m \mathbb{T}^{1}\right)$ dos conjuntos conexos $\Omega_{r}$ e $\left\{t \in \mathbb{T}^{1}, \int_{0}^{t} \phi(\tau) d \tau \leqslant r\right\}$, respectivamente. que

Por fim, veremos que o conjunto $\left\{t \in \mathbb{T}^{1}, \int_{0}^{t} \phi(\tau) d \tau=r\right\}$ é conexo. De fato, note

$$
\left\{t \in \mathbb{T}^{1}, \int_{0}^{t} \phi(\tau) d \tau=r\right\}=\bigcap_{m \in \mathbb{N}}\left\{t \in \mathbb{T}^{1}, r-\frac{1}{m} \leqslant \int_{0}^{t} \phi(\tau) d \tau<r+\frac{1}{m}\right\}
$$

onde, para cada $m \in \mathbb{N}$, o conjunto $\left\{t \in \mathbb{T}^{1}, r-\frac{1}{m} \leqslant \int_{0}^{t} \phi(\tau) d \tau<r+\frac{1}{m}\right\}$ é conexo (pois é a interseção de dois conexos em $\mathbb{T}^{1}$ ) e vale a inclusão 


$$
\begin{aligned}
\left\{t \in \mathbb{T}^{1}, r-\frac{1}{(m+1)} \leqslant \int_{0}^{t} \phi(\tau) d \tau<r+\right. & \left.\frac{1}{(m+1)}\right\} \subset \\
& \left\{t \in \mathbb{T}^{1}, r-\frac{1}{m} \leqslant \int_{0}^{t} \phi(\tau) d \tau<r+\frac{1}{m}\right\} .
\end{aligned}
$$

Lema 1.5. Seja $\phi$ uma função em $\mathcal{C}^{\infty}\left(\mathbb{T}^{1}\right) \backslash\{0\}$ que possua uma primitiva $\Phi \in \mathcal{C}^{\infty}\left(\mathbb{T}^{1}\right)$. Suponha que exista $r \in \mathbb{R}$ tal que o subnivel $\Omega_{r}=\left\{t \in \mathbb{T}^{1} ; \Phi(t)<r\right\}$ seja desconexo. Então existe $r_{0}<r$ tal que $\Omega_{r_{0}}$ tem duas componentes conexas cujos fechos são disjuntos; desta forma, podemos definir funções $f_{0}$ e $v_{0}$ em $\mathcal{C}^{\infty}\left(\mathbb{T}^{1}\right)$, satisfazendo as seguintes propriedades: $\int_{0}^{2 \pi} f_{0}(t) d t=0, \operatorname{supp} f_{0} \cap \Omega_{r_{0}}=\emptyset, \operatorname{supp} v_{0}^{\prime} \subset \Omega_{r_{0}} e \int_{0}^{2 \pi} f_{0}(t) v_{0}(t) d t>0$.

Demonstração: Tome $C_{1}$ uma componente conexa de $\Omega_{r} \varsubsetneqq \mathbb{T}^{1}$. Note que $C_{1}$ é um intervalo aberto cuja fronteira é formada por dois pontos distintos, $\partial C_{1} \doteq\left\{t_{1}, t_{2}\right\}$. Fixando $t_{3} \in C_{1}$, temos $\Phi\left(t_{3}\right)<r$.

Como $\Omega_{r}$ não é conexo, existe $C_{2}$, outra componente conexa de $\Omega_{r}$, tal que $C_{1} \cap$ $C_{2}=\emptyset$. Novamente, $C_{2}$ é um intervalo aberto cuja fronteira é formada por dois pontos distintos, $\partial C_{2}=\left\{t_{4}, t_{5}\right\}$. Fixando $t_{6} \in C_{2}$, temos $\Phi\left(t_{6}\right)<r$.

Seja $\epsilon>0$ tal que $r_{0} \doteq \max \left\{\Phi\left(t_{3}\right), \Phi\left(t_{6}\right)\right\}+\epsilon<r$. Como $\Phi\left(t_{1}\right)=r$, segue da continuidade de $\phi$ que existe um intervalo aberto $U_{1}$ contendo $t_{1}$, tal que $\Phi(t)>r_{0}$, para todo $t \in U_{1}$. Do mesmo modo, existe um intervalo aberto $U_{2}$ contendo $t_{2}$, tal que $\Phi(t)>r_{0}$, para todo $t \in U_{2}$.

Considere $I$ e $J$, as componentes conexas de $\Omega_{r_{0}}$ que contêm $t_{3}$ e $t_{6}$, respectivamente. Note que $U_{1}$ e $U_{2}$ são subconjuntos de $\mathbb{T}^{1} \backslash(I \cup J)$. Note também que as componentes conexas $I$ e $J$ possuem fechos disjuntos.

O próximo passo é definir as funções $f_{0}$ e $v_{0}$. Para tanto, vamos olhar a situação acima em um intervalo de comprimento $2 \pi, K=\left[t_{1}, t_{1}+2 \pi\right] \subset \mathbb{R}$, onde iremos representar os conjuntos definidos até aqui do seguinte modo: assumiremos que

$$
\begin{gathered}
t_{1}<t_{3}<t_{2} \leqslant t_{4}<t_{6}<t_{5} \leqslant t_{1}+2 \pi, \\
t_{3} \in I \subset C_{1}=\left(t_{1}, t_{2}\right), \\
t_{6} \in J \subset C_{2}=\left(t_{4}, t_{5}\right),
\end{gathered}
$$




$$
U_{1}=\left[t_{1}, t_{1}+\epsilon^{\prime}\right) \cup\left(t_{1}+2 \pi-\epsilon^{\prime}, t_{1}+2 \pi\right],
$$

onde $0<\epsilon^{\prime}$ e $t_{1}+\epsilon^{\prime}<t_{3}$; por fim,

$$
U_{2}=\left(t_{2}-\epsilon^{\prime \prime}, t_{2}+\epsilon^{\prime \prime}\right)
$$

onde $0<\epsilon^{\prime \prime}$ e $t_{3}<t_{2}-\epsilon^{\prime \prime}<t_{2}+\epsilon^{\prime \prime}<t_{6}$.

Considere funções de corte $f_{1} \in \mathcal{C}_{c}^{\infty}\left(U_{1}\right)$ tal que $\int_{0}^{2 \pi} f_{1}(t) d t=1$ e $f_{2} \in \mathcal{C}_{c}^{\infty}\left(U_{2}\right)$ tal que $\int_{0}^{2 \pi} f_{2}(t) d t=1$. Definindo $f_{0} \doteq f_{2}-f_{1}$ obtemos supp $f_{0} \subset U_{1} \cup U_{2}$, o que implica $\operatorname{supp} f_{0} \cap \Omega_{r_{0}}=\emptyset$; além disso, $\int_{0}^{2 \pi} f_{0}=\int_{U_{2}} f_{2}-\int_{U_{1}} f_{1}=1-1=0$.

Por fim, seja $\delta>0$ tal que $t_{3}+\delta \in I$ e $t_{6}-\delta \in J$.

Agora escolha $v_{0}$ satisfazendo: $v_{0} \in \mathcal{C}_{c}^{\infty}\left(\left(t_{3}, t_{6}\right)\right)$ e $v_{0} \equiv 1$ em $\left[t_{3}+\delta, t_{6}-\delta\right]$; deste modo, obtemos $\int_{0}^{2 \pi} f_{0} v_{0}=\int_{U_{2}} f_{2}=1>0$ e $\operatorname{supp} v_{0}^{\prime} \subset I \cup J \subset \Omega_{r_{0}}$. O que conclui a demonstração.

A série parcial de Fourier também será empregada na busca de soluções para equações do tipo $L u=f$, a qual nos levará a uma sequência de equações envolvendo um número menor de variáveis. Precisaremos resolver tais equações, obtendo uma sequência de soluções e depois precisaremos mostrar que tal sequência decai rapidamente.

O lema abaixo nos auxiliará na resolução dessas equações. Sua demonstração é uma simples aplicação do método do fator integrante.

Lema 1.6. Sejam $f$ e $\theta$ funções em $\mathcal{C}^{\infty}\left(\mathbb{T}^{1}\right)$. Considere a equação

$$
u^{\prime}(t)+\theta(t) u(t)=f(t), \quad t \in \mathbb{T}^{1} .
$$

Se $\theta_{0} \doteq(2 \pi)^{-1} \int_{0}^{2 \pi} \theta(t) d t \notin i \mathbb{Z}$, então (1.7) possui uma única solução, a qual pode ser escrita por

$$
u(t)=\left(1-e^{-2 \pi \theta_{0}}\right)^{-1} \int_{0}^{2 \pi} f(t-s) e^{-\int_{t-s}^{t} \theta(\tau) d \tau} d s
$$

ou ainda, podemos escrever

$$
u(t)=\left(e^{2 \pi \theta_{0}}-1\right)^{-1} \int_{0}^{2 \pi} f(t+s) e^{\int_{t}^{t+s} \theta(\tau) d \tau} d s .
$$


Por outro lado, se $\theta_{0} \in i \mathbb{Z}$, então (1.7) possui infinitas soluções, desde que

$$
\int_{0}^{2 \pi} f(t) e^{\int_{0}^{t} \theta(\tau) d \tau} d t=0
$$

Neste caso, cada solução pode ser escrita na forma

$$
u(t)=C e^{-\int_{0}^{t} \theta(\tau) d \tau}+\int_{0}^{t} f(s) e^{-\int_{s}^{t} \theta(\tau) d \tau} d s, \quad \text { onde } C \in \mathbb{C} .
$$

Finalizando essa seção, apresentaremos dois lemas que nos ajudarão no controle do decaimento de certas sequências de funções.

Lema 1.7. Seja K um número real positivo. Então

$$
\int_{s_{0}-\delta}^{s_{0}+\delta} e^{-m K\left(s-s_{0}\right)^{2}} d s \geqslant\left(\int_{-\delta}^{\delta} e^{-s^{2}} d s\right)(K)^{-1 / 2}(m)^{-1 / 2}
$$

para todo $m \in \mathbb{N}$ tal que $\sqrt{m K}>1$.

Demonstração: Basta notar que

$$
\int_{s_{0}-\delta}^{s_{0}+\delta} e^{-m K\left(s-s_{0}\right)^{2}} d s=\int_{-\delta}^{\delta} e^{-(\sqrt{m K} s)^{2}} d s=\frac{1}{\sqrt{m K}} \int_{-\delta \sqrt{m K}}^{\delta \sqrt{m K}} e^{-s^{2}} d s .
$$

Lema 1.8. Seja $\alpha$ um número real negativo. A função $\phi$ dada por $\phi(x)=e^{\alpha / x}$, se $x>0$ e $\phi(x)=0$, se $x \leqslant 0$, satisfaz $\phi(x)=O\left(|x|^{m}\right)$, para todo $m \in \mathbb{Z}_{+}$.

Demonstração: É suficiente provar que $\lim _{x \rightarrow 0^{+}} \frac{e^{\alpha / x}}{x^{m}}=0$, para todo $m \in \mathbb{Z}_{+}$. Como $e^{y}>1+y$, para todo $y>0$, obtemos

$$
\begin{gathered}
e^{\frac{-\alpha}{x(m+1)}}>1+\frac{-\alpha}{x(m+1)}>\frac{-\alpha}{x(m+1)} \text {, para todo } x>0, \text { logo } \\
x^{m} e^{\frac{-\alpha}{x}}>\left(\frac{-\alpha}{x(m+1)}\right)^{m+1} x^{m}=\left(\frac{|\alpha|}{m+1}\right)^{m+1} x^{-1} .
\end{gathered}
$$

Por fim,

$$
0<\frac{e^{\alpha / x}}{x^{m}}<\left(\frac{m+1}{|\alpha|}\right)^{m+1} x \rightarrow 0, x \rightarrow 0^{+}
$$





\section{Resolubilidade global para campos vetoriais do tipo tubo}

Dedicamos este capítulo ao estudo da resolubilidade global de campos vetoriais do tipo tubo em $\mathbb{T}^{N+1}=\left(\mathbb{T}^{N} \times \mathbb{T}^{1}\right)_{(x, t)}$, ou seja, campos da forma

$$
L=\frac{\partial}{\partial t}+\sum_{j=1}^{N}\left(a_{j}(t)+i b_{j}(t)\right) \frac{\partial}{\partial x_{j}}
$$

onde $a_{j}$ e $b_{j}$ são funções em $\mathcal{C}^{\infty}\left(\mathbb{T}^{1} ; \mathbb{R}\right)$, para cada $j=1, \ldots, N$. Note que os coeficientes são funções a valores complexos, as quais dependem apenas da variável $t$; tais coeficientes serão denotados por $c_{j} \doteq a_{j}+i b_{j}$.

O objetivo é encontrar condições que digam quando que o operador (2.1) tem imagem fechada. Nesta direção, o principal resultado é o Teorema 2.6, o qual fornece uma caracterização completa para a resolubilidade global de $L$, através de propriedades impostas sobre seus coeficientes. Propriedades topológicas, geométricas e propriedades de teoria dos números aparecem no estudo da resolubilidade global de (2.1).

Note que tais operadores são não-singulares, uma vez que existe um coeficiente que nunca se anula; logo a resolubilidade local é determinada pela condição $(\mathcal{P})$ de NirenbergTreves (ver Introdução). Isso nos motiva a estudar a resolubilidade global de (2.1). Vale destacar que a condição $(\mathcal{P})$ não é necessária para a resolubilidade global de operadores do tipo (2.1). De fato, o item (II) do Teorema 2.6 fornece exemplos de operadores globalmente resolúveis que não satisfazem a condição $(\mathcal{P})$ (veja o Exemplo 2.13). 
O próximo resultado nos ajuda a entender melhor como é a condição $(\mathcal{P})$ para os operadores do tipo (2.1).

Lema 2.1. O operador $L$ dado por (2.1) satisfaz a condição (P) se, e somente se, $L$ é do tipo

$$
L=\frac{\partial}{\partial t}+\sum_{j=1}^{N}\left(a_{j}(t)+i \lambda_{j} b(t)\right) \frac{\partial}{\partial x_{j}}
$$

onde $b \in \mathcal{C}^{\infty}\left(\mathbb{T}^{1}, \mathbb{R}\right)$, b não muda de sinal e $\lambda_{j} \in \mathbb{R}, j=1, \ldots, N$.

Para demonstrar o Lema 2.1 faremos uso do seguinte resultado, o qual é uma simples variação do Lema 3.9 de $[\mathrm{K}]$.

Lema 2.2. Sejam $g$ e $h$ funções $\mathbb{R}$ - linearmente independentes em $\mathcal{C}^{\infty}\left(\mathbb{T}^{1}\right)$. Então existem inteiros não nulos $p$ e $q, p \neq q$, tais que a função $\mathbb{T}^{1} \ni t \rightarrow p g(t)+q h(t)$ muda de sinal. Além disso, se $g_{0}=\frac{1}{2 \pi} \int_{0}^{2 \pi} g(t) d t \neq 0$ ou $h_{0}=\frac{1}{2 \pi} \int_{0}^{2 \pi} h(t) d t \neq 0$, então podemos escolher os inteiros $p$ e q de modo que também tenhamos $\int_{0}^{2 \pi} p g(t)+q h(t) d t \neq 0$.

Demonstração: Essa demonstração está em [K]. Vamos repetir os argumentos aqui.

Se $g$ muda de sinal, tomamos $q=1$ e $p$ suficientemente grande de modo que $\mathbb{T}^{1} \ni t \rightarrow p g(t)+q h(t)$ muda de sinal e $\int_{0}^{2 \pi} p g(t)+q h(t) d t \neq 0$. Analogamente, obtemos $p$ e $q$ com as propriedades desejadas quando $h$ muda de sinal.

Suponha que $g$ e $h$ não mudam de sinal. Sem perda de generalidade, podemos assumir que $g(t) \geqslant 0$ e $h(t) \geqslant 0$. Defina $K=\{t \in[0,2 \pi] ; g(t)=0=h(t)\}$ e $A=[0,2 \pi] \backslash K$.

Para dois números inteiros $p$ e $q$ tais que $0 \neq p \neq q \neq 0$, podemos escrever

$p g(t)+q h(t)=(p-q) g(t)+q(g(t)+h(t))=(p-q)(g(t)+h(t))\left(\frac{g(t)}{g(t)+h(t)}+\frac{q}{p-q}\right)$

para todo $t \in A$. Assim, se encontrarmos $r=m / n \in \mathbb{Q}$ tal que a função

$$
A \ni t \rightarrow \frac{g(t)}{g(t)+h(t)}-\frac{m}{n}
$$

muda de sinal, então $t \rightarrow(m-n) g(t)+m h(t)$ também muda de sinal. Como $g$ e $h$ são $\mathbb{R}$-linearmente independentes em $\mathcal{C}^{\infty}\left(\mathbb{T}^{1}, \mathbb{R}\right)$, a função

$$
A \ni t \rightarrow \phi(t) \doteq \frac{g(t)}{g(t)+h(t)}
$$


não é constante. Sendo assim, fixemos $t_{0} \in A$ e $t_{1} \in A$ tais que $\phi\left(t_{0}\right)<\phi\left(t_{1}\right)$. Se $g_{0}+h_{0} \neq 0$, tomamos $r=m / n \in\left(\phi\left(t_{0}\right), \phi\left(t_{1}\right)\right)(m \in \mathbb{Z} \backslash\{0\}, n \in \mathbb{N})$, onde $r$ é um número racional tal que

$$
\frac{m}{n} \neq \frac{g_{0}}{g_{0}+h_{0}}
$$

caso $g_{0}+h_{0}=0$, podemos escolher quaisquer $m \in \mathbb{Z} \backslash\{0\}$ e $n \in \mathbb{N}$ tais que $r=m / n \in$ $\left(\phi\left(t_{0}\right), \phi\left(t_{1}\right)\right)$. Com essa escolha, segue de (2.2) que a função $t \rightarrow(m-n) g(t)+m h(t)$ muda de sinal; mais ainda, $\frac{1}{2 \pi} \int_{0}^{2 \pi}(m-n) g(t)+m h(t) d t \neq 0$.

Agora estamos prontos para demonstrar o Lema 2.1.

\section{Demonstração do Lema 2.1: Seja}

$$
\ell(x, t, \xi, \tau)=\tau+\left(a_{1}(t)+i b_{1}(t)\right) \xi_{1}+\cdots+\left(a_{N}(t)+i b_{N}(t)\right) \xi_{N}
$$

o símbolo principal de $L$ e

$$
\mathcal{H}_{\Re(\ell)}=\frac{\partial}{\partial t}+a_{1}(t) \frac{\partial}{\partial x_{1}}+\cdots+a_{N}(t) \frac{\partial}{\partial x_{N}}-\left[a_{1}^{\prime}(t) \xi_{1}+\cdots+a_{N}^{\prime}(t) \xi_{N}\right] \frac{\partial}{\partial \tau}
$$

o campo Hamiltoniano de $\Re(\ell)$.

Suponha que existam índices $m$ e $k$ tais que $b_{m}$ e $b_{k}$ são $\mathbb{R}$ - linearmente independentes. Sem perda de generalidade podemos assumir que $m=1$ e $k=2$. Segue do Lema 2.2 que podemos encontrar inteiros não nulos $p$ e $q$ tais que $p b_{1}+q b_{2}$ muda de sinal. Sendo assim, para $\xi(0)=(p, q, 0, \ldots, 0)$, a parte imaginária $\Im(\ell)$ muda de sinal sobre a bicaracterística $s \rightarrow\left(x(s), s, \xi(0), \tau(s)\right.$ ) de $\Re(\ell)$ (que é uma curva integral de $\mathcal{H}_{\Re(\ell)}$ ao longo da qual $\Re(\ell) \neq 0$ ), logo não vale a condição $(\mathcal{P})$.

Supondo que as funções $b_{j}(j=1, \ldots, N)$ são duas a duas $\mathbb{R}$ - linearmente dependentes, podemos escrever

$$
L=\frac{\partial}{\partial t}+\sum_{j=1}^{N}\left(a_{j}(t)+i \lambda_{j} b(t)\right) \frac{\partial}{\partial x_{j}}
$$

onde $b \in \mathcal{C}^{\infty}\left(\mathbb{T}^{1}, \mathbb{R}\right)$ e $\left\|\left(\lambda_{1}, \ldots, \lambda_{N}\right)\right\|>0$. Se $b$ muda de sinal, repetindo os argumentos acima vemos que $L$ não satisfaz a condição $(\mathcal{P})$. Por fim, suponha que $b$ não muda de sinal. Como qualquer bicaracterística de $\Re(\ell)$ é da forma $s \rightarrow(x(s), s, \xi(s), \tau(s))$, onde $\xi(s)$ é constante, segue que $\Im(\ell)$ não muda de sinal sobre as bicaracterísticas de 
$\Re(\ell)$; disso (e do Teorema 3.7 de [H4], ver também Teorema 1.0.1 de [D1]) segue que a condição $(\mathcal{P})$ está satisfeita (veja também $[\mathrm{M}]$ ).

A validade da condição $(\mathcal{P})$ também não implica que um operador do tipo $(2.1)$ seja globalmente resolúvel. Por exemplo, operadores reais com coeficientes constantes satisfazem a condição $(\mathcal{P})$, mas podem não ser globalmente resolúveis. De fato, condições Diofantinas é que determinam a resolubilidade global de tais operadores (ver exemplos em $[\mathrm{GW}]$ ou em $[\mathrm{BP}])$.

Em [GW] (1972), os autores apresentaram uma condição sobre o símbolo de um operador a coeficientes constantes $\left(e m \mathbb{T}_{\left(x_{1}, x_{2}\right)}^{2}\right)$ da forma

$$
P=\sum_{k, \ell=1}^{M} c_{k \ell}\left(-i \frac{\partial}{\partial x_{1}}\right)^{k}\left(-i \frac{\partial}{\partial x_{2}}\right)^{\ell}
$$

a qual caracteriza a hipoeliticidade global de $P$. A saber, $P$ é globalmente hipoelítico se, e somente se, existem números reais positivos $C, \gamma_{1}$ e $\gamma_{2}$ tais que

$$
\left|\sum_{k, \ell=1}^{M} c_{k \ell} n^{k} m^{\ell}\right| \geqslant C\left(n^{2}+m^{2}\right)^{-\gamma_{1}},
$$

para todo $n \in \mathbb{N}$ e $m \in \mathbb{N}$ tais que $n^{2}+m^{2}>\gamma_{2}$. Argumentos similares aos utilizados em [GW] também podem ser empregados para caracterizar a resolubilidade global (veja, por exemplo, Proposição 2.17).

Chamaremos uma condição do tipo (2.3) de condição Diofantina.

Ainda em $[\mathrm{GW}]$, quando $P=\frac{\partial}{\partial x_{1}}-\alpha \frac{\partial}{\partial x_{2}}$, a condição (2.3) fica conectada à noção de números de Liouville. Neste caso, $P$ é globalmente hipoelítico se, e somente se, $\alpha$ é um número irracional não-Liouville. Enquanto que $P$ é globalmente resolúvel se, e somente se, $\alpha$ é racional ou $\alpha$ é um número irracional não Liouville.

Definição 2.3. Dizemos que um número irracional $\alpha$ é um número de Liouville se existir uma sequência $\left(p_{n}, j_{n}\right) \in \mathbb{Z} \times \mathbb{N}$ tal que $j_{n} \rightarrow \infty$ e

$$
\left|\alpha-p_{n} / j_{n}\right|<\left(j_{n}\right)^{-n}, \quad \text { para todo } n \in \mathbb{N} \text {. }
$$

Mais adiante, veremos que condições Diofantinas também se conectam com a noção de vetores de Liouville (ver Exemplo 2.11). 
Definição 2.4. Um vetor $(\alpha, \beta) \in \mathbb{R}^{2} \backslash \mathbb{Q}^{2}$ é um vetor de Liouville se existir $C>0$ e uma sequência $\left(p_{n}, q_{n}, j_{n}\right) \in \mathbb{Z}^{2} \times \mathbb{N}$ tal que $j_{n} \geqslant 2$ e

$$
\left|\alpha-p_{n} / j_{n}\right|+\left|\beta-q_{n} / j_{n}\right|<C\left(j_{n}\right)^{-n}, \quad \text { para todo } n \in \mathbb{N} \text {. }
$$

Observação 2.5. Note que na definição de vetores de Liouville, as duas aproximações possuem o mesmo denominador. Vale também notar que quando $(\alpha, \beta) \in(\mathbb{R} \backslash \mathbb{Q})^{2}$ for um vetor de Liouville, então cada um dos números $\alpha$ e $\beta$ é um número de Liouville. Por outro lado, em [B] foi construído um par de números de Liouville $\alpha$ e $\beta$, de modo que o vetor $(\alpha, \beta)$ fosse não Liouville.

Pelo que foi descrito previamente, é natural esperar que apareçam condições Diofantinas no estudo da resolubilidade global (assim como na hipoeliticidade global) de operadores do tipo (2.1). Portanto, consideraremos as seguintes condições Diofantinas para um par $(\alpha, \beta) \in \mathbb{R}^{N} \times \mathbb{R}^{N}$ :

(DC1) existem constantes $C>0$ e $\gamma>0$ tais que

$$
|\tau+\langle\xi, \alpha+i \beta\rangle| \geqslant C(|\xi|+|\tau|)^{-\gamma}
$$

para todo $(\xi, \tau)=\left(\xi_{1}, \ldots, \xi_{N}, \tau\right) \in \mathbb{Z}^{N} \times \mathbb{Z} \backslash\{(0,0)\}$, onde $|\xi|=\left|\xi_{1}\right|+\cdots+\left|\xi_{N}\right|$.

(DC2) existem constantes $C>0$ e $\gamma>0$ tais que

$$
|\tau+\langle\xi, \alpha+i \beta\rangle| \geqslant C(|\xi|+|\tau|)^{-\gamma}
$$

para todo $(\xi, \tau) \in \mathbb{Z}^{N} \times \mathbb{Z}$ tal que $\tau+\langle\xi, \alpha+i \beta\rangle \neq 0$.

Na caracterização da resolubilidade global de $L$ utilizaremos a condição (DC2). A condição (DC1) será utilizada no estudo da hipoeliticidade global de $L$, o qual será desenvolvido no Capítulo 3. Entretanto, preferimos enunciá-la junto com a condição (DC2), uma vez que são condições bem parecidas. Note que (DC1) implica (DC2) e que $(\mathrm{DC} 1)$ e $(\mathrm{DC} 2)$ são a mesma condição para os vetores $(\alpha, \beta) \in \mathbb{R}^{N} \times \mathbb{R}^{N}$ tais que $\tau+\langle\xi, \alpha+i \beta\rangle=0$ apenas para $(\xi, \tau)=(0,0)$.

Embora a condição $(\mathcal{P})$ por si só não implique a resolubilidade global de (2.1), devemos tomar um certo cuidado, pois resultados de Hörmander (ver [H3]) fornecem uma 
condição geométrica $(G C)$ (ver Introdução) que junto com $(\mathcal{P})$ implica resolubilidade global. Felizmente, para a grande maioria dos operadores $L$ dados por (2.1), as condições $(G C)$ e $(\mathcal{P})$ não se verificam simultaneamente. De fato, se $L$ satisfaz a condição $(\mathcal{P})$, então segue do Lema 2.1 que

$$
L=\frac{\partial}{\partial t}+\sum_{j=1}^{N}\left(a_{j}(t)+i \lambda_{j} b(t)\right) \frac{\partial}{\partial x_{j}}
$$

onde $b \in \mathcal{C}^{\infty}\left(\mathbb{T}^{1}, \mathbb{R}\right)$ e $b$ não muda de sinal.

Quando $b \equiv 0$, veremos que o operador (2.4) se reduz a um operador real e com coeficientes constantes (ver Lema 2.16), cuja resolubilidade global é caracterizada pela condição Diofantina (DC2) (ver Proposição 2.17).

Suponha agora que $b \not \equiv 0$. Para que a condição $(G C)$ se verifique em $\mathbb{T}^{N+1}$, devemos mostrar que todo ponto característico pertence a um intervalo compacto de uma semibicaracterística de $\ell$, cujos pontos finais $\left(e m \mathbb{T}^{N+1}\right.$ ) são não característicos. Afirmamos que tal condição não ocorre. Seja

$$
\ell(x, t, \xi, \tau)=\tau+\left(a_{1}(t)+i \lambda_{1} b(t)\right) \xi_{1}+\cdots+\left(a_{N}(t)+i \lambda_{N} b(t)\right) \xi_{N}
$$

o símbolo principal de $L$ e denote por $C(L)$ o conjunto característico de $L$,

$$
C(L)=\{(x, t, \xi, \tau) ; \ell(x, t, \xi, \tau)=0 \text { e }(\xi, \tau) \neq(0,0)\}
$$

Defina

$$
\mathrm{N}_{2} \doteq\left\{p \in C(L) ; \mathcal{H}_{\Re(\ell)} \text { e } \mathcal{H}_{\Im(\ell)} \text { são linearmente independentes em } p\right\}
$$

onde

$$
\mathcal{H}_{\Re(\ell)}=\frac{\partial}{\partial t}+a_{1}(t) \frac{\partial}{\partial x_{1}}+\cdots+a_{N}(t) \frac{\partial}{\partial x_{N}}-\left[a_{1}^{\prime}(t) \xi_{1}+\cdots+a_{N}^{\prime}(t) \xi_{N}\right] \frac{\partial}{\partial \tau}
$$

e

$$
\mathcal{H}_{\Im(\ell)}=b(t)\left(\lambda_{1} \frac{\partial}{\partial x_{1}}+\cdots+\lambda_{N} \frac{\partial}{\partial x_{N}}\right)-b^{\prime}(t)\left[\lambda_{1} \xi_{1}+\cdots+\lambda_{N} \xi_{N}\right] \frac{\partial}{\partial \tau} .
$$

Mostremos que $\mathbf{N}_{2} \neq \emptyset$. De fato, como $b \not \equiv 0$, qualquer ponto $p=\left(x_{0}, t_{0}, \xi_{0}, \tau_{0}\right)$ do conjunto característico tal que $b\left(t_{0}\right) \neq 0$ pertence a $\mathrm{N}_{2}$. Mais ainda, segue de [H6] que 
qualquer semibicaracterística de $\ell$ por $p$ está inteiramente contida em $C(L)$. Portanto, a condição $(G C)$ não se verifica. Logo não podemos aplicar os resultados de Hörmander em [H6] para concluir a resolubilidade global de $L$.

Antes de apresentarmos o teorema principal deste capítulo, consideremos as seguintes notações, as quais serão utilizadas ao longo do presente texto:

$$
\begin{gathered}
a_{j 0} \doteq \frac{1}{2 \pi} \int_{0}^{2 \pi} a_{j}(t) d t, \quad b_{j 0} \doteq \frac{1}{2 \pi} \int_{0}^{2 \pi} b_{j}(t) d t, \quad j=1, \ldots, N \\
\alpha_{0} \doteq\left(a_{10}, \ldots, a_{N 0}\right) \in \mathbb{R}^{N}, \quad \beta_{0} \doteq\left(b_{10}, \ldots, b_{N 0}\right) \in \mathbb{R}^{N} \\
\beta(t) \doteq\left(b_{1}(t), \ldots, b_{N}(t)\right), \quad t \in \mathbb{T}^{1},
\end{gathered}
$$

onde as funções $a_{j} \in \mathcal{C}^{\infty}\left(\mathbb{T}^{1}, \mathbb{R}\right)$ e $b_{j} \in \mathcal{C}^{\infty}\left(\mathbb{T}^{1}, \mathbb{R}\right)$ formam os coeficientes do operador $L$, o qual é dado por (2.1) e recordamos ser da forma

$$
L=\frac{\partial}{\partial t}+\sum_{j=1}^{N}\left(a_{j}(t)+i b_{j}(t)\right) \frac{\partial}{\partial x_{j}}
$$

Teorema 2.6. Seja L o operador dado por (2.1).

(I) Suponha que $b_{j} \equiv 0$, para todo $j=1, \ldots, N$. Então L é globalmente resolúvel se, e somente se, o par $\left(\alpha_{0}, 0\right)$ satisfaz (DC2).

(II) Suponha que existe algum $j$ tal que $b_{j} \not \equiv 0$ e que $b_{j 0}=0$, para todo $j=1, \ldots, N$. Neste caso, L é globalmente resolúvel se, e somente se, $\left(a_{10}, \ldots, a_{N 0}\right) \in \mathbb{Z}^{N} e$ todos os subníveis $\Omega_{r}^{\xi}$ são conexos, onde

$$
\Omega_{r}^{\xi} \doteq\left\{t \in \mathbb{T}^{1} ; \int_{0}^{t}\langle\xi, \beta(\tau)\rangle d \tau<r\right\}, r \in \mathbb{R} e \xi \in \mathbb{Z}^{N}
$$

(III) $S e b_{j 0} \neq 0$ para algum $j$, então L é globalmente resolúvel se, e somente se, valem as seguintes condições:

(III.1) $\operatorname{dim} \operatorname{span}\left\{b_{1}, \ldots, b_{N}\right\}=1$;

(III.2) cada função $b_{j}$ não muda de sinal, $j=1, \ldots, N$;

(III.3) o par $\left(\alpha_{0}, \beta_{0}\right)$ satisfaz (DC2). 
A demonstração do Teorema 2.6 será feita com detalhes no presente capítulo. Antes disso, faremos algumas observações e comentários a respeito desse teorema.

Dos itens (I) e (III) no Teorema 2.6 segue o seguinte resultado sobre a resolubilidade global de campos vetoriais com coeficientes constantes.

Corolário 2.7. Considere em $\mathbb{T}^{N+1}$ o operador

$$
\frac{\partial}{\partial t}+\sum_{j=1}^{N}\left(\alpha_{j}+i \beta_{j}\right) \frac{\partial}{\partial x_{j}}
$$

onde $\alpha_{j}$ e $\beta_{j}$ são números reais, para $j=1, \ldots, N$. Tal operador é globalmente resolúvel se, e somente se, o par $(\alpha, \beta)$ satisfaz $(\mathrm{DC} 2)$, onde $\alpha=\left(\alpha_{1}, \ldots, \alpha_{N}\right)$ e $\beta=\left(\beta_{1}, \ldots, \beta_{N}\right)$.

Com a demonstração do Teorema 2.6, obteremos, em particular, uma nova demonstração para o seguinte resultado (o qual está em [Ho]-Teorema 3.2):

Teorema 2.8 ([Ho]-Teorema 3.2). Sejam a e b funções em $\mathcal{C}^{\infty}\left(\mathbb{T}^{1}, \mathbb{R}\right)$ e considere em $\mathbb{T}_{(x, t)}^{2}$ o seguinte operador

$$
L=\frac{\partial}{\partial t}+(a(t)+i b(t)) \frac{\partial}{\partial x} .
$$

Tal operador é globalmente resolúvel se, e somente se, ocorre uma das seguintes situações:

(i) $b \equiv 0$ e $a_{0}=(2 \pi)^{-1} \int_{0}^{2 \pi} a(t) d t$ não é um número de Liouville.

(ii) $b \not \equiv 0, b_{0} \doteq(2 \pi)^{-1} \int_{0}^{2 \pi} b(t) d t=0, a_{0} \in \mathbb{Z}$ e todos os subniveis

$$
\left\{t \in \mathbb{T}^{1}, \int_{0}^{t} b(\tau) d \tau<r\right\} \quad(r \in \mathbb{R})
$$

são conexos.

(iii) $b \not \equiv 0, b_{0} \neq 0$ e $b$ não muda de sinal.

É interessante notar que, em dimensão dois, quando vale a condição $(\mathcal{P})$ e o campo $L$ (dado por (2.8)) não é real (item (iii) do Teorema 2.8), a parte real do coeficiente (a função $a$ ) não desempenha papel na resolubilidade global de $L$. Por outro lado, em dimensão maior que dois, quando o campo $L$ (dado por (2.1)) não é real e vale a condição 
$(\mathcal{P})$, condições Diofantinas sobre a parte real dos coeficientes (as funções $a_{j}$ ) podem determinar se $L$ é ou não globalmente resolúvel.

Exemplo 2.9. Sejam $a$ e $b$ funções em $\mathcal{C}^{\infty}\left(\mathbb{T}^{1}, \mathbb{R}\right)$, sendo que $b$ não muda de sinal e não é identicamente nula. Considere o operador

$$
\frac{\partial}{\partial t}+(a(t)+i b(t)) \frac{\partial}{\partial x_{1}}+i b(t) \frac{\partial}{\partial x_{2}}
$$

Segue do Teorema 2.6 - item (III) que $L$ é globalmente resolúvel se, e somente se, existem constantes $C>0$ e $\gamma>0$ tais que

$$
\left|\tau+a_{0} \xi_{1}+i b_{0}\left(\xi_{1}+\xi_{2}\right)\right| \geqslant C\left(\left|\xi_{1}\right|+\left|\xi_{2}\right|+|\tau|\right)^{-\gamma}
$$

para todo $\left(\xi_{1}, \xi_{2}\right) \in \mathbb{Z}^{2}$ tal que $\tau+a_{0} \xi_{1}+i b_{0}\left(\xi_{1}+\xi_{2}\right) \neq 0$; o que ocorre se, e somente se, $a_{0}$ não é um número de Liouville (ver Definição 2.3). De fato, suponha que $a_{0}$ é um número de Liouville. Então $a_{0}$ é irracional e existe $\left(p_{n}, j_{n}\right) \in \mathbb{Z} \times \mathbb{N}$ com $j_{n} \rightarrow \infty$ e $\left|p_{n}+j_{n} a_{0}\right|<\left(j_{n}\right)^{-n+1}$. Daqui segue que (2.10) não pode valer, senão teríamos

$$
\left(j_{n}\right)^{-n+1}>\left|p_{n}+j_{n} a_{0}\right|=\left|p_{n}+j_{n} a_{0}+i b_{0}\left(j_{n}-j_{n}\right)\right| \geqslant C\left(2 j_{n}+\left|p_{n}\right|\right)^{-\gamma}
$$

e, consequentemente,

$$
1>C\left(\frac{j_{n}^{\frac{n-1}{\gamma}-1}}{2+\frac{\left|p_{n}\right|}{j_{n}}}\right)^{\gamma} \rightarrow \infty
$$

o que é uma contradição.

Reciprocamente, suponha que $a_{0}$ não é um número de Liouville. Se $a_{0}=p / q \in \mathbb{Q}$, então $\left|\tau+a_{0} \xi_{1}\right| \geqslant 1 / q$, para todo $\left(\xi_{1}, \tau\right) \in \mathbb{Z}^{2}$ tal que $\tau+a_{0} \xi_{1} \neq 0$. Se $a_{0} \in \mathbb{R} \backslash \mathbb{Q}$, então existem constantes positivas $C^{\prime}$ e $\gamma^{\prime}$ tais que $\left|\tau+a_{0} \xi_{1}\right| \geqslant C^{\prime}\left(\left|\xi_{1}\right|+|\tau|\right)^{-\gamma^{\prime}}$ para todo $\left(\xi_{1}, \tau\right) \in \mathbb{Z}^{2} \backslash\{0\}$. Note também que $\left|\xi_{1}+\xi_{2}\right| \geqslant 1$ para todo $\left(\xi_{1}, \xi_{2}\right) \in \mathbb{Z}^{2}$ tal que $\xi_{1}+\xi_{2} \neq 0$.

Sendo assim, para $\left(\xi_{1}, \xi_{2}, \tau\right) \in \mathbb{Z}^{3}$ tal que $\tau+a_{0} \xi_{1}+i b_{0}\left(\xi_{1}+\xi_{2}\right) \neq 0$ e quando $\tau+a_{0} \xi_{1} \neq 0$, obtemos

$$
\begin{gathered}
\left|\tau+a_{0} \xi_{1}+i b_{0}\left(\xi_{1}+\xi_{2}\right)\right| \geqslant\left|\tau+a_{0} \xi_{1}\right| \geqslant \min \left\{1 / q, C^{\prime}\right\}\left(\left|\xi_{1}\right|+|\tau|\right)^{-\gamma^{\prime}} \geqslant \\
\min \left\{1 / q, C^{\prime}\right\}\left(\left|\xi_{1}\right|+\left|\xi_{2}\right|+|\tau|\right)^{-\gamma^{\prime}}
\end{gathered}
$$


ou, quando $\xi_{1}+\xi_{2} \neq 0$, obtemos

$$
\left|\tau+a_{0} \xi_{1}+i b_{0}\left(\xi_{1}+\xi_{2}\right)\right| \geqslant\left|b_{0}\right|\left|\xi_{1}+\xi_{2}\right| \geqslant\left|b_{0}\right|\left(\left|\xi_{1}\right|+\left|\xi_{2}\right|+|\tau|\right)^{-\gamma^{\prime}}
$$

Portanto, vale (2.10).

Ainda considerando a situação (III) no Teorema 2.6, faremos mais exemplos que mostram a bonita relação entre a resolubilidade global e a noção de números e vetores de Liouville (ver definições 2.3 e 2.4).

Exemplo 2.10. Seja $b \in \mathcal{C}^{\infty}\left(\mathbb{T}^{1}, \mathbb{R}\right) \backslash\{0\}$ uma função que não muda de sinal e sejam $\lambda_{1}$ e $\lambda_{2}$ números reais. O Teorema 2.6 implica que o operador

$$
\frac{\partial}{\partial t}+i b(t)\left(\lambda_{1} \frac{\partial}{\partial x_{1}}+\lambda_{2} \frac{\partial}{\partial x_{2}}\right)
$$

é globalmente resolúvel se, e somente se, existem constantes $C>0$ e $\gamma>0$ tais que

$$
\left|\tau+i b_{0}\left(\xi_{1} \lambda_{1}+\xi_{2} \lambda_{2}\right)\right| \geqslant C\left(\left|\xi_{1}\right|+\left|\xi_{2}\right|+|\tau|\right)^{-\gamma}
$$

para todo $\left(\xi_{1}, \xi_{2}, \tau\right) \in \mathbb{Z}^{3}$ tal que $\tau+i b_{0}\left(\xi_{1} \lambda_{1}+\xi_{2} \lambda_{2}\right) \neq 0$. Argumentando como no Exemplo 2.9 vemos que vale (2.12) se, e somente se, $\lambda_{1} \cdot \lambda_{2}=0$ ou $\lambda_{1} / \lambda_{2}$ não é um número de Liouville.

Exemplo 2.11. Sejam $a \in \mathcal{C}^{\infty}\left(\mathbb{T}^{1}, \mathbb{R}\right)$ e $b \in \mathcal{C}^{\infty}\left(\mathbb{T}^{1}, \mathbb{R}\right) \backslash\{0\}$, onde $b$ não muda de sinal e sejam $\lambda_{1}$ e $\lambda_{2}$ dois números reais. Considere o operador

$$
\frac{\partial}{\partial t}+\left(a(t)+i \lambda_{1} b(t)\right) \frac{\partial}{\partial x_{1}}+i \lambda_{2} b(t) \frac{\partial}{\partial x_{2}}
$$

Afirmamos que o operador (2.13) é globalmente resolúvel se, e somente se, ocorre uma das seguintes condições:

- $\lambda_{1}=\lambda_{2}=0$ e $a_{0}$ não é um número de Liouville.

- $\lambda_{1} \neq 0$ e $\lambda_{2}=0$.

- $\lambda_{2} \neq 0$ e $\left(a_{0}, \lambda_{1} / \lambda_{2}\right)$ não é um vetor de Liouville. 
Do Teorema 2.6, item (III), segue que (2.13) é globalmente resolúvel se, e somente se, existem $C_{1}>0$ e $\gamma>0$ tais que

$$
\left|\tau+a_{0} \xi_{1}+i b_{0}\left(\lambda_{1} \xi_{1}+\lambda_{2} \xi_{2}\right)\right| \geqslant C_{1}\left(\left|\xi_{1}\right|+\left|\xi_{2}\right|+|\tau|\right)^{-\gamma}
$$

para todo $\left(\xi_{1}, \xi_{2}, \tau\right) \in \mathbb{Z}^{3}$ tal que $\tau+a_{0} \xi_{1}+i b_{0}\left(\lambda_{1} \xi_{1}+\lambda_{2} \xi_{2}\right) \neq 0$.

Os casos em que $\lambda_{1}=\lambda_{2}=0$ ou $\lambda_{1} \neq 0$ e $\lambda_{2}=0$ seguem raciocinando como no Exemplo 2.9.

Suponha agora que $\lambda_{2} \neq 0$ e que $\left(a_{0}, \lambda_{1} / \lambda_{2}\right)$ é um vetor de Liouville. Então existe $C_{2}>0$ e $\left(p_{n}, q_{n}, j_{n}\right) \in \mathbb{Z}^{2} \times \mathbb{N}$ tal que $j_{n} \geqslant 2$ e

$$
\left|j_{n} a_{0}-p_{n}\right|+\left|j_{n}\left(\lambda_{1} / \lambda_{2}\right)-q_{n}\right|<C_{2}\left(j_{n}\right)^{1-n}
$$

para todo $n \in \mathbb{N}$. Como

$$
\left[\left|j_{n} a_{0}-p_{n}\right|+\left|j_{n}\left(\lambda_{1} / \lambda_{2}\right)-q_{n}\right|\right] \max \left\{1,\left|b_{0} \lambda_{2}\right|\right\} \geqslant\left|-p_{n}+j_{n} a_{0}+i b_{0} \lambda_{2}\left[j_{n}\left(\lambda_{1} / \lambda_{2}\right)-q_{n}\right]\right|
$$

e como $-p_{n}+j_{n} a_{0}+i b_{0} \lambda_{2}\left[j_{n}\left(\lambda_{1} / \lambda_{2}\right)-q_{n}\right] \neq 0$, pois $a_{0}$ ou $\lambda_{1} / \lambda_{2}$ é irracional, segue que o par $\left(\left(a_{0}, 0\right), b_{0}\left(\lambda_{1}, \lambda_{2}\right)\right)$ não satisfaz (2.14). Portanto, (2.13) não é globalmente resolúvel (Teorema 2.6).

Reciprocamente, suponha que $\left(a_{0}, \lambda_{1} / \lambda_{2}\right)$ não é um vetor de Liouville. Se tivermos $\left(a_{0}, \lambda_{1} / \lambda_{2}\right) \in \mathbb{Q}^{2}$, então repetindo novamente argumentos do Exemplo 2.9 obtemos a validade de (2.14), logo (2.13) é globalmente resolúvel. Suponha agora que $\left(a_{0}, \lambda_{1} / \lambda_{2}\right) \in$ $\mathbb{R}^{2} \backslash \mathbb{Q}^{2}$. Então existem constantes $C_{3}>0$ e $\gamma^{\prime}>0$ tais que

$$
\left|\tau+\xi_{1} a_{0}\right|+\left|\xi_{1}\left(\lambda_{1} / \lambda_{2}\right)+\xi_{2}\right| \geqslant C_{3}\left(\left|\xi_{1}\right|+\left|\xi_{2}\right|+|\tau|\right)^{-\gamma^{\prime}}
$$

para todo $\left(\xi_{1}, \xi_{2}, \tau\right) \in \mathbb{Z}^{3} \backslash\{0\}$; logo a condição (2.14) está satisfeita e , portanto, (2.13) é globalmente resolúvel.

É interessante notar que, no Exemplo 2.11, embora os campos vetoriais $\partial_{t}+(a(t)+$ $\left.i \lambda_{1} b(t)\right) \partial_{x_{1}}$ e $\partial_{t}+i \lambda_{2} b(t) \partial_{x_{2}}$ (considerados como campos vetoriais em $\mathbb{T}^{2}$ ) sejam globalmente resolúveis, pode ocorrer perda de resolubilidade ao formarmos o operador (2.13).

Os próximos exemplos ilustrarão as condições que aparecem no item (II) do Teorema 2.6. Para tanto, observemos primeiro o seguinte resultado: 
Lema 2.12. Sejam $\ell$ e $m$ inteiros tais que $1 \leqslant \ell \leqslant m \leqslant N$. Denote as coordenadas em $\mathbb{T}^{m-\ell+2}$ por $(x, t)$, onde $x=\left(x_{\ell}, \ldots, x_{m}\right) \in \mathbb{T}^{m-\ell+1}$ e $t \in \mathbb{T}^{1}$. Considere o operador $L_{\ell m}$ (definido em $\left.\mathcal{C}^{\infty}\left(\mathbb{T}^{m-\ell+2}\right)\right)$ dado por

$$
L_{\ell m}=\partial_{t}+\sum_{j=\ell}^{m} c_{j}(t) \frac{\partial}{\partial x_{j}} \quad\left(c_{j} \in \mathcal{C}^{\infty}\left(\mathbb{T}^{1}\right)\right)
$$

Se o operador $L_{\ell m}$ não é globalmente resolúvel, então

$$
L=\partial_{t}+\sum_{j=1}^{N} c_{j}(t) \frac{\partial}{\partial x_{j}} \quad\left(c_{j} \in \mathcal{C}^{\infty}\left(\mathbb{T}^{1}\right)\right)
$$

também não é globalmente resolúvel (note que L é definido em $\mathcal{C}^{\infty}\left(\mathbb{T}^{N+1}\right)$ ).

Demonstração: Como o operador $L_{\ell m}$ não é globalmente resolúvel, existe uma função $f=f\left(x_{\ell}, \ldots, x_{m}, t\right) \in\left(\operatorname{ker}^{t} L_{\ell m}\right)^{\circ} \backslash L_{\ell m} \mathcal{C}^{\infty}\left(\mathbb{T}^{m-\ell+2}\right)$. Utilizando série parcial de Fourier com relação às variáveis $\left(x_{1}, \ldots, x_{\ell-1}, x_{m+1}, \ldots, x_{N}\right)$ verifica-se que essa mesma função $f$ pertence a $\left(\operatorname{ker}^{t} L\right)^{\circ} \backslash L \mathcal{C}^{\infty}\left(\mathbb{T}^{N+1}\right)$.

Do Lema 2.12 e do Teorema 2.8 segue que se existir um subnível

$$
\left\{t \in \mathbb{T}^{1} ; \int_{0}^{t} b_{j}(\tau) d \tau<r\right\} \quad(r \in \mathbb{R}, j \in\{1, \ldots, N\})
$$

que seja desconexo, então $L$ não será globalmente resolúvel. Por outro lado, suponha que tais subníveis sejam todos conexos. Será que isso é suficiente para termos todos os subníveis $\Omega_{r}^{\xi}$ conexos? Lembre-se que $\beta(\tau)=\left(b_{1}(\tau), \ldots, b_{N}(\tau)\right)$ e

$$
\Omega_{r}^{\xi} \doteq\left\{t \in \mathbb{T}^{1} ; \int_{0}^{t}\langle\xi, \beta(\tau)\rangle d \tau<r\right\} \quad\left(r \in \mathbb{R}, \xi \in \mathbb{Z}^{N}\right)
$$

Veremos que, dependendo das funções $b_{j}$, a resposta para tal questionamento pode ser afirmativa ou negativa.

Exemplo 2.13. Sejam $b_{1}(t)=\operatorname{sen} t$ e $b_{2}(t)=\cos t, t \in \mathbb{T}^{1}$. Mostremos que todos os subníveis

$$
\left\{t \in \mathbb{T}^{1}, \int_{0}^{t} j \operatorname{sen}(\tau)+k \cos (\tau) d \tau<r\right\} \quad\left(r \in \mathbb{R},(j, k) \in \mathbb{Z}^{2}\right)
$$


são conexos.

Inicialmente, note que

$$
\left\{t \in \mathbb{T}^{1}, \int_{0}^{t} j \operatorname{sen}(\tau)+k \cos (\tau) d \tau<r\right\}=\left\{t \in \mathbb{T}^{1},-j \cos (t)+j+k \operatorname{sen}(t)<r\right\}
$$

e defina $\phi(t)=-j \cos (t)+j+k \operatorname{sen}(t), t \in \mathbb{T}^{1}$.

Suponha que não estamos no caso trivial em que $j=k=0$. Como $\phi^{\prime}(t)=j \operatorname{sen}(t)+$ $k \cos (t)$, analisando os zeros das funções $\operatorname{sen}(t)$ e $\cos (t)$ concluímos que $\phi^{\prime}(t)=0$ para exatamente dois pontos distintos $t_{1} \in \mathbb{T}^{1}$ e $t_{2} \in \mathbb{T}^{1}$. Mais ainda, considerando a segunda derivada $\phi^{\prime \prime}(t)=j \cos (t)-k \operatorname{sen}(t)$ e dependendo dos sinais de $j$ e $k$, vê-se que um dos pontos é ponto de máximo de $\phi$, enquanto que o outro é ponto de mínimo de $\phi$. Veremos que essas propriedades da função $\phi(t)$ implicam que todos os subníveis $\left\{t \in \mathbb{T}^{1}, \phi(t)<r\right\}$ $(r \in \mathbb{R})$ são conexos. De fato, suponha por contradição que exista um subnível $\Omega \doteq\{t \in$ $\left.\mathbb{T}^{1}, \phi(t)<r_{1}\right\}$ que seja desconexo e sejam $A$ e $B$ duas componentes conexas distintas de $\Omega$. Como $\Omega \subset \mathbb{T}^{1}$ é aberto, $A$ e $B$ são arcos abertos de $\mathbb{T}^{1}$. Mais ainda, as fronteiras $\partial A$ e $\partial B$ são subconjuntos de $\mathbb{T}^{1} \backslash \Omega$. Assim, $\phi(t)=r_{1}$ para $t \in \partial A \cup \partial B$; logo o mínimo de $\phi$ em $\bar{A}$ pertence a $A$ e o mínimo de $\phi$ em $\bar{B}$ pertence a $B$. Isso implica que $\phi^{\prime}$ tem dois zeros distintos, os quais são mínimos locais de $\phi$, o que é uma contradição.

Portanto, todos os subníveis

$$
\left\{t \in \mathbb{T}^{1}, \int_{0}^{t} j \operatorname{sen}(s)+k \cos (s) d s<r\right\}
$$

são conexos. O item (II) do Teorema 2.6 implica que o operador

$$
\frac{\partial}{\partial t}+i \operatorname{sen}(t) \frac{\partial}{\partial x_{1}}+i \cos (t) \frac{\partial}{\partial x_{2}}
$$

é globalmente resolúvel.

Exemplo 2.14. Mostremos que existem funções $b_{1} \in \mathcal{C}^{\infty}\left(\mathbb{T}^{1}, \mathbb{R}\right)$ e $b_{2} \in \mathcal{C}^{\infty}\left(\mathbb{T}^{1}, \mathbb{R}\right)$ tais que todos os subníveis

$$
\left\{t \in \mathbb{T}^{1}, \int_{0}^{t} b_{1}(\tau) d \tau<r\right\} \quad \text { e } \quad\left\{t \in \mathbb{T}^{1}, \int_{0}^{t} b_{2}(\tau) d \tau<r\right\}
$$


são conexos (para todo $r \in \mathbb{R}$ ), mas algum subnível

$$
\left\{t \in \mathbb{T}^{1}, \int_{0}^{t} b_{1}(\tau)+b_{2}(\tau) d \tau<r\right\}
$$

é desconexo. De fato, tome $\psi \in \mathcal{C}_{c}^{\infty}(\mathbb{R}, \mathbb{R})$ uma função tal que $\psi(t) \geqslant 0$, supp $\psi \subset(-\delta, \delta)$, onde $0<\delta<\pi / 4$, e $\int_{-\delta}^{\delta} \psi(t) d t=1$. Considere $b_{1}(t)=\psi(t-\pi / 4)-\psi(t-3 \pi / 4) \mathrm{e}$ $b_{2}(t)=\psi(t-5 \pi / 4)-\psi(t-7 \pi / 4), t \in \mathbb{R}$. Para tais funções, o subnível

$$
\left\{t \in \mathbb{T}^{1} ; \int_{0}^{t} b_{1}(\tau)+b_{2}(\tau) d \tau<1 / 2\right\}
$$

é desconexo. Portanto, do Teorema 2.6, item (II), segue que o operador

$$
\frac{\partial}{\partial t}+i b_{1}(t) \frac{\partial}{\partial x_{1}}+i b_{2}(t) \frac{\partial}{\partial x_{2}}
$$

não é globalmente resolúvel.

Exemplo 2.15. Um operador menos abstrato que ilustre o caso em que a resolubilidade global não ocorre, na situação (II) do Teorema 2.6, é o seguinte:

$$
\frac{\partial}{\partial t}+\left(\operatorname{sen}^{3}(t)+i \operatorname{sen}^{3}(2 t)\right)\left(\frac{\partial}{\partial x_{1}}+\frac{\partial}{\partial x_{2}}\right) .
$$

Note que $\int_{0}^{2 \pi} \operatorname{sen}^{3}(2 t) d t=0$ e considere $\theta(t)=\int_{0}^{t} \operatorname{sen}^{3}(2 \tau) d \tau$. Mostremos que existe um subnível $\left\{t \in \mathbb{T}^{1} ; \theta(t)<r\right\}$ que é desconexo. A primeira derivada $\theta^{\prime}(t)=\operatorname{sen}^{3}(2 t)$ é positiva em $(0, \pi / 2) \cup(\pi, 3 \pi / 2)$, negativa em $(\pi / 2, \pi) \cup(3 \pi / 2,2 \pi)$ e em $[0,2 \pi)$ se anula em $\{0, \pi / 2, \pi, 3 \pi / 2\}$. Assim, a função $\theta$ possui as seguintes propriedades: é estritamente crescente em $(0, \pi / 2) \cup(\pi, 3 \pi / 2)$; possui máximos em $\pi / 2$ e $3 \pi / 2$; é estritamente decrescente em $(\pi / 2, \pi) \cup(3 \pi / 2,2 \pi)$; possui mínimos em 0 e $\pi$. Como $\theta(\pi / 2)=\theta(3 \pi / 2)=$ $2 / 3>0$, qualquer subnível $\left\{t \in \mathbb{T}^{1} ; \theta(t)<r\right\}$ com $0<r<2 / 3$ é desconexo. Do Teorema 2.6, item (II), segue que (2.15) não é globalmente resolúvel.

Concluímos aqui as observações e comentários a respeito do Teorema 2.6. Estamos prontos para abordar a sua demonstração. 


\section{I Início da demonstração do Teorema 2.6}

O objetivo agora é organizar a demonstração do Teorema 2.6, o qual caracteriza a resolubilidade global de $L$ (dado por (2.1)) através de propriedades a serem satisfeitas por seus coeficientes. Tal caracterização abrange todas as possibilidades, no sentido de que dado um operador do tipo $L$, sabemos dizer se $L$ é ou não globalmente resolúvel. Como vários casos são tratados pelo Teorema 2.6, é natural organizar sua demonstração dividindo-a em proposições e lemas. Faremos também uma divisão em seções.

Nesta seção apresentaremos uma conjugação que nos permitirá supor que a parte real dos coeficientes de $L$ são constantes (ver Lema 2.16). Tal conjugação será utilizada tanto na resolubilidade quanto na hipoeliticidade de $L$. Além disso, na presente seção abordaremos, com a Proposição 2.17, o caso (I) no Teorema 2.6.

A situação (II) será tratada na Seção 2.II. As proposições 2.18 e 2.19 mostram que as condições impostas sobre os coeficientes são necessárias para a resolubilidade de $L$, enquanto que a Proposição 2.20 cuida de mostrar que tais condições são suficientes.

Por fim, lidaremos com a situação (III) na Seção 2.III. As proposição 2.21 e 2.22 mostram que as condições (III.1) e (III.2), respectivamente, são condições necessárias para a resolubilidade global de $L$. Supondo que (III.1) e (III.2) estão satisfeitas, a Proposição 2.24 prova que (III.3) também é uma condição necessária para a resolubilidade global de L. Por fim, a Proposição 2.25 mostra que as condições (III.1)-(III.3) implicam que $L$ é globalmente resolúvel, concluindo a demonstração do Teorema 2.6.

Uma das principais ferramentas utilizadas na demonstração do Teorema 2.6 é a série parcial de Fourier com relação às variáveis $\left(x_{1}, \ldots, x_{N}\right)$, a qual nos permite escrever qualquer função $\phi$ em $\mathcal{C}^{\infty}\left(\mathbb{T}^{N+1}\right)$ na seguinte forma:

$$
\phi(x, t)=\sum_{\xi \in \mathbb{Z}^{N}} \hat{\phi}(\xi, t) e^{i\langle\xi, x\rangle} .
$$

De fato, suponha que queremos mostrar que o operador $L$ (dado por (2.1)) é globalmente resolúvel. Para tanto, basta mostrar a inclusão $\left(\operatorname{ker}^{t} L\right)^{\circ} \subset L \mathcal{C}{ }^{\infty}\left(\mathbb{T}^{N+1}\right)$, ou seja, dada $f \in\left(\operatorname{ker}^{t} L\right)^{\circ}$, queremos exibir $u \in \mathcal{C}^{\infty}\left(\mathbb{T}^{N+1}\right)$ tal que $L u=f$. A série parcial de Fourier aparece na hora de buscarmos tal solução $u$, de modo que tal busca se transforme 
na procura de uma sequência de soluções de edo's do tipo

$$
L_{\xi} \hat{u}(\xi, t) \doteq \partial_{t} \hat{u}(\xi, t)+i\langle\xi, \alpha(t)+i \beta(t)\rangle \hat{u}(\xi, t)=\hat{f}(\xi, t), \quad t \in \mathbb{T}^{1}, \quad \xi \in \mathbb{Z}^{N},
$$

onde $\alpha(t)=\left(a_{1}(t), \ldots, a_{N}(t)\right)$ e $\beta(t)=\left(b_{1}(t), \ldots, b_{N}(t)\right)$. Será importante fixar cada solução $\hat{u}(\xi, \cdot) \in \mathcal{C}^{\infty}\left(\mathbb{T}^{1}\right)$ de modo que possamos obter, a partir da sequência $\hat{u}(\xi, \cdot)$, uma função $u \in \mathcal{C}^{\infty}\left(\mathbb{T}^{N+1}\right)$, que seja solução de $L u=f$. Para tanto, basta que $\hat{u}(\xi, \cdot)$ seja rapidamente decrescente (ver Capítulo 1). Note que a busca de soluções para cada equação (2.16) envolve o Lema 1.6.

Por outro lado, quando desejamos provar que $L$ não é globalmente resolúvel, também podemos utilizar série parcial de Fourier para construir uma função $f$ em $\left(\operatorname{ker}^{t} L\right)^{\circ} \backslash$ $L \mathcal{C}^{\infty}\left(\mathbb{T}^{N+1}\right)$. Outro argumento padrão na busca por condições necessárias para a resolubilidade global de $L$ é utilizar o Lema 1.3. Essas duas técnicas irão aparecer durante a demonstração do Teorema 2.6.

Inicialmente, veremos que é possível reduzir $L$ a um campo cuja parte real de cada coeficiente $c_{j}$ é constante igual a $a_{j 0}=(2 \pi)^{-1} \int_{0}^{2 \pi} a_{j}(t) d t$, para cada $j=1, \ldots, N$. Mais ainda, quando $a_{j 0}$ for um número inteiro, podemos substituí-lo por zero.

Lema 2.16. Seja L o operador dado por (2.1). Existe uma conjugação $T: \mathcal{C}^{\infty}\left(\mathbb{T}^{N+1}\right) \rightarrow$ $\mathcal{C}^{\infty}\left(\mathbb{T}^{N+1}\right)$ tal que

$$
T L T^{-1}=\frac{\partial}{\partial t}+\sum_{j=1}^{N}\left(a_{j 0}+i b_{j}(t)\right) \frac{\partial}{\partial x_{j}},
$$

onde podemos substituir $a_{j 0}$ por zero sempre que $a_{j 0} \in \mathbb{Z}$. Em particular, o operador (2.1) é globalmente resolúvel (hipoelítico) se, e somente se, o operador (2.17) é globalmente resolúvel (hipoelítico).

Demonstração: Utilizaremos série parcial de Fourier com relação às variáveis $\left(x_{1}, \ldots, x_{N}\right)$ para construir a conjugação $T: \mathcal{C}^{\infty}\left(\mathbb{T}^{N+1}\right) \rightarrow \mathcal{C}^{\infty}\left(\mathbb{T}^{N+1}\right)$. Dada

$$
u(x, t)=\sum_{\xi \in \mathbb{Z}^{N}} \hat{u}(\xi, t) e^{i\langle\xi, x\rangle}
$$

defina

$$
\widehat{T u}(\xi, t) \doteq \hat{u}(\xi, t) \exp \left\{i \int_{0}^{t}\left\langle\xi, \alpha(\tau)-\alpha_{0}\right\rangle d \tau\right\}, \quad \text { para todo } \xi \in \mathbb{Z}^{N}
$$


Cálculos diretos mostram que o decaimento rápido de $\hat{u}(\xi, \cdot)$ implica o decaimento rápido de $\widehat{T u}(\xi, t)$, logo podemos definir

$$
T u(x, t) \doteq \sum_{\xi \in \mathbb{Z}^{N}} \widehat{T u}(\xi, t) e^{i\langle\xi, x\rangle} .
$$

Analogamente, definimos a inversa de $T, T^{-1}: \mathcal{C}^{\infty}\left(\mathbb{T}^{N+1}\right) \rightarrow \mathcal{C}^{\infty}\left(\mathbb{T}^{N+1}\right)$, a qual é dada por

$$
T^{-1} v(x, t) \doteq \sum_{\xi \in \mathbb{Z}^{N}} \widehat{T^{-1} v}(\xi, t) e^{i\langle\xi, x\rangle}
$$

onde

$$
\widehat{T^{-1} v}(\xi, t) \doteq \hat{v}(\xi, t) \exp \left\{-i \int_{0}^{t}\left\langle\xi, \alpha(\tau)-\alpha_{0}\right\rangle d \tau\right\}, \quad \text { para todo } \quad \xi \in \mathbb{Z}^{N}
$$

Cálculos diretos mostram que

$$
T L T^{-1}=\frac{\partial}{\partial t}+\sum_{j=1}^{N}\left(a_{j 0}+i b_{j}(t)\right) \frac{\partial}{\partial x_{j}},
$$

onde agora a parte real de cada coeficiente é constante. Mais ainda, analisando as fórmulas das definições de $T$ e $T^{-1}$, verifica-se que podemos substituir $a_{j 0}$ por zero sempre que $a_{j 0}$ é um inteiro.

Daqui por diante, assumiremos que o operador $L$ é da forma (2.17).

O primeiro caso que consideramos na demonstração do Teorema 2.6 é quando $b_{j} \equiv 0$, para todo $j=1, \ldots, N$. Neste caso, o operador $L$ passa a ser um operador real e com coeficientes constantes,

$$
L=\frac{\partial}{\partial t}+\sum_{j=1}^{N} a_{j 0} \frac{\partial}{\partial x_{j}} .
$$

Veremos que tal operador é globalmente resolúvel se, e somente se, o par $\left(\alpha_{0}, 0\right)$ (onde $\alpha_{0}=\left(a_{10}, \ldots, a_{N 0}\right)$ ) satisfaz a condição Diofantina (DC2), o que cuida do caso (I) no Teorema 2.6. A técnica utilizada para demonstrar tal resultado é uma pequena adaptação dos argumentos que aparecem em [GW]. 
Proposição 2.17. O operador L dado por (2.18) é globalmente resolúvel se, e somente se, $\left(\alpha_{0}, 0\right)$ satisfaz (DC2), ou seja, existem constantes $C>0$ e $\gamma>0$ tais que

$$
\left|\tau+\left\langle\xi, \alpha_{0}\right\rangle\right| \geqslant C(|\xi|+|\tau|)^{-\gamma}, \quad \text { para todo }(\xi, \tau) \in \mathbb{Z}^{N} \times \mathbb{Z} \text { tal que } \tau+\left\langle\xi, \alpha_{0}\right\rangle \neq 0
$$

Demonstração: Essa demonstração é inspirada por argumentos de [GW].

Suponha que $\left(\alpha_{0}, 0\right)$ satisfaz (DC2), ou seja, existem constantes $C>0$ e $\gamma>0$ tais que

$$
\left|\tau+\left\langle\xi, \alpha_{0}\right\rangle\right| \geqslant C(|\xi|+|\tau|)^{-\gamma}
$$

para todo $(\xi, \tau) \in \mathbb{Z}^{N} \times \mathbb{Z}$ tal que $\tau+\left\langle\xi, \alpha_{0}\right\rangle \neq 0$.

Dada $f \in\left(\operatorname{ker}^{t} L\right)^{\circ}$, vamos exibir uma solução de $L u=f$ em $\mathcal{C}^{\infty}\left(\mathbb{T}^{N+1}\right)$. Para tanto, escrevemos $f$ e $u$ utilizando série de Fourier,

$$
f(x, t)=\sum_{(\xi, \tau) \in \mathbb{Z}^{N} \times \mathbb{Z}} \hat{f}(\xi, \tau) e^{i(\langle\xi, x\rangle+\tau t)}
$$

e

$$
u(x, t)=\sum_{(\xi, \tau) \in \mathbb{Z}^{N} \times \mathbb{Z}} \hat{u}(\xi, \tau) e^{i(\langle\xi, x\rangle+\tau t)} .
$$

Deste modo, a equação $L u=f$ implica que

$$
i\left(\tau+\left\langle\xi, \alpha_{0}\right\rangle\right) \hat{u}(\xi, \tau)=\hat{f}(\xi, \tau)
$$

para cada $(\xi, \tau) \in \mathbb{Z}^{N} \times \mathbb{Z}$

Para $(\xi, \tau) \in \mathbb{Z}^{N} \times \mathbb{Z}$, a função

$$
\phi_{(\xi, \tau)}(x, t) \doteq e^{-i(\langle\xi, x\rangle+\tau t)}
$$

está em $\mathcal{C}^{\infty}\left(\mathbb{T}^{N+1}\right)$. Mais ainda, se $(\xi, \tau)$ é tal que $\tau+\left\langle\xi, \alpha_{0}\right\rangle=0$, então um cálculo direto mostra que $-{ }^{t} L \phi_{(\xi, \tau)}=L \phi_{(\xi, \tau)}=-i\left(\tau+\left\langle\xi, \alpha_{0}\right\rangle\right) \phi_{(\xi, \tau)}=0$. Como $f \in\left(\operatorname{ker}^{t} L\right)^{\circ}$, obtemos

$$
0=\left\langle\phi_{(\xi, \tau)}, f\right\rangle=\int_{\mathbb{T}^{N+1}} f(x, t) e^{-i(\langle\xi, x\rangle+\tau t)} d x d t=(2 \pi)^{N+1} \hat{f}(\xi, \tau)=0
$$


Sendo assim, podemos fixar qualquer constante como solução de (2.20), fixemos $\hat{u}(\xi, \tau)=0$.

Por outro lado, se $(\xi, \tau) \in \mathbb{Z}^{N} \times \mathbb{Z}$ e $\tau+\left\langle\xi, \alpha_{0}\right\rangle \neq 0$, então

$$
\hat{u}(\xi, \tau)=\hat{f}(\xi, \tau)\left(\tau+\left\langle\xi, \alpha_{0}\right\rangle\right)^{-1}
$$

é a única solução de (2.20).

Por fim, a estimativa (2.19) e o decaimento rápido de $\hat{f}(\xi, \tau)$ implicam que $\hat{u}(\xi, \tau)$ decai rapidamente. Assim, a fórmula

$$
u(x, t)=\sum_{(\xi, \tau) \in \mathbb{Z}^{N} \times \mathbb{Z}} \hat{u}(\xi, \tau) e^{i(\langle\xi, x\rangle+\tau t)}
$$

define uma função $u \in \mathcal{C}^{\infty}\left(\mathbb{T}^{N+1}\right)$, a qual é solução de $L u=f$.

Reciprocamente, suponha que o par $\left(\alpha_{0}, 0\right)$ não satisfaz (DC2). Vamos construir uma função em $\left(\operatorname{ker}^{t} L\right)^{\circ} \backslash L \mathcal{C}^{\infty}\left(\mathbb{T}^{N+1}\right)$. Como não vale $(\mathrm{DC} 2)$, existe uma sequência $\left(\xi(n), \tau_{n}\right)_{n \in \mathbb{N}}$ tal que $|\xi(n+1)|+\left|\tau_{n+1}\right|>|\xi(n)|+\left|\tau_{n}\right| \geqslant n, \tau_{n}+\left\langle\xi(n), \alpha_{0}\right\rangle \neq 0 \mathrm{e}$

$$
\left|\tau_{n}+\left\langle\xi(n), \alpha_{0}\right\rangle\right|<\left(|\xi(n)|+\left|\tau_{n}\right|\right)^{-n}
$$

para todo $n \in \mathbb{N}$.

Como a sequência $\left(|\xi(n)|+\left|\tau_{n}\right|\right)^{-n / 2}$ é rapidamente decrescente, podemos definir uma função $f$ em $\mathcal{C}^{\infty}\left(\mathbb{T}^{N+1}\right)$ por

$$
\hat{f}(x, t)=\sum_{n=1}^{\infty}\left(|\xi(n)|+\left|\tau_{n}\right|\right)^{-n / 2} e^{i\left(\langle\xi(n), x\rangle+\tau_{n} t\right)} .
$$

Afirmamos que tal $f$ pertence ao conjunto $\left(\operatorname{ker}^{t} L\right)^{\circ} \backslash L \mathcal{C}^{\infty}\left(\mathbb{T}^{N+1}\right)$. De fato, se $\mu \in$ $\mathcal{D}^{\prime}\left(\mathbb{T}^{N+1}\right)$ e ${ }^{t} L \mu=-L \mu=0$, então os coeficientes de Fourier $(\hat{\mu}(\xi, \tau))$ de $\mu$ satisfazem

$$
i\left(\tau+\left\langle\xi, \alpha_{0}\right\rangle\right) \hat{\mu}(\xi, \tau)=0
$$

em particular,

$$
-i\left(\tau_{n}+\left\langle\xi(n), \alpha_{0}\right\rangle\right) \hat{\mu}\left(-\xi(n),-\tau_{n}\right)=0, \text { para todo } n \in \mathbb{N} .
$$


Como $\tau_{n}+\left\langle\xi(n), \alpha_{0}\right\rangle \neq 0$, obtemos $\hat{\mu}\left(-\xi(n),-\tau_{n}\right)=0$, para todo $n \in \mathbb{N}$. Assim,

$$
\langle\mu, f\rangle=\sum_{n=1}^{\infty}(2 \pi)^{N+1}\left(|\xi(n)|+\left|\tau_{n}\right|\right)^{-n / 2} \hat{\mu}\left(-\xi(n),-\tau_{n}\right)=0
$$

qualquer que seja a distribuição $\mu \in \operatorname{ker}^{t} L$. Portanto, $f \in\left(\operatorname{ker}^{t} L\right)^{\circ}$.

Por fim, suponha por contradição que existe $u \in \mathcal{C}^{\infty}\left(\mathbb{T}^{N+1}\right)$ tal que $L u=f$. Então os coeficientes de Fourier de $u, \hat{u}\left(\xi(n), \tau_{n}\right)$, satisfazem

$$
i\left(\tau_{n}+\left\langle\xi(n), \alpha_{0}\right\rangle\right) \hat{u}\left(\xi(n), \tau_{n}\right)=\left(|\xi(n)|+\left|\tau_{n}\right|\right)^{-n / 2}
$$

Sendo assim,

$$
\hat{u}\left(\xi(n), \tau_{n}\right)=-i\left(\tau_{n}+\left\langle\xi(n), \alpha_{0}\right\rangle\right)^{-1}\left(|\xi(n)|+\left|\tau_{n}\right|\right)^{-n / 2},
$$

logo (2.22) implica que

$$
\left|\hat{u}\left(\xi(n), \tau_{n}\right)\right|>\left|\xi(n)+\tau_{n}\right|^{n / 2} \geqslant n^{n / 2}
$$

o que é uma contradição, pois $\lim _{n \rightarrow \infty}\left|\hat{\mu}\left(\xi(n), \tau_{n}\right)\right|=0$, já que $\hat{\mu}\left(\xi(n), \tau_{n}\right)$ decai rapidamente.

\section{II Segunda parte da demonstração do Teorema 2.6: caso (II)}

O próximo passo na demonstração do Teorema 2.6 é considerar o caso (II), onde $b_{j} \not \equiv$ 0 , para pelo menos um $j \in\{1, \ldots, N\}$ e $b_{j 0}=0$, para todo $j=1, \ldots, N$. Começaremos mostrando que as condições impostas sobre os coeficientes de $L$ são necessárias para a resolubilidade global.

Proposição 2.18. Considere L o operador definido em (2.17). Suponha que $b_{j 0}=0$, para todo $j$, e que pelo menos uma das funções $b_{j}$ não se anula identicamente. Nestas condições, se $\left(a_{10}, \ldots, a_{N 0}\right) \notin \mathbb{Z}^{N}$, então L não é globalmente resolúvel.

Demonstração: A demonstração é motivada por argumentos que aparecem em [B]. 
Por hipótese, existem $m$ e $\ell$ em $\{1, \ldots, N\}$ tais que: $b_{m} \not \equiv 0, b_{m 0}=0$ e $a_{\ell 0} \notin \mathbb{Z}$.

Suponha primeiro que podemos tomar $m=\ell$. Sendo assim, temos $b_{m} \not \equiv 0, b_{m 0}=0$ e $a_{m 0} \notin \mathbb{Z}$. A menos de uma mudaça de variáveis, a qual é apenas uma reordenação das variáveis $x_{1}, \ldots, x_{N}$, podemos assumir que $m=1$.

Vamos utilizar tais informações para construir, por meio da série parcial de Fourier com relação às variáveis $\left(x_{1}, \ldots, x_{N}\right)$, uma função $f \in\left(\operatorname{ker}^{t} L\right)^{\circ} \backslash L \mathcal{C}^{\infty}\left(\mathbb{T}^{N+1}\right)$, a qual será da forma

$$
f\left(x_{1}, \ldots, x_{N}, t\right)=\sum_{\xi \in \mathbb{Z}^{N}} \hat{f}(\xi, t) e^{i\langle\xi, x\rangle},
$$

onde os coeficientes parciais de Fourier $\hat{f}(\xi, t)$ serão definidos posteriormente. Como $a_{10} \notin \mathbb{Z}$, existe uma sequência crescente de inteiros positivos $k_{n}>n$, tal que $k_{n} a_{10} \notin \mathbb{Z}$, para todo $n \in \mathbb{N}$. Considere também a função

$$
H(s, t) \doteq \int_{t-s}^{t} b_{1}(\tau) d \tau, \quad 0 \leqslant s, t \leqslant 2 \pi
$$

e defina

$$
A \doteq H\left(s_{0}, t_{0}\right)=\max _{0 \leqslant s, t \leqslant 2 \pi} H(s, t)
$$

Note que $A>0$, pois $b_{1}$ deve mudar de sinal; logo $0<s_{0}<2 \pi$. Daí, fazendo uma mudança de variáveis, a qual é uma translação em $t$, podemos assumir que $0<t_{0}, s_{0}, t_{0}-$ $s_{0}<2 \pi$; defina $\sigma_{0}=t_{0}-s_{0}$.

Tome $\phi \in \mathcal{C}_{c}^{\infty}\left(\left(\sigma_{0}-\delta, \sigma_{0}+\delta\right)\right)$ uma função que satisfaz $0 \leqslant \phi(t) \leqslant 1$ (para todo $t)$ e $\phi(t) \equiv 1$ em uma vizinhança de $\left[\sigma_{0}-\delta / 2, \sigma_{0}+\delta / 2\right]$, onde $\delta>0$ é suficientemente pequeno de modo que $\left(\sigma_{0}-\delta, \sigma_{0}+\delta\right) \subset\left(0, t_{0}\right)$.

Estamos prontos para definir os coeficientes de Fourier da função $f$. Para $\xi=\xi(n) \doteq$ $\left(k_{n}, 0, \ldots, 0\right) \in \mathbb{Z}^{N}$, tome $\hat{f}(\xi(n), t)$ como sendo a extensão $2 \pi$-periódica da função

$$
\phi(t) e^{-A k_{n}} e^{i k_{n} a_{10}\left(t_{0}-t\right)}, \quad t \in[0,2 \pi]
$$

Para os demais $\xi \in \mathbb{Z}^{N}$, defina $\hat{f}(\xi, t) \equiv 0$. Como $A k_{n}>A n>0$, a sequência assim definida decresce rapidamente, logo a expressão

$$
f\left(x_{1}, \ldots, x_{N}, t\right)=\sum_{n=1}^{\infty} \hat{f}(\xi(n), t) e^{i k_{n} x_{1}}
$$


define uma função em $\mathcal{C}^{\infty}\left(\mathbb{T}^{N+1}\right)$ (note que $f$ depende apenas das variáveis $x_{1}$ e $t$ ).

Afirmamos que $f \in\left(\operatorname{ker}^{t} L\right)^{\circ}$. De fato, se $\mu \in \mathcal{D}^{\prime}\left(\mathbb{T}^{N+1}\right)$ e satisfaz ${ }^{t} L \mu=0$, então a série parcial de Fourier de $\mu$ com relação às variáveis $\left(x_{1}, \ldots, x_{N}\right)$ leva-nos às equações

$$
\partial_{t} \hat{\mu}(-\xi(n), t)-i k_{n}\left(a_{10}+i b_{1}(t)\right) \hat{\mu}(-\xi(n), t)=0, \quad n \in \mathbb{N} .
$$

Como $k_{n} a_{10} \notin \mathbb{Z},(2.23)$ e o Lema 1.6 implicam que $\hat{\mu}(-\xi(n), t) \equiv 0$, para todo $n \in \mathbb{N}$. Em particular,

$$
\langle\mu, f\rangle=\sum_{n=1}^{\infty}(2 \pi)^{N}\langle\hat{\mu}(-\xi(n), t), \hat{f}(\xi(n), t)\rangle=0,
$$

e, portanto, $f \in\left(\operatorname{ker}^{t} L\right)^{\circ}$.

Para concluir esse primeiro caso, basta mostrar que $f \notin L \mathcal{C}^{\infty}\left(\mathbb{T}^{N+1}\right)$. Suponha, por contradição, que existe $u \in \mathcal{C}^{\infty}\left(\mathbb{T}^{N+1}\right)$ tal que $L u=f$. Novamente, utilizando série parcial de Fourier com relação às variáveis $\left(x_{1}, \ldots, x_{N}\right)$, segue que cada coeficiente $\hat{u}(\xi(n), \cdot)$ satisfaz a equação

$$
\partial_{t} \hat{u}(\xi(n), t)+i k_{n}\left(a_{10}+i b_{1}(t)\right) \hat{u}(\xi(n), t)=\hat{f}(\xi(n), t), \quad n \in \mathbb{N} \quad e \quad t \in \mathbb{T}^{1}
$$

Como $k_{n} a_{10} \notin \mathbb{Z}$, o Lema 1.6 implica que cada equação (2.24) possui uma única solução, a qual pode ser escrita na forma

$$
\begin{aligned}
\hat{u}(\xi(n), t) & =\left(1-e^{-2 \pi i k_{n} a_{10}}\right)^{-1} \int_{0}^{2 \pi} \hat{f}(\xi(n), t-s) \exp \left\{k_{n} \int_{t-s}^{t} b_{1}(\tau) d \tau\right\} e^{-i s k_{n} a_{10}} d s \\
& =\left(1-e^{-2 \pi i k_{n} a_{10}}\right)^{-1} \int_{0}^{2 \pi} \hat{f}(\xi(n), t-s) e^{k_{n}\left(H(s, t)-i s a_{10}\right)} d s .
\end{aligned}
$$

A definição de $\hat{f}(\xi(n), \cdot)$ implica que

$$
\begin{aligned}
\hat{u}\left(\xi(n), t_{0}\right) & =\left(1-e^{-2 \pi i k_{n} a_{10}}\right)^{-1}\left\{\int_{0}^{s_{0}-\delta}+\int_{s_{0}-\delta}^{s_{0}+\delta}+\int_{s_{0}+\delta}^{2 \pi}\right\} \hat{f}\left(\xi(n), t_{0}-s\right) e^{k_{n}\left(H\left(s, t_{0}\right)-i s a_{10}\right)} d s \\
& =\left(1-e^{-2 \pi i k_{n} a_{10}}\right)^{-1} \int_{s_{0}-\delta}^{s_{0}+\delta} \hat{f}\left(\xi(n), t_{0}-s\right) e^{k_{n}\left(H\left(s, t_{0}\right)-i s a_{10}\right)} d s \\
& =\left(1-e^{-2 \pi i k_{n} a_{10}}\right)^{-1} \int_{s_{0}-\delta}^{s_{0}+\delta} \phi\left(t_{0}-s\right) e^{-k_{n}\left(A-H\left(s, t_{0}\right)\right)} d s
\end{aligned}
$$


$\log 0$

$$
\begin{aligned}
\left|\hat{u}\left(\xi(n), t_{0}\right)\right| & =\left|1-e^{-2 \pi i k_{n} a_{10}}\right|^{-1} \int_{s_{0}-\delta}^{s_{0}+\delta} \phi\left(t_{0}-s\right) e^{-k_{n}\left[A-H\left(s, t_{0}\right)\right]} d s \\
& \geqslant \frac{1}{2} \int_{s_{0}-\delta / 2}^{s_{0}+\delta / 2} e^{-k_{n}\left[A-H\left(s, t_{0}\right)\right]} d s .
\end{aligned}
$$

Agora vamos estimar a integral em (2.26). Para tanto, defina $\psi(s)=A-H\left(s, t_{0}\right)$, $s \in\left(s_{0}-\delta / 2, s_{0}+\delta / 2\right)$. Note que $\psi(s) \geqslant 0$ e que $\psi\left(s_{0}\right)=0$, logo $s_{0}$ é um ponto de mínimo de $\psi$. Assim, $s_{0}$ é um zero de ordem infinita ou $s_{0}$ é um zero de ordem par de $\psi$. Em particular, $s_{0}$ é um zero de ordem $\geqslant 2$, logo podemos utilizar fórmula de Taylor com resto integral para escrever

$$
\psi(s)=\left(s-s_{0}\right)^{2} \int_{0}^{1}(1-\tau) \psi^{\prime \prime}\left(s_{0}+\tau\left(s-s_{0}\right)\right) d \tau
$$

para todo $s \in\left(s_{0}-\delta / 2, s_{0}+\delta / 2\right)$. Desta forma, existe uma contante $K>0$ tal que

$$
|\psi(s)| \leqslant K\left(s-s_{0}\right)^{2}, \quad \text { para todo } \quad s \in\left(s_{0}-\delta / 2, s_{0}+\delta / 2\right) .
$$

De (2.26) e (2.27) obtemos

$$
\left|\hat{u}\left(\xi(n), t_{0}\right)\right| \geqslant \frac{1}{2} \int_{s_{0}-\delta / 2}^{s_{0}+\delta / 2} e^{-k_{n} \psi(s)} d s \geqslant \frac{1}{2} \int_{s_{0}-\delta / 2}^{s_{0}+\delta / 2} e^{-k_{n} K\left(s-s_{0}\right)^{2}} d s .
$$

Aplicando o Lema 1.7 temos

$$
\left|\hat{u}\left(\xi(n), t_{0}\right)\right| \geqslant\left(\int_{-\delta_{0}}^{\delta_{0}} e^{-s^{2}} d s\right)(K)^{-1 / 2}\left(k_{n}\right)^{-1 / 2}
$$

para todo $n \in \mathbb{N}$ suficientemente grande. Desta forma, temos $\lim _{n \rightarrow \infty}|\xi(n)|\left|\hat{u}\left(\xi(n), t_{0}\right)\right|=$ $\lim _{n \rightarrow \infty} k_{n}\left|\hat{u}\left(\xi(n), t_{0}\right)\right|=\infty$; o que é uma contradição, pois $\hat{u}(\xi(n), t)$ decai rapidamente.

Para finalizar essa demonstração falta analisar o caso em que $m \neq \ell$. Novamente, a menos de uma reordenação das variáveis $x_{1}, \ldots, x_{N}$, podemos assumir que $m=1 \mathrm{e}$ $\ell=2$.

Seguindo o mesmo raciocínio do caso anterior, vamos utilizar série parcial de Fourier com relação às variáveis $\left(x_{1}, \ldots, x_{N}\right)$, para construir uma função $g \in\left(\operatorname{ker}^{t} L\right)^{\circ} \backslash$ $L \mathcal{C}^{\infty}\left(\mathbb{T}^{N+1}\right)$. Neste caso, temos $b_{1} \not \equiv 0, b_{10}=0, a_{10} \in \mathbb{Z}, b_{2} \equiv 0$ e $a_{20} \notin \mathbb{Z}$. Pelo Lema 2.16 podemos assumir que $a_{10}=0$. Como antes, a hipótese $a_{20} \notin \mathbb{Z}$ implica que existe 
uma sequência crescente de inteiros positivos $k_{n}>n$, tal que $k_{n} a_{20} \notin \mathbb{Z}$, para todo $n \in \mathbb{N}$. Defina

$$
H(s, t) \doteq \int_{t-s}^{t} b_{1}(\tau) d \tau, \quad 0 \leqslant s, t \leqslant 2 \pi
$$

e

$$
A \doteq H\left(s_{0}, t_{0}\right)=\max _{0 \leqslant s, t \leqslant 2 \pi} H(s, t) .
$$

Também, como antes, podemos assumir que $0<s_{0}, t_{0}, \sigma_{0}<2 \pi$, onde $\sigma_{0}=t_{0}-s_{0}$. Agora considere $\phi \in \mathcal{C}_{c}^{\infty}\left(\left(\sigma_{0}-\delta, \sigma_{0}+\delta\right)\right)$ uma função que satisfaz $0 \leqslant \phi(t) \leqslant 1$ (para todo $t)$ e $\phi(t) \equiv 1$ em uma vizinhança de $\left[\sigma_{0}-\delta / 2, \sigma_{0}+\delta / 2\right]$, onde $\delta>0$ é suficientemente pequeno de modo que $\left(\sigma_{0}-\delta, \sigma_{0}+\delta\right) \subset\left(0, t_{0}\right)$. Para $\xi=\xi(n) \doteq\left(k_{n}, k_{n}, 0, \ldots, 0\right) \in \mathbb{Z}^{N}$, tome $\hat{g}(\xi(n), t)$ como sendo a extensão $2 \pi$-periódica da função

$$
\phi(t) e^{-A k_{n}} e^{i k_{n} a_{20}\left(t_{0}-t\right)}, \quad t \in[0,2 \pi]
$$

Para os demais $\xi \in \mathbb{Z}^{N}$, defina $\hat{g}(\xi, t) \equiv 0$. Como $A k_{n}>A n>0$, a sequência $\hat{g}(\xi, \cdot)$ decai rapidamente, logo define uma função $g \in \mathcal{C}^{\infty}\left(\mathbb{T}^{N+1}\right)$, a qual é dada por

$$
g\left(x_{1}, x_{2}, t\right)=\sum_{n=1}^{\infty} \hat{f}(\xi(n), t) e^{i k_{n}\left(x_{1}+x_{2}\right)}
$$

Procedendo como no caso anterior, verifica-se que $g \in\left(\operatorname{ker}^{t} L\right)^{\circ}$. Mais ainda, se existe $v \in \mathcal{C}^{\infty}\left(\mathbb{T}^{N+1}\right)$ tal que $L v=g$, então os coeficientes parciais de Fourier de $v, \hat{v}(\xi(n), \cdot)$, devem satisfazer

$$
\partial_{t} \hat{v}(\xi(n), t)+i k_{n}\left(a_{20}+i b_{1}(t)\right) \hat{v}(\xi(n), t)=\hat{g}(\xi(n), t),
$$

para todo $t \in \mathbb{T}^{1}$ e para todo $n \in \mathbb{N}$.

Como $a_{20} k_{n} \notin \mathbb{Z}$, do Lema 1.6 segue que

$$
\begin{aligned}
\hat{v}(\xi(n), t) & =\left(1-e^{-2 \pi k_{n}\left(-b_{10}+i a_{20}\right)}\right)^{-1} \int_{0}^{2 \pi} \hat{g}(\xi(n), t-s) \exp \left\{k_{n} \int_{t-s}^{t} b_{1}(\tau) d \tau\right\} e^{-i s k_{n} a_{20}} d s \\
& =\left(1-e^{-2 \pi k_{n}\left(-b_{10}+i a_{20}\right)}\right)^{-1} \int_{0}^{2 \pi} \hat{g}(\xi(n), t-s) e^{k_{n}\left(H(s, t)-i s a_{20}\right)} d s .
\end{aligned}
$$

A definição de $\hat{g}(\xi(n), \cdot)$ implica que 


$$
\begin{aligned}
\left|\hat{v}\left(\xi(n), t_{0}\right)\right| & =\left|1-e^{-2 \pi k_{n}\left(-b_{10}+i a_{20}\right)}\right|^{-1} \int_{s_{0}-\delta}^{s_{0}+\delta} \phi\left(t_{0}-s\right) e^{-k_{n}\left[A-H\left(s, t_{0}\right)\right]} d s \\
& \geqslant \frac{1}{2} \int_{s_{0}-\delta / 2}^{s_{0}+\delta / 2} e^{-k_{n}\left[A-H\left(s, t_{0}\right)\right]} d s .
\end{aligned}
$$

Como no caso anterior, a desigualdade (2.29) gera uma contradição.

O que conclui a demonstração da Proposição 2.18.

Proposição 2.19. Seja L o operador definido em (2.17). Suponha que pelo menos uma das funções $b_{j}$ não se anula identicamente e suponha que $b_{j 0}=0$, para todo $j=1, \ldots, N$. Suponha ainda que $\left(a_{10}, \ldots, a_{N 0}\right) \in \mathbb{Z}^{N}$. Nestas condições, L não é globalmente resolúvel se algum subnivel $\Omega_{r}^{\xi}$ é desconexo, onde

$$
\Omega_{r}^{\xi}=\left\{t \in \mathbb{T}^{1} ; \int_{0}^{t}\langle\xi, \beta(\tau)\rangle d \tau<r\right\}, r \in \mathbb{R} e \xi \in \mathbb{Z}^{N}
$$

Demonstração: A demonstração será por contradição. A ideia central é formada pela seguinte técnica: É um resultado usual (e devido a Hörmander) que a resolubilidade de $L$ implique na validade de uma certa desigualdade envolvendo o operador transposto ${ }^{t} L$ (veja Lema 1.3). Utilizando um subnível desconexo, vamos construir uma sequência de funções que satisfazem tal desigualdade, de modo que, passando ao limite, obtemos uma contradição. Essa técnica comparece nas referências [Ho], [CHo] e [T1].

Como $\left(a_{10}, \ldots, a_{N 0}\right) \in \mathbb{Z}^{N}$, o Lema 2.16 implica que podemos asssumir que o operador $L$ é da forma

$$
L=\frac{\partial}{\partial t}+i \sum_{j=1}^{N} b_{j}(t) \frac{\partial}{\partial x_{j}}
$$

Suponha, por contradição, que $L$ seja globalmente resolúvel. Segue do Lema 1.3 que existem constantes $m \in \mathbb{Z}_{+}$e $C>0$ tais que

$$
\left|\int_{\mathbb{T}^{N+1}} f v\right| \leqslant C \cdot\left(\sum_{|\alpha| \leqslant m} \sup _{\mathbb{T}^{N+1}}\left|\partial^{\alpha} f\right|\right) \cdot\left(\sum_{|\alpha| \leqslant m} \sup _{\mathbb{T}^{N+1}}\left|\partial^{\alpha}\left({ }^{t} L v\right)\right|\right),
$$

quaisquer que sejam $f \in\left(\operatorname{ker}^{t} L\right)^{\circ}$ e $v \in \mathcal{C}^{\infty}\left(\mathbb{T}^{N+1}\right)$.

Por hipótese, existe um subnível $\Omega_{r}^{\xi^{*}}$ que é desconexo. Aplicando o Lema 1.5, existe $r_{0}<r$ tal que o subnível (lembre-se que $\beta(\tau)=\left(b_{1}(\tau), \ldots, b_{N}(\tau)\right)$ ) 


$$
\left\{t \in \mathbb{T}^{1} ; \int_{0}^{t}\left\langle\xi^{*}, \beta(\tau)\right\rangle d \tau<r_{0}\right\}=\left\{t \in \mathbb{T}^{1} ; \sum_{j=1}^{N} \int_{0}^{t} \xi_{j}^{*} b_{j}(\tau) d \tau<r_{0}\right\}
$$

tem duas componentes conexas cujos fechos são disjuntos; mais ainda, existem funções $f_{0}$ e $v_{0}$ em $\mathcal{C}^{\infty}\left(\mathbb{T}^{1}\right)$ com as seguintes propriedades:

$$
\int_{0}^{2 \pi} f_{0}(t) d t=0, \quad \operatorname{supp} f_{0} \cap \Omega_{r_{0}}^{\xi^{*}}=\emptyset, \quad \operatorname{supp} v_{0}^{\prime} \subset \Omega_{r_{0}}^{\xi^{*}}, \quad \text { e } \quad \int_{0}^{2 \pi} f_{0}(t) v_{0}(t) d t>0 .
$$

Para cada $n \in \mathbb{N}$, defina

$$
f_{n}(x, t)=\exp \left\{-n \int_{0}^{t}\left\langle\xi^{*}, \beta(\tau)\right\rangle d \tau\right\} f_{0}(t) e^{-n i\left\langle\xi^{*}, x\right\rangle},(x, t) \in \mathbb{T}^{N} \times \mathbb{T}^{1},
$$

e

$$
v_{n}(x, t)=\exp \left\{n \int_{0}^{t}\left\langle\xi^{*}, \beta(\tau)\right\rangle d \tau\right\} v_{0}(t) e^{n i\left\langle\xi^{*}, x\right\rangle},(x, t) \in \mathbb{T}^{N} \times \mathbb{T}^{1}
$$

Como $b_{j 0}=0$, para todo $j=1, \ldots, N$, segue que $f_{n} \in \mathcal{C}^{\infty}\left(\mathbb{T}^{1}\right)$ e $v_{n} \in \mathcal{C}^{\infty}\left(\mathbb{T}^{1}\right)$, para todo $n \in \mathbb{N}$.

O próximo passo será mostrar que $f_{n} \in\left(\operatorname{ker}^{t} L\right)^{\circ}$. Para tanto, tome $\mu \in \mathcal{D}^{\prime}\left(\mathbb{T}^{N+1}\right)$ tal que ${ }^{t} L \mu=0$. Aplicando série parcial de Fourier com relação às variáveis $\left(x_{1}, \ldots, x_{N}\right)$, obtemos

$$
\partial_{t} \hat{\mu}(\xi, t)-\langle\xi, \beta(t)\rangle \hat{\mu}(\xi, t)=0,
$$

para todo $\xi \in \mathbb{Z}^{N}$ e $t \in \mathbb{T}^{1}$. Assim,

$$
\begin{gathered}
\left\langle\mu, f_{n}\right\rangle=\left\langle\sum_{\xi \in \mathbb{Z}^{N}} \hat{\mu}(\xi, t) \otimes e^{i\langle\xi, x\rangle}, f_{n}(x, t)\right\rangle= \\
\left\langle\hat{\mu}\left(n \xi^{*}, t\right) \otimes e^{i n\left\langle\xi^{*}, x\right\rangle}, \exp \left\{-n \int_{0}^{t}\left\langle\xi^{*}, \beta(\tau)\right\rangle d \tau\right\} f_{0}(t) e^{-i n\left\langle\xi^{*}, x\right\rangle}\right\rangle= \\
(2 \pi)^{N}\left\langle\hat{\mu}\left(n \xi^{*}, t\right), \exp \left\{-n \int_{0}^{t}\left\langle\xi^{*}, \beta(\tau)\right\rangle d \tau\right\} f_{0}(t)\right\rangle= \\
(2 \pi)^{N}\left\langle\exp \left\{-n \int_{0}^{t}\left\langle\xi^{*}, \beta(\tau)\right\rangle d \tau\right\} \hat{\mu}\left(n \xi^{*}, t\right), f_{0}(t)\right\rangle .
\end{gathered}
$$


De (2.31) segue que

$$
\begin{gathered}
\partial_{t}\left(\exp \left\{-n \int_{0}^{t}\left\langle\xi^{*}, \beta(\tau)\right\rangle d \tau\right\} \hat{\mu}\left(n \xi^{*}, t\right)\right)= \\
{\left[\partial_{t} \hat{\mu}\left(n \xi^{*}, t\right)-n\left\langle\xi^{*}, \beta(t)\right\rangle \hat{\mu}\left(n \xi^{*}, t\right)\right] \exp \left\{-n \int_{0}^{t}\left\langle\xi^{*}, \beta(\tau)\right\rangle d \tau\right\}=0 ;}
\end{gathered}
$$

$\log 0$

$$
\exp \left\{-n \int_{0}^{t}\left\langle\xi^{*}, \beta(\tau)\right\rangle d \tau\right\} \hat{\mu}\left(n \xi^{*}, t\right)=c_{n} \in \mathbb{C}
$$

Consequentemente,

$$
\begin{gathered}
\left\langle\mu, f_{n}\right\rangle=(2 \pi)^{N}\left\langle\exp \left\{-n \int_{0}^{t}\left\langle\xi^{*}, \beta(\tau)\right\rangle d \tau\right\} \hat{\mu}\left(n \xi^{*}, t\right), f_{0}(t)\right\rangle= \\
(2 \pi)^{N}\left\langle c_{n}, f_{0}\right\rangle=(2 \pi)^{N} c_{n} \int_{0}^{2 \pi} f_{0}(t) d t=0,
\end{gathered}
$$

qualquer que seja $\mu \in \operatorname{ker}^{t} L$. Portanto, $f_{n} \in\left(\operatorname{ker}^{t} L\right)^{\circ}$, para cada $n \in \mathbb{N}$.

Podemos então aplicar as funções $f_{n}$ e $v_{n}$ na desigualdade (2.30), obtendo

$$
\left|\int_{\mathbb{T}^{N+1}} f_{n} v_{n}\right| \leqslant C \cdot\left(\sum_{|\alpha| \leqslant m} \sup _{\mathbb{T}^{N+1}}\left|\partial^{\alpha} f_{n}\right|\right) \cdot\left(\sum_{|\alpha| \leqslant m} \sup _{\mathbb{T}^{N+1}}\left|\partial^{\alpha}\left({ }^{t} L v_{n}\right)\right|\right),
$$

para todo $n \in \mathbb{N}$.

O lado esquerdo de (2.32) satisfaz

$$
\int_{\mathbb{T}^{N+1}} f_{n} v_{n}=(2 \pi)^{N} \int_{0}^{2 \pi} f_{0}(t) v_{0}(t) d t>0, n \in \mathbb{N} .
$$

Agora vamos fornecer estimativas para o lado direito de (2.32). Utilizando que $\operatorname{supp} f_{0} \cap \Omega_{r_{0}}^{\xi^{*}}=\emptyset$, obtemos

$$
\sum_{|\alpha| \leqslant m} \sup _{\mathbb{T}^{N+1}}\left|\partial^{\alpha} f_{n}\right| \leqslant C_{1} n^{m} \sup _{t \in \mathbb{T}^{1} \backslash \Omega_{r_{0}}^{\xi^{*}}} e^{-n \int_{0}^{t}\left\langle\xi^{*}, \beta(\tau)\right\rangle d \tau} \leqslant C_{1} n^{m} e^{-n r_{0}}
$$

onde $C_{1}>0$ é uma constante que não depende de $n \in \mathbb{N}$. Do mesmo modo, utilizando que $\operatorname{supp} v_{0}^{\prime} \subset \Omega_{r_{0}}^{\xi^{*}}$, obtemos uma constante $C_{2}>0$, a qual não depende de $n \in \mathbb{N}$, tal 
que

$$
\begin{aligned}
\sum_{|\alpha| \leqslant m} \sup _{\mathbb{T}^{N+1}}\left|\partial^{\alpha}\left({ }^{t} L v_{n}\right)\right| & =\sum_{|\alpha| \leqslant m+1} \sup _{\mathbb{T}^{N+1}}\left|\partial^{\alpha}\left[e^{n \int_{0}^{t}\left\langle\xi^{*}, \beta(\tau)\right\rangle d \tau} v_{0}^{\prime}(t) e^{i n\left\langle\xi^{*}, x\right\rangle}\right]\right| \\
& \leqslant C_{2} n^{m} \sup _{t \in \operatorname{supp} v_{0}^{\prime}} e^{n \int_{0}^{t}\left\langle\xi^{*}, \beta(\tau)\right\rangle d \tau} \\
& \leqslant C_{2} n^{m}\left(\sup _{t \in \operatorname{supp} v_{0}^{\prime}} e^{\int_{0}^{t}\left\langle\xi^{*}, \beta(\tau)\right\rangle d \tau}\right)^{n} \\
& =C_{2} n^{m} e^{n \int_{0}^{t_{1}}\left\langle\xi^{*}, \beta(\tau)\right\rangle d \tau}
\end{aligned}
$$

onde $t_{1} \in \operatorname{supp} v_{0}^{\prime} \subset \Omega_{r_{0}}^{\xi^{*}}$ é um ponto de máximo da restrição de $e^{\int_{0}^{t}\left\langle\xi^{*}, \beta(\tau)\right\rangle d \tau}$ ao conjunto $\operatorname{supp} v_{0}^{\prime} \subset \Omega_{r_{0}}^{\xi^{*}}$.

Por fim, defina $c^{*} \doteq \int_{0}^{t_{1}}\left\langle\xi^{*}, \beta(\tau)\right\rangle d \tau-r_{0}<0$, a qual é uma constante que não depende de $n \in \mathbb{N}$. De $(2.34)$ e (2.35) segue que

$$
\left(\sum_{|\alpha| \leqslant m} \sup _{\mathbb{T}^{N+1}}\left|\partial^{\alpha} f_{n}\right|\right) \cdot\left(\sum_{|\alpha| \leqslant m} \sup _{\mathbb{T}^{N+1}} \mid \partial^{\alpha}\left({ }^{t} L v_{n}\right)\right) \leqslant C_{1} C_{2} n^{2 m} e^{n c^{*}} .
$$

Aplicando (2.32), (2.33) e (2.36) obtemos

$$
0<(2 \pi)^{N} \int_{0}^{2 \pi} f_{0}(t) v_{0}(t) d t \leqslant C_{1} C_{2} n^{2 m} e^{n c^{*}}, \text { para todo } n \in \mathbb{N}
$$

O que é uma contradição, uma vez que $\lim _{n \rightarrow \infty} C_{1} C_{2} n^{2 m} e^{n c^{*}}=0$.

Portanto, $L$ não é globalmente resolúvel.

Agora mostraremos que as condições impostas sobre os coeficientes, no caso (II) do Teorema 2.6, são suficientes para a resolubilidade global.

Proposição 2.20. Para um operador L do tipo (2.17), suponha que pelo menos um coeficiente $b_{j}$ não seja identicamente nulo e que $b_{j 0}=0$, para todo $j=1, \ldots, N$. Além disso, se $\left(a_{10}, \ldots, a_{N 0}\right) \in \mathbb{Z}^{N}$ e se todos os subníveis $\Omega_{r}^{\xi}$ são conexos, então L é globalmente resolúvel.

Demonstração: A ideia central da demonstração é mostrar que $\left(\operatorname{ker}^{t} L\right)^{\circ}=L \mathcal{C}^{\infty}\left(\mathbb{T}^{N+1}\right)$. Basta mostrar que, para cada $f \in\left(\operatorname{ker}^{t} L\right)^{\circ}$, existe uma função $u \in \mathcal{C}^{\infty}\left(\mathbb{T}^{N+1}\right)$ que satisfaz a equação $L u=f$. 
Aplicando o Lema (2.16), podemos assumir que o operador $L$ é do tipo

$$
L=\frac{\partial}{\partial t}+i \sum_{j=1}^{N} b_{j}(t) \frac{\partial}{\partial x_{j}}
$$

Utilizando série parcial de Fourier nas variáveis $\left(x_{1}, \ldots, x_{N}\right)$, escrevemos

$$
f(x, t)=\sum_{\xi \in \mathbb{Z}^{N}} \hat{f}(\xi, t) e^{i\langle\xi, x\rangle}
$$

e

$$
u(x, t)=\sum_{\xi \in \mathbb{Z}^{N}} \hat{u}(\xi, t) e^{i\langle\xi, x\rangle} .
$$

Desta forma, a equação diferencial parcial $L u=f$ nos leva à seguinte sequência de equações diferenciais ordinárias

$$
\partial_{t} \hat{u}(\xi, t)-\langle\xi, \beta(t)\rangle \hat{u}(\xi, t)=\hat{f}(\xi, t), t \in \mathbb{T}^{1}, \xi \in \mathbb{Z}^{N}
$$

onde lembramos que $\beta(t)=\left(b_{1}(t), \ldots, b_{N}(t)\right)$.

Como $b_{j 0}=0$, para todo $j=1, \ldots, N$, obtemos $\int_{0}^{2 \pi}\langle\xi, \beta(\tau)\rangle d \tau=0$, para cada $\xi \in \mathbb{Z}^{N}$. Mais ainda,

$$
\begin{gathered}
-{ }^{t} L\left(\exp \left\{-\int_{0}^{t}\langle\xi, \beta(\tau)\rangle d \tau\right\} e^{-i\langle\xi, x\rangle}\right)= \\
\partial_{t}\left(\exp \left\{-\int_{0}^{t}\langle\xi, \beta(\tau)\rangle d \tau\right\}\right) e^{-i\langle\xi, x\rangle}+\sum_{j=1}^{N} i b_{j}(t) \exp \left\{-\int_{0}^{t}\langle\xi, \beta(\tau)\rangle d \tau\right\} \frac{\partial}{\partial x_{j}}\left(e^{-i\langle\xi, x\rangle}\right)= \\
-\langle\xi, \beta(t)\rangle \exp \left\{-\int_{0}^{t}\langle\xi, \beta(\tau)\rangle d \tau\right\} e^{-i\langle\xi, x\rangle}+ \\
\left(\sum_{j=1}^{N} \xi_{j} b_{j}(t)\right) \exp \left\{-\int_{0}^{t}\langle\xi, \beta(\tau)\rangle d \tau\right\} e^{-i\langle\xi, x\rangle}=0 ;
\end{gathered}
$$

o que implica que a função

$$
\phi_{\xi}(x, t) \doteq \exp \left\{-\int_{0}^{t}\langle\xi, \beta(\tau)\rangle d \tau\right\} e^{-i\langle\xi, x\rangle}
$$


pertence a $\operatorname{ker}^{t} L$, para cada $\xi \in \mathbb{Z}^{N}$. Como $f \in\left(\operatorname{ker}^{t} L\right)^{\circ}$, obtemos

$$
\begin{aligned}
0 & =\left\langle\exp \left\{-\int_{0}^{t}\langle\xi, \beta(\tau)\rangle d \tau\right\} e^{-i\langle\xi, x\rangle}, f\right\rangle=(2 \pi)^{N}\left\langle\exp \left\{-\int_{0}^{t}\langle\xi, \beta(\tau)\rangle d \tau\right\}, \hat{f}(\xi, t)\right\rangle \\
& =(2 \pi)^{N} \int_{0}^{2 \pi} \exp \left\{-\int_{0}^{t}\langle\xi, \beta(\tau)\rangle d \tau\right\} \hat{f}(\xi, t) d t
\end{aligned}
$$

Assim, do Lema 1.6 segue que cada equação (2.37) possui infinitas soluções em $\mathcal{C}^{\infty}\left(\mathbb{T}^{1}\right)$, as quais possuem a forma geral

$$
\hat{u}(\xi, t)=e^{\int_{0}^{t}\langle\xi, \beta(\tau)\rangle d \tau}\left(\int_{t_{\xi}}^{t} e^{-\int_{0}^{s}\langle\xi, \beta(\tau)\rangle d \tau} \hat{f}(\xi, s) d s+C_{\xi}\right), t \in \mathbb{T}^{1}
$$

onde $t_{\xi} \in \mathbb{T}^{1}$ e $C_{\xi} \in \mathbb{C}$.

O próximo passo será fixar uma determinada solução de (2.37), de modo que a sequência formada por tais soluções seja rapidamente decrescente.

Para cada $\xi \in \mathbb{Z}^{N}$, fixe $t_{\xi}$ um ponto de $\mathbb{T}^{1}$ tal que

$$
\int_{0}^{t_{\xi}}\langle\xi, \beta(\tau)\rangle d \tau=\sup _{t \in \mathbb{T}^{1}}\left\{\int_{0}^{t}\langle\xi, \beta(\tau)\rangle d \tau\right\}
$$

ou, equivalentemente, tal que

$$
-\int_{0}^{t_{\xi}}\langle\xi, \beta(\tau)\rangle d \tau=\inf _{t \in \mathbb{T}^{1}}\left\{-\int_{0}^{t}\langle\xi, \beta(\tau)\rangle d \tau\right\} .
$$

Fixe $\hat{u}(\xi, \cdot)$ a solução de $(2.37)$ em $\mathcal{C}^{\infty}\left(\mathbb{T}^{1}\right)$ que é dada por

$$
\hat{u}(\xi, t) \doteq \int_{t_{\xi}}^{t} \exp \left\{\int_{0}^{t}\langle\xi, \beta(\tau)\rangle d \tau-\int_{0}^{s}\langle\xi, \beta(\tau)\rangle d \tau\right\} \hat{f}(\xi, s) d s, t \in \mathbb{T}^{1}
$$

Para concluir esta demonstração, falta apenas mostrar que $(\hat{u}(\xi, \cdot))$ decai rapidamente, ou seja, dados $m \in \mathbb{Z}_{+}$e $n \in \mathbb{Z}_{+}$, precisamos encontrar $C(m, n)>0$ tal que

$$
|\xi|^{m}\left|\partial_{t}^{n} \hat{u}(\xi, t)\right| \leqslant C(m, n)
$$

para todo $t \in \mathbb{T}^{1}$ e $\xi \in \mathbb{Z}^{N}$. Para tanto, será necessário reescrever cada solução $\hat{u}(\xi, \cdot)$.

Para $t \in \mathbb{T}^{1}$, considere

$$
r_{t} \doteq-\int_{0}^{t}\langle\xi, \beta(\tau)\rangle d \tau
$$


Note que $t$ e $t_{\xi}$ pertencem ao conjunto

$$
\tilde{\Omega}_{t} \doteq\left\{s \in \mathbb{T}^{1} ;-\int_{0}^{s}\langle\xi, \beta(\tau)\rangle d \tau \leqslant r_{t}\right\}
$$

o qual é conexo pelo Lema (1.4). Logo existe um caminho $\gamma_{t} \subset \tilde{\Omega}_{t}$, o qual é um arco de circunferência, ligando $t_{\xi}$ a $t$. Mais ainda, como $\gamma_{t} \subset \tilde{\Omega}_{t}$, temos

$$
-\int_{0}^{s}\langle\xi, \beta(\tau)\rangle d \tau \leqslant r_{t}=-\int_{0}^{t}\langle\xi, \beta(\tau)\rangle d \tau, \text { para todo } s \in \gamma_{t} .
$$

Como, para cada $t \in \mathbb{T}^{1}$, existem apenas dois arcos de circunferência ligando $t_{\xi}$ a $t$, cuja reunião é $\mathbb{T}^{1}$, e como

$$
\begin{aligned}
& \int_{0}^{2 \pi} \exp \left\{\int_{0}^{2 \pi}\langle\xi, \beta(\tau)\rangle d \tau-\int_{0}^{s}\langle\xi, \beta(\tau)\rangle d s\right\} \hat{f}(\xi, s) d s= \\
& \int_{0}^{2 \pi} \exp \left\{-\int_{0}^{s}\langle\xi, \beta(\tau)\rangle d \tau\right\} \hat{f}(\xi, s) d s=0, \text { por }(2.39)
\end{aligned}
$$

podemos escolher $\gamma_{t}$ de modo que

$$
\hat{u}(\xi, t)=\int_{\gamma_{t}} \exp \left\{\int_{0}^{t}\langle\xi, \beta(\tau)\rangle d \tau-\int_{0}^{s}\langle\xi, \beta(\tau)\rangle d \tau\right\} \hat{f}(\xi, s) d s .
$$

A identidade (2.44) e a estimativa (2.43) implicam que

$$
|\hat{u}(\xi, t)| \leqslant 2 \pi\|\hat{f}(\xi, \cdot)\|_{\infty}
$$

onde $t \in \mathbb{T}^{1}$ foi fixado de maneira arbitrária, logo

$$
\|\hat{u}(\xi, \cdot)\|_{\infty} \leqslant 2 \pi\|\hat{f}(\xi, \cdot)\|_{\infty}
$$

Daí, o decaimento rápido de $\hat{f}(\xi, \cdot)$ implica que

$$
|\xi|^{m}\|\hat{u}(\xi, \cdot)\|_{\infty} \leqslant C(m, 0)
$$

para todo $\xi \in \mathbb{Z}^{N}$; logo vale (2.42) para qualquer $m \in \mathbb{Z}_{+}$e para $n=0$. Utilizando um processo recursivo envolvendo (2.37) e procedendo de modo análogo ao que foi feito 
acima, obtemos a validade de (2.42) para quaisquer $m \in \mathbb{Z}_{+}$e $n \in \mathbb{Z}_{+}$. O que conclui a demonstração da Proposição 2.20.

\section{III Final da demonstração do Teorema 2.6: caso (III)}

Para concluir a demonstração do Teorema 2.6, consideramos agora o caso (III), onde estamos sob a hipótese de que $b_{j 0} \neq 0$, para algum $j \in\{1, \ldots, N\}$.

Mostremos que (III.1) é uma condição necessária para a resolubilidade global de $L$.

Proposição 2.21. Seja L o operador dado por (2.17) e suponha que $b_{j 0} \neq 0$, para algum indice $j=1, \ldots, N$. Suponha também que não vale a condição (III.1) no Teorema 2.6, ou seja, $\operatorname{dim} \operatorname{span}\left\{b_{1}, \ldots, b_{N}\right\} \geqslant 2$. Neste caso, L não é globalmente resolúvel.

Demonstração: Tome $m \in\{1, \ldots, N\}$ tal que $b_{m 0} \neq 0$. Como $\operatorname{dim} \operatorname{span}\left\{b_{1}, \ldots, b_{N}\right\} \geqslant$ 2 , existe $\ell \neq m$ tal que $b_{\ell}$ e $b_{m}$ são $\mathbb{R}$ - linearmente independentes. Reordenando as variáveis $x_{1}, \ldots, x_{N}$, podemos assumir que $m=1$ e $\ell=2$. Do Lema 2.2 segue que existem inteiros não nulos $p$ e $q, p \neq q$, tais que $\theta(t) \doteq p b_{1}(t)+q b_{2}(t)$ muda de sinal e $\theta_{0} \doteq \frac{1}{2 \pi} \int_{0}^{2 \pi} \theta(t) d t=p b_{10}+q b_{20}<0$.

Utilizando série parcial de Fourier, vamos construir uma função $f$ em $\left(\operatorname{ker}^{t} L\right)^{\circ} \backslash$ $L \mathcal{C}^{\infty}\left(\mathbb{T}^{N+1}\right)$, a qual será da forma

$$
f\left(x_{1}, x_{2}, t\right)=\sum_{n=1}^{\infty} \hat{f}(\xi(n), t) e^{i\langle\xi(n), x\rangle},
$$

onde $\xi(n)=(n p, n q, 0, \ldots, 0) \in \mathbb{Z}^{N}$, para todo $n \in \mathbb{N}$.

Iniciaremos agora a construção dos coeficientes $\hat{f}(\xi(n), \cdot) \in \mathcal{C}^{\infty}\left(\mathbb{T}^{1}\right)$. Defina

$$
H(s, t)=\int_{t-s}^{t} \theta(\tau) d \tau, \quad s, t \in[0,2 \pi]
$$

$\mathrm{e}$

$$
A=H\left(s_{0}, t_{0}\right)=\max _{0 \leqslant s, t \leqslant 2 \pi} H(s, t)
$$

Note que $A>0$ pois $\theta$ muda de sinal. Como na demonstração da Proposição 2.18, podemos supor que $0<s_{0}, t_{0}, \sigma_{0}<2 \pi$, onde $\sigma_{0}=t_{0}-s_{0}$. Tome $\phi \in \mathcal{C}_{c}^{\infty}\left(\left(\sigma_{0}-\delta, \sigma_{0}+\delta\right)\right)$, uma função tal que $0 \leqslant \phi(t) \leqslant 1$ (para todo $t$ ) e $\phi(t) \equiv 1$ em uma vizinhança de 
$\left[\sigma_{0}-\delta / 2, \sigma_{0}+\delta / 2\right]$, onde $\delta>0$ é suficientemente pequeno de modo que $\left(\sigma_{0}-\delta, \sigma_{0}+\delta\right) \subset$ $\left(0, t_{0}\right)$.

Temos todos os ingredientes para definir os coeficientes $\hat{f}(\xi(n), \cdot)$. Para cada $n \in \mathbb{N}$, defina $\hat{f}(\xi(n), \cdot)$ pela extensão $2 \pi$ - periódica da função

$$
e^{-n A} \phi(t) e^{i n\left(p a_{10}+q a_{20}\right)\left(t_{0}-t\right)}, \quad t \in[0,2 \pi]
$$

O termo $e^{-n A}$ implica que $\hat{f}(\xi(n), \cdot)$ decai rapidamente, uma vez que $A>0$. Assim, $\hat{f}(\xi(n), \cdot)$ define uma função $f$ em $\mathcal{C}^{\infty}\left(\mathbb{T}^{N+1}\right)$, dada pela fórmula $(2.45)$.

Afirmamos que $f \in\left(\operatorname{ker}^{t} L\right)^{\circ}$. De fato, seja $\mu \in \mathcal{D}^{\prime}\left(\mathbb{T}^{N+1}\right)$ tal que ${ }^{t} L \mu=0$. Escrevendo $\mu$ por meio da sua série parcial de Fourier com relação às variáveis $\left(x_{1}, \ldots, x_{N}\right)$, obtemos

$$
\partial_{t} \hat{\mu}(\xi, \cdot)+i\left\langle\xi, \alpha_{0}+i \beta(t)\right\rangle \hat{\mu}(\xi, \cdot)=0
$$

onde lembramos que $\alpha_{0}=\left(a_{10}, \ldots, a_{N 0}\right)$ e $\beta(t)=\left(b_{1}(t), \ldots, b_{N}(t)\right)$. Em particular, para os índices $\xi(n)=(n p, n q, 0, \ldots, 0)$ definidos acima, obtemos

$$
\partial_{t} \hat{\mu}(-\xi(n), \cdot)+n\left[\theta(\cdot)-i\left(p a_{10}+q a_{20}\right)\right] \hat{\mu}(-\xi(n), \cdot)=0 .
$$

O Lema 1.6 implica que $\hat{\mu}(-\xi(n), \cdot)=0$, uma vez que $\theta_{0} \neq 0$. Assim,

$$
\langle\mu, f\rangle=(2 \pi)^{N} \sum_{n=1}^{\infty}\langle\hat{\mu}(-\xi(n), \cdot), \hat{f}(\xi(n), \cdot)\rangle=0
$$

qualquer que seja $\mu \in \operatorname{ker}^{t} L, \operatorname{logo} f \in\left(\operatorname{ker}^{t} L\right)^{\circ}$.

Para concluir a demonstração, resta apenas mostrar que $f \notin L \mathcal{C}^{\infty}\left(\mathbb{T}^{N+1}\right)$. Suponha, por contradição, que existe $u \in \mathcal{C}^{\infty}\left(\mathbb{T}^{N+1}\right)$, solução da equação $L u=f$. Sendo assim, os coeficientes parciais de Fourier $\hat{u}(\xi(n), \cdot)$ e $\hat{f}(\xi(n), \cdot)$ das funções $u$ e $f$, respectivamente, satisfazem

$$
\begin{aligned}
& \partial_{t} \hat{u}(\xi(n), t)+i\left\langle\xi(n), \alpha_{0}+i \beta(t)\right\rangle \hat{u}(\xi(n), t)= \\
& \partial_{t} \hat{u}(\xi(n), t)+n\left[-\theta(t)+i\left(p a_{10}+q a_{20}\right)\right] \hat{u}(\xi(n), t)=\hat{f}(\xi(n), t), \quad t \in \mathbb{T}^{1}, \quad n \in \mathbb{N} .
\end{aligned}
$$


Como $\theta_{0}<0$, segue do Lema 1.6 que a equação (2.46) possui uma única solução, a qual pode ser escrita na forma

$$
\hat{u}(\xi(n), t)=\left(1-e^{2 \pi n\left(\theta_{0}-i\left(p a_{10}+q a_{20}\right)\right)}\right)^{-1} \int_{0}^{2 \pi} \hat{f}(\xi(n), t-s) e^{-i s n\left(p a_{10}+q a_{20}\right)} e^{n \int_{t-s}^{t} \theta(\tau) d \tau} d s
$$

A definição de $\hat{f}(\xi(n), \cdot)$ implica que, para todo $t \in\left[t_{0}, 2 \pi\right]$, temos

$$
\hat{u}(\xi(n), t)=\left(1-e^{2 \pi n\left(\theta_{0}-i\left(p a_{10}+q a_{20}\right)\right)}\right)^{-1} \int_{t-\sigma_{0}-\delta}^{t-\sigma_{0}+\delta} \phi(t-s) e^{-i n\left(t_{0}-t\right)\left(p a_{10}+q a_{20}\right)} e^{-n(A-H(s, t))} d s
$$

e calculando em $t=t_{0}$ obtemos

$$
\hat{u}\left(\xi(n), t_{0}\right)=\left(1-e^{2 \pi n\left(\theta_{0}-i\left(p a_{10}+q a_{20}\right)\right)}\right)^{-1} \int_{s_{0}-\delta}^{s_{0}+\delta} \phi\left(t_{0}-s\right) e^{-n\left(A-H\left(s, t_{0}\right)\right)} d s .
$$

Daí, $\phi \geqslant 0$ e $\phi \equiv 1$ em $\left[\sigma_{0}-\delta / 2, \sigma_{0}+\delta / 2\right]$ implicam que

$$
\begin{aligned}
\left|\hat{u}\left(\xi(n), t_{0}\right)\right| & \geqslant\left|1-e^{2 \pi n\left(\theta_{0}-i\left(p a_{10}+q a_{20}\right)\right)}\right|^{-1} \int_{s_{0}-\delta / 2}^{s_{0}+\delta / 2} e^{-n\left(A-H\left(s, t_{0}\right)\right)} d s \\
& \geqslant \frac{1}{2} \int_{s_{0}-\delta / 2}^{s_{0}+\delta / 2} e^{-n\left(A-H\left(s, t_{0}\right)\right)} d s
\end{aligned}
$$

Por fim, a função $A-H\left(s, t_{0}\right)$ tem um zero em $s_{0}$ e $A-H\left(s, t_{0}\right) \geqslant 0$, o que implica que $s_{0}$ não é de ordem ímpar. Procedendo como na parte final da demonstração da Proposição 2.18, obtemos uma constante $M>0$ tal que

$$
\left|\hat{u}\left(\xi(n), t_{0}\right)\right| \geqslant M n^{-1 / 2}, \quad \text { para todo } n \in \mathbb{N}
$$

o que é uma contradição, pois $\hat{u}\left(\xi(n), t_{0}\right)$ decai rapidamente.

O que conclui a demonstração da Proposição 2.21.

O próximo passo será mostrar que a condição (III.2) também é necessária para a resolubilidade global de $L$.

Proposição 2.22. Para o operador $L$ dado por (2.17), suponha que $b_{j 0} \neq 0$ para algum indice $j=1, \ldots, N$. Suponha também que a condição (III.2) no Teorema 2.6 falha, ou seja, $b_{j}$ muda de sinal, para algum índice $j$. Sob tais condições, o operador L não é globalmente resolúvel. 
Demonstração: Essa demonstração segue a mesma linha da demonstração da Proposição 2.21.

Suponha que $b_{m 0} \neq 0$ e que $b_{\ell}$ muda de sinal. Afirmamos que existem inteiros não nulos $p$ e $q$ tais que $p b_{m}(t)+q b_{\ell}(t)$ muda de sinal e $p b_{m 0}+q b_{\ell 0}<0$. De fato, se $b_{m}$ e $b_{\ell}$ são $\mathbb{R}$ - linearmente independentes, então tal afirmação segue do Lema 2.2. Por outro lado, se $b_{m}$ e $b_{\ell}$ são $\mathbb{R}$ - linearmente dependentes, então $b_{\ell}(t)=r b_{m}(t), t \in \mathbb{T}^{1}, r \in \mathbb{R} \backslash\{0\}$. Assim, $b_{m}$ e $p b_{m}+b_{\ell}=(p+r) b_{m}$ também mudam de sinal; tomando $p \in \mathbb{Z} \backslash\{0\}$ tal que $(p+r) b_{m 0}<0$, obtemos a veracidade da afirmação (note que tomamos $q=1$ ).

Portanto, repetindo a demonstração da Proposição 2.21 segue que $L$ não é globalmente resolúvel.

A próxima proposição concluirá a demonstração de que as condições (III.1)-(III.3) são necessárias para a resolubilidade global de L. Para tanto, precisamos provar que a condição (III.3) é necessária. Como nas proposições 2.18 e 2.21, utilizaremos série parcial de Fourier para construir uma função em $\left(\operatorname{ker}^{t} L\right)^{\circ} \backslash L \mathcal{C}^{\infty}\left(\mathbb{T}^{N+1}\right)$. Para provar que não existe $u \in \mathcal{C}^{\infty}\left(\mathbb{T}^{N+1}\right)$ solução de $L u=f$, vamos empregar o Lema 1.6 em cada equação envolvendo os coeficientes parciais de Fourier $\hat{u}(\xi, \cdot)$ e $\hat{f}(\xi, \cdot)$, de modo que $(1.8)$ fornecerá uma expressão para $\hat{u}(\xi, \cdot)$. Será importante controlar o coeficiente que aparece em (1.8); diante de tal objetivo, precisaremos do seguinte resultado:

Lema 2.23. Dado um par de vetores $\alpha=\left(\alpha_{1}, \ldots, \alpha_{N}\right)$ e $\beta=\left(\beta_{1}, \ldots, \beta_{N}\right)$ em $\mathbb{R}^{N}$, a condição Diofantina (DC2) é equivalente à seguinte condição:

(DC3) existem constantes $C>0$ e $\gamma>0$ tais que

$$
\left|1-e^{2 \pi\langle\xi, \beta-i \alpha\rangle}\right| \geqslant C|\xi|^{-\gamma}
$$

para todo $\xi \in \mathbb{Z}^{N}$ tal que $\langle\xi, \beta-i \alpha\rangle \notin i \mathbb{Z}$.

Demonstração: Mostremos que (DC3) implica (DC2). Suponha que o par $(\alpha, \beta)$ não satisfaz (DC2). Então existe uma sequência $\left(\xi(n), \tau_{n}\right)_{n \in \mathbb{N}} \subset \mathbb{Z}^{N} \times \mathbb{Z}$ tal que $\tau_{n}+\langle\xi(n), \alpha+$ $i \beta\rangle \neq 0,|\xi(n)|+\left|\tau_{n}\right| \geqslant n \mathrm{e}$

$$
\left|\tau_{n}+\langle\xi(n), \alpha+i \beta\rangle\right|<\left(|\xi(n)|+\left|\tau_{n}\right|\right)^{-n}
$$


para todo $n \in \mathbb{N}$. Em particular, $\left|\tau_{n}+\langle\xi(n), \alpha\rangle\right| \rightarrow 0$, quando $n \rightarrow \infty$. Além disso, a estimativa

$$
\left|\tau_{n}+\langle\xi(n), \alpha\rangle\right| \geqslant\left|\tau_{n}\right|-|\langle\xi(n), \alpha\rangle| \geqslant\left|\tau_{n}\right|-|\xi(n)| \max _{j=1, \ldots, N}\left|\alpha_{j}\right|
$$

implica que a sequência $|\xi(n)|$ não pode ser limitada.

Substituindo $\left(\xi(n), \tau_{n}\right)$ por $-\left(\xi(n), \tau_{n}\right)$ se necessário, podemos supor que $\langle\xi(n), \beta\rangle \leqslant$ 0 , para todo $n \in \mathbb{N}$. A menos de tomarmos uma subsequência, podemos supor que $\langle\xi(n), \beta\rangle<0$ (para todo $n \in \mathbb{N}$ ) ou que $\langle\xi(n), \beta\rangle=0$ (para todo $n \in \mathbb{N}$ ).

Assuma primeiro que $\langle\xi(n), \beta\rangle<0$, para todo $n \in \mathbb{N}$. Deste modo, temos

$$
\begin{gathered}
\left|1-e^{2 \pi\langle\xi(n), \beta-i \alpha\rangle}\right| \leqslant\left|1-e^{2 \pi\langle\xi(n), \beta\rangle} \cos (2 \pi\langle\xi(n), \alpha\rangle)\right|+e^{2 \pi\langle\xi(n), \beta\rangle}|\operatorname{sen}(2 \pi\langle\xi(n), \alpha\rangle)| \leqslant \\
|1-\cos (2 \pi\langle\xi(n), \alpha\rangle)|+\left|1-e^{2 \pi\langle\xi(n), \beta\rangle}\right||\cos (2 \pi\langle\xi(n), \alpha\rangle)|+e^{2 \pi\langle\xi(n), \beta\rangle}|\operatorname{sen}(2 \pi\langle\xi(n), \alpha\rangle)| \leqslant \\
|1-\cos (2 \pi\langle\xi(n), \alpha\rangle)|+\left|1-e^{2 \pi\langle\xi(n), \beta\rangle}\right|+|\operatorname{sen}(2 \pi\langle\xi(n), \alpha\rangle)|= \\
\left|1-\cos \left(2 \pi\left[\tau_{n}+\langle\xi(n), \alpha\rangle\right]\right)\right|+\left|1-e^{2 \pi\langle\xi(n), \beta\rangle}\right|+\left|\operatorname{sen}\left(2 \pi\left[\tau_{n}+\langle\xi(n), \alpha\rangle\right]\right)\right| \leqslant \\
4 \pi\left|\tau_{n}+\langle\xi(n), \alpha\rangle\right|+2 \pi|\langle\xi(n), \beta\rangle| \leqslant 6 \pi\left|\tau_{n}+\langle\xi(n), \alpha\rangle+i\langle\xi(n), \beta\rangle\right|= \\
6 \pi\left|\tau_{n}+\langle\xi(n), \alpha+i \beta\rangle\right|<6 \pi\left(|\xi(n)|+\left|\tau_{n}\right|\right)^{-n}<6 \pi|\xi(n)|^{-n}
\end{gathered}
$$

para todo $n \in \mathbb{N}$. Como $|\xi(n)|$ não é limitada e $\langle\xi(n), \beta-i \alpha\rangle \notin i \mathbb{Z}$ (uma vez que $\langle\xi(n), \beta\rangle<0)$, segue que o par $(\alpha, \beta)$ não satisfaz a condição (DC3).

Agora assuma que $\langle\xi(n), \beta\rangle=0$, para todo $n \in \mathbb{N}$. Como $\tau_{n}+\langle\xi(n), \alpha\rangle=\tau_{n}+$ $\langle\xi(n), \alpha+i \beta\rangle \neq 0$ e $\left|\tau_{n}+\langle\xi(n), \alpha\rangle\right|<n^{-n}$, para todo $n \in \mathbb{N}$, segue que $\langle\xi(n), \alpha\rangle$ não é um inteiro. Mais ainda,

$$
\begin{gathered}
\left|1-e^{2 \pi\langle\xi(n), \beta-i \alpha\rangle}\right|=\left|1-e^{-2 \pi i\langle\xi(n), \alpha\rangle}\right|=\left|1-e^{-2 \pi i\left(\tau_{n}+\langle\xi(n), \alpha\rangle\right)}\right| \leqslant 2 \pi\left|\tau_{n}+\langle\xi(n), \alpha\rangle\right|= \\
2 \pi\left|\tau_{n}+\langle\xi(n), \alpha+i \beta\rangle\right|<2 \pi\left(|\xi(n)|+\left|\tau_{n}\right|\right)^{-n}<2 \pi|\xi(n)|^{-n},
\end{gathered}
$$

o que implica que o par $(\alpha, \beta)$ não satisfaz (DC3), uma vez que $|\xi(n)|$ não é limitada e $\langle\xi(n), \beta-i \alpha\rangle=-i\langle\xi(n), \alpha\rangle \notin i \mathbb{Z}$.

Reciprocamente, mostremos que (DC2) implica (DC3). Suponha que o par $(\alpha, \beta)$ não satisfaz (DC3). Então existe uma sequência $(\xi(n))_{n \in \mathbb{N}} \subset \mathbb{Z}^{N}$ tal que $|\xi(n)| \geqslant n$, 
$\langle\xi(n), \beta-i \alpha\rangle \notin i \mathbb{Z} \mathrm{e}$

$$
\left|1-e^{2 \pi\langle\xi(n), \beta-i \alpha\rangle}\right|<|\xi(n)|^{-n}
$$

para todo $n \in \mathbb{N}$. Em particular, obtemos $|\langle\xi(n), \beta\rangle| \rightarrow 0$, quando $n \rightarrow \infty$, $\operatorname{logo}$

$$
\left|1-e^{2 \pi\langle\xi(n), \beta\rangle}\right| \geqslant e^{-1} 2 \pi|\langle\xi(n), \beta\rangle|,
$$

para todo $n \in \mathbb{N}$ suficientemente grande.

De (2.49) também segue que existe uma sequência de números inteiros $\left(\tau_{n}\right)$ tal que $\tau_{n}+\langle\xi(n), \alpha\rangle \rightarrow 0$, quando $n \rightarrow \infty$. De fato, basta tomar, para cada $n \in \mathbb{N}, t_{n}=$ $-\llbracket\langle\xi(n), \alpha\rangle \rrbracket$ ou $\tau_{n}=-\rrbracket\langle\xi(n), \alpha\rangle \llbracket$, onde $\llbracket\langle\xi(n), \alpha\rangle \rrbracket$ denota o maior inteiro menor ou igual a $\langle\xi(n), \alpha\rangle$ e $\rrbracket\langle\xi(n), \alpha\rangle \llbracket$ denota o menor inteiro maior ou igual a $\langle\xi(n), \alpha\rangle$. Assim,

$$
\left|\operatorname{sen}\left(2 \pi\left[\tau_{n}+\langle\xi(n), \alpha\rangle\right]\right)\right| \geqslant \frac{1}{2}\left|\tau_{n}+\langle\xi(n), \alpha\rangle\right| 2 \pi,
$$

para todo $n \in \mathbb{N}$ suficientemente grande.

Consequentemente, de (2.49) e (2.50) segue que, para $n$ suficientemente grande, vale

$$
e^{-1} 2 \pi|\langle\xi(n), \beta\rangle| \leqslant\left|1-e^{2 \pi\langle\xi(n), \beta\rangle}\right| \leqslant\left|1-e^{2 \pi\langle\xi(n), \beta-i \alpha\rangle}\right|<|\xi(n)|^{-n}
$$

enquanto que (2.49) e (2.51) implicam

$$
\begin{gathered}
\pi\left|\tau_{n}+\langle\xi(n), \alpha\rangle\right| \leqslant\left|\operatorname{sen}\left(2 \pi\left[\tau_{n}+\langle\xi(n), \alpha\rangle\right]\right)\right| \leqslant 2 e^{2 \pi\langle\xi(n), \beta\rangle}\left|\operatorname{sen}\left(2 \pi\left[\tau_{n}+\langle\xi(n), \alpha\rangle\right]\right)\right| \leqslant \\
2\left|1-e^{2 \pi\langle\xi(n), \beta-i \alpha\rangle}\right|<2|\xi(n)|^{-n},
\end{gathered}
$$

para todo $n$ suficientemente grande.

Assim,

$$
\begin{aligned}
\left|\tau_{n}+\langle\xi(n), \alpha+i \beta\rangle\right| & \leqslant\left|\tau_{n}+\langle\xi(n), \alpha\rangle\right|+|\langle\xi(n), \beta\rangle| \\
& <|\xi(n)|^{-n} \max \left\{\frac{e}{2 \pi}, \frac{2}{\pi}\right\}=\frac{2}{\pi}|\xi(n)|^{-n}
\end{aligned}
$$

para todo $n \in \mathbb{N}$ suficientemente grande. O que implicará que (DC2) não pode valer. De fato, se $(\alpha, \beta)$ satisfaz (DC2), temos

$$
C\left(\left|\tau_{n}\right|+|\xi(n)|\right)^{-\gamma} \leqslant\left|\tau_{n}+\langle\xi(n), \alpha+i \beta\rangle\right|<\frac{2}{\pi}|\xi(n)|^{-n}
$$


$\log 0$

$$
0<\frac{C \pi}{2}<|\xi(n)|^{-n+\gamma}\left(1+\frac{\left|\tau_{n}\right|}{|\xi(n)|}\right)^{\gamma}
$$

e como $0 \leqslant\left|\tau_{n}+\langle\xi(n), \alpha\rangle\right|<1$, obtemos $\left|\tau_{n}\right| \leqslant 1+|\xi(n)| \max _{j=1 \ldots, N}\left|\alpha_{j}\right| ;$ consequentemente,

$$
0<\frac{C \pi}{2}<|\xi(n)|^{-n+\gamma}\left(1+\frac{1}{|\xi(n)|}+\max _{j=1 \ldots, N}\left|\alpha_{j}\right|\right)^{\gamma}
$$

para todo $n$ suficientemente grande. O que é uma contradição, pois

$$
\lim _{n \rightarrow \infty}|\xi(n)|^{-n+\gamma}\left(1+\frac{1}{|\xi(n)|}+\max _{j=1 \ldots, N}\left|\alpha_{j}\right|\right)^{\gamma}=0
$$

Portanto, (DC2) implica (DC3).

Mostremos que (III.3) também é necessária. As proposições 2.21 e 2.22 nos permitem supor que estão satisfeitas as condições (III.1) e (III.2), logo podemos assumir que existe uma função que não muda de sinal $b \in \mathcal{C}^{\infty}\left(\mathbb{T}^{1}, \mathbb{R}\right) \backslash\{0\}$ e que existe $\left(\lambda_{1}, \ldots, \lambda_{N}\right) \in$ $\mathbb{R}^{N} \backslash\{0\}$ tal que $b_{j}(t)=\lambda_{j} b(t), t \in \mathbb{T}^{1}$, para todo $j=1, \ldots, N$. Assim, podemos escrever o operador $L$ (dado em (2.17)) da seguinte forma

$$
L=\frac{\partial}{\partial t}+\sum_{j=1}^{N}\left(a_{j 0}+i \lambda_{j} b(t)\right) \frac{\partial}{\partial x_{j}} .
$$

Note que, $b_{0}=\frac{1}{2 \pi} \int_{0}^{2 \pi} b(t) d t \neq 0$, pois $b \not \equiv 0$ e $b$ não muda de sinal.

Proposição 2.24. Seja $L$ o operador (2.52), onde $\lambda=\left(\lambda_{1}, \ldots, \lambda_{N}\right) \in \mathbb{R}^{N} \backslash\{0\}$ e $b \in \mathcal{C}^{\infty}\left(\mathbb{T}^{1}, \mathbb{R}\right)$ é uma função que não se anula identicamente e não muda de sinal. Suponha que seja falsa a condição (III.3) no Teorema 2.6, ou seja, o par $\left(\alpha_{0}, b_{0} \lambda\right)$ não satisfaz (DC2), onde $\alpha_{0}=\left(a_{10}, \ldots, a_{N 0}\right)$ e $b_{0}=(2 \pi)^{-1} \int_{0}^{2 \pi} b(t) d t$. Nestas condições, o operador L não é globalmente resolúvel.

Demonstração: Vamos construir uma função $f \in\left(\operatorname{ker}^{t} L\right)^{\circ}$ de modo que a equação $L u=f$ não tenha solução em $\mathcal{C}^{\infty}\left(\mathbb{T}^{N+1}\right)$. Utilizaremos série parcial de Fourier nas variáveis $\left(x_{1}, \ldots, x_{N}\right)$ para construir $f$ da forma

$$
f(x, t)=\sum_{n=1}^{\infty} \hat{f}(\xi(n), t) e^{i\langle\xi(n), x\rangle}
$$


onde $\xi(n)$ é uma determinada sequência em $\mathbb{Z}^{N}$, a qual passamos a descrever:

Do Lema 2.23 segue que o par $\left(\alpha_{0}, b_{0} \lambda\right)$ não satisfaz a conição (DC3), logo existe uma sequência $(\xi(n))_{n \in \mathbb{N}} \subset \mathbb{Z}^{N}$ tal que $|\xi(n+1)|>|\xi(n)| \geqslant n,\left\langle\xi(n), b_{0} \lambda-i \alpha_{0}\right\rangle \notin i \mathbb{Z}$ e

$$
\left|1-\exp \left\{2 \pi\left\langle\xi(n), b_{0} \lambda-i \alpha_{0}\right\rangle\right\}\right|<|\xi(n)|^{-n}
$$

para todo $n \in \mathbb{N}$. Tomando uma subsequência e substituindo $\xi(n)$ por $-\xi(n)$, se necessário, podemos supor que ocorre uma das seguintes situações:

(i) $\left\langle\xi(n), b_{0} \lambda\right\rangle>0 \mathrm{e}$

$$
\left|1-\exp \left\{2 \pi\left\langle\xi(n), b_{0} \lambda-i \alpha_{0}\right\rangle\right\}\right|<|\xi(n)|^{-n}, \text { para todo } n \in \mathbb{N},
$$

$\mathrm{ou}$

(ii) $\left\langle\xi(n), b_{0} \lambda\right\rangle=0,\left\langle\xi(n), \alpha_{0}\right\rangle \notin \mathbb{Z} \mathrm{e}$

$$
\left|1-\exp \left\{-2 \pi i\left\langle\xi(n), \alpha_{0}\right\rangle\right\}\right|<|\xi(n)|^{-n}, \text { para todo } n \in \mathbb{N} \text {. }
$$

Desta forma, a demonstração naturalmente se divide em duas etapas.

Suponha primeiro que ocorre (i).

Passemos a construir as funções $\hat{f}(\xi(n), \cdot) \in \mathcal{C}^{\infty}\left(\mathbb{T}^{1}\right)$. Seja $\delta>0$ suficientemente pequeno de modo que $\left(\frac{\pi}{2}-\delta, \frac{\pi}{2}+\delta\right) \subset(0, \pi)$ e tome $\phi$ uma função em $\mathcal{C}_{c}^{\infty}\left(\left(\frac{\pi}{2}-\delta, \frac{\pi}{2}+\delta\right)\right)$, satisfazendo $0 \leqslant \phi(t) \leqslant 1$ (para todo $t$ ) e $\phi \equiv 1$ em uma vizinhança de $\left[\frac{\pi-\delta}{2}, \frac{\pi+\delta}{2}\right]$.

Defina $\hat{f}(\xi(n), \cdot)$ pela extensão $2 \pi$ - periódica de

$$
|\xi(n)|^{-n / 2} \phi(t) e^{i(\pi-t)\left\langle\xi(n), \alpha_{0}\right\rangle}, \quad t \in[0,2 \pi] .
$$

O termo $|\xi(n)|^{-n / 2}$ implica que $\hat{f}(\xi(n), \cdot)$ decai rapidamente, logo define uma função $f \in \mathcal{C}^{\infty}\left(\mathbb{T}^{N+1}\right)$, dada pela fórmula (2.53). Afirmamos que tal $f$ pertence a $\left(\operatorname{ker}^{t} L\right)^{\circ}$. De fato, se $\mu \in \mathcal{D}^{\prime}\left(\mathbb{T}^{N+1}\right)$ e ${ }^{t} L \mu=-L \mu=0$, então os coeficientes parciais de Fourier de $\mu$ satisfazem

$$
\partial_{t} \hat{\mu}(\xi, \cdot)+i\left\langle\xi, \alpha_{0}+i b(\cdot) \lambda\right\rangle \hat{\mu}(\xi, \cdot)=0,
$$


para todo $\xi \in \mathbb{Z}^{N}$. Em particular,

$$
\partial_{t} \hat{\mu}(-\xi(n), \cdot)-i\left\langle\xi(n), \alpha_{0}+i b(\cdot) \lambda\right\rangle \hat{\mu}(-\xi(n), \cdot)=0,
$$

para todo $n \in \mathbb{N}$. Como $\left\langle\xi(n), b_{0} \lambda\right\rangle \neq 0$, do Lema 1.6 segue que $\hat{\mu}(-\xi(n), \cdot)=0$. Daí,

$$
\langle\mu, f\rangle=(2 \pi)^{N} \sum_{n=1}^{\infty}\langle\hat{\mu}(-\xi(n), \cdot), \hat{f}(\xi(n), \cdot)\rangle=0
$$

Assim, $\langle\mu, f\rangle=0$ para toda $\mu \in \operatorname{ker}^{t} L ; \operatorname{logo} f \in\left(\operatorname{ker}^{t} L\right)^{\circ}$.

Por fim, mostremos que não existe $u \in \mathcal{C}^{\infty}\left(\mathbb{T}^{N+1}\right)$ tal que $L u=f$. Suponha por contradição que exista uma tal $u$. Novamente a série parcial de Fourier nos leva às seguintes equações

$$
\partial_{t} \hat{u}(\xi(n), t)+i\left\langle\xi(n), \alpha_{0}+i b(t) \lambda\right\rangle \hat{u}(\xi(n), t)=\hat{f}(\xi(n), t), \quad t \in \mathbb{T}^{1},
$$

para todo $n \in \mathbb{N}$. Como $\left\langle\xi(n), b_{0} \lambda\right\rangle>0$, a equação (2.57) possui uma única solução (Lema 1.6), a qual escrevemos por

$$
\hat{u}(\xi(n), t)=\left(1-e^{2 \pi c_{n}}\right)^{-1} \int_{0}^{2 \pi} \hat{f}(\xi(n), t-s) e^{\langle\xi(n), \lambda\rangle \int_{t-s}^{t} b(\tau) d \tau} e^{-i s\left\langle\xi(n), \alpha_{0}\right\rangle} d s,
$$

onde definimos $c_{n} \doteq\left\langle\xi(n), b_{0} \lambda-i \alpha_{0}\right\rangle$ para simplificar a notação.

A definição de $\hat{f}(\xi(n), \cdot)$ implica que, para $t \in[\pi, 2 \pi]$, temos

$$
\hat{u}(\xi(n), t)=\left(1-e^{2 \pi c_{n}}\right)^{-1} \int_{t-(\pi / 2+\delta)}^{t-(\pi / 2-\delta)}|\xi(n)|^{-n / 2} \phi(t-s) e^{i(\pi-t)\left\langle\xi(n), \alpha_{0}\right\rangle} e^{\langle\xi(n), \lambda\rangle \int_{t-s}^{t} b(\tau) d \tau} d s
$$

Em particular, para $t=\pi$ obtemos

$$
\hat{u}(\xi(n), \pi)=\left(1-e^{2 \pi c_{n}}\right)^{-1}|\xi(n)|^{-n / 2} \int_{\pi / 2-\delta}^{\pi / 2+\delta} \phi(\pi-s) e^{\langle\xi(n), \lambda\rangle \int_{\pi-s}^{\pi} b(\tau) d \tau} d s
$$

mais ainda, como $b$ não muda de sinal e como $\langle\xi(n), \lambda\rangle b_{0}>0$, obtemos $\langle\xi(n), \lambda\rangle b(t) \geqslant 0$, o que implica

$$
|\hat{u}(\xi(n), \pi)| \geqslant\left|1-e^{2 \pi c_{n}}\right|^{-1}|\xi(n)|^{-n / 2} \delta
$$


De (2.55) segue que

$$
|\hat{u}(\xi(n), \pi)| \geqslant|\xi(n)|^{n / 2} \delta \geqslant n^{n / 2} \delta, \quad \text { para todo } n \in \mathbb{N}
$$

o que é uma contradição, pois $\lim _{n \rightarrow \infty} \hat{u}(\xi(n), \pi)=0$, já que $\hat{u}(\xi(n), \pi)$ decai rapidamente.

Portanto, $L$ não é globalmente resolúvel quando ocorre (i).

Para concluirmos a demonstração, suponha que ocorre (ii). A ideia é aplicar os mesmos argumentos utilizados na situação (i) para exibir uma $f$ em $\left(\operatorname{ker}^{t} L\right)^{\circ} \backslash L \mathcal{C}^{\infty}\left(\mathbb{T}^{N+1}\right)$. As funções $\hat{f}(\xi(n), \cdot)$ têm a mesma forma que no caso (i), ou seja, elas são a extensão $2 \pi$ - periódica das funções

$$
|\xi(n)|^{-n / 2} \phi(t) e^{i(\pi-t)\left\langle\xi(n), \alpha_{0}\right\rangle}, t \in[0,2 \pi] .
$$

Note que agora temos $\langle\xi(n), \lambda\rangle b_{0}=0$ e como $b_{0} \neq 0$, obtemos $\langle\xi(n), \lambda\rangle=0$, para todo $n \in \mathbb{N}$, mas $\left\langle\xi(n), \alpha_{0}\right\rangle \notin \mathbb{Z}$, o que implica (como em (i)) que $\hat{\mu}(-\xi(n), \cdot)=0$, para toda $\mu \in \operatorname{ker}^{t} L$. Daí, $f$ dada por $(2.53)$ pertence a $\left(\operatorname{ker}^{t} L\right)^{\circ}$.

A equação (2.57) agora torna-se

$$
\partial_{t} \hat{u}(\xi(n), t)+i\left\langle\xi(n), \alpha_{0}\right\rangle \hat{u}(\xi(n), t)=\hat{f}(\xi(n), t), \quad t \in \mathbb{T}^{1}
$$

para cada $n \in \mathbb{N}$. A qual possui única solução (Lema 1.6) que pode ser escrita por

$$
\hat{u}(\xi(n), t)=\left(1-e^{-2 \pi i\left\langle\xi(n), \alpha_{0}\right\rangle}\right)^{-1} \int_{0}^{2 \pi} \hat{f}(\xi(n), t-s) e^{-i s\left\langle\xi(n), \alpha_{0}\right\rangle} d s
$$

Por fim, a definição de $\hat{f}(\xi(n), \cdot)$ implica que, para $t \in[\pi, 2 \pi]$, temos

$$
\hat{u}(\xi(n), t)=\left(1-e^{-2 \pi i\left\langle\xi(n), \alpha_{0}\right\rangle}\right)^{-1}|\xi(n)|^{-n / 2} \int_{t-(\pi / 2+\delta)}^{t-(\pi / 2-\delta)} \phi(t-s) e^{i(\pi-t)\left\langle\xi(n), \alpha_{0}\right\rangle} d s
$$

e calculando em $t=\pi$ obtemos

$$
\hat{u}(\xi(n), \pi)=\left(1-e^{-2 \pi i\left\langle\xi(n), \alpha_{0}\right\rangle}\right)^{-1}|\xi(n)|^{-n / 2} \int_{\pi / 2-\delta}^{\pi / 2+\delta} \phi(t-s) d s .
$$

Daí, $\phi \geqslant 0$ e $\phi \equiv 1$ em $[(\pi-\delta) / 2,(\pi+\delta) / 2]$ implicam que

$$
|\hat{u}(\xi(n), \pi)| \geqslant\left|1-e^{-2 \pi i\left\langle\xi(n), \alpha_{0}\right\rangle}\right|^{-1}|\xi(n)|^{-n / 2} \delta
$$


e a estimativa (2.56) implica que

$$
|\hat{u}(\xi(n), \pi)| \geqslant|\xi(n)|^{n / 2} \delta \geqslant n^{n / 2} \delta, \quad \text { para todo } n \in \mathbb{N}
$$

o que, como antes, é uma contradição, pois $\hat{u}(\xi(n), \pi)$ decai rapidamente.

Portanto, $L$ também não é globalmente resolúvel quando ocorre (ii). O que conclui a demonstração da Proposição 2.24.

Para finalizar a demonstração do Teorema 2.6, mostremos que as condições (III.1)(III.3) implicam a resolubilidade global de $L$.

Proposição 2.25. Considere o operador $L$ dado por (2.17). Suponha que $b_{j 0} \neq 0$, para algum $j=1, \ldots, N$ e suponha que valem as seguintes condições:

(III.1) $\operatorname{dim} \operatorname{span}\left\{b_{1}, \ldots, b_{N}\right\}=1$.

(III.2) cada função $b_{j}$ não muda de sinal, $j=1, \ldots, N$.

(III.3) o par $\left(\alpha_{0}, \beta_{0}\right)$ satisfaz (DC2).

Nestas condições, L é globalmente resolúvel.

Demonstração: Seja $f \in\left(\operatorname{ker}^{t} L\right)^{\circ}$. Vamos utilizar série parcial de Fourier para exibir uma solução de $L u=f$ em $\mathcal{C}^{\infty}\left(\mathbb{T}^{N+1}\right)$. Antes de iniciarmos a busca por $u$, observe que as hipóteses implicam que existe $b \in \mathcal{C}^{\infty}\left(\mathbb{T}^{1}, \mathbb{R}\right) \backslash\{0\}$, uma função que não muda de sinal, e existe $\lambda=\left(\lambda_{1}, \ldots, \lambda_{N}\right) \in \mathbb{R}^{N} \backslash\{0\}$, tais que $\beta(t)=\left(b_{1}(t), \ldots, b_{N}(t)\right)=b(t) \lambda$, para todo $t \in \mathbb{T}^{1}$. Nestas condições, podemos escrever

$$
L=\frac{\partial}{\partial t}+\sum_{j=1}^{N}\left(a_{j 0}+i \lambda_{j} b(t)\right) \frac{\partial}{\partial x_{j}} .
$$

Defina $b_{0}=\frac{1}{2 \pi} \int_{0}^{2 \pi} b(t) d t$. Como $b(t)$ não é identicamente nula e não muda de sinal, temos $b_{0} \neq 0$.

Escrevendo $u$ e $f$ por meio das suas séries parciais de Fourier com relação às variáveis $\left(x_{1}, \ldots, x_{N}\right)$, temos

$$
u(x, t)=\sum_{\xi \in \mathbb{Z}^{N}} \hat{u}(\xi, t) e^{i\langle\xi, x\rangle}
$$


e

$$
f(x, t)=\sum_{\xi \in \mathbb{Z}^{N}} \hat{f}(\xi, t) e^{i\langle\xi, x\rangle}
$$

Desta forma, encontrar $u \in \mathcal{C}^{\infty}\left(\mathbb{T}^{N+1}\right)$ tal que $L u=f$ é o mesmo que encontrar uma sequência de funções $\hat{u}(\xi, \cdot) \in \mathcal{C}^{\infty}\left(\mathbb{T}^{1}\right)$, que seja rapidamente decrescente e satisfaça

$$
\partial_{t} \hat{u}(\xi, t)+i\left\langle\xi, \alpha_{0}+i b(t) \lambda\right\rangle \hat{u}(\xi, t)=\hat{f}(\xi, t), \quad \text { para todo } t \in \mathbb{T}^{1} \text { e } \xi \in \mathbb{Z}^{N},
$$

onde $\alpha_{0}=\left(a_{10}, \ldots, a_{N 0}\right)$.

Do Lema 1.6 seguirá que, dependendo do índice $\xi \in \mathbb{Z}^{N}$, a equação (2.60) possuirá infinitas soluções ou uma única solução. Logo, a demonstração naturalmente se divide em três etapas.

(i) $\xi \in \mathbb{Z}^{N}$ é tal que $\left\langle\xi, b_{0} \lambda\right\rangle=0$ e $\left\langle\xi, \alpha_{0}\right\rangle \in \mathbb{Z}$. Para tais índices, como $b_{0} \neq 0$, obtemos $\langle\xi, \lambda\rangle=0$. Daí a equação (2.60) se reduz a

$$
\partial_{t} \hat{u}(\xi, t)+i\left\langle\xi, \alpha_{0}\right\rangle \hat{u}(\xi, t)=\hat{f}(\xi, t), \quad \text { para todo } t \in \mathbb{T}^{1}
$$

Além disso, $\left\langle\xi, \alpha_{0}\right\rangle \in \mathbb{Z}$ implica que $\phi_{\xi}(x, t) \doteq e^{i t\left\langle\xi, \alpha_{0}\right\rangle} e^{-i\langle\xi, x\rangle}$ é uma função de $\mathcal{C}^{\infty}\left(\mathbb{T}^{N+1}\right)$. Um cálculo direto mostra que ${ }^{t} L \phi_{\xi}=0 ; \operatorname{logo}$

$$
0=\left\langle\phi_{\xi}, f\right\rangle=(2 \pi)^{N} \int_{0}^{2 \pi} \hat{f}(\xi, t) e^{i t\left\langle\xi, \alpha_{0}\right\rangle} d t
$$

Assim, a equação (2.61) possui infinitas soluções. Fixemos a solução

$$
\hat{u}(\xi, t)=\int_{0}^{t} \hat{f}(\xi, s) e^{i(s-t)\left\langle\xi, \alpha_{0}\right\rangle} d s, t \in \mathbb{T}^{1}
$$

(ii) $\xi \in \mathbb{Z}^{N}$ satisfaz $\left\langle\xi, b_{0} \lambda\right\rangle \neq 0$. Neste caso, a equação (2.60) possui uma única solução, a qual pode ser escrita de maneiras distintas. Para tornar a notação mais curta, definimos

$$
c_{\xi}=\left\langle\xi, \beta_{0}-i \alpha_{0}\right\rangle=\left\langle\xi, b_{0} \lambda-i \alpha_{0}\right\rangle
$$

Quando $\left\langle\xi, b_{0} \lambda\right\rangle<0$, escrevemos a solução por

$$
\hat{u}(\xi, t)=\left(1-e^{2 \pi c_{\xi}}\right)^{-1} \int_{0}^{2 \pi} \hat{f}(\xi, t-s) e^{\langle\xi, \lambda\rangle \int_{t-s}^{t} b(\tau) d \tau} e^{-i s\left\langle\xi, \alpha_{0}\right\rangle} d s, t \in \mathbb{T}^{1}
$$


Por outro lado, quando $\left\langle\xi, b_{0} \lambda\right\rangle>0$, escrevemos a solução por

$$
\hat{u}(\xi, t)=\left(e^{-2 \pi c_{\xi}}-1\right)^{-1} \int_{0}^{2 \pi} \hat{f}(\xi, t+s) e^{-\langle\xi, \lambda\rangle \int_{t}^{t+s} b(\tau) d \tau} e^{i s\left\langle\xi, \alpha_{0}\right\rangle} d s, t \in \mathbb{T}^{1}
$$

(iii) $\xi \in \mathbb{Z}^{N}$ é tal que $\left\langle\xi, b_{0} \lambda\right\rangle=0$ e $\left\langle\xi, \alpha_{0}\right\rangle \notin \mathbb{Z}$. Novamente, a equação (2.60) se reduz a (2.61) (como no caso (i)) e tal equação possui uma única solução, a qual escrevemos por

$$
\hat{u}(\xi, t)=\left(1-e^{-2 \pi i\left\langle\xi, \alpha_{0}\right\rangle}\right)^{-1} \int_{0}^{2 \pi} \hat{f}(\xi, t-s) e^{-i s\left\langle\xi, \alpha_{0}\right\rangle} d s, t \in \mathbb{T}^{1} .
$$

Em resumo, até aqui definimos uma sequência $\hat{u}(\xi, \cdot)_{\xi \in \mathbb{Z}^{N}}$ de funções em $\mathcal{C}^{\infty}\left(\mathbb{T}^{1}\right)$, as quais são soluções de (2.60).

Para finalizar a demonstração, basta mostrar que tal sequência é rapidamente decrescente. Nessa parte, utilizaremos a validade da condição Diofantina (DC2) para o par $\left(\alpha_{0}, b_{0} \lambda\right)$, a qual implica na validade de (DC3) (ver Lema 2.23). Logo existem $C>0$ e $\gamma>0$ tais que

$$
\left|1-e^{2 \pi c_{\xi}}\right|=\left|1-e^{2 \pi\left\langle\xi, b_{0} \lambda-i \alpha_{0}\right\rangle}\right| \geqslant C|\xi|^{-\gamma}
$$

para todo $\xi \in \mathbb{Z}^{N}$ tal que $\left\langle\xi, b_{0} \lambda-i \alpha_{0}\right\rangle \notin i \mathbb{Z}$.

Vamos mostrar que, dados $m \in \mathbb{Z}_{+}$e $n \in \mathbb{Z}_{+}$, existe $C(m, n)>0$ tal que

$$
|\xi|^{m}\left|\partial_{t}^{n} \hat{u}(\xi, t)\right| \leqslant C(m, n)
$$

para todo $t \in \mathbb{T}^{1}$ e $\xi \in \mathbb{Z}^{N}$.

Considere primeiro o caso em que $n=0$. Note que a solução dada por (2.62) satisfaz

$$
|\hat{u}(\xi, t)| \leqslant \int_{0}^{t}|\hat{f}(\xi, s)| d s, t \in \mathbb{T}^{1}
$$

Do mesmo modo, a solução dada por (2.63) satisfaz

$$
|\hat{u}(\xi, t)| \leqslant\left|1-e^{2 \pi c \xi}\right|^{-1} \int_{0}^{2 \pi}|\hat{f}(\xi, t-s)| e^{\langle\xi, \lambda\rangle \int_{t-s}^{t} b(\tau) d \tau} d s
$$


mais ainda, de $(2.66)$ e do fato de que $\langle\xi, \lambda\rangle \int_{t-s}^{t} b(\tau) d \tau \leqslant 0$ (já que $\left\langle\xi, b_{0} \lambda\right\rangle<0$ e $b$ não muda de sinal) obtemos

$$
|\hat{u}(\xi, t)| \leqslant C^{-1}|\xi|^{\gamma} \int_{0}^{2 \pi}|\hat{f}(\xi, t-s)| d s .
$$

Analogamente, (2.66) implica que a solução dada por (2.65) também satisfaz (2.69).

Com relação à solução dada por (2.64), note que de (2.66) obtemos $\left|e^{-2 \pi c_{\xi}}-1\right|=$ $\left|1-e^{2 \pi c_{-\xi}}\right| \geqslant C|-\xi|^{-\gamma}$. Logo tal solução satisfaz

$$
|\hat{u}(\xi, t)| \leqslant C^{-1}|\xi|^{\gamma} \int_{0}^{2 \pi}|\hat{f}(\xi, t+s)| e^{-\langle\xi, \lambda\rangle \int_{t}^{t+s} b(\tau) d \tau} d s
$$

mais ainda, como $\langle\xi, \lambda\rangle \int_{t}^{t+s} b(\tau) d \tau \geqslant 0$ (já que $\left\langle\xi, b_{0} \lambda\right\rangle>0$ e $b$ não muda de sinal) obtemos

$$
|\hat{u}(\xi, t)| \leqslant C^{-1}|\xi|^{\gamma} \int_{0}^{2 \pi}|\hat{f}(\xi, t+s)| d s
$$

As estimativas (2.68), (2.69) e (2.70), mais o decaimento rápido de $\hat{f}(\xi, \cdot)$ implicam que vale (2.67) para $n=0$ e para todo $m \in \mathbb{Z}_{+}$.

Considere agora o caso em que $n=1$. Lembre-se que a equação (2.60) implica que

$$
\begin{aligned}
\left|\partial_{t} \hat{u}(\xi, t)\right| & \leqslant\left|\left\langle\xi, \alpha_{0}+i b(t) \lambda\right\rangle \| \hat{u}(\xi, t)\right|+|\hat{f}(\xi, t)| \\
& \leqslant|\xi|\left(\left\|\alpha_{0}\right\|+\|b\|_{\infty}\|\lambda\|\right)|\hat{u}(\xi, t)|+|\hat{f}(\xi, t)| .
\end{aligned}
$$

Daí, do caso $n=0$ e do decaimento rápido de $\hat{f}(\xi, \cdot)$ segue que vale $(2.67)$ para $n=1$ e para todo $m \in \mathbb{Z}_{+}$.

Por fim, utilizando indução e aplicando esse processo recursivo obtemos a validade de (2.67) para todo $n \in \mathbb{Z}_{+}$e $m \in \mathbb{Z}_{+}$. Portanto, $\hat{u}(\xi, \cdot)$ decai rapidamente e, assim, define a função

$$
u(x, t)=\sum_{\xi \in \mathbb{Z}^{N}} \hat{u}(\xi, t) e^{i\langle\xi, x\rangle},
$$

a qual satisfaz $L u=f$. O que finaliza a demonstração da Proposição 2.25

Concluímos assim o estudo da resolubilidade gobal de $L$ (dado por (2.1)), o que nos permite determinar quando que a imagem de $L$ é ou não fechada. 
O próximo passo é investigar quando que a imagem de $L$ tem codimensão finita. Já sabemos que tal propriedade não ocorre quando $L$ não é globalmente resolúvel; por outro lado, quando $L$ é globalmente resolúvel, basta analisar a dimensão de $\operatorname{ker}^{t} L$ (ver Lema 1.1). Para tanto, vamos analisar as demonstrações das proposições 2.17 (suficiência), 2.20 e 2.25 .

Na demonstração da Proposição 2.17 (suficiência), vimos que as funções $\phi_{(\xi, \tau)}(x, t)=$ $e^{i(\langle\xi, x\rangle+\tau t)}(\operatorname{ver}(2.21))$ estão em $\operatorname{ker}^{t} L$, desde que $\tau+\left\langle\xi, \alpha_{0}\right\rangle=0$. Seja $\mathcal{A} \doteq\{(\xi, \tau) \in$ $\left.\mathbb{Z}^{N} \times \mathbb{Z} ; \tau+\left\langle\xi, \alpha_{0}\right\rangle=0\right\}$. Escrevendo uma distribuição $\mu \in \operatorname{ker}^{t} L$ em série de Fourier,

$$
\mu=\sum_{(\xi, \tau) \in \mathbb{Z}^{N} \times \mathbb{Z}} \hat{\mu}(\xi, \tau) e^{i(\langle\xi, x\rangle+\tau t)}
$$

da identidade ${ }^{t} L \mu=0$ obtemos $\left(\tau+\left\langle\xi, \alpha_{0}\right\rangle\right) \hat{\mu}(\xi, \tau)=0$, para todo $(\xi, \tau) \in \mathbb{Z}^{N} \times \mathbb{Z}$; assim, $\hat{\mu}(\xi, \tau)=0$ se $(\xi, \tau) \notin \mathcal{A}$, ou seja

$$
\mu=\sum_{(\xi, \tau) \in \mathcal{A}} \hat{\mu}(\xi, \tau) e^{i(\langle\xi, x\rangle+\tau t)} .
$$

Portanto, $\operatorname{dim} \operatorname{ker}^{t} L<\infty$ se, e somente se $\mathcal{A}$ é finito; o que ocorre se, e somente se $\mathcal{A}=\{(0,0)\}$. Em particular, $L$ (dado por (2.18)) é fortemente resolúvel se, e somente se, $\left(\alpha_{0}, 0\right)$ satisfaz (DC1) (ver página 23). Portanto, $L$ dado por (2.1), nas condições do item (I) no Teorema 2.6, é fortemente resolúvel se, e somente se, $\left(\alpha_{0}, 0\right)$ satisfaz (DC1).

Olhemos agora para a demonstração da Proposição 2.20. Nas condições de tal proposição, vimos que, para cada $\xi \in \mathbb{Z}^{N}$, as funções $\phi_{\xi}(x, t)=\exp \left\{-\int_{0}^{t}\langle\xi, \beta(\tau)\rangle d \tau\right\} e^{-i\langle\xi, x\rangle}$ (ver (2.38)) estão em $\operatorname{ker}^{t} L$; como $\left\{\phi_{\xi} ; \xi \in \mathbb{Z}^{N}\right\}$ é um subconjunto linearmente independente de $\mathcal{D}^{\prime}\left(\mathbb{T}^{N+1}\right)$, segue que $\operatorname{dim} \operatorname{ker}^{t} L=\infty$, logo $L$ (dado por (2.1)) não é fortemente resolúvel nas condições do item (II) do Teorema 2.6.

Por fim, analisemos a demonstração da Proposição 2.25. No item (i) dessa demonstração vimos que, para cada $\xi \in \mathbb{Z}^{N}$ tal que $\left\langle\xi, b_{0} \lambda\right\rangle=0$ e $\left\langle\xi, \alpha_{0}\right\rangle \in \mathbb{Z}$, a função $\phi_{\xi}(x, t)=$ $e^{i t\left\langle\xi, \alpha_{0}\right\rangle} e^{-i\langle\xi, x\rangle}$ está em $\operatorname{ker}^{t} L$. Assim, se $\mathcal{B} \doteq\left\{\xi \in \mathbb{Z}^{N} ;\left\langle\xi, b_{0} \lambda\right\rangle=0\right.$ e $\left.\left\langle\xi, \alpha_{0}\right\rangle \in \mathbb{Z}\right\}$ é infinito, obtemos $\operatorname{dim} \operatorname{ker}^{t} L=\infty$. Por outro lado, veremos que $\mathcal{B}$ ser finito implica que $\operatorname{dim} \operatorname{ker}^{t} L<\infty$. De fato, se $\mu \in \operatorname{ker}^{t} L$, então escrevendo $\mu$ em série parcial de Fourier 
nas variáveis $\left(x_{1}, \ldots, x_{N}\right)$,

$$
\mu=\sum_{\xi \in \mathbb{Z}^{N}} \hat{\mu}(\xi, \cdot) \otimes e^{i\langle\xi, x\rangle}
$$

obtemos

$$
\partial_{t} \hat{\mu}(\xi, \cdot)+i\left\langle\xi, \alpha_{0}+i b(\cdot) \lambda\right\rangle \hat{\mu}(\xi, \cdot)=0, \text { para todo } \xi \in \mathbb{Z}^{N}
$$

Do Lema 1.6 segue que $\hat{\mu}(\xi, \cdot)=0$ se $\xi \notin \mathcal{B}$, logo

$$
\mu=\sum_{\xi \in \mathcal{B}} \hat{\mu}(\xi, \cdot) \otimes e^{i\langle\xi, x\rangle}
$$

Deste modo, $\mathcal{B}$ finito implica que $\operatorname{dim} \operatorname{ker}^{t} L<\infty$. Note que $\mathcal{B}$ finito significa que $\mathcal{B}=\{0\}$ e, assim, (DC2) é o mesmo que (DC1). Portanto, nas hipóteses da Proposição 2.25, $L$ é fortemente resolúvel se, e somente se, $\left(\alpha_{0}, \beta_{0}\right)=\left(\alpha_{0}, b_{0} \lambda\right)$ satisfaz (DC1).

Resumiremos os comentários acima com a seguinte observação, a qual caracteriza a resolubilidade forte de $L$.

Observação 2.26. O operador $L$ dado por (2.1) é fortemente resolúvel se, e somente se:

- $b_{j} \equiv 0$ para todo $j=1, \ldots, N$ e $\left(\alpha_{0}, 0\right)$ satisfaz (DC1);

- $b_{j 0} \neq 0$ para algum $j \in\{1, \ldots, N\}$, dim $\operatorname{span}\left\{b_{1}, \ldots, b_{N}\right\}=1$, cada função $b_{j}$ não muda de sinal e $\left(\alpha_{0}, \beta_{0}\right)$ satisfaz (DC1). 



$\frac{\sqrt{3}}{3}$

\section{Hipoeliticidade global para campos vetoriais do tipo tubo}

Um campo vetorial $L: \mathcal{D}^{\prime}\left(\mathbb{T}^{n}\right) \rightarrow \mathcal{D}^{\prime}\left(\mathbb{T}^{n}\right)$ (no sentido do Capítulo 1) é dito globalmente hipoelítico quando as condições $\mu \in \mathcal{D}^{\prime}\left(\mathbb{T}^{n}\right)$ e $L \mu \in \mathcal{C}^{\infty}\left(\mathbb{T}^{n}\right)$ implicam que $\mu \in \mathcal{C}^{\infty}\left(\mathbb{T}^{n}\right)$.

O objetivo do presente capítulo é estabelecer a hipoeliticidade global para os operadores do tipo tubo, os quais tiveram a resolubilidade global determinada pelo Teorema 2.6 no Capítulo 2. Lembremos que tais operadores são do tipo

$$
L=\frac{\partial}{\partial t}+\sum_{j=1}^{N}\left(a_{j}(t)+i b_{j}(t)\right) \frac{\partial}{\partial x_{j}} \quad\left(\mathrm{em} \mathbb{T}^{N+1}\right)
$$

onde $a_{j}$ e $b_{j}$ são funções em $\mathcal{C}^{\infty}\left(\mathbb{T}^{1} ; \mathbb{R}\right)$, para cada $j=1, \ldots, N$. Denotamos por $(x, t)$ as coordenadas em $\mathbb{T}^{N} \times \mathbb{T}^{1}$, onde $x=\left(x_{1}, \ldots, x_{N}\right) \in \mathbb{T}^{N}$.

Como antes, forneceremos condições sobre os coeficientes de $L$ que irão caracterizar a hipoeliticidade global, as quais são similares às que aparecem na caracterização da resolubilidade global de $L$. Vale adiantar que a condição envolvendo a conexidade dos subníveis $\Omega_{r}^{\xi}$, no item (II) do Teorema 2.6, não desempenha papel na hipoeliticidade, isto é, $L$ não será globalmente hipoelítico na situação (II) do Teorema 2.6, que é quando $L$ não satisfaz a condição $(\mathcal{P})$.

Antes de enunciarmos tal caracterização, lembremos que $a_{j 0}=(2 \pi)^{-1} \int_{0}^{2 \pi} a_{j}(t) d t$, $b_{j 0}=(2 \pi)^{-1} \int_{0}^{2 \pi} b_{j}(t) d t, \alpha_{0}=\left(a_{10}, \ldots, a_{N 0}\right)$ e $\beta_{0}=\left(b_{10}, \ldots, b_{N 0}\right)$. Lembremos também a seguinte condição Diofantina para um par $(\alpha, \beta) \in \mathbb{R}^{N} \times \mathbb{R}^{N}$ : 
(DC1) existem constantes $C>0$ e $\gamma>0$ tais que

$$
|\tau+\langle\xi, \alpha+i \beta\rangle| \geqslant C(|\xi|+|\tau|)^{-\gamma}
$$

para todo $(\xi, \tau)=\left(\xi_{1}, \ldots, \xi_{N}, \tau\right) \in \mathbb{Z}^{N+1} \backslash\{0\}$

Teorema 3.1. O operador L dado por (3.1) é globalmente hipoelítico se, e somente se, as seguintes condições estão satisfeitas:

(1) as funções $b_{j}$ ñ̃o mudam de sinal, $j=1, \ldots, N$;

(2) $\operatorname{dim} \operatorname{span}\left\{b_{1}, \ldots, b_{N}\right\} \leqslant 1$;

(3) o par $\left(\alpha_{0}, \beta_{0}\right)$ satisfaz (DC1).

Já observamos que a condição $(\mathcal{P})$ é uma condição necessária para a hipoeliticidade global de $L$. Mais ainda, a hipoeliticidade global de $L$ implica que $L$ é globalmente resolúvel (ver teoremas 2.6 e 3.1). Tal fato está de acordo com um resultado geral envolvendo operadores no toro que satisfazem ${ }^{t} L=-L$ (ver Lema 1.2).

Comparando o Teorema 3.1 com a Observação 2.26, vemos que as condições que determinam a hipoeliticidade global de $L$ são as mesmas que determinam a resolubilidade forte.

Do Teorema 3.1 segue o seguinte resultado sobre a hipoeliticidade global de operadores com coeficientes constantes.

Corolário 3.2. Considere em $\mathbb{T}^{N+1}$ o operador

$$
\frac{\partial}{\partial t}+\sum_{j=1}^{N}\left(\alpha_{j}+i \beta_{j}\right) \frac{\partial}{\partial x_{j}}
$$

onde $\alpha_{j}$ e $\beta_{j}$ são números reais, para $j=1, \ldots, N$. Tal operador é globalmente hipoelítico se, e somente se, o par $(\alpha, \beta)$ satisfaz $(\mathrm{DC} 1)$, onde $\alpha=\left(\alpha_{1}, \ldots, \alpha_{N}\right)$ e $\beta=\left(\beta_{1}, \ldots, \beta_{N}\right)$.

Como corolário do Teorema 3.1 também obtemos o seguinte resultado.

Teorema 3.3 ([Ho]-Teorema 2.2). Sejam a e b funções em $\mathcal{C}^{\infty}\left(\mathbb{T}^{1}, \mathbb{R}\right)$ e considere em $\mathbb{T}_{(x, t)}^{2}$ o seguinte operador

$$
\frac{\partial}{\partial t}+(a(t)+i b(t)) \frac{\partial}{\partial x}
$$


Tal operador é globalmente hipoelítico se, e somente se, ocorre uma das seguintes situações:

(i) $b \equiv 0$ e $a_{0}=(2 \pi)^{-1} \int_{0}^{2 \pi} a(t) d t$ é um número irracional não-Liouville.

(ii) $b \not \equiv 0$ e $b$ ñ̃o muda de sinal.

Antes de entrarmos na demonstração do Teorema 3.1, consideremos o seguinte exemplo, que mostra a relação entre vetores de Liouville e a condição (DC1).

Exemplo 3.4. Sejam $a \in \mathcal{C}^{\infty}\left(\mathbb{T}^{1}, \mathbb{R}\right)$ e $b \in \mathcal{C}^{\infty}\left(\mathbb{T}^{1}, \mathbb{R}\right) \backslash\{0\}$, onde $b$ não muda de sinal e sejam $\lambda_{1}$ e $\lambda_{2}$ dois números reais. Afirmamos que o operador

$$
\frac{\partial}{\partial t}+\left(a(t)+i \lambda_{1} b(t)\right) \frac{\partial}{\partial x_{1}}+i \lambda_{2} b(t) \frac{\partial}{\partial x_{2}}
$$

é globalmente hipoelítico se, e somente se:

- $\lambda_{2} \neq 0,\left(a_{0}, \lambda_{1} / \lambda_{2}\right) \in \mathbb{R}^{2} \backslash \mathbb{Q}^{2}$ e $\left(a_{0}, \lambda_{1} / \lambda_{2}\right)$ não é um vetor de Liouville.

(compare com o Exemplo 2.11).

De fato, é fácil ver que o par $\left(\left(a_{0}, 0\right), b_{0}\left(\lambda_{1}, 0\right)\right)$ não satisfaz (DC1), logo $L$ não é globalmente hipoelítico quando $\lambda_{2}=0$. Suponha agora que $\lambda_{2} \neq 0$. Segue do Exemplo 2.11 que o operador $L$ não é globalmente resolúvel quando $\left(a_{0}, \lambda_{1} / \lambda_{2}\right)$ é um vetor de Liouville, $\log o L$ não é globalmente hipoelítico (ver Lema 1.2). Por outro lado, quando $\left(a_{0}, \lambda_{1} / \lambda_{2}\right)$ não é um vetor de Liouville, mostramos no Exemplo 2.11 que o par $\left(\left(a_{0}, 0\right), b_{0}\left(\lambda_{1}, \lambda_{2}\right)\right)$ satisfaz (DC2), na forma $(2.14)$, a qual é equivalente a (DC1) se, e somente se, $\left(a_{0}, \lambda_{1} / \lambda_{2}\right) \in \mathbb{R}^{2} \backslash \mathbb{Q}^{2}$. Por fim, o resultado segue do Teorema 3.1.

Vamos agora demonstrar o Teorema 3.1. Tal demonstração também será dividida em uma sequência de lemas e proposições. As proposições 3.5, 3.6 e 3.7 mostram que as condições (1)-(3) são necessárias para a hipoeliticidade global de $L$, enquanto que a Proposição 3.8 mostra que tais condições são também suficientes.

Como no Capítulo 2, utilizamos a conjugação dada pelo Lema 2.16, de modo que o estudo da hipoeliticidade global de (3.1) se reduz ao estudo da hipoeliticidade global de

$$
L=\frac{\partial}{\partial t}+\sum_{j=1}^{N}\left(a_{j 0}+i b_{j}(t)\right) \frac{\partial}{\partial x_{j}}
$$

onde a parte real dos coeficientes é constante. 


\section{I Demonstração do Teorema 3.1: condições necessá- rias}

Iniciaremos demonstrando que as condições (1)-(3) no Teorema 3.1 são suficientes para a hipoeliticidade global de $L$.

Consideremos primeiro a condição (1). Veremos que $L$ não é globalmente hipoelítico quando alguma função $b_{j}$ muda de sinal.

Proposição 3.5. Se para algum $j \in\{1, \ldots, N\}$ a função $b_{j}$ muda de sinal, então $L$ (dado por (3.4)) não é globalmente hipoelítico.

Demonstração: Sem perda de generalidade, podemos supor que $j=1$, ou seja, que $b_{1}$ muda de sinal.

Iremos construir uma distribuição $\mu \in \mathcal{D}^{\prime}\left(\mathbb{T}^{N+1}\right) \backslash \mathcal{C}^{\infty}\left(\mathbb{T}^{N+1}\right)$ de modo que $L \mu \in$ $\mathcal{C}^{\infty}\left(\mathbb{T}^{N+1}\right)$.

Suponha primeiro que $b_{10}=0$ e $a_{10} \in \mathbb{Z}$. Neste caso, a função $\int_{0}^{t} b_{1}(\tau) d \tau$ pertence a $\mathcal{C}^{\infty}\left(\mathbb{T}^{1}\right)$ e podemos supor que $a_{10}=0$ (ver Lema 2.16). Seja $M=\max _{t \in \mathbb{T}^{1}} \int_{0}^{t} b_{1}(\tau) d \tau$ e para cada $n \in \mathbb{N}$, considere $\xi(n)=(n, 0, \ldots, 0) \in \mathbb{Z}^{N}$. Defina

$$
\hat{\mu}(\xi(n), t)=\exp \left\{n \int_{0}^{t} b_{1}(\tau) d \tau-n M\right\}
$$

a qual é uma função em $\mathcal{C}^{\infty}\left(\mathbb{T}^{1}\right)$. Note que $|\hat{\mu}(\xi(n), t)| \leqslant 1$, para todo $t \in \mathbb{T}^{1}$ e $n \in \mathbb{N}$. Assim, a sequência $\hat{\mu}(\xi(n), \cdot)$ é de crescimento lento (ver Capítulo 1), logo podemos definir uma distribuição em $\mathcal{D}^{\prime}\left(\mathbb{T}^{N+1}\right)$ por

$$
\mu=\sum_{n \in \mathbb{N}} \hat{\mu}(\xi(n), t) \otimes e^{i\langle\xi(n), x\rangle}
$$

Mais ainda, para cada $t_{0} \in \mathbb{T}^{1}$ tal que $\int_{0}^{t_{0}} b_{1}(\tau) d \tau=M$, temos $\hat{\mu}\left(\xi(n), t_{0}\right)=1$, o que significa que a sequência $\hat{\mu}(\xi(n), \cdot)$ não decai rapidamente, logo $\mu \notin \mathcal{C}{ }^{\infty}\left(\mathbb{T}^{N+1}\right)$. Cálculos diretos mostram que

$$
L \mu=\sum_{n \in \mathbb{N}}\left[\partial_{t} \hat{\mu}(\xi(n), t)-n b_{1}(t) \hat{\mu}(\xi(n), t)\right] e^{i\langle\xi(n), x\rangle}=0
$$

e, portanto, $L$ não é globalmente hipoelítico. 
Suponha agora que $b_{10}=0$ e $a_{10} \notin \mathbb{Z}$. Neste caso, o item (II) do Teorema 2.6 e o Lema 1.2 implicam que $L$ não é globalmente hipoelítico. Podemos também utilizar as técnicas da demonstração da Proposição 2.18 para concluir que $L$ não é globalmente hipoelítico. De fato, sob as hipóteses $b_{1} \not \equiv 0, b_{10}=0$ e $a_{10} \notin \mathbb{Z}$, considere uma sequência crescente de inteiros positivos $k_{n}>n$ tal que $k_{n} a_{10} \notin \mathbb{Z}$. Considere também

$$
H(s, t) \doteq \int_{t-s}^{t} b_{1}(\tau) d \tau \quad \text { e } \quad A \doteq H\left(s_{0}, t_{0}\right)=\max _{0 \leqslant s, t \leqslant 2 \pi} H(s, t)
$$

onde $A>0$ e podemos supor que $0<t_{0}, s_{0}, \sigma_{0}<2 \pi$, onde $\sigma_{0}=t_{0}-s_{0}$. Tome $\phi \in$ $\mathcal{C}_{c}^{\infty}\left(\left(\sigma_{0}-\delta, \sigma_{0}+\delta\right)\right)$ uma função tal que $0 \leqslant \phi(t) \leqslant 1$ (para todo $t$ ) e $\phi(t) \equiv 1$ em uma vizinhança de $\left[\sigma_{0}-\delta / 2, \sigma_{0}+\delta / 2\right]$, onde $\delta>0$ é pequeno o bastante para que $\left(\sigma_{0}-\delta, \sigma_{0}+\delta\right) \subset\left(0, t_{0}\right)$.

Para $\xi(n)=\left(k_{n}, 0, \ldots, 0\right) \in \mathbb{Z}^{N}$, defina $\hat{f}(\xi(n), \cdot)$ pela extensão $2 \pi$ - periódica da função

$$
\left(1-e^{-2 \pi i k_{n} a_{10}}\right) \phi(t) e^{-A k_{n}} e^{i k_{n} a_{10}\left(t_{0}-t\right)}, \quad t \in[0,2 \pi] .
$$

Para os demais $\xi \in \mathbb{Z}^{N}$, faça $\hat{f}(\xi, \cdot)=0$.

Como $A k_{n}>A n$, o fator $e^{-A k_{n}}$ garante que tal sequência de funções é rapidamente decrescente, logo define uma função em $\mathcal{C}^{\infty}\left(\mathbb{T}^{N+1}\right)$, a qual é dada por

$$
f\left(x_{1}, t\right)=\sum_{n \in \mathbb{N}} \hat{f}(\xi(n), t) e^{i k_{n} x_{1}}
$$

Como feito na primeira parte da demonstração da Proposição 2.18, verifica-se que a equação $L u=f$ não tem solução em $\mathcal{C}^{\infty}\left(\mathbb{T}^{N+1}\right)$. Por outro lado, as equações $(2.25)$ definem uma sequência de crescimento lento em $\mathcal{C}^{\infty}\left(\mathbb{T}^{1}\right)$, a qual recordamos ser

$$
\hat{\mu}(\xi(n), t)=\left(1-e^{-2 \pi i k_{n} a_{10}}\right)^{-1} \int_{0}^{2 \pi} \hat{f}(\xi(n), t-s) e^{k_{n}\left(H(s, t)-i s a_{10}\right)} d s
$$

Tal sequência é de crescimento lento, pois

$$
|\langle\hat{\mu}(\xi(n), \cdot), \theta\rangle| \leqslant\left|1-e^{-2 \pi i k_{n} a_{10}}\right|^{-1}(2 \pi)^{2}\|\theta\|_{\infty}\|\hat{f}(\xi(n), \cdot)\|_{\infty} e^{k_{n} A} \leqslant(2 \pi)^{2}\|\theta\|_{\infty}
$$

para toda $\theta \in \mathcal{C}^{\infty}\left(\mathbb{T}^{1}\right)$. Assim, a sequência $\hat{\mu}(\xi(n), \cdot)$ define uma distribuição em $\mathcal{D}^{\prime}\left(\mathbb{T}^{N+1}\right)$, dada por 


$$
\mu=\sum_{n \in \mathbb{N}} \hat{\mu}(\xi(n), t) \otimes e^{i k_{n} x_{1}},
$$

a qual satisfaz $L \mu=f$. Portanto, $L$ não é globalmente hipoelítico quando $b_{10}=0$ e $a_{10} \notin \mathbb{Z}$.

Falta ainda analisar o caso em que $b_{10} \neq 0$. A mudança de sinal de $b_{1}$ nos permite aplicar o item (III) do Teorema 2.6 e o Lema 1.2 para obter que $L$ não é globalmente hipoelítico. Entretanto, faremos também uma outra demonstração para este caso, seguindo a linha da demonstração da Proposição 2.21 .

Vamos construir uma função $f \in \mathcal{C}^{\infty}\left(\mathbb{T}^{N+1}\right)$, de modo que a equação $L \mu=f$ tenha solução em $\mathcal{D}^{\prime}\left(\mathbb{T}^{N+1}\right) \backslash \mathcal{C}^{\infty}\left(\mathbb{T}^{N+1}\right)$.

Considerando o operador $-L$ e fazendo a mudança de variáveis $(X, T)=(x,-t)$, podemos supor que $b_{10}<0$. Defina $H(s, t)=\int_{t-s}^{t} b_{1}(\tau) d \tau$ e $A=\max _{0 \leqslant, s, t \leqslant 2 \pi} H(s, t)=$ $H\left(s_{0}, t_{0}\right)$. Sem perda de generalidade podemos supor que $0<s_{0}, t_{0}, t_{0}-s_{0}<2 \pi$. Tome $\phi \in \mathcal{C}_{c}^{\infty}\left(\left(\sigma_{0}-\delta, \sigma_{0}+\delta\right)\right)$, uma função que satisfaz $0 \leqslant \phi(t) \leqslant 1$ (para todo $t$ ) e $\phi(t) \equiv 1$ em uma vizinhança de $\left[\sigma_{0}-\delta / 2, \sigma_{0}+\delta / 2\right]$, onde $\delta>0$ é suficientemente pequeno de modo que $\left(\sigma_{0}-\delta, \sigma_{0}+\delta\right) \subset\left(0, t_{0}\right)$.

Para cada $n \in \mathbb{N}$, seja $\xi(n)=(n, 0, \ldots, 0) \in \mathbb{Z}^{N}$ e defina $\hat{f}(\xi(n), \cdot) \in \mathcal{C}^{\infty}\left(\mathbb{T}^{1}\right)$ pela extensão $2 \pi$-periódica da função

$$
e^{-n A} \phi(t) e^{i n a_{10}\left(t_{0}-t\right)}, \quad t \in[0,2 \pi]
$$

Agora considere

$$
f(x, t)=\sum_{n \in \mathbb{N}} \hat{f}(\xi(n), t) e^{i\langle\xi(n), x\rangle}=\sum_{n \in \mathbb{N}} \hat{f}(\xi(n), t) e^{i n x_{1}}
$$

note que, como $b_{1}$ muda de sinal, temos $A>0, \log \hat{f}(\xi(n), \cdot)$ é uma sequência rapidamente decrescente e $f$ definida acima é uma função em $\mathcal{C}^{\infty}\left(\mathbb{T}^{N+1}\right)$.

Procedendo como na demonstração da Proposição 2.21, verifica-se que para tal $f$ a equação $L \mu=f$ não tem solução em $\mathcal{C}^{\infty}\left(\mathbb{T}^{N+1}\right)$, uma vez que a sequência

$$
\hat{\mu}(\xi(n), t)=\left(1-e^{2 \pi n\left(b_{10}-i a_{10}\right)}\right)^{-1} \int_{0}^{2 \pi} \hat{f}(\xi(n), t-s) e^{n H(s, t)} e^{-i s n a_{10}} d s
$$


não decresce rapidamente. Por outro lado, tal sequência é de crescimento lento, pois satisfaz $|\hat{\mu}(\xi(n), t)| \leqslant 2 \pi\left|1-e^{2 \pi n\left(b_{10}-i a_{10}\right)}\right|^{-1} \leqslant 2 \pi\left|1-e^{2 \pi b_{10}}\right|^{-1}$, uma vez que $b_{10}<0$; logo tal sequência define a distribuição

$$
\mu=\sum_{n \in \mathbb{N}} \hat{\mu}(\xi(n), t) \otimes e^{i\langle\xi(n), x\rangle}=\sum_{n \in \mathbb{N}} \hat{\mu}(\xi(n), t) \otimes e^{i n x_{1}},
$$

a qual satisfaz $L \mu=f$. Portanto, $L$ não é globalmente hipoelítico.

O que conclui a demonstração da Proposição 3.5.

Consideremos agora a condição (2). Veremos que $L$ não é globalmente hipoelítico quando dim $\operatorname{span}\left\{b_{1}, \ldots, b_{N}\right\} \geqslant 2$. Neste caso, note que existem duas funções $b_{m}$ e $b_{\ell}$ que são linearmente independentes. Novamente, podemos aplicar o Teorema 2.6 e o Lema 1.2, mas faremos outra demonstração, a qual segue a mesma técnica da demonstração da Proposição 2.21. Note que, pela Proposição 3.5 podemos assumir que as funções $b_{m}$ e $b_{\ell}$ não mudam de sinal.

Proposição 3.6. Suponha que existam dois índices $m$ e $\ell$ em $\{1, \ldots, N\}$ tais que as funções $b_{m}$ e $b_{\ell}$ são $\mathbb{R}$ - linearmente independentes e não mudam de sinal. Então o operador L (dado por (3.4)) não é globalmente hipoelítico.

Demonstração: Vamos construir uma função $f \in \mathcal{C}^{\infty}\left(\mathbb{T}^{N+1}\right)$ tal que a equação $L \mu=f$ tenha uma solução em $\mathcal{D}^{\prime}\left(\mathbb{T}^{N+1}\right) \backslash \mathcal{C}^{\infty}\left(\mathbb{T}^{N+1}\right)$. Após uma reordenação das variáveis $x_{1}, \ldots, x_{N}$, podemos supor que $m=1$ e $\ell=2$. Aplicando o Lema 2.2 obtemos inteiros não nulos tais que $\theta(t)=p b_{1}(t)+q b_{2}(t)$ muda de sinal e $\theta_{0}=p b_{10}+q b_{20}<0$. Repetindo os argumentos da demonstração da Proposição 2.21, construímos uma função $f \in \mathcal{C}^{\infty}\left(\mathbb{T}^{N+1}\right)$ tal que a equação $L \mu=f$ não tem solução $\mu$ em $\mathcal{C}^{\infty}\left(\mathbb{T}^{N+1}\right)$, a saber, $f$ é dada por

$$
f(x, t)=\sum_{n \in \mathbb{N}} \hat{f}(\xi(n), t) e^{i\langle\xi(n), x\rangle}
$$

onde, para cada $n \in \mathbb{N}, \xi(n)=(n p, n q, 0, \ldots, 0) \in \mathbb{Z}^{N}$ e $\hat{f}(\xi(n), \cdot)$ é a extensão $2 \pi$ periódica da função

$$
e^{-n A} \phi(t) e^{i n\left(p a_{10}+q a_{20}\right)\left(t_{0}-t\right)}, \quad t \in[0,2 \pi]
$$

sendo que:

- $H(s, t)=\int_{t-s}^{t} \theta(\tau) d \tau, s, t \in[0,2 \pi], A=H\left(s_{0}, t_{0}\right)=\max _{0 \leqslant s, t \leqslant 2 \pi} H(s, t)>0$ e $0<$ $s_{0}, t_{0}, \sigma_{0}<2 \pi$, onde $\sigma_{0}=t_{0}-s_{0}$; 
- $\phi \in \mathcal{C}_{c}^{\infty}\left(\left(\sigma_{0}-\delta, \sigma_{0}+\delta\right)\right)$ é uma função que satisfaz $0 \leqslant \phi(t) \leqslant 1$ (para todo $t$ ) e $\phi(t) \equiv 1$ em uma vizinhança de $\left[\sigma_{0}-\delta / 2, \sigma_{0}+\delta / 2\right]$, onde $\delta>0$ é suficientemente pequeno de modo que $\left(\sigma_{0}-\delta, \sigma_{0}+\delta\right) \subset\left(0, t_{0}\right)$.

Para concluir a demonstração, vamos exibir $\mu \in \mathcal{D}^{\prime}\left(\mathbb{T}^{N+1}\right) \backslash \mathcal{C}^{\infty}\left(\mathbb{T}^{N+1}\right)$ tal que $L \mu=f$. Para cada $n \in \mathbb{N}$, defina

$$
\hat{\mu}(\xi(n), t)=\left(1-e^{2 \pi n\left(\theta_{0}-i\left(p a_{10}+q a_{20}\right)\right)}\right)^{-1} \int_{0}^{2 \pi} \hat{f}(\xi(n), t-s) e^{-i s n\left(p a_{10}+q a_{20}\right)} e^{n \int_{t-s}^{t} \theta(\tau) d \tau} d s
$$

a qual é uma função em $\mathcal{C}^{\infty}\left(\mathbb{T}^{1}\right)$, para cada $n \in \mathbb{N}$ (ver Lema 1.6). Mais ainda,

$$
|\hat{\mu}(\xi(n), t)| \leqslant 2 \pi\left|1-e^{2 \pi n\left(\theta_{0}-i\left(p a_{10}+q a_{20}\right)\right)}\right|^{-1} \leqslant 2 \pi\left|1-e^{2 \pi n \theta_{0}}\right|^{-1} \leqslant 2 \pi\left|1-e^{2 \pi \theta_{0}}\right|^{-1},
$$

uma vez que $\theta_{0}<0$. Sendo assim, a sequência $\hat{\mu}(\xi(n), \cdot)$ é de crescimento lento, logo define uma distribuição em $\mathcal{D}^{\prime}\left(\mathbb{T}^{N+1}\right)$ dada por

$$
\mu=\sum_{n \in \mathbb{N}} \hat{\mu}(\xi(n), t) \otimes e^{i\langle\xi(n), x\rangle} .
$$

Do Lema 1.6 segue que cada coeficiente parcial de Fourier $\hat{\mu}(\xi(n), \cdot)$ de $\mu$ satisfaz a equação

$$
\partial_{t} \hat{\mu}(\xi(n), t)+i\left(\left(p a_{10}+q a_{20}\right)+i \theta(t)\right) \hat{\mu}(\xi(n), t)=\hat{f}(\xi(n), t),
$$

para todo $t \in \mathbb{T}^{1}$. Portanto, $L \mu=f$, o que conclui a demonstração.

Para finalizar a demonstração de que as condições (1)-(3) são necessárias para a hipoeliticidade global de $L$, consideramos agora a condição (3). Pelas proposições 3.5 e 3.6 , podemos supor que as funções $b_{j}$ não mudam de sinal e que $\operatorname{dim} \operatorname{span}\left\{b_{1}, \ldots, b_{N}\right\} \leqslant$ 1, ou seja, podemos supor que o operador $L$ é da forma

$$
L=\frac{\partial}{\partial t}+\sum_{j=1}^{N}\left(a_{j 0}+i \lambda_{j} b(t)\right) \frac{\partial}{\partial x_{j}}
$$

onde $b \in \mathcal{C}^{\infty}\left(\mathbb{T}^{1}, \mathbb{R}\right), b$ não muda de sinal e $\lambda \doteq\left(\lambda_{1}, \ldots, \lambda_{N}\right) \in \mathbb{R}^{N} \backslash\{0\}$. Lembre-se que $\alpha_{0}=\left(a_{10}, \ldots, a_{N 0}\right)$ e $b_{0}=(2 \pi)^{-1} \int_{0}^{2 \pi} b(t) d t$.

Proposição 3.7. Seja L o operador dado por (3.6). Se o par $\left(\alpha_{0}, b_{0} \lambda\right) \in \mathbb{R}^{N} \times \mathbb{R}^{N}$ não satisfaz a condição Diofantina (DC1), então L não é globalmente hipoelítico. 
Demonstração: Uma vez que o par $\left(\alpha_{0}, b_{0} \lambda\right) \in \mathbb{R}^{N} \times \mathbb{R}^{N}$ não satisfaz (DC1), uma das seguintes situações ocorre:

(i) existe uma sequência $\left(\xi(n), \tau_{n}\right)_{n \in \mathbb{N}} \subset \mathbb{Z}^{N} \times \mathbb{Z}$ tal que $|\xi(n)|+\left|\tau_{n}\right| \geqslant n,\left\langle\xi(n), b_{0} \lambda\right\rangle=0$ e $\tau_{n}=-\left\langle\xi(n), \alpha_{0}\right\rangle \in \mathbb{Z}$, para todo $n \in \mathbb{N}$.

(ii) o par $\left(\alpha_{0}, b_{0} \lambda\right)$ não satisfaz (DC2).

Suponha que ocorre (i). Note que a sequência $|\xi(n)|$ não pode ser limitada. Assim, tomando uma subsequência podemos assumir que $|\xi(n)|$ é crescente. Defina

$$
\mu=\sum_{n=1}^{\infty} e^{-i t\left\langle\xi(n), \alpha_{0}\right\rangle} e^{i\langle\xi(n), x\rangle}
$$

note que $e^{-i t\left\langle\xi(n), \alpha_{0}\right\rangle} \in \mathcal{C}^{\infty}\left(\mathbb{T}^{1}\right)$ e $\left|e^{-i t\left\langle\xi(n), \alpha_{0}\right\rangle}\right|=1$, logo $\mu \in \mathcal{D}^{\prime}\left(\mathbb{T}^{N+1}\right) \backslash \mathcal{C}^{\infty}\left(\mathbb{T}^{N+1}\right)$. Mais ainda, um cálculo direto mostra que

$$
L \mu=\sum_{n=1}^{\infty}\left(-i\left\langle\xi(n), \alpha_{0}\right\rangle+i\left\langle\xi(n), \alpha_{0}+i b(t) \lambda\right\rangle\right) e^{i\langle\xi(n), x\rangle}=0
$$

pois $\langle\xi(n), b(t) \lambda\rangle \equiv 0$, uma vez que $b$ não muda de sinal e $\left\langle\xi(n), b_{0} \lambda\right\rangle=0$. Poranto, $L$ não é globalmente hipoelítico.

Suponha agora que ocorre (ii). Com o objetivo de construir uma função $f \in$ $\mathcal{C}^{\infty}\left(\mathbb{T}^{N+1}\right)$ tal que $L \mu=f$ tenha uma solução em $\mathcal{D}^{\prime}\left(\mathbb{T}^{N+1}\right) \backslash \mathcal{C}^{\infty}\left(\mathbb{T}^{N+1}\right)$, vamos proceder como na demonstração da Proposição 2.24. Do Lema 2.23 segue que o par $\left(\alpha_{0}, b_{0} \lambda\right)$ não satisfaz (DC3), logo existe uma sequência $(\xi(n))_{n \in \mathbb{N}} \subset \mathbb{Z}^{N}$ tal que $|\xi(n+1)|>|\xi(n)|>n$, $\left\langle\xi(n), b_{0} \lambda-i \alpha_{0}\right\rangle \notin i \mathbb{Z} \mathrm{e}$

$$
\left|1-\exp \left\{2 \pi\left\langle\xi(n), b_{0} \lambda-i \alpha_{0}\right\rangle\right\}\right|<|\xi(n)|^{-n}
$$

para todo $n \in \mathbb{N}$. Tomando uma subsequência e substituindo $\xi(n)$ por $-\xi(n)$, se necessário, podemos supor que ocorre uma das seguintes situações:

(ii.1) $\left\langle\xi(n), b_{0} \lambda\right\rangle>0 \mathrm{e}$

$$
\left|1-\exp \left\{2 \pi\left\langle\xi(n), b_{0} \lambda-i \alpha_{0}\right\rangle\right\}\right|<|\xi(n)|^{-n}<n^{-n}, \text { para todo } n \in \mathbb{N}
$$


(ii.2) $\left\langle\xi(n), b_{0} \lambda\right\rangle=0,\left\langle\xi(n), \alpha_{0}\right\rangle \notin \mathbb{Z} \mathrm{e}$

$$
\left|1-\exp \left\{-2 \pi i\left\langle\xi(n), \alpha_{0}\right\rangle\right\}\right|<|\xi(n)|^{-n}<n^{-n} \text {, para todo } n \in \mathbb{N} \text {. }
$$

Vamos mostrar que $L$ não é globalmente hipoelítico quando ocorre (ii.1) ou (ii.2).

Suponha primeiro que ocorre (ii.1).

Tome $\delta>0$ suficientemente pequeno de modo que $\left(\frac{\pi}{2}-\delta, \frac{\pi}{2}+\delta\right) \subset(0, \pi)$ e seja $\phi$ uma função em $\mathcal{C}_{c}^{\infty}\left(\left(\frac{\pi}{2}-\delta, \frac{\pi}{2}+\delta\right)\right)$ tal que $0 \leqslant \phi(t) \leqslant 1$ (para todo $t$ ) e $\phi \equiv 1$ em uma vizinhança de $\left[\frac{\pi-\delta}{2}, \frac{\pi+\delta}{2}\right]$.

Seja $\hat{f}(\xi(n), \cdot)$ a extensão $2 \pi$ - periódica de

$$
\left(1-\exp \left\{2 \pi\left\langle\xi(n), b_{0} \lambda-i \alpha_{0}\right\rangle\right\}\right) e^{-\left\langle\xi(n), b_{0} \lambda\right\rangle} \phi(t) e^{i(\pi-t)\left\langle\xi(n), \alpha_{0}\right\rangle}, \quad t \in[0,2 \pi]
$$

O decaimento rápido de tal sequência é garantido pela estimativa (3.8), uma vez que $\left\langle\xi(n), b_{0} \lambda\right\rangle>0$; logo a função $f$ dada por

$$
f(x, t)=\sum_{n \in \mathbb{N}} \hat{f}(\xi(n), t) e^{i\langle\xi(n), x\rangle}
$$

pertence a $\mathcal{C}^{\infty}\left(\mathbb{T}^{N+1}\right)$.

Afirmamos que existe $\mu \in \mathcal{D}^{\prime}\left(\mathbb{T}^{N+1}\right) \backslash \mathcal{C}^{\infty}\left(\mathbb{T}^{N+1}\right)$ tal que $L \mu=f$. De fato, tal distribuição $\mu$ é dada por

$$
\mu=\sum_{n \in \mathbb{N}} \hat{\mu}(\xi(n), t) \otimes e^{i\langle\xi(n), x\rangle}
$$

onde

$$
\hat{\mu}(\xi(n), t)=\left(1-e^{2 \pi c_{n}}\right)^{-1} \int_{0}^{2 \pi} \hat{f}(\xi(n), t-s) e^{\langle\xi(n), \lambda\rangle \int_{t-s}^{t} b(\tau) d \tau} e^{-i s\left\langle\xi(n), \alpha_{0}\right\rangle} d s
$$

sendo que definimos $c_{n} \doteq\left\langle\xi(n), b_{0} \lambda-i \alpha_{0}\right\rangle$ para simplificar a notação. Para cada $n \in \mathbb{N}$, $\hat{\mu}(\xi(n), \cdot)$ é a função em $\mathcal{C}^{\infty}\left(\mathbb{T}^{1}\right)$ que satisfaz a equação

$$
\partial_{t} \hat{\mu}(\xi(n), t)+i\left\langle\xi(n), \alpha_{0}+i b(t) \lambda\right\rangle \hat{\mu}(\xi(n), t)=\hat{f}(\xi(n), t) \quad(\text { ver Lema 1.6). }
$$

Mais ainda, como $\langle\xi(n), \lambda\rangle\left(\int_{t-s}^{t} b(\tau) d \tau-b_{0}\right) \leqslant 0$, da definição de $\hat{f}(\xi(n), \cdot)$ obtemos $|\hat{u}(\xi(n), t)| \leqslant 2 \pi$, para todo $t \in \mathbb{T}^{1}$ e $n \in \mathbb{N}$. Assim, $\mu$ definida em (3.10) de fato está em $\mathcal{D}^{\prime}\left(\mathbb{T}^{N+1}\right)$ e satisfaz $L \mu=f$. Resta ver que $\mu \notin \mathcal{C}^{\infty}\left(\mathbb{T}^{N+1}\right)$. Para tanto, note que 
$|\hat{u}(\xi(n), \pi)| \geqslant \delta e^{-\left\langle\xi(n), b_{0} \lambda\right\rangle} \geqslant \delta / 2$, pois de $(3.8)$ temos $\lim _{n \rightarrow \infty}\left\langle\xi(n), b_{0} \lambda\right\rangle=0$. Portanto, $L$ não é globalmente hipoelítico quando ocorre (ii.1).

Suponha agora que ocorre (ii.2). Lembre-se que $\left\langle\xi(n), b_{0} \lambda\right\rangle=0$ e $b$ não muda de sinal, $\log 0\langle\xi(n), b(t) \lambda\rangle \equiv 0$, para todo $n \in \mathbb{N}$. Neste caso, considere $f$ dada por

$$
f(x, t)=\sum_{n \in \mathbb{N}} \hat{f}(\xi(n), t) e^{i\langle\xi(n), x\rangle},
$$

onde $\hat{f}(\xi(n), \cdot)$ é a extensão $2 \pi$ - periódica de

$$
\left(1-e^{-2 \pi i\left\langle\xi(n), \alpha_{0}\right\rangle}\right) \phi(t) e^{i(\pi-t)\left\langle\xi(n), \alpha_{0}\right\rangle}, \quad t \in[0,2 \pi]
$$

sendo que $\phi$ é a mesma do caso (ii.1). O decaimento rápido de $\hat{f}(\xi(n), \cdot)$ vem de $(3.9)$, $\operatorname{logo} f \in \mathcal{C}^{\infty}\left(\mathbb{T}^{N+1}\right)$.

Para tal $f$, considere a distribuição

$$
\mu=\sum_{n \in \mathbb{N}} \hat{\mu}(\xi(n), t) \otimes e^{i\langle\xi(n), x\rangle},
$$

onde

$$
\hat{\mu}(\xi(n), t)=\left(1-e^{-2 \pi\left\langle\xi(n), \alpha_{0}\right\rangle}\right)^{-1} \int_{0}^{2 \pi} \hat{f}(\xi(n), t-s) e^{-i s\left\langle\xi(n), \alpha_{0}\right\rangle} d s
$$

note que $\hat{\mu}(\xi(n), \cdot)$ é a função em $\mathcal{C}^{\infty}\left(\mathbb{T}^{1}\right)$ que satisfaz a equação

$$
\partial_{t} \hat{\mu}(\xi(n), t)+i\left\langle\xi(n), \alpha_{0} \lambda\right\rangle \hat{\mu}(\xi(n), t)=\hat{f}(\xi(n), t) \quad(\text { ver Lema 1.6). }
$$

Como no caso anterior, temos $|\hat{\mu}(\xi(n), t)| \leqslant 2 \pi$, para todo $t \in \mathbb{T}^{1}$ e $n \in \mathbb{N}$, bem como $|\hat{\mu}(\xi(n), \pi)| \geqslant \delta$, para todo $n \in \mathbb{N}$. Portanto, $\mu \in \mathcal{D}^{\prime}\left(\mathbb{T}^{N+1}\right) \backslash \mathcal{C}^{\infty}\left(\mathbb{T}^{N+1}\right)$ e $L \mu=f$, ou seja, $L$ também não é globalmente hipoelítico quando ocorre (ii.2).

O que conclui a demonstração da Proposição 3.7.

\section{II Demonstração do Teorema 3.1: condições suficien- tes}

Nesta seção, mostraremos que as condições (1)-(3) no Teorema 3.1 são suficientes para a hipoeliticidade global de $L$. Para tanto, podemos supor que $L$ é dado por (3.4); 
mais ainda, sob as condições (1)-(2), um operador da forma (3.4) pode ser reescrito na forma

$$
L=\frac{\partial}{\partial t}+\sum_{j=1}^{N}\left(a_{j 0}+i \lambda_{j} b(t)\right) \frac{\partial}{\partial x_{j}},
$$

onde $b \in \mathcal{C}^{\infty}\left(\mathbb{T}^{1}, \mathbb{R}\right), b$ não muda de sinal e $\lambda \doteq\left(\lambda_{1}, \ldots, \lambda_{N}\right) \in \mathbb{R}^{N} \backslash\{0\}$. Supondo que o par $\left(\alpha_{0}, b_{0} \lambda\right)$ satisfaz a condição Diofantina (DC1), mostraremos que (3.11) é globalmente hipoelítico, concluindo, assim, a demonstração do Teorema 3.1.

Proposição 3.8. Seja L o operador dado por (3.11). Se $\left(\alpha_{0}, b_{0} \lambda\right)$ satisfaz (DC1), então $L$ é globalmente hipoelítico.

Demonstração: Considere $\mu \in \mathcal{D}^{\prime}\left(\mathbb{T}^{N+1}\right)$ uma distribuição tal que $L \mu=f$, com $f \in \mathcal{C}^{\infty}\left(\mathbb{T}^{N+1}\right)$. Mostraremos que $\mu \in \mathcal{C}^{\infty}\left(\mathbb{T}^{N+1}\right)$. Para tanto, utilizamos série parcial de Fourier nas variáveis $\left(x_{1}, \ldots, x_{N}\right)$, a qual nos permite escrever

$$
\mu=\sum_{\xi \in \mathbb{Z}^{N}} \hat{\mu}(\xi, \cdot) \otimes e^{i\langle\xi, x\rangle}
$$

onde $\hat{\mu}(\xi, \cdot) \in \mathcal{D}^{\prime}\left(\mathbb{T}^{1}\right)$ e

$$
f(x, t)=\sum_{\xi \in \mathbb{Z}^{N}} \hat{f}(\xi, t) \otimes e^{i\langle\xi, x\rangle}
$$

$\operatorname{com} \hat{f}(\xi, \cdot) \in \mathcal{C}^{\infty}\left(\mathbb{T}^{1}\right)$.

A série parcial de Fourier nos leva às seguintes equações

$$
\partial_{t} \hat{\mu}(\xi, \cdot)+i\left\langle\xi, \alpha_{0}+i b(\cdot) \lambda\right\rangle \hat{\mu}(\xi, \cdot)=\hat{f}(\xi, \cdot)
$$

onde tal igualdade é no espaço $\mathcal{D}^{\prime}\left(\mathbb{T}^{1}\right)$.

Como as soluções de (3.12) dependem de propriedades do coeficiente $i\left\langle\xi, \alpha_{0}+i b(t) \lambda\right\rangle$, a demonstração naturalmente se divide em três casos.

Suponha que $\xi \in \mathbb{Z}^{N}$ é tal que $\left\langle\xi, b_{0} \lambda\right\rangle=0$ e $\left\langle\xi, \alpha_{0}\right\rangle \in \mathbb{Z}$. Note que, como vale (DC1), isso só pode ocorrer para $\xi=0 \in \mathbb{Z}^{N}$. Deste modo, obtemos a equação $\partial_{t} \hat{\mu}(0, \cdot)=$ $\hat{f}(0, \cdot)$, a qual implica que $\int_{0}^{2 \pi} \hat{f}(0, t) d t=0$ e que $\hat{\mu}(0, \cdot) \in \mathcal{C}^{\infty}\left(\mathbb{T}^{1}\right)$ é dada por $\hat{\mu}(0, t)=$ $\int_{0}^{t} \hat{f}(0, s) d s+C$, onde $C \in \mathbb{C}$.

Suponha agora que $\xi \in \mathbb{Z}^{N}$ é tal que $\left\langle\xi, b_{0} \lambda\right\rangle \neq 0$. Neste caso, cada equação (3.12) possui uma única solução em $\mathcal{C}^{\infty}\left(\mathbb{T}^{1}\right)$, a qual pode ser escrita de duas maneiras distintas (ver Lema 1.6). Para simplificar a notação, defina $c_{\xi}=\left\langle\xi, b_{0} \lambda-i \alpha_{0}\right\rangle$. 
Se $\left\langle\xi, b_{0} \lambda\right\rangle<0$, escrevemos a solução de $(3.12)$ por

$$
\hat{\mu}(\xi, t)=\left(1-e^{2 \pi c_{\xi}}\right)^{-1} \int_{0}^{2 \pi} \hat{f}(\xi, t-s) e^{\langle\xi, \lambda\rangle \int_{t-s}^{t} b(\tau) d \tau} e^{-i s\left\langle\xi, \alpha_{0}\right\rangle} d s, t \in \mathbb{T}^{1} .
$$

Por outro lado, quando $\left\langle\xi, b_{0} \lambda\right\rangle>0$, escrevemos a solução de (3.12) por

$$
\hat{\mu}(\xi, t)=\left(e^{-2 \pi c_{\xi}}-1\right)^{-1} \int_{0}^{2 \pi} \hat{f}(\xi, t+s) e^{-\langle\xi, \lambda\rangle \int_{t}^{t+s} b(\tau) d \tau} e^{i s\left\langle\xi, \alpha_{0}\right\rangle} d s, t \in \mathbb{T}^{1} .
$$

Considere agora os índices $\xi \in \mathbb{Z}^{N}$ tais que $\left\langle\xi, b_{0} \lambda\right\rangle=0$ e $\left\langle\xi, \alpha_{0}\right\rangle \notin \mathbb{Z}$. Como $b$ não muda de sinal, temos $\langle\xi, b(t) \lambda\rangle \equiv 0$, logo a equação (3.12) se reduz a

$$
\partial_{t} \hat{\mu}(\xi, \cdot)+i\left\langle\xi, \alpha_{0}\right\rangle \hat{\mu}(\xi, \cdot)=\hat{f}(\xi, \cdot)
$$

a qual (como no caso anterior) possui uma única solução em $\mathcal{C}^{\infty}\left(\mathbb{T}^{1}\right)$. Escrevemos tal solução por

$$
\hat{\mu}(\xi, t)=\left(1-e^{-2 \pi i\left\langle\xi, \alpha_{0}\right\rangle}\right)^{-1} \int_{0}^{2 \pi} \hat{f}(\xi, t-s) e^{-i s\left\langle\xi, \alpha_{0}\right\rangle} d s, t \in \mathbb{T}^{1} .
$$

Por fim, procedendo como na Proposição 2.25 obtemos o decaimento rápido da sequência $(\hat{\mu}(\xi, \cdot))_{\xi \in \mathbb{Z}^{N}} \subset \mathcal{C}^{\infty}\left(\mathbb{T}^{1}\right)$, uma vez que (DC1) implica (DC2). Portanto, $\mu \in$ $\mathcal{C}^{\infty}\left(\mathbb{T}^{N+1}\right)$. 



\section{Outro operador com imagem fechada e de codimensão infinita}

Este capítulo trata da resolubilidade global da seguinte classe de campos vetoriais em $\mathbb{T}^{3}$ :

$$
\mathbf{L}=\frac{\partial}{\partial t}+(a(x)+i b(x))\left(\frac{\partial}{\partial x}+\lambda \frac{\partial}{\partial y}\right)
$$

onde denotamos por $(x, y, t)$ as coordenadas em $\mathbb{T}^{3} ;$ as funções $a$ e $b$ estão em $\mathcal{C}\left(\mathbb{T}^{1}, \mathbb{R}\right)$ e $\lambda$ é um número real. Utilizaremos a notação $c(x)=a(x)+i b(x)$.

Quando $\lambda=0$, obtemos o campo vetorial

$$
\frac{\partial}{\partial t}+(a(x)+i b(x)) \frac{\partial}{\partial x}
$$

A resolubilidade forte de tal campo, visto como um campo em $\mathbb{T}_{(x, t)}^{2}$, foi abordada nos trabalhos [BD1] e [D2]. Com o objetivo de apresentar os resultados sobre tal resolubilidade, denote por $n_{\ell} \geqslant 1$ e por $m_{\ell} \geqslant 1$ a ordem de anulamento das funções $a$ e $b$, respectivamente, em cada zero $x_{\ell} \in c^{-1}(0)$.

O operador (em $\mathbb{T}^{2}$ ) dado por (4.2) não é fortemente resolúvel quando ocorrer alguma das seguintes situações: (ver [BD1], [D2] e [H6]-Corolário 26.4.8)

- $c(x) \doteq a(x)+i b(x)$ se anula de ordem infinita em algum ponto;

- (4.2) não satisfaz a condição $(\mathcal{P})$, o que significa que $b$ muda de sinal ao longo de uma curva integral do campo $\partial_{t}+a(x) \partial_{x}$; equivalentemente, $b$ muda de sinal entre dois zeros consecutivos de $c$.

- $b \equiv 0$ em algum intervalo $\left(x_{\ell}, x_{\ell+1}\right)$, onde $x_{\ell}$ e $x_{\ell+1}$ são zeros consecutivos de $c$; 
- $x_{\ell} \in c^{-1}(0), n_{\ell}<\infty, m_{\ell} \geqslant 2 n_{\ell}-1$ e $m_{\ell} \geqslant 2$;

- $x_{\ell} \in c^{-1}(0)$ e $m_{\ell}=1$.

Por outro lado, a seguinte condição é suficiente para a resolubilidade forte do operador $\left(\right.$ em $\left.\mathbb{T}^{2}\right)$ dado por (4.2): (ver [BD1] e [D2])

- (4.2) satisfaz a condição $(\mathcal{P}), c$ não possui zeros de ordem infinita e em cada $x_{\ell} \in c^{-1}(0)$, temos: $m_{\ell}<\infty$ e $2 \leqslant m_{\ell}<2 n_{\ell}-1$.

A estratégia empregada no estudo da resolubilidade forte de (4.2) (em $\left.\mathbb{T}^{2}\right)$ foi utilizar a redução feita em [BCP]-Teorema 3.1, que transforma o estudo da resolubilidade forte no estudo da resolubilidade em uma vizinhança de $\left\{x_{\ell}\right\} \times \mathbb{T}^{1}$, para cada $x_{\ell} \in\left\{x_{1}<\right.$ $\left.\cdots<x_{N}\right\}=c^{-1}(0)$. Entretanto, quando $c^{-1}(0) \neq \emptyset$, veremos que os operadores do tipo (4.1) não são fortemente resolúveis, logo não podemos aplicar a estratégia anterior para obter resultados sobre a resolubilidade global de (4.1). O que faremos é utilizar a técnica de $[\mathrm{BP}]$, a qual consiste em utilizar série parcial de Fourier em $t$ e resolver uma sequência de edo's, as quais são resolvidas inicialmente em cada aberto $\left(x_{\ell}, x_{\ell+1}\right)$ e depois esses pedaços de soluções são colados de modo a obter uma solução em $\mathbb{T}^{1}$. Em $[\mathrm{BP}]$ os campos possuem coeficientes reais, o que foi fundamental no controle do decaimento desta sequência de soluções obtida pelo método descrito acima. Para os operadores $L$ do tipo (4.1), a parte imaginária do coeficiente, ou seja, a função $b$, influenciará no controle do decaimento. Certas relações entre as ordens de anulamento das funções $a$ e $b$ (como em [BD1]) nos permitirão obter decaimento rápido. Outro ponto importante é que agora não vamos mais supor que o operador satisfaz a condição $(\mathcal{P})$, uma vez que tal condição não é necessária para a resolubilidade global; assim, a função $b$ poderá mudar de sinal entre dois zeros consecutivos de $c$. Será interessante detectar a quantidade de vezes que a função $b$ pode mudar de sinal entre dois zeros consecutivos de $c$, de modo que valha a resolubilidade global de L.

Após os comentários introdutórios acima, iniciamos agora a análise da resolubilidade global de $L$ (dado por (4.1)), considerando primeiro o caso em que $c^{-1}(0)=\emptyset$. Para os operadores (4.2) em $\mathbb{T}^{2}$, este caso era uma consequência dos teoremas sobre resolubilidade de Hörmander em [H3]. Para L, neste caso em que $c^{-1}(0)=\emptyset$, a resolubilidade global será como no caso tubo. 
Defina

$$
\alpha \doteq(2 \pi)^{-1} \int_{0}^{2 \pi} \frac{a(x)}{a(x)^{2}+b(x)^{2}} d x, \quad \beta \doteq-(2 \pi)^{-1} \int_{0}^{2 \pi} \frac{b(x)}{a(x)^{2}+b(x)^{2}} d x
$$

e considere a seguinte condição Diofantina para uma tripla $\left(\lambda_{1}, \lambda_{2}, \lambda_{3}\right) \in \mathbb{R}^{3}$ :

(DC4) existem constantes $C>0$ e $\gamma>0$ tais que

$$
\left|\tau+j\left(\lambda_{1}+i \lambda_{2}\right)+k \lambda_{3}\right| \geqslant C(|j|+|k|+|\tau|)^{-\gamma}
$$

para todo $(j, k, \tau) \in \mathbb{Z}^{3}$ tal que $\tau+j\left(\lambda_{1}+i \lambda_{2}\right)+k \lambda_{3} \neq 0$; tal condição é a condição (DC2) para o par $\left(\left(\lambda_{1}, \lambda_{3}\right),\left(\lambda_{2}, 0\right)\right) \in \mathbb{R}^{2} \times \mathbb{R}^{2}$.

Teorema 4.1. Seja L o operador dado por (4.1) e suponha que $c^{-1}(0)=\emptyset$. Neste caso, $\mathrm{L}$ é globalmente resolúvel se, e somente se, ocorre uma das seguintes situações:

(i) $b \equiv 0 e(\alpha, 0, \lambda)$ satisfaz (DC4).

(ii) $b \not \equiv 0, \beta=0, \alpha \in \mathbb{Z}, \lambda \in \mathbb{Z}$ e todos os subniveis

$$
\left\{x \in \mathbb{T}^{1} ; \int_{0}^{x} \frac{b(\tau)}{a(\tau)^{2}+b(\tau)^{2}} d \tau<r\right\}(r \in \mathbb{R})
$$

são conexos.

(iii) $b \not \equiv 0, \beta \neq 0, b$ não muda de sinal $e(\alpha, \beta, \lambda)$ satisfaz (DC4).

Demonstração: Como $c^{-1}(0)=\emptyset$, afirmamos que o operador $L$ é globalmente resolúvel se, e somente se, o operador

$$
\mathrm{L}_{1 / c} \doteq \frac{1}{c(x)} \mathrm{L}=\frac{1}{c(x)} \frac{\partial}{\partial t}+\frac{\partial}{\partial x}+\lambda \frac{\partial}{\partial y}
$$

é globalmente resolúvel. De fato, temos $g \in\left(\operatorname{ker}^{t} \mathrm{~L}_{1 / c}\right)^{\circ} \backslash \mathrm{L}_{1 / c} \mathcal{C}^{\infty}\left(\mathbb{T}^{3}\right)$ se, e somente se, $c g \in\left(\operatorname{ker}^{t} \mathrm{~L}\right)^{\circ} \backslash \mathrm{LC} \mathcal{C}^{\infty}\left(\mathbb{T}^{3}\right)$. Por fim, efetuando a mudança de variáveis $(X, Y, T)=(t, y, x)$ segue que a resolubilidade de $\mathrm{L}$ é equivalente à resolubilidade de

$$
\frac{\partial}{\partial T}+\frac{1}{c(T)} \frac{\partial}{\partial X}+\lambda \frac{\partial}{\partial Y}=\frac{\partial}{\partial T}+\frac{a(T)-i b(T)}{a^{2}(T)+b^{2}(T)} \frac{\partial}{\partial X}+\lambda \frac{\partial}{\partial Y}
$$

a qual segue do Teorema 2.6. 
Exemplo 4.2. O item (ii) do Teorema 4.1 implica que o operador

$$
\frac{\partial}{\partial t}+(1+i \operatorname{sen}(x))\left(\frac{\partial}{\partial x}+\frac{\partial}{\partial y}\right)
$$

não é globalmente resoluvel, uma vez que

$$
\int_{0}^{2 \pi} \frac{\operatorname{sen}(\tau)}{1+\operatorname{sen}^{2}(\tau)} d \tau=0
$$

e

$$
\frac{1}{2 \pi} \int_{0}^{2 \pi} \frac{1}{1+\operatorname{sen}^{2}(\tau)} d \tau=\frac{1}{2 \pi} \pi \sqrt{2}=\frac{\sqrt{2}}{2} \notin \mathbb{Z}
$$

Por outro lado, o item (II) do Teorema 2.6 implica que o operador

$$
\frac{\partial}{\partial t}+(1+i \operatorname{sen}(t))\left(\frac{\partial}{\partial x}+\frac{\partial}{\partial y}\right)
$$

é globalmente resolúvel.

Considerando agora o operador

$$
\frac{\partial}{\partial t}+\frac{\sqrt{2}}{2}(1+i \operatorname{sen}(x))\left(\frac{\partial}{\partial x}+\frac{\partial}{\partial y}\right)
$$

temos

$$
\frac{1}{2 \pi} \int_{0}^{2 \pi} \frac{\sqrt{2} / 2}{(\sqrt{2} / 2)^{2}\left[1+\operatorname{sen}^{2}(\tau)\right]} d \tau=\frac{1}{2 \pi} \frac{2}{\sqrt{2}} \pi \sqrt{2}=1 \in \mathbb{Z} ;
$$

logo o item (ii) do Teorema 4.1 implica que tal operador é globalmente resolúvel, se os subníveis

$$
\left\{x \in \mathbb{T}^{1} ; \int_{0}^{x} \frac{\operatorname{sen}(\tau)}{1+\operatorname{sen}^{2}(\tau)} d \tau<r\right\}(r \in \mathbb{R})
$$

são conexos. Como a função $\int_{0}^{x} \frac{\operatorname{sen}(\tau)}{1+\operatorname{sen}^{2}(\tau)} d \tau$ é estritamente crescente em $(0, \pi)$, estritamente decrescente em $(\pi, 2 \pi)$, possui um máximo em $x=\pi$ e mínimos em $x=0$ e $x=2 \pi$, segue que os subníveis acima são conexos. Portanto, o operador acima é globalmente resolúvel.

Em contraste, o item (II) do Teorema 2.6 implica que o operador

$$
\frac{\partial}{\partial t}+\frac{\sqrt{2}}{2}(1+i \operatorname{sen}(t))\left(\frac{\partial}{\partial x}+\frac{\partial}{\partial y}\right)
$$


não é globalmente resolúvel.

Observação 4.3. Note que, no Teorema 4.1, uma condição necessária para a resolubilidade global de L é que $\lambda$ não seja um número de Liouville. Veremos que tal condição também se verifica quando $c$ possui apenas um número finito de zeros.

Note também que, quando $c^{-1}(0)=\emptyset$, do Capítulo 2 segue que o operador $L$ é fortemente resolúvel se, e somente se:

- $b \equiv 0,(\alpha, 0, \lambda)$ satisfaz (DC4) e $\tau+j \alpha+k \lambda=0$ apenas para $j=k=\tau=0$; ou

- $b \not \equiv 0, \beta \neq 0, b$ não muda de sinal, $(\alpha, \beta, \lambda)$ satisfaz (DC4) e $\tau+j(\alpha+i \beta)+k \lambda=0$ apenas para $j=k=\tau=0$.

Como consequência do Teorema 4.1 obtemos o seguinte resultado:

Corolário 4.4. Se $b^{-1}(0)=\emptyset$, então o operador

$$
\frac{\partial}{\partial t}+i b(x)\left(\frac{\partial}{\partial x}+\lambda \frac{\partial}{\partial y}\right)
$$

é globalmente resolúvel se, e somente se, $\lambda$ não é um número de Liouville.

Passemos agora ao caso em que $c^{-1}(0) \neq \emptyset$. Afirmamos que $\mathrm{L}$ não é fortemente resolúvel. De fato, seja $x_{0} \in c^{-1}(0), \tilde{y} \in \mathbb{T}^{1}$ e $n$ um número inteiro não negativo menor do que a ordem de anulamento de $c$ em $x_{0}$. Cálculos diretos mostram que a distribuição $\delta^{(n)}\left(x-x_{0}\right) \otimes \delta(y-\tilde{y}) \otimes 1_{t}$ pertence ao núcleo de ${ }^{t} \mathrm{~L}$. Sendo assim, obtemos $\operatorname{dim} \operatorname{ker}^{t} \mathbf{L}=\infty$, ou seja, L não é fortemente resolúvel (Lema 1.1).

Procedendo como em $[\mathrm{BP}]$, veremos que $\mathrm{L}$ não é globalmente resolúvel quando a função $c$ possui pelo menos um zero de ordem infinita.

Lema 4.5. Suponha que $c^{-1}(0) \neq \mathbb{T}^{1}$ e que c possui um zero de ordem infinita em $\mathbb{T}^{1}$. Então o operador L (dado por (4.1)) não é globalmente resolúvel.

Demonstração: Mostraremos primeiro que a não resolubilidade global de $\mathrm{L}_{0} \doteq c(x) \frac{d}{d x}$ em $\mathbb{T}_{x}^{1}$ implica que $\mathrm{L}$ não é globalmente resolúvel em $\mathbb{T}^{3}$. De fato, suponha que existe $f \in\left(\operatorname{ker}^{t} \mathrm{~L}_{0}\right)^{\circ} \backslash \mathrm{L}_{0} \mathcal{C}^{\infty}\left(\mathbb{T}_{x}^{1}\right) \subset \mathcal{C}^{\infty}\left(\mathbb{T}_{x}^{1}\right)$. Para cada $\mu \in \operatorname{ker}^{t} \mathrm{~L} \subset \mathcal{D}^{\prime}\left(\mathbb{T}^{3}\right)$, utilizando a série 
parcial de Fourier de $\mu$ nas variáveis $(y, t)$, obtemos

$$
\begin{aligned}
\langle\mu, f\rangle & =\left\langle\sum_{(j, k) \in \mathbb{Z}^{2}} \hat{\mu}(\cdot, j, k) \otimes e^{i(j y+k t)}, f\right\rangle=\sum_{(j, k) \in \mathbb{Z}^{2}}\langle\hat{\mu}(\cdot, j, k), f\rangle\left\langle e^{i(j y+k t)}, 1\right\rangle \\
& =(2 \pi)^{2}\langle\hat{\mu}(\cdot, 0,0), f\rangle .
\end{aligned}
$$

Como $\mu \in \operatorname{ker}^{t} \mathrm{~L}$, temos $\hat{\mu}(\cdot, 0,0) \in \operatorname{ker}^{t} \mathrm{~L}_{0} ; \operatorname{logo}\langle\mu, f\rangle=(2 \pi)^{2}\langle\hat{\mu}(\cdot, 0,0), f\rangle=0$. Assim $f \in\left(\operatorname{ker}^{t} \mathrm{~L}\right)^{\circ}$. Por outro lado, se existir $u \in \mathcal{C}^{\infty}\left(\mathbb{T}^{3}\right)$ satisfazendo $\mathrm{L} u=f$, então teremos $\mathrm{L}_{0} \hat{u}(\cdot, 0,0)=f$, onde $\hat{u}(\cdot, 0,0) \in \mathcal{C}^{\infty}\left(\mathbb{T}_{x}^{1}\right)$; mas tal fato não pode ocorrer uma vez que $f \in \mathcal{C}^{\infty}\left(\mathbb{T}^{1}\right) \backslash \mathrm{L}_{0} \mathcal{C}^{\infty}\left(\mathbb{T}^{1}\right)$. Portanto, se $\mathrm{L}_{0}$ não é globalmente resolúvel, então $\mathrm{L}$ não é globalmente resolúvel.

Repetindo argumentos de $[\mathrm{BP}] \operatorname{com} c=a+i b$ no lugar de $a$, veremos que $\mathrm{L}_{0} \doteq c(x) \frac{d}{d x}$ não é globalmente resolúvel quando $c$ possui um zero de ordem infinita. De fato, note que $\operatorname{ker}^{t} \mathrm{~L}_{0}=\left\{\nu \in \mathcal{D}^{\prime}\left(\mathbb{T}^{1}\right) ; c \nu=0\right\}$, pois a única distribuição constante que pode ser dividida por uma função que é flat em algum ponto é a distribuição nula. Por hipótese $\emptyset \neq \mathcal{F} \doteq\left\{x \in \mathbb{T}^{1} ; c\right.$ é flat em $\left.x\right\} \neq \mathbb{T}^{1} ; \log \mathrm{\partial \mathcal {F }} \neq \emptyset$. Fixe $x_{1} \in \partial \mathcal{F} \neq \emptyset$. Considere $g(x)=1-\cos \left(x-x_{1}\right)$ e $f \doteq c / g \in \mathcal{C}^{\infty}\left(\mathbb{T}^{1}\right)$, a qual é flat em $x_{1}$. Para cada $\nu \in \operatorname{ker}^{t} \mathrm{~L}_{0}$ temos $g(f \nu)=c \nu=0, \operatorname{logo} f \nu=c_{0} \delta\left(x-x_{1}\right)+c_{1} \delta^{\prime}\left(x-x_{1}\right)$. A única distribuição do tipo $c_{0} \delta\left(x-x_{1}\right)+c_{1} \delta^{\prime}\left(x-x_{1}\right)$ que podemos dividir por uma função que é flat em $x_{1}$ é quando $c_{0}=c_{1}=0$, $\operatorname{logo} f \nu=0$ e, consequentemente, $\langle\nu, f\rangle=0$. Assim, $f \in\left(\operatorname{ker}^{t} \mathrm{~L}_{0}\right)^{\circ}$. Por fim, se existir $u \in \mathcal{C}^{\infty}\left(\mathbb{T}^{1}\right)$ tal que $\mathrm{L}_{0} u=f$, então $f=c u^{\prime}=f g u^{\prime}$. Tomando uma sequência $\left(y_{n}\right)$ tal que $y_{n} \rightarrow x_{1}$ e $c\left(y_{n}\right) \neq 0$, para todo $n \in \mathbb{N}$, obtemos $g\left(y_{n}\right) u^{\prime}\left(y_{n}\right)=1$, para todo $n \in \mathbb{N}$, logo $u^{\prime}\left(y_{n}\right) \rightarrow \infty$, o que é uma contradição.

Consideremos agora o caso em que $c$ só possui zeros de ordem finita e $c^{-1}(0) \neq \emptyset$. O próximo resultado diz que uma condição necessária para a resolubilidade global de L é que $\lambda$ não seja um número de Liouville.

Lema 4.6. Suponha que $c^{-1}(0)=\emptyset$ ou que c possui apenas zeros de ordem finita. Se $\lambda$ é um número de Liouville, então L (dado por (4.1)) não é globalmente resolúvel.

Demonstração: Note que se $\lambda$ é um número de Liouville, então $L_{\lambda}=\partial_{x}+\lambda \partial_{y}$ não é globalmente resolúvel em $\mathbb{T}^{2}$ (ver $\left.[\mathrm{BP}]\right)$, ou seja, existe $f \in\left(\operatorname{ker}^{t} \mathrm{~L}_{\lambda}\right)^{\circ} \subset \mathcal{C}^{\infty}\left(\mathbb{T}_{(x, y)}^{2}\right)$ tal que a equaçao $\mathrm{L}_{\lambda} v=f$ não tem solução $v$ em $\mathcal{C}^{\infty}\left(\mathbb{T}_{(x, y)}^{2}\right)$. Mostremos que $c f \in$ $\left(\operatorname{ker}^{t} \mathrm{~L}\right)^{\circ} \backslash \mathrm{LC} \mathcal{C}^{\infty}\left(\mathbb{T}^{3}\right)$, o que significa que $\mathrm{L}$ não é globalmente resolúvel, como queríamos. 
Para tanto, note que se $\mu \in \mathcal{D}^{\prime}\left(\mathbb{T}^{3}\right)$ e ${ }^{t} \mathrm{~L} \mu=0$, então ${ }^{t} \mathrm{~L}_{\lambda}\left(c \hat{\mu}_{0}\right)=0$, onde $\hat{\mu}_{k}$ denota os coeficientes da série parcial de Fourier de $\mu$ com relação à variável $t$. Desta forma, temos $\langle\mu, c f\rangle=2 \pi\left\langle\hat{\mu}_{0}, c f\right\rangle=2 \pi\left\langle c \hat{\mu}_{0}, f\right\rangle=0$, qualquer que seja $\mu \in \operatorname{ker}^{t} \mathrm{~L}$; logo $c f \in$ $\left(\operatorname{ker}^{t} \mathrm{~L}\right)^{\circ}$. Finalmente, se existir $u \in \mathcal{C}^{\infty}\left(\mathbb{T}^{3}\right)$ tal que $\mathrm{L} u=c f$, então $\hat{u}_{0}$ satisfaz $c(x)\left(\partial_{x}+\right.$ $\left.\lambda \partial_{y}\right) \hat{u}_{0}(x, y)=c(x) f(x, y)$ e como $c$ possui apenas um número finito de zeros (podendo ser nenhum zero), segue que a função $\hat{u}_{0}$ satisfaz $\mathrm{L}_{\lambda} \hat{u}_{0}=f \mathrm{em} \mathbb{T}_{(x, y)}^{2}$, o que é uma contradição. Portanto, $c f \in\left(\operatorname{ker}^{t} \mathrm{~L}\right)^{\circ} \backslash \mathrm{LC} \mathcal{C}^{\infty}\left(\mathbb{T}^{3}\right)$ e $\mathrm{L}$ não é globalmente resolúvel.

Para apresentarmos os resultados sobre a resolubilidade global de L, no caso em que $c$ só possui zeros de ordem finita e $c^{-1}(0) \neq \emptyset$, escrevemos

$$
c^{-1}(0) \doteq\left\{x_{1}<\cdots<x_{N}<x_{N+1} \doteq x_{1}+2 \pi\right\}
$$

Em cada $x_{\ell} \in c^{-1}(0)$, denote por $n_{\ell}$ e $m_{\ell}$ a ordem de anulamento (em $x_{\ell}$ ) das funções $a$ e $b$, respectivamente. Considere também os seguintes subconjuntos de $c^{-1}(0)$ :

$$
\mathrm{V}_{1} \doteq\left\{x_{\ell} \in c^{-1}(0) ; 2 \leqslant m_{\ell}<2 n_{\ell}-1, m_{\ell}<\infty, 1<n_{\ell} \leqslant \infty\right\}
$$

e

$$
\mathrm{V}_{2} \doteq\left\{x_{\ell} \in c^{-1}(0) ; 2 n_{\ell}-1<m_{\ell} \leqslant \infty, 1 \leqslant n_{\ell}<\infty\right\}
$$

O teorema a seguir é o principal resultado deste capítulo.

Teorema 4.7. Suponha que $\emptyset \neq c^{-1}(0)=\mathrm{V}_{1} \cup \mathrm{V}_{2}$ e que $\lambda$ não é um número de Liouville.

$O$ operador $\mathrm{L}$ (dado por (4.1)) é globalmente resolúvel se, para cada $\ell=1, \ldots, N$, ocorre uma das seguintes situações:

(I) $\left\{x_{\ell}, x_{\ell+1}\right\} \subset \mathrm{V}_{1}$ e existe $\eta \in\left(x_{\ell}, x_{\ell+1}\right)$ de modo que $b$ não muda de sinal nos intervalos $\left(x_{\ell}, \eta\right)$ e $\left(\eta, x_{\ell+1}\right)$;

(II) $\left\{x_{\ell}, x_{\ell+1}\right\} \subset \mathrm{V}_{2}, b$ não muda de sinal em $\left(x_{\ell}, x_{\ell+1}\right)$ e existe $\delta>0$ tal que $b \equiv 0$ nos intervalos $\left(x_{\ell}, x_{\ell}+\delta\right)$ e $\left(x_{\ell+1}-\delta, x_{\ell+1}\right)$;

(III) $x_{\ell} \in \mathrm{V}_{1}, x_{\ell+1} \in \mathrm{V}_{2}$, b não muda de sinal em $\left(x_{\ell}, x_{\ell+1}\right)$ e $b \equiv 0$ em $\left(x_{\ell+1}-\delta, x_{\ell+1}\right)$;

(IV) $x_{\ell} \in \mathrm{V}_{2}, x_{\ell+1} \in \mathrm{V}_{1}$, b não muda de sinal em $\left(x_{\ell}, x_{\ell+1}\right)$ e $b \equiv 0$ em $\left(x_{\ell}, x_{\ell}+\delta\right)$.

Por outro lado, L não é globalmente resolúvel quando existe $\ell \in\{1, \ldots, N\}$ tal que uma das seguintes situações se verifica: 
(V) $\left\{x_{\ell}, x_{\ell+1}\right\} \subset \mathrm{V}_{1}$ e $b$ muda de sinal mais de uma vez em $\left(x_{\ell}, x_{\ell+1}\right)$;

(VI) $x_{\ell} \in \mathrm{V}_{2}$ e $b$ muda de sinal em $\left(x_{\ell-1}, x_{\ell}\right)$ ou em $\left(x_{\ell}, x_{\ell+1}\right)\left(\right.$ onde $\left.x_{0} \doteq x_{N}-2 \pi\right)$.

Os resultados abaixo são casos particulares do Teorema 4.7, onde separamos o caso em que ocorre apenas zeros do tipo $V_{1}$ e o caso em que ocorre apenas zeros do tipo $V_{2}$. As demonstrações de tais resultados e a demonstração do Teorema 4.7 serão apresentadas nas próximas seções.

Teorema 4.8. Suponha que $\emptyset \neq c^{-1}(0)=\mathrm{V}_{1}$ e que $\lambda$ não é um número de Liouville. Neste caso, o operador $\mathrm{L}$ (dado por (4.1)) é globalmente resolúvel se, e somente se, b não muda de sinal ou b muda de sinal no máximo uma vez em cada intervalo $\left(x_{\ell}, x_{\ell+1}\right)$ (ou seja, existe $\eta \in\left(x_{\ell}, x_{\ell+1}\right)$ tal que $b$ não muda de sinal nos intervalos $\left(x_{\ell}, \eta\right)$ e $\left.\left(\eta, x_{\ell+1}\right)\right)$.

Corolário 4.9. Suponha que $\lambda$ não é um número de Liouville. Suponha ainda que $b^{-1}(0) \neq \emptyset$ e que cada zero de $b$ é de ordem finita $\geqslant 2$. Nestas condições, o operador

$$
\frac{\partial}{\partial t}+i b(x)\left(\frac{\partial}{\partial x}+\lambda \frac{\partial}{\partial y}\right)
$$

é globalmente resolúvel.

Teorema 4.10. Suponha que $\lambda$ não é um número de Liouville e que $\emptyset \neq c^{-1}(0)=\mathrm{V}_{2}$. Se existe $\ell \in\{1, \ldots, N\}$ tal que $b$ muda de sinal em $\left(x_{\ell}, x_{\ell+1}\right)$, então $\mathrm{L}$ não é globalmente resolúvel. Se b não muda de sinal em cada intervalo $\left(x_{\ell}, x_{\ell+1}\right)$ e se existe $\delta>0$ tal que $b \equiv 0$ em $\left(x_{\ell}, x_{\ell}+\delta\right) \cup\left(x_{\ell+1}-\delta, x_{\ell+1}\right)$, então L é globalmente resolúvel.

Dos teoremas 4.1 e 4.10 segue o seguinte resultado sobre operadores reais:

Corolário 4.11. O operador

$$
\frac{\partial}{\partial t}+a(x)\left(\frac{\partial}{\partial x}+\lambda \frac{\partial}{\partial y}\right)
$$

é globalmente resolúvel se, e somente se, ocorre uma das seguintes situações:

(i) $a^{-1}(0)=\mathbb{T}^{1}$;

(ii) $a^{-1}(0)=\emptyset e(\alpha, 0, \lambda)$ satisfaz (DC4), onde $\alpha=(2 \pi)^{-1} \int_{0}^{2 \pi} a^{-1}(x) d x$.

(iii) $a^{-1}(0) \neq \emptyset$, a se anula apenas de ordem finita e $\lambda$ é não-Liouville. 
Observação 4.12. Quando a é uma função constante, o item (ii) do Corolário 4.11 e o item (I) do Teorema 2.6 fornecem duas condições equivalentes para a resolubilidade global do operador em questão.

Como consequência da demonstração do Teorema 4.8, também seguirá o seguinte resultado sobre campos vetoriais no toro $\mathbb{T}^{2}$, os quais podem não satisfazer a condição $(\mathcal{P})$.

Corolário 4.13. Considere o operador $\partial_{t}+(a(x)+i b(x)) \partial_{x}$ em $\mathbb{T}^{2}$. Suponha que $c=a+i b$ se anule apenas de ordem finita e que $\emptyset \neq c^{-1}(0)=\mathrm{V}_{1} \cup \mathrm{V}_{2}$. Tal operador é globalmente resolúvel se para todo $\ell=1, \ldots, N$ ocorrer uma das condições de (I) a (IV) dadas no Teorema 4.7. Por outro lado, o operador não é globalmente resolúvel se para algum $\ell$ ocorrer (V) ou (VI). Em particular, quando $c^{-1}(0)=\mathrm{V}_{1}$, tal operador é globalmente resolúvel se, e somente se, $b$ muda de sinal no máximo uma vez em cada intervalo $\left(x_{\ell}, x_{\ell+1}\right)$.

Quando $b$ não muda de sinal em cada intervalo $\left(x_{\ell}, x_{\ell+1}\right)$, o Corolário 4.13 está de acordo com os resultados de [BD1].

Antes de iniciarmos as demonstrações, vamos apresentar dois exemplos.

Exemplo 4.14. O operador

$$
\frac{\partial}{\partial t}+\left(\operatorname{sen}^{3}(x)+i \operatorname{sen}^{3}(2 x)\right)\left(\frac{\partial}{\partial x}+\frac{\partial}{\partial y}\right)
$$

é globalmente resolúvel. Com efeito, a função $c(x)=\operatorname{sen}^{3}(x)+i \operatorname{sen}^{3}(2 x)$ satisfaz $c^{-1}(0)=\{0, \pi\}$ e as funções $a(x)=\operatorname{sen}^{3}(x)$ e $b(x)=\operatorname{sen}^{3}(2 x)$ se anulam de ordem $3 \mathrm{em}$ 0 e $\pi$. Assim, estamos nas condições do Teorema 4.8. Além disso, nos intervalos $(0, \pi)$ e $(\pi, 2 \pi)$, a função $b(x)=\operatorname{sen}^{3}(2 x)$ muda de sinal apenas uma vez; logo o operador acima é globalmente resolúvel.

Por outro lado, é interessante lembrar que o operador

$$
\frac{\partial}{\partial t}+\left(\operatorname{sen}^{3}(t)+i \operatorname{sen}^{3}(2 t)\right)\left(\frac{\partial}{\partial x}+\frac{\partial}{\partial y}\right)
$$

não é globalmente resolúvel, conforme Exemplo 2.15. 
Exemplo 4.15. Considere o operador

$$
\frac{\partial}{\partial t}+\left(\operatorname{sen}(x)+i \operatorname{sen}^{3}(2 x)\right)\left(\frac{\partial}{\partial x}+\frac{\partial}{\partial y}\right) .
$$

Para $c(x)=\operatorname{sen}(x)+i \operatorname{sen}^{3}(2 x), \operatorname{temos} c^{-1}(0)=\{0, \pi\}$. Como $a(x)=\operatorname{sen}(x)$ se anula de ordem 1 em $x=0$ e $x=\pi$ e como $b(x)=\operatorname{sen}^{3}(2 x)$ se anula de ordem 3 nesses pontos, estamos nas condições do Teorema 4.10. Além disso, como $\operatorname{sen}^{3}(2 x)$ muda de sinal em $(0, \pi)$, segue que o operador acima não é globalmente resolúvel.

Olhando o que ocorre quando o coeficiente depende da variável $t$, obtemos o campo

$$
\frac{\partial}{\partial t}+\left(\operatorname{sen}(t)+i \operatorname{sen}^{3}(2 t)\right)\left(\frac{\partial}{\partial x}+\frac{\partial}{\partial y}\right)
$$

o qual também não é globalmente resolúvel pelo Teorema 2.6; para encontrar um subnível desconexo, basta proceder como no Exemplo 2.15.

Por outro lado, o operador

$$
\frac{\partial}{\partial t}+\left(1-\cos (x)+i \operatorname{sen}^{5}(x)\right)\left(\frac{\partial}{\partial x}+\frac{\partial}{\partial y}\right)
$$

está nas condições do Teorema 4.10 e não é globalmente resolúvel. Enquanto que

$$
\frac{\partial}{\partial t}+\left(1-\cos (t)+i \operatorname{sen}^{5}(t)\right)\left(\frac{\partial}{\partial x}+\frac{\partial}{\partial y}\right)
$$

é globalmente resolúvel, pelo Teorema 2.6.

Organizaremos a demonstração do Teorema 4.7 separando-a em partes. Demonstraremos primeiro os teoremas 4.8 e 4.10, os quais são casos particulares do Teorema 4.7 .

O próximo resultado nos auxiliará na demonstração de que L é globalmente resolúvel, quando estiverem satisfeitas as condições apresentadas no Teorema 4.7. Tal resultado é conhecido como "redução módulo flat"(ver [BP], [BD1] e [BCP]).

Lema 4.16. Suponha que $c^{-1}(0) \neq \emptyset$ e que c possui apenas anulamentos de ordem finita. Além disso, se $m_{\ell} \geqslant 2$ ou $m_{\ell}=n_{\ell}=1$, para todo $\ell=1, \ldots, N$, então dada $f \in\left(\operatorname{ker}^{t} \mathrm{~L}\right)^{\circ}$, existe $u \in \mathcal{C}^{\infty}\left(\mathbb{T}^{3}\right)$ tal que $\mathrm{L} u-f$ é flat em $c^{-1}(0) \times \mathbb{T}^{2}$. 
Demonstração: Como em [BP], Lema 2.1, basta resolvermos em uma vizinhança de $\left\{x_{0}\right\} \times \mathbb{T}^{2}$, para cada $x_{0} \in c^{-1}(0)$. Sejam $n$ e $m$ as ordens de anulamento de $a$ e $b$, respectivamente, em $x_{0}$.

Utilizando a expansão formal em série de Taylor em uma vizinhança de $x_{0}$, podemos escrever

$$
\begin{gathered}
a \simeq a_{n}\left(x-x_{0}\right)^{n}+a_{n+1}\left(x-x_{0}\right)^{n+1}+\cdots, \\
b \simeq b_{m}\left(x-x_{0}\right)^{m}+b_{m+1}\left(x-x_{0}\right)^{m+1}+\cdots, \\
u \simeq u_{0}(y, t)+u_{1}(y, t)\left(x-x_{0}\right)+u_{2}(y, t)\left(x-x_{0}\right)^{2}+\cdots
\end{gathered}
$$

e

$$
f \simeq f_{0}(y, t)+f_{1}(y, t)\left(x-x_{0}\right)+f_{2}(y, t)\left(x-x_{0}\right)^{2}+\cdots
$$

Note que $a_{n} \neq 0$ e $b_{m} \neq 0$.

O próximo passo será determinar uma sequência $\left(u_{k}\right)_{k \geqslant 0} \subset \mathcal{C}^{\infty}\left(\mathbb{T}^{2}\right)$ de modo que $\mathrm{L} u-f \simeq 0$. Para tanto, vamos considerar os seguintes casos: $m=n=1,1=n<m$, $1<n=m, 1<n<m$ e $1<m<n$.

Caso 1: $m=n=1$.

Neste caso, temos $\mathrm{L} u-f \simeq 0$ se, e somente se,

$$
\partial_{t} u_{0}=f_{0}
$$

e para $k \geqslant 1$

$$
\partial_{t} u_{k}+\sum_{j=1}^{k}\left(a_{j}+i b_{j}\right)\left[(k-j+1) u_{k-j+1}+\lambda \partial_{y} u_{k-j}\right]=f_{k}
$$

Para $y^{\prime} \in \mathbb{T}^{1}$, a distribuição $\delta\left(x-x_{0}\right) \otimes \delta\left(y-y^{\prime}\right) \otimes 1_{t}$ pertence a $\operatorname{ker}^{t} \mathbf{L}$ (pois $c(x)$ se anula de ordem 1 em $x_{0}$ ). Assim,

$$
0=\int_{0}^{2 \pi} f\left(x_{0}, y^{\prime}, t\right) d t=\int_{0}^{2 \pi} f_{0}\left(y^{\prime}, t\right) d t
$$

para todo $y^{\prime} \in \mathbb{T}^{1}$. Portanto, a equação (4.3) possui infinitas soluções, as quais possuem a forma geral

$$
\int_{0}^{t} f_{0}(y, s) d s+C_{0}(y)
$$


onde $C_{0} \in \mathcal{C}^{\infty}\left(\mathbb{T}^{1}\right)$.

Vamos agora olhar para as equações (4.4), as quais se reescrevem na forma

$$
\partial_{t} u_{1}+\left(a_{1}+i b_{1}\right) u_{1}=f_{1}-\left(a_{1}+i b_{1}\right) \lambda \partial_{y} u_{0}
$$

e para $k \geqslant 2$,

$\partial_{t} u_{k}+\left(a_{1}+i b_{1}\right) k u_{k}=f_{k}-\left(a_{1}+i b_{1}\right) \lambda \partial_{y} u_{k-1}-\sum_{j=2}^{k}\left(a_{j}+i b_{j}\right)\left[(k-j+1) u_{k-j+1}+\lambda \partial_{y} u_{k-j}\right]$

Como $a_{1} \neq 0$, utilizando série de Fourier nas variáveis $(y, t)$ e procedendo como na demonstração da Proposição 2.17, podemos determinar sucessivamente a única solução de (4.4), para todo $k \geqslant 1$, obtendo uma sequência $\left(u_{k}\right)_{k \geqslant 0}$ que satisfaz (4.3) e (4.4).

Caso 2: $1=n<m$.

Quando $1=n<m$, temos $\mathrm{L} u-f \simeq 0$ se, e somente se,

$$
\begin{gathered}
\partial_{t} u_{0}=f_{0}, \\
\partial_{t} u_{k}+\sum_{j=1}^{k} a_{j}\left[(k-j+1) u_{k-j+1}+\lambda \partial_{y} u_{k-j}\right]=f_{k}, \quad 1 \leqslant k<m,
\end{gathered}
$$

e para $k \geqslant m$

$\partial_{t} u_{k}+\sum_{j=1}^{m-1} a_{j}\left[(k-j+1) u_{k-j+1}+\lambda \partial_{y} u_{k-j}\right]+\sum_{j=m}^{k}\left(a_{j}+i b_{j}\right)\left[(k-j+1) u_{k-j+1}+\lambda \partial_{y} u_{k-j}\right]=f_{k}$.

Como no caso 1, a equação (4.5) possui infinitas soluções, as quais possuem a forma geral

$$
\int_{0}^{t} f_{0}(y, s) d s+C_{0}(y), \quad \text { onde } C_{0} \in \mathcal{C}^{\infty}\left(\mathbb{T}^{1}\right)
$$

Para $1 \leqslant k<m$, como $a_{1} \neq 0$, podemos determinar sucessivamente a única solução de cada equação (4.6), basta proceder conforme o que foi feito no caso 1 para as equações do tipo (4.4). Analogamente, para $k \geqslant m(\geqslant 2), a_{1} \neq 0$ implica que podemos determinar sucessivamente a única solução de cada equação (4.7).

Portanto, obtemos uma sequência $\left(u_{k}\right)_{k \geqslant 0}$ que satisfaz (4.5), (4.6) e (4.7).

Caso 3: $1<n=m$. 
Quando tal relação ocorrer, teremos $L u-f \simeq 0$ se, e somente se,

$$
\partial_{t} u_{j}=f_{j}, \quad j=0, \ldots, n-1
$$

e para $k \geqslant n$

$$
\partial_{t} u_{k}+\sum_{j=n}^{k}\left(a_{j}+i b_{j}\right)\left[(k-j+1) u_{k-j+1}+\lambda \partial_{y} u_{k-j}\right]=f_{k}
$$

O anulamento de ordem $n$ de $c(x)$ em $x_{0}$ implica que, para $y^{\prime} \in \mathbb{T}^{1}$, a distribuição $\delta^{(\ell)}\left(x-x_{0}\right) \otimes \delta\left(y-y^{\prime}\right) \otimes 1_{t}(\ell=1, \ldots, n-1)$ pertence a ker ${ }^{t} \mathrm{~L}$. Assim,

$$
0=\int_{0}^{2 \pi} \partial_{x}^{\ell} f\left(x_{0}, y^{\prime}, t\right) d t=(\ell !) \int_{0}^{2 \pi} f_{\ell}\left(y^{\prime}, t\right) d t
$$

para todo $y^{\prime} \in \mathbb{T}^{1}$ e, portanto, cada equação (4.8) possui infinitas soluções, as quais possuem a forma geral

$$
\int_{0}^{t} f_{j}(y, s) d s+C_{j}(y)
$$

onde $C_{j}$ é uma função arbitrária em $\mathcal{C}^{\infty}\left(\mathbb{T}^{1}\right)$, a qual será fixada de maneira apropriada posteriormente.

Para $k=n$, a equação (4.9) se torna

$$
\partial_{t} u_{n}+\left(a_{n}+i b_{n}\right)\left[u_{1}+\lambda \partial_{y} u_{0}\right]=f_{n}
$$

e como $n>1$, tal equação tem solução se, e somente se,

$$
\int_{0}^{2 \pi}\left(f_{n}-\left(a_{n}+i b_{n}\right)\left[u_{1}+\lambda \partial_{y} u_{0}\right]\right)(y, t) d t=0
$$

para todo $y \in \mathbb{T}^{1}$. Sendo assim, reescolhendo a solução $u_{1}$, ou seja, fixando $C_{1}$ tal que

$$
C_{1}(y)=\left(2 \pi\left(a_{n}+i b_{n}\right)\right)^{-1} \int_{0}^{2 \pi}\left(f_{n}(y, t)-\left(a_{n}+i b_{n}\right)\left[\int_{0}^{t} f_{1}(y, s) d s+\lambda \partial_{y} u_{0}(y, t)\right]\right) d t
$$

segue que podemos resolver (4.10). 
A solução geral de (4.10) é dada por

$$
\int_{0}^{t}\left(f_{n}-\left(a_{n}+i b_{n}\right)\left[u_{1}+\lambda \partial_{y} u_{0}\right]\right)(y, s) d s+C_{n}(y)
$$

onde, a priori, $C_{n}$ é uma função arbitrária em $\mathcal{C}^{\infty}\left(\mathbb{T}^{1}\right)$. Repetindo esse processo recursivo e fixando de maneira apropriada as funções $C_{1}, C_{2}, \ldots$, obtemos uma sequência $\left(u_{k}\right)_{k \geqslant 0}$ que satisfaz (4.8) e (4.9).

Caso 4: $1<n<m$.

Neste caso, temos $L u-f \simeq 0$ se, e somente se,

$$
\begin{gathered}
\partial_{t} u_{j}=f_{j}, \quad j=0, \ldots, n-1, \\
\partial_{t} u_{k}+\sum_{j=n}^{k} a_{j}\left[(k-j+1) u_{k-j+1}+\lambda \partial_{y} u_{k-j}\right]=f_{k}, \quad n \leqslant k<m,
\end{gathered}
$$

e para $k \geqslant m$

$\partial_{t} u_{k}+\sum_{j=n}^{m-1} a_{j}\left[(k-j+1) u_{k-j+1}+\lambda \partial_{y} u_{k-j}\right]+\sum_{j=m}^{k}\left(a_{j}+i b_{j}\right)\left[(k-j+1) u_{k-j+1}+\lambda \partial_{y} u_{k-j}\right]=f_{k}$.

Como antes, o anulamento de ordem $n$ de $c(x)$ em $x_{0}$ implica que cada equação (4.11) possui infinitas soluções, as quais possuem a forma geral

$$
\int_{0}^{t} f_{j}(y, s) d s+C_{j}(y)
$$

onde $C_{j}$ é uma função arbitrária em $\mathcal{C}^{\infty}\left(\mathbb{T}^{1}\right)$, a qual será fixada de maneira apropriada posteriormente.

Para $k=n$, a equação (4.12) se torna

$$
\partial_{t} u_{n}+a_{n}\left[u_{1}+\lambda \partial_{y} u_{0}\right]=f_{n}
$$

e como $n>1$, tal equação tem solução se, e somente se,

$$
\int_{0}^{2 \pi}\left(f_{n}-a_{n}\left[u_{1}+\lambda \partial_{y} u_{0}\right]\right)(y, t) d t=0
$$


para todo $y \in \mathbb{T}^{1}$. Tal condição está satisfeita se reescolhermos a solução $u_{1}$, ou seja, se fixarmos $C_{1}$ tal que

$$
C_{1}(y)=\left(2 \pi a_{n}\right)^{-1} \int_{0}^{2 \pi}\left(f_{n}(y, t)-a_{n}\left[\int_{0}^{t} f_{1}(y, s) d s+\lambda \partial_{y} u_{0}(y, t)\right]\right) d t
$$

A solução geral de (4.14) é dada por

$$
\int_{0}^{t}\left(f_{n}-a_{n}\left[u_{1}+\lambda \partial_{y} u_{0}\right]\right)(y, s) d s+C_{n}(y)
$$

onde $C_{n}$ é uma função arbitrária em $\mathcal{C}^{\infty}\left(\mathbb{T}^{1}\right)$, a qual será fixada de maneira apropriada posteriormente.

Repetindo os argumentos acima, obtemos sucessivamente soluções de (4.12), para $k=n, \ldots, m-1$, de modo que, para qualquer $C_{k} \in \mathcal{C}^{\infty}\left(\mathbb{T}^{1}\right), u_{k}(y, t)+C_{k}(y)$ é ainda solução da respectiva equação (4.12).

Até aqui, obtemos sucessivamente $u_{0}, \ldots, u_{n}, \ldots, u_{m-1}$, soluções de (4.11) e (4.12), onde foi necessário fixar $C_{1}, \ldots, C_{m-n}$ apropriadamente.

Para $k=m$, a equação (4.13) se torna

$$
\partial_{t} u_{m}+\sum_{j=n}^{m-1} a_{j}\left[(m-j+1) u_{m-j+1}+\lambda \partial_{y} u_{m-j}\right]+\left(a_{m}+i b_{m}\right)\left[u_{1}+\lambda \partial_{y} u_{0}\right]=f_{m}
$$

Como $m>n>1$ e $a_{n} \neq 0$, podemos proceder como anteriormente, quando fixamos as soluções das equações (4.12), para obter soluções $u_{m}(y, t)+C_{m}(y)$ de $(4.15)$, onde foi necessário fixar a função $C_{m-n+1}$. Do mesmo modo, obtemos sucessivamente soluções $u_{k}(y, t)+C_{k}(y)(k \geqslant m)$ de cada equação (4.13), fixando as funções $C_{m-n+1}, C_{m-n+2}, \ldots$ de maneira apropriada.

Daí, obtemos uma sequência $\left(u_{k}\right)_{k \geqslant 0}$ que satisfaz (4.11), (4.12) e (4.13).

Caso 5: $2 \leqslant m<n$.

Neste último caso, temos $L u-f \simeq 0$ se, e somente se,

$$
\begin{gathered}
\partial_{t} u_{j}=f_{j}, \quad j=0, \ldots, m-1, \\
\partial_{t} u_{k}+\sum_{j=m}^{k} i b_{j}\left[(k-j+1) u_{k-j+1}+\lambda \partial_{y} u_{k-j}\right]=f_{k}, \quad m \leqslant k<n,
\end{gathered}
$$


e para $k \geqslant n$

$\partial_{t} u_{k}+\sum_{j=m}^{n-1} i b_{j}\left[(k-j+1) u_{k-j+1}+\lambda \partial_{y} u_{k-j}\right]+\sum_{j=n}^{k}\left(a_{j}+i b_{j}\right)\left[(k-j+1) u_{k-j+1}+\lambda \partial_{y} u_{k-j}\right]=f_{k}$.

Repetindo os argumentos do caso 4 , obtemos uma sequência $\left(u_{k}\right)_{k \geqslant 0} \subset \mathcal{C}^{\infty}\left(\mathbb{T}^{2}\right)$ que satisfaz (4.16), (4.17) e (4.18).

Por fim, como em $[\mathrm{BP}]$, uma aplicação do Teorema de Borel (ver também [G]) nos fornece uma função suave e com suporte compacto em uma vizinhança de $\left\{x_{0}\right\} \times \mathbb{T}^{2}, u$, tal que $(k !)^{-1} \partial_{x}^{k} u\left(x_{0}, y, t\right)=u_{k}$, para todo $k \geqslant 0$. Consequentemente, $\mathrm{L} u=f$.

O que conclui a demonstração do Lema 4.16.

\section{I Demonstração do Teorema 4.8}

Organizaremos a demonstração do Teorema 4.8 dividindo-a em proposições.

Lembre-se que estamos considerando o campo (dado por (4.1))

$$
\mathbf{L}=\frac{\partial}{\partial t}+(a(x)+i b(x))\left(\frac{\partial}{\partial x}+\lambda \frac{\partial}{\partial y}\right)
$$

e que estamos sob as seguintes condições: $\lambda$ não é um número de Liouville, $c$ possui apenas zeros de ordem finita e

$$
\begin{aligned}
\emptyset \neq c^{-1}(0) & =\left\{x_{1}<\ldots<x_{N}<x_{N+1} \doteq x_{1}+2 \pi\right\} \\
& =\mathrm{V}_{1}=\left\{x_{\ell} \in c^{-1}(0) ; 2 \leqslant m_{\ell}<2 n_{\ell}-1, m_{\ell}<\infty, 1<n_{\ell} \leqslant \infty\right\}
\end{aligned}
$$

Verificaremos primeiro que a condição de que $b$ muda de sinal no máximo uma vez em cada intervalo $\left(x_{\ell}, x_{\ell+1}\right)$ é suficiente para que o operador $\mathrm{L}$ seja globalmente resolúvel. Isso será feito com as proposições 4.17 e 4.18 .

Dada $f \in\left(\operatorname{ker}^{t} \mathrm{~L}\right)^{\circ}$, precisaremos mostrar que $f \in \mathrm{L} \mathcal{C}^{\infty}\left(\mathbb{T}^{3}\right)$. Do Lema 4.16 segue que existe $v \in \mathcal{C}^{\infty}\left(\mathbb{T}^{3}\right)$ tal que $L v-f$ é flat em $c^{-1}(0) \times \mathbb{T}^{2}$. Como $L v-f \in\left(\operatorname{ker}^{t} \mathrm{~L}\right)^{\circ}$, se pudermos encontrar $u \in \mathcal{C}^{\infty}\left(\mathbb{T}^{3}\right)$ tal que $\mathrm{L} u=\mathrm{L} v-f$, então teremos $f=\mathrm{L}(v-u) \in$ $\mathrm{LC}^{\infty}\left(\mathbb{T}^{3}\right)$. Portanto, do Lema 4.16 segue que podemos supor que o segundo membro $(f)$ é flat em $c^{-1}(0) \times \mathbb{T}^{2}$. 
Proposição 4.17. Suponha que c possui apenas anulamentos de ordem finita e que $\emptyset \neq c^{-1}(0)=\mathrm{V}_{1}$, como em (4.19). Suponha ainda que $\lambda$ não é um número de Liouville $e$ que $b$ não muda de sinal em cada intervalo $\left(x_{\ell}, x_{\ell+1}\right), \ell=1, \ldots, N$. Nestas condições, para cada $f \in\left(\operatorname{ker}^{t} \mathrm{~L}\right)^{\circ}$ que é flat em $c^{-1}(0) \times \mathbb{T}^{2}$, existe $u \in \mathcal{C}^{\infty}\left(\mathbb{T}^{3}\right)$ tal que $\mathrm{L} u=f$.

Demonstração: Dada $f \in\left(\operatorname{ker}^{t} \mathrm{~L}\right)^{\circ}, f$ flat em $c^{-1}(0) \times \mathbb{T}^{2}$, vamos procurar $u \in \mathcal{C}^{\infty}\left(\mathbb{T}^{3}\right)$ tal que $\mathbf{L} u=f$. Utilizando série parcial de Fourier nas variáveis $(y, t)$, segue que $\mathbf{L} u=f$ se, e somente se,

$$
c(x) \partial_{x} \hat{u}(x, j, k)+i(k+c(x) \lambda j) \hat{u}(x, j, k)=\hat{f}(x, j, k),
$$

para todo $x \in \mathbb{T}^{1}$ e $(j, k) \in \mathbb{Z}^{2}$.

O problema agora se resume a encontrar sequências $\hat{u}(\cdot, j, 0)_{j \in \mathbb{Z}}$ e $\hat{u}(\cdot, j, k)(j \in \mathbb{Z}$ e $k \neq 0$ ), que são soluções de (4.20) e que decaem rapidamente. Vamos olhar separadamente os casos $k=0, j \in \mathbb{Z}$ e $k \neq 0, j \in \mathbb{Z}$.

\section{Caso 1: $k=0, j \in \mathbb{Z}$}

Quando $k=0$, a equação (4.20) se reduz a

$$
\partial_{x} \hat{u}(x, j, 0)+i \lambda j \hat{u}(x, j, 0)=\hat{f}(x, j, 0) / c(x)
$$

para todo $x \in \mathbb{T}^{1}$ e $j \in \mathbb{Z}$. Neste caso, a busca de uma sequência de soluções $\hat{u}(\cdot, j, 0)$ (em $\mathcal{C}^{\infty}\left(\mathbb{T}^{1}\right)$ ) que decaia rapidamente fica semelhante ao que foi feito no caso tubo (Capítulo $2)$.

Para $j=0$, precisamos resolver $\partial_{x} \hat{u}(x, 0,0)=\hat{f}(x, 0,0) / c(x)$. Como $c$ se anula apenas de ordem finita, existe $(1 / c) \in \mathcal{D}^{\prime}\left(\mathbb{T}^{1}\right)$ tal que $c(1 / c)=1$ (tal distribuição pode não ser única). Cálculos diretos mostram que $(1 / c)(x) \otimes 1_{y} \otimes 1_{t}$ pertence a $\operatorname{ker}^{t} \mathrm{~L}$, $\operatorname{logo}$ $\int_{0}^{2 \pi} \hat{f}(x, 0,0) / c(x) d x=(2 \pi)^{-2} \int_{\mathbb{T}^{3}} f / c=(2 \pi)^{-2}\left\langle(1 / c)(x) \otimes 1_{y} \otimes 1_{t}, f\right\rangle=0$. Sendo assim, podemos fixar a solução

$$
\hat{u}(x, 0,0)=\int_{0}^{x} \hat{f}\left(x^{\prime}, 0,0\right) / c\left(x^{\prime}\right) d x^{\prime} .
$$

Com o objetivo de resolver (4.21) para $j \neq 0$, suponha primeiro que $\lambda \in \mathbb{R} \backslash \mathbb{Q}$ e que $\lambda$ não é um número de Liouville. Segue do Lema 1.6 que cada equação (4.21) possui 
uma única solução, a qual podemos escrever na forma

$$
\hat{u}(x, j, 0) \doteq\left(1-e^{-2 \pi i \lambda j}\right)^{-1} \int_{0}^{2 \pi} \exp \left\{-i \lambda j x^{\prime}\right\} \hat{f}\left(x-x^{\prime}, j, 0\right) / c\left(x-x^{\prime}\right) d x^{\prime}
$$

Como $\lambda$ é um irracional não-Liouville, existem constantes $C>0$ e $\gamma>0$ tais que $|\tau+j \lambda|>C(|j|+|\tau|)^{-\gamma}$, para todo $(j, k) \in \mathbb{Z}^{2} \backslash\{0\}$; logo vale (DC2) e pelo Lema 2.23 vale (DC3), ou seja, existem constantes $C^{\prime}>0$ e $\gamma^{\prime}>0$ tais que $\left|1-e^{-2 \pi i \lambda j}\right| \geqslant C^{\prime}|j|^{-\gamma^{\prime}}$, para todo $j \in \mathbb{Z} \backslash\{0\}$. Além disso, $(\hat{f}(\cdot, j, k) / c(\cdot))_{(j, k) \in \mathbb{Z}^{2}}$ decai rapidamente, logo a sequência $(\hat{u}(\cdot, j, 0))_{j \in \mathbb{Z}}$ também decai rapidamente.

Suponha agora que $\lambda=p / q \in \mathbb{Q}$, onde $p \in \mathbb{Z}$ e $q \in \mathbb{N}((p, q)=1$ se $\lambda \neq 0$ ou $p=0$ e $q=1$ se $\lambda=0$ ). Para os índices $j \notin q \mathbb{Z}$ (o qual é vazio se $q=1$ ), a equação (4.21) possui uma única solução, a qual também é dada pela fórmula (4.22). Além disso, tal sequência de soluções também decai rapidamente, pois $(\hat{f}(\cdot, j, k) / c(\cdot))_{(j, k) \in \mathbb{Z}^{2}}$ decai rapidamente e $\left|1-e^{-2 \pi i \lambda j}\right|^{-1}(j \in \mathbb{Z} \backslash q \mathbb{Z})$ é limitada (já que $|\tau+\lambda j|=|\tau+p j / q| \geqslant 1 / q$, quaisquer que sejam $\tau \in \mathbb{Z}$ e $j \in \mathbb{Z} \backslash q \mathbb{Z}$ ). Quando $j \in q \mathbb{Z} \backslash\{0\}$, a equação (4.21) possui infinitas soluções, pois $\int_{0}^{2 \pi} \hat{f}(x, j, 0) / c(x) e^{i \lambda j x} d x=0$, uma vez que $\left[e^{i \lambda j x} / c(x)\right] \otimes e^{-i j y} \otimes 1_{t} \in \operatorname{ker}^{t} \mathbf{L}$. Assim, para cada $j \in q \mathbb{Z} \backslash\{0\}$, podemos fixar a solução

$$
\hat{u}(x, j, 0) \doteq \int_{0}^{x} \exp \left\{i \lambda j\left(x^{\prime}-x\right)\right\} \hat{f}\left(x^{\prime}, j, 0\right) / c\left(x^{\prime}\right) d x^{\prime}
$$

Novamente, o decaimento rápido de $(\hat{f}(\cdot, j, k) / c(\cdot))_{(j, k) \in \mathbb{Z}^{2}}$ implica que $(\hat{u}(\cdot, j, 0))_{j \in q \mathbb{Z}}$ decai rapidamente.

Portanto, obtemos uma sequência de soluções de $(4.21), \hat{u}(\cdot, j, 0) \in \mathcal{C}^{\infty}\left(\mathbb{T}^{1}\right)(j \in \mathbb{Z})$, a qual decai rapidamente.

\section{Caso 2: $k \neq 0, j \in \mathbb{Z}$}

Neste caso, mostraremos inicialmente que (4.20) tem uma solução suave em cada intervalo $\left[x_{\ell}, x_{\ell+1}\right]$, a qual é flat em $\left\{x_{\ell}, x_{\ell+1}\right\}$, onde $\ell=1, \ldots, N$. Note que, em $\left(x_{\ell}, x_{\ell+1}\right)$ a equação (4.20) se torna

$$
\partial_{x} \hat{u}(x, j, k)+i(k / c(x)+\lambda j) \hat{u}(x, j, k)=\hat{f}(x, j, k) / c(x),
$$

para todo $(j, k) \in \mathbb{Z} \times(\mathbb{Z} \backslash\{0\})$.

Por hipótese, $b$ não muda de sinal em $\left(x_{\ell}, x_{\ell+1}\right)$. 
Suponha primeiro que $b \geqslant 0$ em $\left(x_{\ell}, x_{\ell+1}\right)$. Se $k>0$ e se $x \in\left(x_{\ell}, x_{\ell+1}\right)$ está fixado, a função

$$
\left(x_{\ell}, x\right) \ni x^{\prime} \mapsto \exp \left\{-k \int_{x^{\prime}}^{x} \frac{b+i a}{a^{2}+b^{2}}(s) d s+i \lambda j\left(x^{\prime}-x\right)\right\} \hat{f}\left(x^{\prime}, j, k\right) / c\left(x^{\prime}\right)
$$

está bem definida e pertence a $\mathcal{C}^{\infty}\left(\left(x_{\ell}, x\right)\right)$, além de ser limitada em $\left(x_{\ell}, x\right)$. Assim, fica bem definida a função

$$
\hat{u}(x, j, k) \doteq \int_{x_{\ell}}^{x} \exp \left\{-k \int_{x^{\prime}}^{x} \frac{b+i a}{a^{2}+b^{2}}(s) d s+i \lambda j\left(x^{\prime}-x\right)\right\} \hat{f}\left(x^{\prime}, j, k\right) / c\left(x^{\prime}\right) d x^{\prime}
$$

para $x \in\left(x_{\ell}, x_{\ell+1}\right), j \in \mathbb{Z}$ e $k \in \mathbb{N}$.

O próximo passo será verificar que $\hat{u}(\cdot, j, k) \in \mathcal{C}^{\infty}\left(\left(x_{\ell}, x_{\ell+1}\right)\right)$. Para tanto, seja $\eta \in$ $\left(x_{\ell}, x_{\ell+1}\right)$ e considere a função

$$
\theta_{j k}(x) \doteq i \int_{\eta}^{x}(k / c(s)+\lambda j) d s=\int_{\eta}^{x}\left(k \frac{b+i a}{a^{2}+b^{2}}(s)+i \lambda j\right) d s, x \in\left(x_{\ell}, x_{\ell+1}\right)
$$

Podemos reescrever $\hat{u}(\cdot, j, k)$ da seguinte maneira

$$
\hat{u}(x, j, k)=\exp \left\{-\theta_{j k}(x)\right\} \int_{x_{\ell}}^{x} \exp \left\{\theta_{j k}\left(x^{\prime}\right)\right\} \hat{f}\left(x^{\prime}, j, k\right) / c\left(x^{\prime}\right) d x^{\prime}, x \in\left(x_{\ell}, x_{\ell+1}\right) .
$$

Como $b \geqslant 0$ em $\left(x_{\ell}, x_{\ell+1}\right), k>0$ e como

$$
\left|\exp \left\{\theta_{j k}\left(x^{\prime}\right)\right\}\right|=\exp \left\{k \int_{\eta}^{x^{\prime}} \frac{b}{a^{2}+b^{2}}(s) d s\right\}
$$

segue que a função

$$
\exp \left\{\theta_{j k}\left(x^{\prime}\right)\right\} \hat{f}\left(x^{\prime}, j, k\right) / c\left(x^{\prime}\right)
$$

é suave e limitada em $\left(x_{\ell}, x_{\ell+1}-\epsilon\right)$, para todo $\epsilon>0$ suficientemente pequeno. Assim, o Teorema Fundamental do Cálculo implica que

$$
\int_{x_{\ell}}^{x} \exp \left\{\theta_{j k}\left(x^{\prime}\right)\right\} \hat{f}\left(x^{\prime}, j, k\right) / c\left(x^{\prime}\right) d x^{\prime}
$$


é uma função suave em $\left(x_{\ell}, x_{\ell+1}-\epsilon\right)$, qualquer que seja $\epsilon>0$ suficientemente pequeno; $\operatorname{logo} \hat{u}(\cdot, j, k) \in \mathcal{C}^{\infty}\left(\left(x_{\ell}, x_{\ell+1}\right)\right)$ e

$$
\begin{aligned}
\partial_{x} \hat{u}(x, j, k) & =-\theta_{j k}^{\prime}(x) \hat{u}(x, j, k)+\hat{f}(x, j, k) / c(x) \\
& =-i(k / c(x)+\lambda j) \hat{u}(x, j, k)+\hat{f}(x, j, k) / c(x),
\end{aligned}
$$

ou seja, $\hat{u}(\cdot, j, k)$ é solução da equação (4.23).

Mostremos agora que $\hat{u}(\cdot, j, k)\left((j, k) \in \mathbb{Z}^{2}, k>0\right)$ é flat em $\left\{x_{\ell}, x_{\ell+1}\right\}$.

Para $x \in\left(x_{\ell}, x_{\ell+1}\right)$, temos

$$
\begin{aligned}
|\hat{u}(x, j, k)| & \leqslant \int_{x_{\ell}}^{x} \exp \left\{-k \int_{x^{\prime}}^{x} \frac{b}{a^{2}+b^{2}}(s) d s\right\}\left|\hat{f}\left(x^{\prime}, j, k\right) / c\left(x^{\prime}\right)\right| d x^{\prime} \\
& \leqslant \int_{x_{\ell}}^{x}\left|\hat{f}\left(x^{\prime}, j, k\right) / c\left(x^{\prime}\right)\right| d x^{\prime}
\end{aligned}
$$

e como $\hat{f}(\cdot, j, k)$ é flat em $c^{-1}(0)$, utilizando a fórmula de Taylor com resto integral e considerando $g \doteq f / c$, escrevemos

$$
\hat{f}\left(x^{\prime}, j, k\right) / c\left(x^{\prime}\right)=\hat{g}\left(x^{\prime}, j, k\right)=\frac{\left(x^{\prime}-x_{\ell}\right)^{n}}{(n-1) !} \int_{0}^{1}(1-t)^{n-1} \partial_{x}^{n} \hat{g}\left(x_{\ell}+t\left(x^{\prime}-x_{\ell}\right), j, k\right) d t .
$$

Assim,

$$
\left|\hat{f}\left(x^{\prime}, j, k\right) / c\left(x^{\prime}\right)\right| \leqslant \frac{\left|x^{\prime}-x_{\ell}\right|^{n}}{(n-1) !}\left\|\partial_{x}^{n} \hat{g}(\cdot, j, k)\right\|_{\infty} \leqslant \frac{\left|x^{\prime}-x_{\ell}\right|^{n}}{(n-1) !}\left\|\partial_{x}^{n} g\right\|_{\infty}
$$

onde obtemos a constante positiva $C_{n}=\frac{\left\|\partial_{x}^{n} g\right\|_{\infty}}{(n-1) !}$, que não depende de $(j, k)$ e satisfaz

$$
|\hat{u}(x, j, k)| \leqslant \frac{C_{n}\left|x-x_{\ell}\right|^{n+1}}{n+1}=O\left(\left|x-x_{\ell}\right|^{n+1}\right),
$$

para todo $n \in \mathbb{Z}_{+}$.

Em particular, $\hat{u}\left(x_{\ell}, j, k\right)=0$.

Olhemos agora perto de $x_{\ell+1}$. Para $h<0$ e $|h|$ suficientemente pequeno, temos

$$
\begin{gathered}
\hat{u}\left(x_{\ell+1}+h, j, k\right)= \\
\int_{x_{\ell}}^{x_{\ell+1}+h} \exp \left\{-k \int_{x^{\prime}}^{x_{\ell+1}+h} \frac{b+i a}{a^{2}+b^{2}}(s) d s+i \lambda j\left(x^{\prime}-x_{\ell+1}-h\right)\right\} \hat{f}\left(x^{\prime}, j, k\right) / c\left(x^{\prime}\right) d x^{\prime}=
\end{gathered}
$$




$$
\begin{aligned}
& \int_{x_{\ell}}^{x_{\ell+1}+2 h} \exp \left\{-k \int_{x^{\prime}}^{x_{\ell+1}+h} \frac{b+i a}{a^{2}+b^{2}}(s) d s+i \lambda j\left(x^{\prime}-x_{\ell+1}-h\right)\right\} \hat{f}\left(x^{\prime}, j, k\right) / c\left(x^{\prime}\right) d x^{\prime}+ \\
& \int_{x_{\ell+1}+2 h}^{x_{\ell+1}+h} \exp \left\{-k \int_{x^{\prime}}^{x_{\ell+1}+h} \frac{b+i a}{a^{2}+b^{2}}(s) d s+i \lambda j\left(x^{\prime}-x_{\ell+1}-h\right)\right\} \hat{f}\left(x^{\prime}, j, k\right) / c\left(x^{\prime}\right) d x^{\prime} .
\end{aligned}
$$

Vamos estimar esses dois últimos termos. O primeiro deles satisfaz

$$
\begin{aligned}
& \left|\int_{x_{\ell}}^{x_{\ell+1}+2 h} \exp \left\{-k \int_{x^{\prime}}^{x_{\ell+1}+h} \frac{b+i a}{a^{2}+b^{2}}(s) d s+i \lambda j\left(x^{\prime}-x_{\ell+1}-h\right)\right\} \hat{f}\left(x^{\prime}, j, k\right) / c\left(x^{\prime}\right) d x^{\prime}\right| \leqslant \\
& \|f / c\|_{\infty} \int_{x_{\ell}}^{x_{\ell+1}+2 h} \exp \left\{-k \int_{x^{\prime}}^{x_{\ell+1}+2 h} \frac{b}{a^{2}+b^{2}}(s) d s\right\} \exp \left\{-k \int_{x_{\ell+1}+2 h}^{x_{\ell+1}+h} \frac{b}{a^{2}+b^{2}}(s) d s\right\} d x^{\prime} .
\end{aligned}
$$

Para $h<0$ e $|h|$ suficientemente pequeno, as hipóteses $b \geqslant 0$ e $k>0$ implicam que

$$
\exp \left\{-k \int_{x^{\prime}}^{x_{\ell+1}+2 h} \frac{b}{a^{2}+b^{2}}(s) d s\right\} \leqslant 1, \quad x^{\prime} \in\left(x_{\ell}, x_{\ell+1}+2 h\right) \text {. }
$$

Assim,

$$
\begin{gathered}
\left|\int_{x_{\ell}}^{x_{\ell+1}+2 h} \exp \left\{-k \int_{x^{\prime}}^{x_{\ell+1}+h} \frac{b+i a}{a^{2}+b^{2}}(s) d s+i \lambda j\left(x^{\prime}-x_{\ell+1}-h\right)\right\} \hat{f}\left(x^{\prime}, j, k\right) / c\left(x^{\prime}\right) d x^{\prime}\right| \leqslant \\
\|f / c\|_{\infty} \int_{x_{\ell}}^{x_{\ell+1}+2 h} \exp \left\{-k \int_{x_{\ell+1}+2 h}^{x_{\ell+1}+h} \frac{b}{a^{2}+b^{2}}(s) d s\right\} d x^{\prime}
\end{gathered}
$$

e como $m_{\ell+1}<\infty$ e $2 \leqslant m_{\ell+1}<2 n_{\ell+1}-1$, podemos escrever

$$
\frac{b}{a^{2}+b^{2}}(s)=\left(x_{\ell+1}-s\right)^{-\rho} \beta(s), \quad(\rho \geqslant 2)
$$

para todo $s$ em um intervalo $\left(x_{\ell+1}-\delta, x_{\ell+1}\right)$, onde $\beta\left(x_{\ell+1}\right) \neq 0$ e $0<r \leqslant \beta(s) \leqslant M$. Desta forma, para $|h|$ suficientemente pequeno, obtemos

$$
\begin{gathered}
\|f / c\|_{\infty} \int_{x_{\ell}}^{x_{\ell+1}+2 h} \exp \left\{-k \int_{x_{\ell+1}+2 h}^{x_{\ell+1}+h} \frac{b}{a^{2}+b^{2}}(s) d s\right\} d x^{\prime} \leqslant \\
\|f / c\|_{\infty}\left|x_{\ell+1}-x_{\ell}\right| \exp \left\{\frac{-k r}{(\rho-1)}\left(\frac{1}{\left(x_{\ell+1}-s\right)^{\rho-1}}\right)_{x_{\ell+1}+2 h}^{x_{\ell+1}+h}\right\}= \\
\|f / c\|_{\infty}\left|x_{\ell+1}-x_{\ell}\right| \exp \left\{\frac{-k r}{(\rho-1)}\left(\frac{2^{\rho-1}-1}{2^{\rho-1}|h|^{\rho-1}}\right)\right\} \leqslant
\end{gathered}
$$




$$
\|f / c\|_{\infty}\left|x_{\ell+1}-x_{\ell}\right| \exp \left\{\frac{-r}{(\rho-1)}\left(\frac{2^{\rho-1}-1}{2^{\rho-1}|h|^{\rho-1}}\right)\right\}=O\left(|h|^{n}\right),
$$

para todo $n \in \mathbb{N}$, uma vez que $\rho>1$ e $r>0$ (veja Lema 1.8).

Para o segundo termo, temos a estimativa

$$
\begin{gathered}
\left|\int_{x_{\ell+1}+2 h}^{x_{\ell+1}+h} \exp \left\{-k \int_{x^{\prime}}^{x_{\ell+1}+h} \frac{b+i a}{a^{2}+b^{2}}(s) d s+i \lambda j\left(x^{\prime}-x_{\ell+1}-h\right)\right\} \hat{f}\left(x^{\prime}, j, k\right) / c\left(x^{\prime}\right) d x^{\prime}\right| \leqslant \\
\int_{x_{\ell+1}+2 h}^{x_{\ell+1}+h}\left|\hat{f}\left(x^{\prime}, j, k\right) / c\left(x^{\prime}\right)\right| d x^{\prime} \leqslant \\
C_{n} \int_{x_{\ell+1}+2 h}^{x_{\ell+1}+h}\left(x_{\ell+1}-x^{\prime}\right)^{n} d x^{\prime}=\frac{\left(2^{n+1}-1\right) C_{n}}{n+1}|h|^{n+1}=O\left(|h|^{n+1}\right),
\end{gathered}
$$

para todo $n \in \mathbb{Z}_{+}$, onde utilizamos novamente a fórmula de Taylor (agora em uma vizinhança de $x_{\ell+1}$ ) para obter a constante positiva

$$
C_{n}=\frac{\left\|\partial_{x}^{n} g\right\|_{\infty}}{(n-1) !}
$$

a qual não depende de $(j, k)$, como em (4.26).

Das estimativas (4.27) e (4.28) segue que

$$
\begin{aligned}
& \left|\hat{u}\left(x_{\ell+1}+h, j, k\right)\right| \leqslant \\
& \quad\|f / c\|_{\infty}\left|x_{\ell+1}-x_{\ell}\right| \exp \left\{\frac{-r}{(\rho-1)}\left(\frac{2^{\rho-1}-1}{2^{\rho-1}|h|^{\rho-1}}\right)\right\}+\frac{\left(2^{n+1}-1\right) C_{n}}{n+1}|h|^{n+1}=O\left(|h|^{n}\right),
\end{aligned}
$$

para todo $n \in \mathbb{Z}_{+}$.

Em particular, de (4.29) segue que $\hat{u}\left(x_{\ell+1}, j, k\right)=0$.

Em resumo, mostramos que $\hat{u}(\cdot, j, k)(j \in \mathbb{Z}$ e $k \in \mathbb{N})$ é uma função suave em $\left(x_{\ell}, x_{\ell+1}\right)$, solução de $(4.23)$, a qual se estende a uma função contínua em $\left[x_{\ell}, x_{\ell+1}\right]$ e tal que $\hat{u}\left(x_{\ell}, j, k\right)=0=\hat{u}\left(x_{\ell+1}, j, k\right)$. Mais ainda, utilizando a identidade (4.23) e um processo recursivo, verifica-se que estimativas análogas a (4.26) e (4.29) valem para as derivadas $\partial_{x}^{m} \hat{u}(\cdot, j, k), m \in \mathbb{N}$. Consequentemente, $\hat{u}(\cdot, j, k)$ (dada por (4.24)) se estende a uma função suave em $\left[x_{\ell}, x_{\ell+1}\right]$, a qual é flat em $\left\{x_{\ell}, x_{\ell+1}\right\}$. 
O próximo passo será resolver (4.23) para $k<0$, supondo ainda que $b \geqslant 0$ em $\left(x_{\ell}, x_{\ell+1}\right)$. Fixado $x \in\left(x_{\ell}, x_{\ell+1}\right)$, note que a função

$$
\left(x, x_{\ell+1}\right) \ni x^{\prime} \mapsto \exp \left\{-k \int_{x^{\prime}}^{x} \frac{b+i a}{a^{2}+b^{2}}(s) d s+i \lambda j\left(x^{\prime}-x\right)\right\} \hat{f}\left(x^{\prime}, j, k\right) / c\left(x^{\prime}\right)
$$

está bem definida, além de ser suave e limitada em $\left(x, x_{\ell+1}\right)$. Assim, podemos definir

$$
\hat{u}(x, j, k) \doteq-\int_{x}^{x_{\ell+1}} \exp \left\{-k \int_{x^{\prime}}^{x} \frac{b+i a}{a^{2}+b^{2}}(s) d s+i \lambda j\left(x^{\prime}-x\right)\right\} \hat{f}(x, j, k) / c\left(x^{\prime}\right) d x^{\prime}
$$

para $x \in\left(x_{\ell}, x_{\ell+1}\right)$ e $k<0$.

Como antes, vamos reescrever $\hat{u}(\cdot, j, k)$ para mostrar que tal função é suave em $\left(x_{\ell}, x_{\ell+1}\right)$. Utilizando novamente a função

$$
\theta_{j k}(x)=i \int_{\eta}^{x}(k / c(s)+\lambda j) d s=\int_{\eta}^{x}\left(k \frac{b+i a}{a^{2}+b^{2}}(s)+i \lambda j\right) d s, x \in\left(x_{\ell}, x_{\ell+1}\right),
$$

reescrevemos $\hat{u}(\cdot, j, k)$ na forma

$$
\hat{u}(x, j, k)=-\exp \left\{-\theta_{j k}(x)\right\} \int_{x}^{x_{\ell+1}} \exp \left\{\theta_{j k}\left(x^{\prime}\right)\right\} \hat{f}\left(x^{\prime}, j, k\right) / c\left(x^{\prime}\right) d x^{\prime}, x \in\left(x_{\ell}, x_{\ell+1}\right) .
$$

Como $b \geqslant 0$ em $\left(x_{\ell}, x_{\ell+1}\right)$ e $k<0$, para todo $\epsilon>0$ suficientemente pequeno, a função

$$
\exp \left\{\theta_{j k}\left(x^{\prime}\right)\right\} \hat{f}\left(x^{\prime}, j, k\right) / c\left(x^{\prime}\right)
$$

é suave e limitada em $\left(x_{\ell}+\epsilon, x_{\ell+1}\right)$. Assim, do Teorema Fundamental do Cálculo segue que

$$
\int_{x}^{x_{\ell+1}} \exp \left\{\theta_{j k}\left(x^{\prime}\right)\right\}\left(\hat{f}_{j k} / c\right)\left(x^{\prime}\right) d x^{\prime}
$$

é suave em $\left(x_{\ell}+\epsilon, x_{\ell+1}\right)$, qualquer que seja $\epsilon>0$ suficientemente pequeno; $\operatorname{logo} \hat{u}(\cdot, j, k) \epsilon$ $\mathcal{C}^{\infty}\left(\left(x_{\ell}, x_{\ell+1}\right)\right)$ e, além disso, temos

$$
\begin{aligned}
\partial_{x} \hat{u}(x, j, k) & =-\theta_{j k}^{\prime}(x) \hat{u}(x, j, k)+\hat{f}(x, j, k) / c(x) \\
& =-i(k / c(x)+\lambda j) \hat{u}(x, j, k)+\hat{f}(x, j, k) / c(x),
\end{aligned}
$$

ou seja, $\hat{u}(\cdot, j, k)$ é uma solução de (4.23).

Afirmamos que a solução $\hat{u}(\cdot, j, k)(k<0)$ é flat em $\left\{x_{\ell}, x_{\ell+1}\right\}$. 
Note que, como em (4.26), obtemos

$$
\begin{aligned}
|\hat{u}(x, j, k)| & \leqslant \int_{x}^{x_{\ell+1}} \exp \left\{-k \int_{x^{\prime}}^{x} \frac{b}{a^{2}+b^{2}}(s) d s\right\}\left|\hat{f}\left(x^{\prime}, j, k\right) / c\left(x^{\prime}\right)\right| d x^{\prime} \\
& \leqslant \int_{x}^{x_{\ell+1}}\left|\hat{f}\left(x^{\prime}, j, k\right) / c\left(x^{\prime}\right)\right| d x^{\prime} \leqslant \frac{C_{n}\left|x-x_{\ell+1}\right|^{n+1}}{n+1}=O\left(\left|x-x_{\ell+1}\right|^{n}\right),
\end{aligned}
$$

para todo $n \in \mathbb{Z}_{+}$.

Agora vamos estimar $|\hat{u}(x, j, k)|$, para $x$ próximo de $x_{\ell}$.

Para $h>0$ suficientemente pequeno, temos

$$
\begin{aligned}
&\left|\hat{u}\left(x_{\ell}+h, j, k\right)\right| \leqslant \int_{x_{\ell}+h}^{x_{\ell+1}} \exp \left\{-k \int_{x^{\prime}}^{x_{\ell}+h} \frac{b}{a^{2}+b^{2}}(s) d s\right\}\left|\hat{f}\left(x^{\prime}, j, k\right) / c\left(x^{\prime}\right)\right| d x^{\prime}= \\
& \int_{x_{\ell}+h}^{x_{\ell}+2 h} \exp \left\{-k \int_{x^{\prime}}^{x_{\ell}+h} \frac{b}{a^{2}+b^{2}}(s) d s\right\}\left|\hat{f}\left(x^{\prime}, j, k\right) / c\left(x^{\prime}\right)\right| d x^{\prime}+ \\
& \int_{x_{\ell}+2 h}^{x_{\ell+1}} \exp \left\{-k \int_{x^{\prime}}^{x_{\ell}+h} \frac{b}{a^{2}+b^{2}}(s) d s\right\}\left|\hat{f}\left(x^{\prime}, j, k\right) / c\left(x^{\prime}\right)\right| d x^{\prime}= \\
& \int_{x_{\ell}+h}^{x_{\ell}+2 h} \exp \left\{-k \int_{x^{\prime}}^{x_{\ell}+h} \frac{b}{a^{2}+b^{2}}(s) d s\right\}\left|\hat{f}\left(x^{\prime}, j, k\right) / c\left(x^{\prime}\right)\right| d x^{\prime}+ \\
& \int_{x_{\ell}+2 h}^{x_{\ell+1}} \exp \left\{-k \int_{x^{\prime}}^{x_{\ell}+2 h} \frac{b}{a^{2}+b^{2}}(s) d s-k \int_{x_{\ell}+2 h}^{x_{\ell}+h} \frac{b}{a^{2}+b^{2}}(s) d s\right\}\left|\hat{f}\left(x^{\prime}, j, k\right) / c\left(x^{\prime}\right)\right| d x^{\prime} .
\end{aligned}
$$

Como antes (ver (4.28)), a primeira das duas integrais acima é estimada por

$$
\frac{\left(2^{n+1}-1\right) C_{n}}{n+1}|h|^{n+1}=O\left(|h|^{n+1}\right),
$$

para todo $n \in \mathbb{N}$, ao passo que, para $h>0$ suficientemente pequeno, a segunda integral possui a estimativa

$$
\begin{gathered}
\int_{x_{\ell}+2 h}^{x_{\ell+1}} \exp \left\{-k \int_{x^{\prime}}^{x_{\ell}+2 h} \frac{b}{a^{2}+b^{2}}(s) d s-k \int_{x_{\ell}+2 h}^{x_{\ell}+h} \frac{b}{a^{2}+b^{2}}(s) d s\right\}\left|\hat{f}\left(x^{\prime}, j, k\right) / c\left(x^{\prime}\right)\right| d x^{\prime} \leqslant \\
\int_{x_{\ell}+2 h}^{x_{\ell+1}} \exp \left\{-k \int_{x_{\ell}+2 h}^{x_{\ell}+h} \frac{b}{a^{2}+b^{2}}(s) d s\right\}\left|\hat{f}\left(x^{\prime}, j, k\right) / c\left(x^{\prime}\right)\right| d x^{\prime},
\end{gathered}
$$

uma vez que $k<0$ e $b \geqslant 0$ nos fornece o controle

$$
\exp \left\{-k \int_{x^{\prime}}^{x_{\ell}+2 h} \frac{b}{a^{2}+b^{2}}(s) d s\right\} \leqslant 1, \quad x^{\prime} \in\left(x_{\ell}+2 h, x_{\ell+1}\right) .
$$


Assim, como em (4.27), a segunda integral é estimada por

$$
\begin{gathered}
\|f / c\|_{\infty}\left|x_{\ell+1}-x_{\ell}\right| \exp \left\{\frac{k r^{\prime}}{(\rho-1)}\left(\frac{2^{\rho-1}-1}{2^{\rho-1} h^{\rho-1}}\right)\right\} \leqslant \\
\|f / c\|_{\infty}\left|x_{\ell+1}-x_{\ell}\right| \exp \left\{\frac{-r^{\prime}}{(\rho-1)}\left(\frac{2^{\rho-1}-1}{2^{\rho-1} h^{\rho-1}}\right)\right\}=O\left(|h|^{n}\right), n \in \mathbb{N},
\end{gathered}
$$

onde escrevemos $\frac{b}{a^{2}+b^{2}}(s)=\left(s-x_{\ell}\right)^{-\rho} \alpha(s)$ em um intervalo $\left(x_{\ell}, x_{\ell}+\delta\right)$, com $\rho \geqslant 2$, $\alpha\left(x_{\ell}\right) \neq 0$ e $0<r^{\prime} \leqslant \alpha(s) \leqslant M^{\prime}$; uma vez que $m_{\ell}<\infty$ e $2 \leqslant m_{\ell}<2 n_{\ell}-1$.

Das estimativas (4.32) e (4.33) obtemos

$$
\begin{aligned}
& \left|\hat{u}\left(x_{\ell}+h, j, k\right)\right| \leqslant \\
& \frac{\left(2^{n+1}-1\right) C_{n}}{n+1}|h|^{n+1}+\|f / c\|_{\infty}\left|x_{\ell+1}-x_{\ell}\right| \exp \left\{\frac{-r^{\prime}}{(\rho-1)}\left(\frac{2^{\rho-1}-1}{2^{\rho-1} h^{\rho-1}}\right)\right\}=O\left(|h|^{n}\right),
\end{aligned}
$$

para todo $n \in \mathbb{Z}_{+}$.

Portanto, das estimativas (4.31) e (4.34) segue que a função $\hat{u}(\cdot, j, k)(k<0)$ se estende a uma função contínua em $\left[x_{\ell}, x_{\ell+1}\right]$, a qual satisfaz $\hat{u}\left(x_{\ell}, j, k\right)=0=\hat{u}\left(x_{\ell+1}, j, k\right)$. Mais ainda, utilizando a identidade (4.23) e um processo recursivo obtemos estimativas análogas para todas as derivadas $\partial_{x}^{m} \hat{u}(\cdot, j, k), m \in \mathbb{N}$; logo $\hat{u}(\cdot, j, k)$ (dada por (4.30)) pode ser estendida a uma função suave em $\left[x_{\ell}, x_{\ell+1}\right]$, a qual é flat em $\left\{x_{\ell}, x_{\ell+1}\right\}$.

Agora vamos dizer como construir soluções para (4.23) no caso em que $b \leqslant 0$ em $\left(x_{\ell}, x_{\ell+1}\right)$. Neste caso, para $k<0$ definimos $\hat{u}(\cdot, j, k)$ como em (4.24), enquanto que para $k>0$ definimos $\hat{u}(\cdot, j, k)$ como em (4.30). Como antes, obtemos uma sequência de soluções $\hat{u}(\cdot, j, k)$ que são infinitamente diferenciáveis em $\left[x_{\ell}, x_{\ell+1}\right]$ e flat em $\left\{x_{\ell}, x_{\ell+1}\right\}$.

Como o intervalo $\left(x_{\ell}, x_{\ell+1}\right)$, com $\ell \in\{1, \ldots, N\}$, foi tomado de maneira arbitrária e como as soluções construídas nesses intervalos são flat em $\left\{x_{\ell}, x_{\ell+1}\right\}$, podemos definir, para cada $(j, k) \in \mathbb{Z}^{2}$ com $k \neq 0$, uma solução $\hat{u}(\cdot, j, k)$ de $(4.20)$ em $\mathcal{C}^{\infty}\left(\mathbb{T}^{1}\right)$.

Para concluir a demonstração precisamos provar que $(\hat{u}(\cdot, j, k))_{(j, k) \in \mathbb{Z} \times(\mathbb{Z} \backslash\{0\})}$ é rapidamente decrescente. Precisamos mostrar que, para todo $\gamma \in \mathbb{Z}_{+}$e $n \in \mathbb{Z}_{+}$, existe $C=C(\gamma, n)>0$ tal que

$$
(|j|+|k|)^{\gamma}\left|\hat{u}^{(n)}(x, j, k)\right| \leqslant C
$$


para todo $x \in \mathbb{T}^{1}$ e todo $(j, k) \in \mathbb{Z} \times(\mathbb{Z} \backslash\{0\})$. Note que é suficiente encontrar, para cada $\ell \in\{1, \ldots, N\}$, uma constante $C=C(\ell, \gamma, n)>0$ tal que

$$
(|j|+|k|)^{\gamma}\left|\hat{u}^{(n)}(x, j, k)\right| \leqslant C,
$$

para todo $x \in\left[x_{\ell}, x_{\ell+1}\right]$ e todo $(j, k) \in \mathbb{Z} \times(\mathbb{Z} \backslash\{0\})$.

Suponha que $b \geqslant 0$ em $\left[x_{\ell}, x_{\ell+1}\right]$ (o caso em que $b \leqslant 0$ é análogo). Da definição das funções $\hat{u}(\cdot, j, k)$ (ver (4.24) e (4.30)) segue que é suficiente provar o seguinte: quaisquer que sejam $\gamma_{1} \in \mathbb{Z}_{+}, \gamma_{2} \in \mathbb{Z}_{+}$e $\gamma_{3} \in \mathbb{Z}_{+}$, existe $C=C\left(\gamma_{1}, \gamma_{2}, \gamma_{3}, \ell\right)>0$ tal que

$$
|j|^{\gamma_{1}}|k|^{\gamma_{2}}|c(x)|^{-\gamma_{3}}|\hat{u}(x, j, k)| \leqslant C
$$

para todo $x \in\left[x_{\ell}, x_{\ell+1}\right]$ e $(j, k) \in \mathbb{Z} \times(\mathbb{Z} \backslash\{0\})$.

Denotando $g=f / c \in \mathcal{C}^{\infty}\left(\mathbb{T}^{3}\right)$, temos $\hat{g}(x, j, k)=\hat{f}(x, j, k) / c(x)$ e $j^{\gamma_{1}} k^{\gamma_{2}} \hat{g}(x, j, k)=$ $(-1)^{\gamma_{1}+\gamma_{2}}\left(\partial^{\left(0, \gamma_{1}, \gamma_{2}\right)} g\right) \hat{(}(x, j, k)$. Assim, para $k>0$ a definição (4.24) implica que

$$
\begin{gathered}
|j|^{\gamma_{1}}|k|^{\gamma_{2}}|c(x)|^{-\gamma_{3}}|\hat{u}(x, j, k)|= \\
\left.|c(x)|^{-\gamma_{3}} \mid \int_{x_{\ell}}^{x} \exp \left\{-k \int_{x^{\prime}}^{x} \frac{b+i a}{a^{2}+b^{2}}(s) d s+i \lambda j\left(x^{\prime}-x\right)\right\}\left(\partial^{\left(0, \gamma_{1}, \gamma_{2}\right)} g\right) \hat{(} x^{\prime}, j, k\right) d x^{\prime} \mid .
\end{gathered}
$$

Mais ainda, procedendo como em (4.26), (4.27) e (4.28), com $\left(\partial^{\left(0, \gamma_{1}, \gamma_{2}\right)} g\right) \hat{)}\left(x^{\prime}, j, k\right)$ no lugar de $\hat{f}\left(x^{\prime}, j, k\right) / c\left(x^{\prime}\right)$, obtemos uma constante $C_{1}>0$ (que não depende de $(j, k)$ ) tal que

$$
|c(x)|^{-\gamma_{3}}\left|\int_{x_{\ell}}^{x} \exp \left\{-k \int_{x^{\prime}}^{x} \frac{b+i a}{a^{2}+b^{2}}(s) d s+i \lambda j\left(x^{\prime}-x\right)\right\}\left(\partial^{\left(0, \gamma_{1}, \gamma_{2}\right)} g\right) \hat{(}\left(x^{\prime}, j, k\right) d x^{\prime}\right| \leqslant C_{1},
$$

quaisquer que sejam $x \in\left(x_{\ell}, x_{\ell+1}\right)$ e $(j, k) \in \mathbb{Z} \times \mathbb{N}$. Note que, olhando em um intervalo $\left[x_{\ell}+\delta, x_{\ell+1}-\delta\right]$, a existência de tal constante $C_{1}$ segue do fato de que $|c(x)|^{-\gamma_{3}}$ é limitada

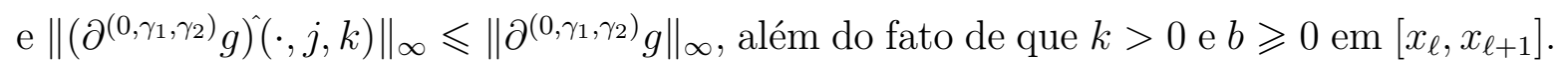

Analogamente, para $k<0$, usamos a definição (4.30) e procedemos como em (4.31), (4.32) e (4.33), obtendo uma constante $C_{2}>0$ (que não depende de $(j, k)$ ) tal que

$$
|j|^{\gamma_{1}}|k|^{\gamma_{2}}|c(x)|^{-\gamma_{3}}|\hat{u}(x, j, k)| \leqslant C_{2}
$$

para todo $x \in\left(x_{\ell}, x_{\ell+1}\right)$ e para todo $(j, k) \in \mathbb{Z} \times\left(\mathbb{Z}_{-} \backslash\{0\}\right)$. 
Portanto, a sequência $\left(\hat{u}_{j k}\right)_{(j, k) \in \mathbb{Z} \times(\mathbb{Z} \backslash\{0\})}$ decai rapidamente.

O que conclui a demonstração da Proposição 4.17.

O próximo resultado generaliza a proposição anterior, permitindo que $b$ mude de sinal (no máximo uma vez) em cada intervalo $\left(x_{\ell}, x_{\ell+1}\right)$.

Proposição 4.18. Suponha que c possui apenas anulamentos de ordem finita e que $\emptyset \neq c^{-1}(0)=\mathrm{V}_{1}$, como em (4.19). Suponha ainda que $\lambda$ não é um número de Liouville e que, para cada $\ell \in\{1, \ldots, N\}$, existe $\eta \in\left(x_{\ell}, x_{\ell+1}\right)$ tal que $b$ não muda de sinal em cada um dos intervalos $\left(x_{\ell}, \eta\right)$ e $\left(\eta, x_{\ell+1}\right)$. Nestas condições, para cada $f \in\left(\operatorname{ker}^{t} \mathbf{L}\right)^{\circ}$ que é flat em $c^{-1}(0) \times \mathbb{T}^{2}$, existe $u \in \mathcal{C}^{\infty}\left(\mathbb{T}^{3}\right)$ tal que $\mathrm{L} u=f$.

Demonstração: Seja $f \in\left(\operatorname{ker}^{t} \mathbf{L}\right)^{\circ}, f$ flat em $c^{-1}(0)$. Vamos procurar uma solução de $\mathrm{L} u=f$ em $\mathcal{C}^{\infty}\left(\mathbb{T}^{3}\right)$. Utilizando série parcial de Fourier nas variáveis $(y, t)$, segue que basta encontrar uma sequência rapidamente decrescente $\hat{u}(\cdot, j, k)_{(j, k) \in \mathbb{Z}^{2}} \subset \mathcal{C}^{\infty}\left(\mathbb{T}^{1}\right)$, tal que cada $\hat{u}(\cdot, j, k)$ seja solução de

$$
c(x) \partial_{x} \hat{u}(x, j, k)+i(k+c(x) \lambda j) \hat{u}(x, j, k)=\hat{f}(x, j, k), \quad x \in \mathbb{T}^{1} .
$$

Quando $k=0$, procedendo como na demonstração da Proposição (4.17), obtemos uma sequência $\hat{u}(\cdot, j, 0)$ que satisfaz as propriedades requeridas acima.

Para $k \neq 0$ e $j \in \mathbb{Z}$, mostraremos que (4.35) tem uma solução suave em cada intervalo $\left[x_{\ell}, x_{\ell+1}\right]$, a qual é flat em $\left\{x_{\ell}, x_{\ell+1}\right\}$, para $\ell=1, \ldots, N$.

Os casos em que $b \geqslant 0$ ou $b \leqslant 0$ em $\left(x_{\ell}, x_{\ell+1}\right)$, já foram feitos na demonstração da Proposição 4.17.

Suponha, agora, que existe $\eta \in\left(x_{\ell}, x_{\ell+1}\right)$ tal que $b \geqslant 0$ em $\left(x_{\ell}, \eta\right)$ e $b \leqslant 0$ em $\left(\eta, x_{\ell+1}\right)$.

A equação (4.35) restrita a $\left(x_{\ell}, x_{\ell+1}\right)$ torna-se

$$
\partial_{x} \hat{u}(x, j, k)+i(k / c(x)+\lambda j) \hat{u}(x, j, k)=\hat{f}(x, j, k) / c(x),
$$

para todo $(j, k) \in \mathbb{Z} \times(\mathbb{Z} \backslash\{0\})$.

Considere

$$
\theta_{j k}(x)=i \int_{\eta}^{x}\left(\frac{k}{c(s)}+\lambda j\right) d s=\int_{\eta}^{x}\left(k \frac{b+i a}{a^{2}+b^{2}}(s)+i \lambda j\right) d s .
$$


Para $k>0$, defina

$$
\hat{u}(x, j, k)=\exp \left\{-\theta_{j k}(x)\right\} \int_{x_{\ell}}^{x} \exp \left\{\theta_{j k}\left(x^{\prime}\right)\right\} \hat{f}\left(x^{\prime}, j, k\right) / c\left(x^{\prime}\right) d x^{\prime},
$$

para $x \in\left(x_{\ell}, x_{\ell+1}\right)$. Como $b \geqslant 0$ em $\left[x_{\ell}, \eta\right]$ e como $\hat{f}(\cdot, j, k)$ é flat em $x_{\ell}$, procedendo como na demonstração da Proposição 4.17 verifica-se que $\hat{u}(\cdot, j, k)$ pertence a $\mathcal{C}^{\infty}\left(\left[x_{\ell}, x_{\ell+1}\right)\right)$ e que $\hat{u}(\cdot, j, k)$ é flat em $x_{\ell}$. Além disso, $\hat{u}(\cdot, j, k)$ é solução de (4.36).

O próximo passo será mostrar que $\hat{u}(\cdot, j, k)$ também é flat em $x_{\ell+1}$. Para tanto, precisaremos reescrever a solução $\hat{u}(\cdot, j, k)$.

Como $b \geqslant 0$ em $\left(x_{\ell}, \eta\right)$ e $b \leqslant 0$ em $\left(\eta, x_{\ell+1}\right)$, a identidade

$$
\left|\exp \left\{\theta_{j k}(x)\right\}\right|=\left|\exp \left\{\int_{\eta}^{x} i\left(\frac{k}{c(s)}+\lambda j\right) d s\right\}\right|=\exp \left\{k \int_{\eta}^{x} \frac{b}{a^{2}+b^{2}}(s) d s\right\}
$$

implica que, para todo $k>0$, a função $\exp \left\{\theta_{j k}(x)\right\}$ é limitada em $\left(x_{\ell}, x_{\ell+1}\right)$. Mais ainda, como $m_{\ell}<\infty$ e $2 \leqslant m_{\ell}<2 n_{\ell}-1$, podemos escrever

$$
\frac{b}{a^{2}+b^{2}}(s)=\left(s-x_{\ell}\right)^{-\rho} \alpha(s), \quad(\rho \geqslant 2),
$$

para todo $s$ em um intervalo $\left(x_{\ell}, x_{\ell}+\delta\right)$, onde $\alpha\left(x_{\ell}\right) \neq 0$ e $0<r^{\prime} \leqslant \alpha(s) \leqslant M^{\prime}$. Sendo assim, de (4.38) segue que a função

$$
\psi_{j k}(x) \doteq\left\{\begin{array}{lll}
\exp \left\{\theta_{j k}(x)\right\}, & \text { se } & x \in\left(x_{\ell}, x_{\ell+1}\right) \\
0, & \text { se } \quad x \in \mathbb{T}^{1} \backslash\left(x_{\ell}, x_{\ell+1}\right)
\end{array}\right.
$$

é flat em $x_{\ell}$. Do mesmo modo, como $m_{\ell+1}<\infty$ e $2 \leqslant m_{\ell+1}<2 n_{\ell+1}-1$, verifica-se que $\psi_{j k}$ é flat em $x_{\ell+1}$. Assim, $\psi_{j k} \in \mathcal{C}^{\infty}\left(\mathbb{T}^{1}\right)$ e como $c$ só possui zeros de ordem finita, $\psi_{j k} / c \in \mathcal{C}^{\infty}\left(\mathbb{T}^{1}\right)$. Cálculos diretos mostram que $\left(\psi_{j k} / c\right)(x) e^{-i(j y+k t)}$ pertence a $\operatorname{ker}^{t} \mathrm{~L}$; $\log O$

$$
\int_{x_{\ell}}^{x_{\ell+1}} \exp \left\{\theta_{j k}(x)\right\} \hat{f}(x, j, k) / c(x) d x=0, \text { para todo } k>0 .
$$

Em particular, podemos reescrever cada solução $\hat{u}(\cdot, j, k)$ da seguinte maneira

$$
\hat{u}(x, j, k)=-\exp \left\{-\theta_{j k}(x)\right\} \int_{x}^{x_{\ell+1}} \exp \left\{\theta_{j k}\left(x^{\prime}\right)\right\} \hat{f}\left(x^{\prime}, j, k\right) / c\left(x^{\prime}\right) d x^{\prime} \text {, para } x \in\left(x_{\ell}, x_{\ell+1}\right) .
$$


Utilizando a expressão acima e os mesmos argumentos da demosntração da Proposição 4.17 , podemos verificar que $\hat{u}(\cdot, j, k)$ é flat em $x_{\ell+1}$. Portanto, $\hat{u}(\cdot, j, k)(k>0)$ é suave em $\left[x_{\ell}, x_{\ell+1}\right]$ e flat em $\left\{x_{\ell}, x_{\ell+1}\right\}$.

Para $k<0$, consideraremos a solução de (4.36) dada por

$$
\hat{u}(x, j, k)=\exp \left\{-\theta_{j k}(x)\right\} \int_{\eta}^{x} \exp \left\{\theta_{j k}\left(x^{\prime}\right)\right\} \hat{f}\left(x^{\prime}, j, k\right) / c\left(x^{\prime}\right) d x^{\prime}, \quad x \in\left(x_{\ell}, x_{\ell+1}\right) .
$$

É claro que $\hat{u}(\cdot, j, k)$ é suave em $\left(x_{\ell}, x_{\ell+1}\right)$ e seguindo novamente os argumentos da demonstração da Proposição 4.17 , verifica-se que $\hat{u}(\cdot, j, k)(k<0)$ é suave em $\left[x_{\ell}, x_{\ell+1}\right]$, flat em $\left\{x_{\ell}, x_{\ell+1}\right\}$ e satisfaz (4.36).

Suponha, agora, que existe $\eta \in\left(x_{\ell}, x_{\ell+1}\right)$ tal que $b \leqslant 0$ em $\left(x_{\ell}, \eta\right)$ e $b \geqslant 0$ em $\left(\eta, x_{\ell+1}\right)$. Neste caso, para $k>0$ definimos $\hat{u}(\cdot, j, k)$ como em (4.40), ou seja,

$$
\hat{u}(x, j, k)=\exp \left\{-\theta_{j k}(x)\right\} \int_{\eta}^{x} \exp \left\{\theta_{j k}\left(x^{\prime}\right)\right\} \hat{f}(x, j, k) / c\left(x^{\prime}\right) d x^{\prime}, \text { para } x \in\left(x_{\ell}, x_{\ell+1}\right)
$$

enquanto que para $k<0$ definimos $\hat{u}(\cdot, j, k)$ por

$$
\hat{u}(x, j, k)=-\exp \left\{-\theta_{j k}(x)\right\} \int_{x}^{x_{\ell+1}} \exp \left\{\theta_{j k}\left(x^{\prime}\right)\right\} \hat{f}(x, j, k) / c\left(x^{\prime}\right) d x^{\prime}, \text { para } x \in\left(x_{\ell}, x_{\ell+1}\right) .
$$

Procedendo como antes (demonstração da Proposição 4.17) segue que, para $k>0$, $\hat{u}(\cdot, j, k)$ define uma solução de (4.36), a qual é suave em $\left(x_{\ell}, x_{\ell+1}\right)$ e flat em $\left\{x_{\ell}, x_{\ell+1}\right\}$. Do mesmo modo, quando $k<0$, a solução $\hat{u}(\cdot, j, k)$ é suave em $\left(x_{\ell}, x_{\ell+1}\right)$ e flat em $x_{\ell+1}$. Mais ainda, procedendo como acima, temos

$$
\int_{x_{\ell}}^{x_{\ell+1}} \exp \left\{\theta_{j k}\left(x^{\prime}\right)\right\} \hat{f}\left(x^{\prime}, j, k\right) / c\left(x^{\prime}\right) d x^{\prime}=0, \quad \text { para todo } \quad k<0
$$

o que nos permite reescrever

$$
\hat{u}(x, j, k)=\exp \left\{-\theta_{j k}(x)\right\} \int_{x_{\ell}}^{x} \exp \left\{\theta_{j k}\left(x^{\prime}\right)\right\} \hat{f}\left(x^{\prime}, j, k\right) / c\left(x^{\prime}\right) d x^{\prime}, \text { para todo } x \in\left(x_{\ell}, x_{\ell+1}\right) .
$$

Utilizando essa última expressão e procedendo como na demonstração da Proposição 4.17 , verifica-se que $\hat{u}(\cdot, j, k)$ também é flat em $x_{\ell}$.

Como $\ell \in\{1, \ldots, N\}$ foi fixado arbitrariamente e como cada solução de (4.36) construída acima é flat em $\left\{x_{\ell}, x_{\ell+1}\right\}$, podemos colar essas soluções de modo a obtermos 
uma sequência $\hat{u}(\cdot, j, k)_{(j, k) \in \mathbb{Z} \times \mathbb{Z} \backslash\{0\}} \subset \mathcal{C}^{\infty}\left(\mathbb{T}^{1}\right)$ tal que cada termo $\hat{u}(\cdot, j, k)$ é solução de (4.35).

Por fim, aplicando os mesmos argumentos que foram utilizados na demonstração da Proposição 4.17, verifica-se que tal sequência é rapidamente decrescente.

Portanto, a demonstração da Proposição 4.18 está completa.

A próxima proposição mostra que, nas condições do Teorema 4.8, o fato de que $b$ muda de sinal no máximo uma vez em cada intervalo $\left(x_{\ell}, x_{\ell}+1\right)$ é uma condição necessária para a resolubilidade global de L.

Proposição 4.19. Suponha que $c^{-1}(0) \neq \emptyset$ e que c possui apenas zeros de ordem finita. Além disso, suponha que existe $\ell \in\{1, \ldots, N\}$ de modo que $m_{\ell}<\infty$ e $2 \leqslant m_{\ell} \leqslant 2 n_{\ell}-1$. Se $b$ mudar de sinal pelo menos duas vezes em $\left(x_{\ell}, x_{\ell+1}\right)$, onde $x_{\ell}$ e $x_{\ell+1}$ são zeros consecutivos de c, então L não é globalmente resolúvel.

Demonstração: Iremos construir uma função $f \in\left(\operatorname{ker}^{t} \mathrm{~L}\right)^{\circ} \backslash \mathrm{LC} \mathcal{C}^{\infty}\left(\mathbb{T}^{3}\right)$, a qual, por meio de sua série parcial de Fourier nas variáveis $(y, t)$, será escrita na forma

$$
f(x, y, t)=\sum_{k=1}^{\infty} \hat{f}(x, 0, k) e^{i k t}
$$

onde $\hat{f}(\cdot, 0, k) \in \mathcal{C}^{\infty}\left(\mathbb{T}^{1}\right)$ será escolhida a seguir. Note que a função $f$ depende apenas das variáveis $(x, t)$.

Como $m_{\ell}<\infty$, temos $b>0$ ou $b<0$ em algum intervalo $\left(x_{\ell}, x_{\ell}+\delta\right)$. Multiplicando $\mathrm{L}$ por -1 e efetuando a mudança de variáveis $(X, Y, T)=(x, y,-t)$, podemos supor que $b>0 \mathrm{em}\left(x_{\ell}, x_{\ell}+\delta\right)$.

Por hipótese, $b$ muda de sinal ao menos duas vezes em $\left(x_{\ell}, x_{\ell+1}\right)$; assim, podemos encontar pontos $x_{1}$ e $x_{2}$ tais que $x_{\ell}+\delta<x_{1}<x_{2}<x_{\ell+1}, b\left(x_{1}\right)<0$ e $b\left(x_{2}\right)>0$.

Definindo

$$
\sigma=\inf \left\{x \in\left(x_{1}, x_{2}\right) ; b(s) \geqslant 0, s \in\left(x, x_{2}\right)\right\}
$$

obtemos $b \geqslant 0$ em $\left(\sigma, x_{2}\right)$ e obtemos uma sequência $\sigma_{n} \rightarrow \sigma$, tal que $\sigma_{n}<\sigma$ e $b\left(\sigma_{n}\right)<0$, para todo $n \in \mathbb{N}$.

Fixemos $\psi_{1} \in \mathcal{C}_{c}^{\infty}\left(\left(\sigma, x_{2}\right)\right)$, uma função com as seguintes propriedades: $b>0 \mathrm{em}$ $\operatorname{supp} \psi_{1}, \psi_{1} \geqslant 0$ e $\int_{x_{\ell}}^{x_{\ell+1}} \psi_{1}=1$. 
Tomando $n$ suficientemente grande, obtemos um ponto $\eta \doteq \sigma_{n}<\sigma$ tal que $b(\eta)<0$ e $\int_{\eta}^{x} \frac{b}{a^{2}+b^{2}}(s) d s>0$, para todo $x \in \operatorname{supp} \psi_{1}$. Como $b(\eta)<0$, existe $\epsilon>0$ tal que $b<0$ em $(\eta, \eta+\epsilon)$. Agora fixe $\psi_{2} \in \mathcal{C}_{c}^{\infty}((\eta, \eta+\epsilon))$ tal que $\psi_{2} \geqslant 0$ e $\int_{x_{\ell}}^{x_{\ell+1}} \psi_{2}=1$.

Estamos quase prontos para definir as funções $\hat{f}(\cdot, 0, k)$. Para tanto, considere ainda $y_{0} \doteq \sup \operatorname{supp} \psi_{2}$ e $M \doteq-\int_{y_{0}}^{\eta+\epsilon} \frac{b}{a^{2}+b^{2}}(s) d s>0$.

Para $k>0$, defina

$$
\begin{aligned}
& \hat{f}(x, 0, k)= \\
& \quad c(x) e^{-k M}\left[\psi_{2}(x) \exp \left\{-i k \int_{\eta}^{x} \frac{a}{a^{2}+b^{2}}(s) d s\right\}+\psi_{1}(x) \exp \left\{-i k \int_{\eta}^{x} \frac{1}{c(s)} d s\right\} d_{k}\right],
\end{aligned}
$$

onde

$$
d_{k} \doteq-\int_{x_{\ell}}^{x_{\ell+1}} \psi_{2}(x) \exp \left\{k \int_{\eta}^{x} \frac{b}{a^{2}+b^{2}}(s) d s\right\} d x .
$$

Note que $\hat{f}(\cdot, 0, k) \equiv 0$ em uma vizinhança de $x_{\ell}$ e em uma vizinhança de $x_{\ell+1}$, pois

$$
\operatorname{supp} \hat{f}(\cdot, 0, k) \subset \operatorname{supp} \psi_{1} \cup \operatorname{supp} \psi_{2} \subset\left(x_{\ell}, x_{\ell+1}\right) \text {. }
$$

Afirmamos que a sequência $\hat{f}(\cdot, 0, k)$ decai rapidamente. De fato, note que

$$
\left|d_{k}\right| \leqslant \exp \left\{k \int_{\eta}^{\inf \operatorname{supp} \psi_{2}} \frac{b}{a^{2}+b^{2}}(s) d s\right\}<1 \text {, para todo } k>0
$$

e

$$
\left|\exp \left\{-i k \int_{\eta}^{x} \frac{1}{c(s)} d s\right\}\right|=\exp \left\{-k \int_{\eta}^{x} \frac{b}{a^{2}+b^{2}}(s) d s\right\}<1,
$$

para todo $x \in \operatorname{supp} \psi_{1}$ e $k>0$. Assim, o fator $e^{-k M}$ garante o decaimento rápido de $\hat{f}(\cdot, 0, k)$; consequentemente, (4.41) define uma função $f \mathrm{em} \mathcal{C}^{\infty}\left(\mathbb{T}^{1}\right)$.

O próximo passo será mostrar que $f$ pertence a $\left(\operatorname{ker}^{t} \mathrm{~L}\right)^{\circ}$. Seja $\mu$ uma distribuição em $\operatorname{ker}^{t} \mathrm{~L}$. Utilizando série parcial de Fourier nas variáveis $(y, t)$, obtemos

$$
\partial_{x}(c \hat{\mu}(\cdot, j, k))+i(k+\lambda j c) \hat{\mu}(\cdot, j, k) \equiv 0, \quad(j, k) \in \mathbb{Z}^{2}
$$

Como $\hat{f}(\cdot, j, k) \equiv 0$ se $j \neq 0$, temos $\langle\hat{\mu}(\cdot,-j,-k), \hat{f}(\cdot, j, k)\rangle \equiv 0$, para todo $j \neq 0$.

Quando $j=0$, obtemos

$$
\partial_{x}(c \hat{\mu}(\cdot, 0, k))+i k \hat{\mu}(\cdot, 0, k) \equiv 0, \quad k \in \mathbb{Z} .
$$


De (4.42) segue que, no intervalo $\left(x_{\ell}, x_{\ell+1}\right)$, temos

$$
\hat{\mu}(x, 0, k)=\frac{C_{k}}{c(x)} \exp \left\{-i k \int_{\eta}^{x} \frac{1}{c(s)} d s\right\} \quad\left(C_{k} \in \mathbb{C}\right)
$$

$\log O$

$$
\langle\mu(\cdot, 0, k), \phi\rangle=C_{k} \int_{0}^{2 \pi}(\phi / c)(x) \exp \left\{-i k \int_{\eta}^{x} \frac{1}{c(s)} d s\right\} d x
$$

para toda $\phi \in \mathcal{C}_{c}^{\infty}\left(\left(x_{\ell}, x_{\ell+1}\right)\right)$ e $k \in \mathbb{Z}$.

Como $\hat{f}(\cdot, 0, k) \in \mathcal{C}_{c}^{\infty}\left(\left(x_{\ell}, x_{\ell+1}\right)\right)$, temos

$$
\begin{gathered}
\langle\mu(\cdot, 0,-k), \hat{f}(\cdot, 0, k)\rangle=C_{-k} \int_{0}^{2 \pi} \exp \left\{i k \int_{\eta}^{x} \frac{1}{c(s)} d s\right\} \hat{f}(x, 0, k) / c(x) d x= \\
C_{-k} e^{-k M} \int_{0}^{2 \pi}\left[\psi_{2}(x) \exp \left\{k \int_{\eta}^{x} \frac{b}{a^{2}+b^{2}}(s) d s\right\}+d_{k} \psi_{1}(x)\right] d x=0 .
\end{gathered}
$$

Assim,

$$
\langle\mu, f\rangle=\sum_{k=1}^{\infty}(2 \pi)^{2}\langle\mu(\cdot, 0,-k), \hat{f}(\cdot, 0, k)\rangle=0
$$

qualquer que seja $\mu \in \operatorname{ker}^{t} \mathrm{~L}$; $\log 0 f \in\left(\operatorname{ker}^{t} \mathrm{~L}\right)^{\circ}$.

Para concluir a demonstração veremos que $L u=f$ não tem solução $u$ em $\mathcal{C}^{\infty}\left(\mathbb{T}^{3}\right)$. De fato, se existe $u \in \mathcal{C}^{\infty}\left(\mathbb{T}^{3}\right)$ tal que $\mathrm{L} u=f$, então

$$
c(x) \partial_{x} \hat{u}(x, 0, k)+i k \hat{u}(x, 0, k)=\hat{f}(x, 0, k),
$$

para todo $k>0$ e $x \in \mathbb{T}^{1}$. Em particular, para $x \in\left(x_{\ell}, x_{\ell+1}\right)$, podemos escrever

$$
\hat{u}(x, 0, k)=v_{k}(x)+w_{k}(x),
$$

onde

$$
v_{k}(x)=\exp \left\{-i k \int_{\eta}^{x} \frac{1}{c(s)} d s\right\} \int_{x_{\ell}}^{x} \exp \left\{i k \int_{\eta}^{x^{\prime}} \frac{1}{c(s)} d s\right\} \hat{f}\left(x^{\prime}, 0, k\right) / c\left(x^{\prime}\right) d x^{\prime}
$$

e

$$
w_{k}(x)=C_{k} \exp \left\{-i k \int_{\eta}^{x} \frac{1}{c(s)} d s\right\} \quad\left(C_{k} \in \mathbb{C}\right)
$$

é uma solução geral da equação homogênea $w_{k}^{\prime}(x)+i k \frac{w_{k}}{c}(x)=0, x \in\left(x_{\ell}, x_{\ell+1}\right)$. 
Note que, $v_{k}(x)=0$ para todo $x<\inf \operatorname{supp} \psi_{2}$, uma vez que para tais $x$, temos $\hat{f}(x, 0, k)=0$. Em particular, $\lim _{x \rightarrow x_{\ell}^{+}} v_{k}(x)=0$.

Por outro lado,

$$
\left|\exp \left\{-i k \int_{\eta}^{x} \frac{1}{c(s)} d s\right\}\right|=\exp \left\{-k \int_{\eta}^{x} \frac{b}{a^{2}+b^{2}}(s) d s\right\}
$$

e $2 \leqslant m_{\ell} \leqslant 2 n_{\ell}-1$; donde segue que $\lim _{x \rightarrow x_{\ell}^{+}}\left|w_{k}(x)\right|=\infty$, se $C_{k} \neq 0$. Como $\hat{u}\left(x_{\ell}, 0, k\right)=0$, devemos ter $C_{k}=0$. Assim, $\hat{u}(\cdot, 0, k)=v_{k}$ e, consequentemente,

$$
\begin{gathered}
|\hat{u}(\eta+\epsilon, 0, k)|= \\
\left|\exp \left\{-i k \int_{\eta}^{\eta+\epsilon} \frac{1}{c(s)} d s\right\}\right| e^{-k M}\left|\int_{x_{\ell}}^{\eta+\epsilon} \exp \left\{i k \int_{\eta}^{x^{\prime}} \frac{1}{c(s)}-\frac{a}{a^{2}+b^{2}}(s) d s\right\} \psi_{2}\left(x^{\prime}\right) d x^{\prime}\right|= \\
\exp \left\{-k \int_{\eta}^{\eta+\epsilon} \frac{b}{a^{2}+b^{2}}(s) d s\right\} e^{-k M} \int_{x_{\ell}}^{\eta+\epsilon} \exp \left\{k \int_{\eta}^{x^{\prime}} \frac{b}{a^{2}+b^{2}}(s) d s\right\} \psi_{2}\left(x^{\prime}\right) d x^{\prime} \geqslant \\
\exp \left\{k\left(-\int_{\eta}^{\eta+\epsilon}+\int_{y_{0}}^{\eta+\epsilon}+\int_{\eta}^{y_{0}}\right) \frac{b}{a^{2}+b^{2}}(s) d s\right\}=1,
\end{gathered}
$$

o que é uma contradição, pois $\hat{u}(\cdot, 0, k)$ decai rapidamente.

Em resumo, a demonstração do Teorema 4.8 segue das proposições 4.17, 4.18, 4.19 e do Lema 4.16.

\section{II Demonstração do Teorema 4.10}

Estamos considerando o campo (dado por (4.1))

$$
\mathbf{L}=\frac{\partial}{\partial t}+(a(x)+i b(x))\left(\frac{\partial}{\partial x}+\lambda \frac{\partial}{\partial y}\right)
$$

e ainda estamos sob as condições de que $\lambda$ não é um número de Liouville e $c$ possui apenas zeros de ordem finita, mas agora consideraremos o caso em que

$$
\begin{aligned}
\emptyset \neq c^{-1}(0) & =\left\{x_{1}<\ldots<x_{N}<x_{N+1} \doteq x_{1}+2 \pi\right\} \\
& =\mathrm{V}_{2}=\left\{x_{\ell} \in c^{-1}(0) ; 1 \leqslant n_{\ell}<\infty, 2 n_{\ell}-1<m_{\ell} \leqslant \infty\right\}
\end{aligned}
$$


A demonstração do Teorema 4.10 será dividida nas proposições 4.20, 4.22 e no Lema 4.16. Iniciaremos mostrando que $\mathrm{L}$ não é globalmente resolúvel quando $b$ muda de sinal entre dois zeros consecutivos de $c$.

Proposição 4.20. Suponha que $c^{-1}(0) \neq \emptyset$ e que c possui apenas zeros de ordem finita. Se existe $\ell \in\{1, \ldots, N\}$ tal que $b$ muda de sinal em $\left(x_{\ell}, x_{\ell+1}\right)$ e se $x_{\ell} \in \mathrm{V}_{2}$ ou $x_{\ell+1} \in \mathrm{V}_{2}$, então L não é globalmente resolúvel.

Demonstração: Seguiremos a mesma linha da demonstração da Proposição 4.19.

Vamos construir uma função $f \in\left(\operatorname{ker}^{t} \mathrm{~L}\right)^{\circ} \backslash L \mathcal{C}^{\infty}\left(\mathbb{T}^{3}\right)$, a qual será do tipo

$$
f(x, y, t)=\sum_{k=1}^{\infty} \hat{f}(x, 0, k) e^{i k t}, \quad \hat{f}(\cdot, 0, k) \in \mathcal{C}^{\infty}\left(\mathbb{T}^{1}\right)
$$

onde $\hat{f}(\cdot, j, k)$ denota os coeficientes da série parcial de Fourier com relação às variáveis $(y, t)$.

Como $b$ muda de sinal em $\left(x_{\ell}, x_{\ell+1}\right)$, sem perda de generalidade podemos supor que existem $x_{1}$ e $x_{2}$ em $\left(x_{\ell}, x_{\ell+1}\right)$ tais que $b\left(x_{1}\right)<0<b\left(x_{2}\right)$.

Definindo

$$
\sigma=\inf \left\{x \in\left(x_{1}, x_{2}\right) ; b(s) \geqslant 0, s \in\left(x, x_{2}\right)\right\}
$$

segue que $b \geqslant 0$ em $\left(\sigma, x_{2}\right)$ e que existe uma sequência $\sigma_{n} \rightarrow \sigma$, tal que $\sigma_{n}<\sigma$ e $b\left(\sigma_{n}\right)<0$, para todo $n \in \mathbb{N}$.

Fixemos $\psi_{1} \in \mathcal{C}_{c}^{\infty}\left(\left(\sigma, x_{2}\right)\right)$ de forma que $b>0$ em $\operatorname{supp} \psi_{1}, \psi_{1} \geqslant 0$ e $\int_{x_{\ell}}^{x_{\ell+1}} \psi_{1}=1$. Tomando $n \in \mathbb{N}$ suficientemente grande, encontramos $\eta \doteq \sigma_{n}<\sigma$ tal que $b(\eta)<0$ e $\int_{\eta}^{x} \frac{b}{a^{2}+b^{2}}(s) d s>0$, qualquer que seja $x \in \operatorname{supp} \psi_{1}$. Como $b(\eta)<0$, existe $\epsilon>0$ tal que $b<0$ em $(\eta, \eta+\epsilon)$. Agora considere $\psi_{2} \in \mathcal{C}_{c}^{\infty}((\eta, \eta+\epsilon))$ tal que $\psi_{2} \geqslant 0$ e $\int_{x_{\ell}}^{x_{\ell+1}} \psi_{2}=1$.

O próximo passo antes de definir as funções $\hat{f}(\cdot, 0, k)$ é considerar $y_{0} \doteq \sup \operatorname{supp} \psi_{2}$, $M \doteq-\int_{y_{0}}^{\eta+\epsilon} \frac{b}{a^{2}+b^{2}}(s) d s>0$ e $d_{k}=-\int_{x_{\ell}}^{x_{\ell+1}} \psi_{2}(x) \exp \left\{k \int_{\eta}^{x} \frac{b}{a^{2}+b^{2}}(s) d s\right\} d x$.

Daí, para $k>0$ definimos

$$
\begin{aligned}
& \hat{f}(x, 0, k)= \\
& \quad c(x) e^{-k M}\left[\psi_{2}(x) \exp \left\{-i k \int_{\eta}^{x} \frac{a}{a^{2}+b^{2}}(s) d s\right\}+\psi_{1}(x) \exp \left\{-i k \int_{\eta}^{x} \frac{1}{c(s)} d s\right\} d_{k}\right] .
\end{aligned}
$$


Argumentando como na demonstração da Proposition 4.19, verifica-se que $\hat{f}(\cdot, 0, k)$ decai rapidamente e que a função $f$ definida por (4.44) pertence a $\left(\operatorname{ker}^{t} \mathrm{~L}\right)^{\circ}$.

Finalizando a demonstração, mostraremos que não existe $u \in \mathcal{C}^{\infty}\left(\mathbb{T}^{3}\right)$ solução de $\mathrm{L} u=f$. De fato, se existir, então para todo $k>0$ a série parcial de Fourier implica que $\hat{u}(\cdot, 0, k)$ satisfaz

$$
c(x) \partial_{x} \hat{u}(x, 0, k)+i k \hat{u}(x, 0, k)=\hat{f}(x, 0, k)
$$

e, consequentemente,

$$
\partial_{x} \hat{u}(x, 0, k)+\frac{i k}{c(x)} \hat{u}(x, 0, k)=\frac{\hat{f}(x, 0, k)}{c(x)}
$$

para todo $x \in\left(x_{\ell}, x_{\ell+1}\right)$. Sendo assim, podemos escrever

$$
\hat{u}(x, 0, k)=v_{k}(x)+w_{k}(x),
$$

onde

$$
v_{k}(x)=\exp \left\{-i k \int_{\eta}^{x} \frac{1}{c(s)} d s\right\} \int_{x_{\ell}}^{x} \exp \left\{i k \int_{\eta}^{x^{\prime}} \frac{1}{c(s)} d s\right\} \hat{f}\left(x^{\prime}, 0, k\right) / c\left(x^{\prime}\right) d x^{\prime}
$$

e

$$
w_{k}(x)=C_{k} \exp \left\{-i k \int_{\eta}^{x} \frac{1}{c(s)} d s\right\} \quad\left(C_{k} \in \mathbb{C}\right)
$$

é uma solução geral da equação homogênea $w_{k}^{\prime}(x)+i k \frac{w_{k}}{c}(x)=0, x \in\left(x_{\ell}, x_{\ell+1}\right)$. Note que

$$
w_{k}^{\prime}(x)=-i k \frac{C_{k}}{c(x)} \exp \left\{-i k \int_{\eta}^{x} \frac{1}{c(s)} d s\right\}, x \in\left(x_{\ell}, x_{\ell+1}\right) .
$$

Se $x_{\ell} \in \mathrm{V}_{2}$ (ou seja, $n_{\ell}<\infty$ e $m_{\ell}>2 n_{\ell}-1$ ), então podemos escrever $\frac{b}{a^{2}+b^{2}}\left(x^{\prime}\right)=$ $\left(x^{\prime}-x_{\ell}\right)^{\rho} \alpha\left(x^{\prime}\right)$, para $x^{\prime} \in\left[x_{\ell}, x_{\ell}+\delta\right)$, onde $\rho \geqslant 0$. Desta forma, $b /\left(a^{2}+b^{2}\right)$ pertence a $\mathrm{L}^{1}\left(\left[x_{\ell}, \eta\right]\right)$; logo, $\lim _{x \rightarrow x_{\ell}^{+}}\left|w_{k}^{\prime}(x)\right|=\infty$, se $C_{k} \neq 0$. Analogamente, $x_{\ell+1} \in \mathrm{V}_{2}$ implica que $\lim _{x \rightarrow x_{\ell+1}^{-}}\left|w_{k}^{\prime}(x)\right|=\infty$, se $C_{k} \neq 0$. Por outro lado, $v_{k} \equiv 0$ em uma vizinhança de $x_{\ell}$, uma vez que $\hat{f}(x, 0, k)=0$ para todo $x<\inf \operatorname{supp} \psi_{2}$. Da mesma forma, para todo $x>\sup \operatorname{supp} \psi_{1}$, temos 


$$
\begin{gathered}
\int_{x_{\ell}}^{x} \exp \left\{i k \int_{\eta}^{x^{\prime}} \frac{1}{c(s)} d s\right\} \hat{f}\left(x^{\prime}, 0, k\right) / c\left(x^{\prime}\right) d x^{\prime}= \\
e^{-k M} \int_{x_{\ell}}^{x_{\ell}+1} \exp \left\{i k \int_{\eta}^{x^{\prime}} \frac{1}{c(s)} d s\right\} \psi_{2}\left(x^{\prime}\right) \exp \left\{-i k \int_{\eta}^{x^{\prime}} \frac{a}{a^{2}+b^{2}}(s) d s\right\} d x^{\prime}+ \\
e^{-k M} \int_{x_{\ell}}^{x_{\ell}+1} d_{k} \exp \left\{i k \int_{\eta}^{x^{\prime}} \frac{1}{c(s)} d s\right\} \psi_{1}\left(x^{\prime}\right) \exp \left\{-i k \int_{\eta}^{x^{\prime}} \frac{1}{c(s)} d s\right\} d x^{\prime}= \\
e^{-k M}\left(\int_{x_{\ell}}^{x_{\ell}+1} \psi_{2}\left(x^{\prime}\right) \exp \left\{k \int_{\eta}^{x^{\prime}} \frac{b}{a^{2}+b^{2}}(s) d s\right\} d x^{\prime}+d_{k}\right)=0
\end{gathered}
$$

$\operatorname{logo}, v_{k}(x)=0$ para todo $x>\sup \operatorname{supp} \psi_{1}$. Assim, devemos ter $C_{k}=0$. Consequentemente,

$$
\begin{gathered}
|\hat{u}(\eta+\epsilon, 0, k)|=\left|v_{k}(\eta+\epsilon)\right|= \\
\left|\exp \left\{-i k \int_{\eta}^{\eta+\epsilon} \frac{1}{c(s)} d s\right\}\right| e^{-k M}\left|\int_{x_{\ell}}^{\eta+\epsilon} \exp \left\{i k \int_{\eta}^{x^{\prime}} \frac{1}{c(s)}-\frac{a}{a^{2}+b^{2}}(s) d s\right\} \psi_{2}\left(x^{\prime}\right) d x^{\prime}\right|= \\
\exp \left\{-k \int_{\eta}^{\eta+\epsilon} \frac{b}{a^{2}+b^{2}}(s) d s\right\} e^{-k M} \int_{x_{\ell}}^{\eta+\epsilon} \exp \left\{k \int_{\eta}^{x^{\prime}} \frac{b}{a^{2}+b^{2}}(s) d s\right\} \psi_{2}\left(x^{\prime}\right) d x^{\prime} \geqslant \\
\exp \left\{k\left(-\int_{\eta}^{\eta+\epsilon}+\int_{y_{0}}^{\eta+\epsilon}+\int_{\eta}^{y_{0}}\right) \frac{b}{a^{2}+b^{2}}(s) d s\right\}=1,
\end{gathered}
$$

o que é uma contradição, pois $\hat{u}(\cdot, 0, k)$ decai rapidamente.

A próxima proposição mostra que as condições dadas no Teorema 4.10 implicam a resolubilidade global de $\mathrm{L}$. Para cada $f \in\left(\operatorname{ker}^{t} \mathrm{~L}\right)^{\circ}$, mostraremos que existe $u \in \mathcal{C}^{\infty}\left(\mathbb{T}^{3}\right)$ tal que $\mathrm{L} u=f$, sendo que, pelo Lema 4.16 podemos supor que $f$ é flat em $c^{-1}(0) \times \mathbb{T}^{2}$, como na seção anterior. Para completar tal objetivo, utilizaremos o seguinte resultado auxiliar.

Lema 4.21. Suponha que c possui apenas zeros de ordem finita e sejam $x_{\ell}$ e $x_{\ell+1}$ dois zeros consecutivos de c. Fixado $\eta \in\left(x_{\ell}, x_{\ell+1}\right)$, defina

$$
\theta_{j k}(x) \doteq \int_{\eta}^{x}\left(\frac{i k}{c(s)}+i \lambda j\right) d s, \quad \text { para todo } x \in\left(x_{\ell}, x_{\ell+1}\right), \text { onde } j \in \mathbb{Z} \text { e } k \in \mathbb{Z} \backslash\{0\}
$$

Suponha que a função $\exp \left\{\theta_{j k}\right\}$ seja limitada em $\left(x_{\ell}, x_{\ell+1}\right)$. Além disso, suponha que $m_{j} \geqslant 2$ ou $m_{j}=n_{j}=1$, para $j=\ell, \ell+1$. Nestas condições, para cada $f \in\left(\operatorname{ker}^{t} \mathrm{~L}\right)^{\circ}$, 
flat em $c^{-1}(0) \times \mathbb{T}^{2}$, temos

$$
\int_{x_{\ell}}^{x_{\ell+1}} \exp \left\{\theta_{j k}(x)\right\} \hat{f}(x, j, k) / c(x) d x=0
$$

onde $\hat{f}(\cdot, j, k)$ denota o coeficiente parcial de Fourier com relação às variáveis $(y, t)$.

Demonstração: Como em [BP], a identidade (4.45) será uma consequência do fato de que $f$ pertence a $\left(\operatorname{ker}^{t} \mathrm{~L}\right)^{\circ}$. Iremos encontrar certas distribuições em $\operatorname{ker}^{t} \mathrm{~L}$, as quais produzirão (4.45).

Note que $\theta_{j k} \in \mathcal{C}^{\infty}\left(\left(x_{\ell}, x_{\ell+1}\right)\right)$ e por hipótese $\exp \left\{\theta_{j k}\right\}$ é limitada em $\left(x_{\ell}, x_{\ell+1}\right)$. Defina

$$
\psi_{j k}(x)=\left\{\begin{array}{l}
\exp \left\{\theta_{j k}(x)\right\}, \text { se } x \in\left(x_{\ell}, x_{\ell+1}\right) \\
0, \text { se } x \in \mathbb{T}^{1} \backslash\left(x_{\ell}, x_{\ell+1}\right),
\end{array}\right.
$$

A função $\psi_{j k}$ está em $\mathrm{L}^{\infty}\left(\mathbb{T}^{1}\right) \subset \mathcal{D}^{\prime}\left(\mathbb{T}^{1}\right)$. Como $c$ possui apenas anulamentos de ordem finita, existe $\psi_{j k} / c \in \mathcal{D}^{\prime}\left(\mathbb{T}^{1}\right)$ tal que $c\left(\psi_{j k} / c\right)=\psi_{j k} \operatorname{esupp}\left(\psi_{j k} / c\right) \subset \operatorname{supp} \psi_{j k}$. Definindo $\omega_{j k}=\psi_{j k}^{\prime}-i k \psi_{j k} / c-i \lambda j \psi_{j k}$, obtemos supp $\omega_{j k} \subset \operatorname{supp} \psi_{j k} \subset\left[x_{\ell}, x_{\ell+1}\right]$. Mais ainda, para toda $\phi \in \mathcal{C}^{\infty}\left(\mathbb{T}^{1}\right)$ tal que $\operatorname{supp} \phi \subset\left(x_{\ell}, x_{\ell+1}\right)$, temos

$$
\left\langle\omega_{j k}, \phi\right\rangle=-\left\langle\psi_{j k}, \phi^{\prime}\right\rangle-i k\left\langle\psi_{j k}, \phi / c\right\rangle-i \lambda j\left\langle\psi_{j k}, \phi\right\rangle
$$

e como

$$
\begin{gathered}
-\left\langle\psi_{j k}, \phi^{\prime}\right\rangle=-\int_{x_{\ell}}^{x_{\ell+1}} \phi^{\prime}(x) \exp \left\{\int_{\eta}^{x}(i k / c(s)+i \lambda j) d s\right\} d x= \\
\int_{x_{\ell}}^{x_{\ell+1}}(i k / c(x)+i \lambda j) \phi(x) \exp \left\{\int_{\eta}^{x}(i k / c(s)+i \lambda j) d s\right\} d x= \\
i k\left\langle\psi_{j k}, \phi / c\right\rangle+i \lambda j\left\langle\psi_{j k}, \phi\right\rangle,
\end{gathered}
$$

obtemos $\left\langle\omega_{j k}, \phi\right\rangle=0$. Assim, $\operatorname{supp} \omega_{j k} \subset\left\{x_{\ell}, x_{\ell+1}\right\}$.

Afirmamos que, para cada $(j, k)$, existe uma distribuição $\mu_{j k}$, também com suporte contido em $\left\{x_{\ell}, x_{\ell+1}\right\}$, tal que $\left(c \mu_{j k}\right)^{\prime}-i k \mu_{j k}-i \lambda j c \mu_{j k}=\omega_{j k}$. De fato, temos

$$
\omega_{j k}=\sum_{m=0}^{r_{\ell}} \alpha_{m} \delta^{(m)}\left(x-x_{\ell}\right)+\sum_{m=0}^{r_{\ell+1}} \beta_{m} \delta^{(m)}\left(x-x_{\ell+1}\right)
$$

e vamos procurar por $\mu_{j k}$ do mesmo tipo 


$$
\sum_{m=0}^{r_{\ell}} \tau_{m} \delta^{(m)}\left(x-x_{\ell}\right)+\sum_{m=0}^{r_{\ell+1}} \gamma_{m} \delta^{(m)}\left(x-x_{\ell+1}\right) .
$$

É suficiente resolver as seguintes equações:

$$
\begin{gathered}
\sum_{m=0}^{r_{\ell}} \alpha_{m} \delta^{(m)}\left(x-x_{\ell}\right)=\sum_{m=1}^{r_{\ell}} \sum_{n=1}^{m} \tau_{m}\left(\begin{array}{c}
m \\
n
\end{array}\right)(-1)^{n} c^{(n)}\left(x_{\ell}\right) \delta^{(m-n+1)}\left(x-x_{\ell}\right)- \\
i k \sum_{m=0}^{r_{\ell+1}} \tau_{m} \delta^{(m)}\left(x-x_{\ell}\right)-i \lambda j \sum_{m=1}^{r_{\ell}} \sum_{n=1}^{m} \tau_{m}\left(\begin{array}{c}
m \\
n
\end{array}\right)(-1)^{n} c^{(n)}\left(x_{\ell}\right) \delta^{(m-n)}\left(x-x_{\ell}\right)
\end{gathered}
$$

e

$$
\begin{gathered}
\sum_{m=0}^{r_{\ell+1}} \beta_{m} \delta^{(m)}\left(x-x_{\ell+1}\right)=\sum_{m=1}^{r_{\ell+1}} \sum_{n=1}^{m} \gamma_{m}\left(\begin{array}{c}
m \\
n
\end{array}\right)(-1)^{n} c^{(n)}\left(x_{\ell+1}\right) \delta^{(m-n+1)}\left(x-x_{\ell+1}\right)- \\
i k \sum_{m=0}^{r_{\ell+1}} \gamma_{m} \delta^{(m)}\left(x-x_{\ell+1}\right)-i \lambda j \sum_{m=1}^{r_{\ell+1}} \sum_{n=1}^{m} \gamma_{m}\left(\begin{array}{c}
m \\
n
\end{array}\right)(-1)^{n} c^{(n)}\left(x_{\ell+1}\right) \delta^{(m-n)}\left(x-x_{\ell+1}\right) .
\end{gathered}
$$

Vamos resolver (4.47). Defina $C_{m, n}=\left(\begin{array}{c}m \\ n\end{array}\right)(-1)^{n}$ e note que

$$
\begin{gathered}
\sum_{m=1}^{r_{\ell}} \sum_{n=1}^{m} \tau_{m}\left(\begin{array}{c}
m \\
n
\end{array}\right)(-1)^{n} c^{(n)}\left(x_{\ell}\right) \delta^{(m-n)}\left(x-x_{\ell}\right)= \\
\sum_{m=1}^{r_{\ell}}\left(\sum_{n=m}^{r_{\ell}} \tau_{n} C_{n, n-m+1} c^{(n-m+1)}\left(x_{\ell}\right)\right) \delta^{(m-1)}\left(x-x_{\ell}\right) .
\end{gathered}
$$

A identidade acima implica que basta resolver as seguintes equações:

$$
\begin{gathered}
\alpha_{r_{\ell}}=\left(-i k+C_{r_{\ell}, 1} c^{\prime}\left(x_{\ell}\right)\right) \tau_{r_{\ell}}, \\
\alpha_{r_{\ell}-1}=\left(-i k+C_{r_{\ell}-1,1} c^{\prime}\left(x_{\ell}\right)\right) \tau_{r_{\ell}-1}-i \lambda j \tau_{r_{\ell}} C_{r_{\ell}, 1} c^{\prime}\left(x_{\ell}\right)+\tau_{r_{\ell}} C_{r_{\ell}, 2} c^{(2)}\left(x_{\ell}\right), \\
\alpha_{m}=\left(-i k+C_{m, 1} c^{\prime}\left(x_{\ell}\right)\right) \tau_{m}+\sum_{n=m+1}^{r_{\ell}}\left[-i \lambda j C_{n, n-m} c^{(n-m)}\left(x_{\ell}\right)+C_{n, n-m+1} c^{(n-m+1)}\left(x_{\ell}\right)\right] \tau_{n}
\end{gathered}
$$

$\left(\right.$ para $\left.0<m<r_{\ell}\right)$ e

$$
\alpha_{0}=-i k \tau_{0}-i \lambda j \sum_{n=1}^{r_{\ell}} \tau_{n} C_{n, n} c^{(n)}\left(x_{\ell}\right) .
$$


Como $k \in \mathbb{Z} \backslash\{0\}, C_{n, m} \in \mathbb{R} \backslash\{0\}$ e como $m_{\ell} \geqslant 2$ ou $m_{\ell}=n_{\ell}=1$ (por hipótese), segue que podemos determinar sucessivamente $\tau_{r_{\ell}}, \tau_{r_{\ell}-1}, \ldots, \tau_{1}, \tau_{0}$ de modo que (4.47) se verifique.

Analogamente, determinamos $\gamma_{r_{\ell+1}}, \gamma_{r_{\ell+1}-1}, \ldots, \gamma_{1}, \gamma_{0}$ de modo que valha (4.48).

Portanto, obtemos

$$
\mu_{j k}=\sum_{m=0}^{r_{\ell}} \tau_{m} \delta^{(m)}\left(x-x_{\ell}\right)+\sum_{m=0}^{r_{\ell+1}} \gamma_{m} \delta^{(m)}\left(x-x_{\ell+1}\right)
$$

tal que $\left(c \mu_{j k}\right)^{\prime}-i k \mu_{j k}-i \lambda j c \mu_{j k}=\omega_{j k}=\psi_{j k}^{\prime}-i k \psi_{j k} / c-i \lambda j \psi_{j k}$. Em particular, $\nu_{j k}=$ $\psi_{j k} / c-\mu_{j k}$ satisfaz $\left(c \nu_{j k}\right)^{\prime}-i k \nu_{j k}-i \lambda j c \nu_{j k}=0$; assim, $\nu_{j k} \otimes e^{-i(j y+k t)}$ pertence a ker ${ }^{t} \mathbf{L}$. Consequentemente,

$0=\left\langle\nu_{j k} \otimes e^{-i(j y+k t)}, f\right\rangle=(2 \pi)^{2}\left\langle\nu_{j k}, \hat{f}(\cdot, j, k)\right\rangle=(2 \pi)^{2}\left[\left\langle\psi_{j k} / c, \hat{f}(\cdot, j, k)\right\rangle-\left\langle\mu_{j k}, \hat{f}(\cdot, j, k)\right\rangle\right]$.

Como $f$ é flat em $c^{-1}(0)$, segue que $\left\langle\mu_{j k}, \hat{f}(\cdot, j, k)\right\rangle=0$, logo

$$
0=\left\langle\psi_{j k} / c, \hat{f}(\cdot, j, k)\right\rangle=\int_{x_{\ell}}^{x_{\ell+1}} \exp \left\{\theta_{j k}(x)\right\} \hat{f}(x, j, k) / c(x) d x .
$$

O que conclui a demonstração do Lema 4.21.

Proposição 4.22. Suponha que $\lambda$ não é um número de Liouville e que $\emptyset \neq c^{-1}(0)=$ $\left\{x_{1}<\cdots<x_{N}<x_{N+1} \doteq x_{1}+2 \pi\right\}=\mathrm{V}_{2}$, como em (4.43). Suponha também que $b$ não muda de sinal em $\left(x_{\ell}, x_{\ell+1}\right)$ e que existe $\delta>0$ tal que $b \equiv 0$ em $\left(x_{\ell}, x_{\ell}+\delta\right) \cup$ $\left(x_{\ell+1}-\delta, x_{\ell+1}\right)$, para cada $\ell=1, \ldots, N$. Neste caso, para cada $f \in\left(\operatorname{ker}^{t} \mathrm{~L}\right)^{\circ}$ que é flat em $c^{-1}(0) \times \mathbb{T}^{2}$ existe $u \in \mathcal{C}^{\infty}\left(\mathbb{T}^{3}\right)$ tal que $\mathrm{L} u=f$.

Demonstração: Seja $f \in\left(\operatorname{ker}^{t} \mathrm{~L}\right)^{\circ}, f$ flat em $c^{-1}(0)$. Utilizando série parcial de Fourier com relação às variáveis $(y, t)$, segue que uma função $u \in \mathcal{C}^{\infty}\left(\mathbb{T}^{3}\right)$ satisfaz $L u=f$ se, e somente se,

$$
c(x) \partial_{x} \hat{u}(x, j, k)+i(k+c(x) \lambda j) \hat{u}(x, j, k)=\hat{f}(x, j, k),
$$

quaisquer que sejam $x \in \mathbb{T}^{1}$ e $(j, k) \in \mathbb{Z}^{2}$.

Para $k=0$, procedemos como na demonstração da Proposição 4.17 de modo que obtemos uma sequência rapidamente decrescente $\hat{u}(\cdot, j, 0)$, cujos termos satisfazem (4.49).

Quando $k \in \mathbb{Z} \backslash\{0\}$ e $j \in \mathbb{Z}$, mostraremos que (4.49) tem uma solução em $\left[x_{\ell}, x_{\ell+1}\right]$, a qual é flat em $\left\{x_{\ell}, x_{\ell+1}\right\}$, para cada $\ell=1, \ldots, N$. Mais ainda, colando tais soluções 
obteremos uma sequência rapidamente decrescente $\hat{u}(\cdot, j, k)(k \neq 0)$, cujos termos são funções em $\mathcal{C}^{\infty}\left(\mathbb{T}^{1}\right)$ que satisfazem (4.49).

Note que, no intervalo $\left(x_{\ell}, x_{\ell+1}\right)$ a equação (4.49) torna-se

$$
\partial_{x} \hat{u}(x, j, k)+i(k / c(x)+\lambda j) \hat{u}(x, j, k)=\hat{f}(x, j, k) / c(x),
$$

para todo $(j, k) \in \mathbb{Z} \times(\mathbb{Z} \backslash\{0\})$.

Fixado $\eta \in\left(x_{\ell}, x_{\ell+1}\right)$, considere

$$
\theta_{j k}(x)=i \int_{\eta}^{x}(k / c(s)+\lambda j) d s=\int_{\eta}^{x}\left(k \frac{b+i a}{a^{2}+b^{2}}(s)+i \lambda j\right) d s, x \in\left(x_{\ell}, x_{\ell+1}\right) .
$$

A hipótese $\left\{x_{\ell}, x_{\ell+1}\right\} \in \mathrm{V}_{2}$ implica que $\frac{b}{a^{2}+b^{2}} \in \mathrm{L}^{1}\left(x_{\ell}, x_{\ell+1}\right) ; \operatorname{assim}, \exp \left\{ \pm \theta_{j k}\right\}$ é limitada em $\left(x_{\ell}, x_{\ell+1}\right)$ e podemos definir a função

$$
\begin{aligned}
\hat{u}(x, j, k) & =\exp \left\{-\theta_{j k}(x)\right\} \int_{x_{\ell}}^{x} \exp \left\{\theta_{j k}\left(x^{\prime}\right)\right\} \hat{f}\left(x^{\prime}, j, k\right) / c\left(x^{\prime}\right) d x^{\prime} \\
& =\int_{x_{\ell}}^{x} \exp \left\{-k \int_{x^{\prime}}^{x} \frac{b+i a}{a^{2}+b^{2}}(s) d s+i \lambda j\left(x^{\prime}-x\right)\right\} \hat{f}\left(x^{\prime}, j, k\right) / c\left(x^{\prime}\right) d x^{\prime},
\end{aligned}
$$

para todo $x \in\left(x_{\ell}, x_{\ell+1}\right)$, a qual é uma solução suave de (4.50) no intervalo $\left(x_{\ell}, x_{\ell+1}\right)$. Mais ainda, como $\hat{f}(\cdot, j, k) / c$ é flat em $x_{\ell}$ e como $\exp \left\{ \pm \theta_{j k}\right\}$ é limitada em $\left(x_{\ell}, x_{\ell+1}\right)$, segue que ambas as funções $\exp \left\{\theta_{j k}\right\} \hat{f}(\cdot, j, k) / c$ e $\hat{u}(\cdot, j, k)$ são flat em $x_{\ell}$.

Analogamente, a função $\exp \left\{\theta_{j k}\right\} \hat{f}(\cdot, j, k) / c$ é flat em $x_{\ell+1}$. Assim, para que a solução $\hat{u}(\cdot, j, k)$ seja flat em $x_{\ell+1}$ basta que

$$
\int_{x_{\ell}}^{x_{\ell+1}} \exp \left\{\theta_{j k}(x)\right\} \hat{f}(x, j, k) / c(x) d x=0, \quad(j, k) \in \mathbb{Z} \times(\mathbb{Z} \backslash\{0\}) .
$$

Tal identidade segue ao aplicarmos o Lema 4.21.

Portanto, $\hat{u}(\cdot, j, k)(k \neq 0)$ é uma solução suave de (4.50), a qual é flat em $\left\{x_{\ell}, x_{\ell+1}\right\}$.

Como $\ell$ foi fixado arbitrariamente em $\{1, \ldots, N\}$, obtemos uma função $\hat{u}(\cdot, j, k) \in$ $\mathcal{C}^{\infty}\left(\mathbb{T}^{1}\right)$, a qual é solução de (4.49) e flat em $c^{-1}(0)$.

Para finalizar a demonstração, precisamos mostrar que a sequência $\hat{u}(\cdot, j, k) \subset \mathcal{C}^{\infty}\left(\mathbb{T}^{1}\right)$ é rapidamente decrescente, onde $(j, k) \in \mathbb{Z} \times(\mathbb{Z} \backslash\{0\})$. Para tanto, como na demonstração da Proposição 4.17, é suficiente provar que para cada $\ell \in\{1, \ldots, N\}$ e para 
quaisquer $\gamma_{1} \in \mathbb{Z}_{+}, \gamma_{2} \in \mathbb{Z}_{+}$e $\gamma_{3} \in \mathbb{Z}_{+}$, existe $C=C\left(\gamma_{1}, \gamma_{2}, \gamma_{3}, \ell\right)>0$ tal que

$$
|j|^{\gamma_{1}}|k|^{\gamma_{2}}|c(x)|^{-\gamma_{3}}|\hat{u}(x, j, k)| \leqslant C
$$

para todo $x \in\left(x_{\ell}, x_{\ell+1}\right)$ e para todo $(j, k) \in \mathbb{Z} \times(\mathbb{Z} \backslash\{0\})$.

Definindo $g=f / c \in \mathcal{C}^{\infty}\left(\mathbb{T}^{3}\right)$, obtemos $\hat{g}(\cdot, j, k)=\hat{f}(\cdot, j, k) / c$ e como $j^{\gamma_{1}} k^{\gamma_{2}} \hat{g}(\cdot, j, k)=$ $(-1)^{\gamma_{1}+\gamma_{2}}\left(\partial^{\left(0, \gamma_{1}, \gamma_{2}\right)} g\right) \hat{(}(\cdot, j, k)$, da definição de $\hat{u}(\cdot, j, k)$ segue que

$$
\begin{gathered}
|j|^{\gamma_{1}}|k|^{\gamma_{2}}|c(x)|^{-\gamma_{3}}|\hat{u}(x, j, k)|= \\
\left.|c(x)|^{-\gamma_{3}} \mid \int_{x_{\ell}}^{x} \exp \left\{-k \int_{x^{\prime}}^{x} \frac{b+i a}{a^{2}+b^{2}}(s) d s+i \lambda j\left(x^{\prime}-x\right)\right\}\left(\partial^{\left(0, \gamma_{1}, \gamma_{2}\right)} g\right) \hat{(} x^{\prime}, j, k\right) d x^{\prime} \mid .
\end{gathered}
$$

Vale lembrar que, por hipótese, $b \equiv 0$ em $\left(x_{\ell}, x_{\ell}+\delta\right) \cup\left(x_{\ell+1}-\delta, x_{\ell+1}\right)$ e que $b$ não muda de sinal em $\left(x_{\ell}, x_{\ell+1}\right)$. Sendo assim, de (4.54) segue que, para $x \in\left(x_{\ell}, x_{\ell}+\delta\right)$, temos

$$
\begin{gathered}
\left.|j|^{\gamma_{1}}|k|^{\gamma_{2}}|c(x)|^{-\gamma_{3}}|\hat{u}(x, j, k)| \leqslant|c(x)|^{-\gamma_{3}} \int_{x_{\ell}}^{x} \mid\left(\partial^{\left(0, \gamma_{1}, \gamma_{2}\right)} g\right) \hat{(} x^{\prime}, j, k\right) \mid d x^{\prime} \leqslant \\
|c(x)|^{-\gamma_{3}}\left|x_{\ell+1}-x_{\ell}\right| \sup _{x^{\prime} \in\left[x_{\ell}, x\right]}\left\{\left|\left(\partial^{\left(0, \gamma_{1}, \gamma_{2}\right)} g\right) \hat{(}\left(x^{\prime}, j, k\right)\right|\right\} \leqslant \\
|c(x)|^{-\gamma_{3}}\left|x_{\ell+1}-x_{\ell}\right| \sup \left\{\left|\left(\partial^{\left(0, \gamma_{1}, \gamma_{2}\right)} g\right)\left(x^{\prime}, y, t\right)\right| ;\left(x^{\prime}, y, t\right) \in\left(x_{\ell}, x\right) \times \mathbb{T}^{2}\right\} .
\end{gathered}
$$

Mais ainda, como $g=f / c$ é flat em $c^{-1}(0) \times \mathbb{T}^{2}$, obtemos uma constante $C_{1}>0$ tal que

$$
|j|^{\gamma_{1}}|k|^{\gamma_{2}}|c(x)|^{-\gamma_{3}}|\hat{u}(x, j, k)| \leqslant C_{1}
$$

para todo $x \in\left[x_{\ell}, x_{\ell}+\delta\right]$ e $(j, k) \in \mathbb{Z} \times(\mathbb{Z} \backslash\{0\})$.

Para $x \in\left[x_{\ell}+\delta, x_{\ell+1}-\delta\right]$ e para $k \in \mathbb{Z} \backslash\{0\}$ tal que $k b \geqslant 0$ em $\left[x_{\ell}+\delta, x_{\ell+1}-\delta\right]$, utilizando (4.54) obtemos

$$
\begin{aligned}
|j|^{\gamma_{1}}|k|^{\gamma_{2}}|c(x)|^{-\gamma_{3}}|\hat{u}(x, j, k)| & \left.\leqslant\left(\sup _{x \in\left[x_{\ell}+\delta, x_{\ell+1}-\delta\right]}|c(x)|^{-\gamma_{3}}\right)\left|x_{\ell+1}-x_{\ell}\right| \cdot \|\left(\partial^{\left(0, \gamma_{1}, \gamma_{2}\right)} g\right) \hat{(} \cdot, j, k\right) \|_{\infty} \\
& \leqslant\left(\sup _{x \in\left[x_{\ell}+\delta, x_{\ell+1}-\delta\right]}|c(x)|^{-\gamma_{3}}\right)\left|x_{\ell+1}-x_{\ell}\right| \cdot\left\|\partial^{\left(0, \gamma_{1}, \gamma_{2}\right)} g\right\|_{\infty}=C_{2},
\end{aligned}
$$

onde $C_{2}>0$ não depende de $(j, k)$. 
Quando $k \in \mathbb{Z} \backslash\{0\}$ é tal que $k b \leqslant 0$ em $\left[x_{\ell}+\delta, x_{\ell+1}-\delta\right]$, utilizamos a identidade (4.53) para escrever

$$
\hat{u}(x, j, k)=-\exp \left\{-\theta_{j k}(x)\right\} \int_{x}^{x_{\ell+1}} \exp \left\{\theta_{j k}\left(x^{\prime}\right)\right\} \hat{f}\left(x^{\prime}, j, k\right) / c\left(x^{\prime}\right) d x^{\prime} .
$$

Desta forma, obtemos

$$
\begin{gathered}
|j|^{\gamma_{1}}|k|^{\gamma_{2}}|c(x)|^{-\gamma_{3}}|\hat{u}(x, j, k)|= \\
|c(x)|^{-\gamma_{3}}\left|\int_{x}^{x_{\ell+1}} \exp \left\{-k \int_{x^{\prime}}^{x} \frac{b+i a}{a^{2}+b^{2}}(s) d s+i \lambda j\left(x^{\prime}-x\right)\right\}\left(\partial^{\left(0, \gamma_{1}, \gamma_{2}\right)} g\right) \hat{(}\left(x^{\prime}, j, k\right) d x^{\prime}\right| .
\end{gathered}
$$

Da identidade acima segue que, para todo $x \in\left[x_{\ell}+\delta, x_{\ell+1}-\delta\right]$ e para $k$ tal que $k b \leqslant 0$, temos

$$
\begin{aligned}
|j|^{\gamma_{1}}|k|^{\gamma_{2}}|c(x)|^{-\gamma_{3}}|\hat{u}(x, j, k)| & \leqslant\left(\sup _{x \in\left[x_{\ell}+\delta, x_{\ell+1}-\delta\right]}|c(x)|^{-\gamma_{3}}\right)\left|x_{\ell+1}-x_{\ell}\right| \cdot\left\|\left(\partial^{\left(0, \gamma_{1}, \gamma_{2}\right)} g\right) \hat{)}(\cdot, j, k)\right\|_{\infty} \\
& \leqslant\left(\sup _{x \in\left[x_{\ell}+\delta, x_{\ell+1}-\delta\right]}|c(x)|^{-\gamma_{3}}\right)\left|x_{\ell+1}-x_{\ell}\right| \cdot\left\|\partial^{\left(0, \gamma_{1}, \gamma_{2}\right)} g\right\|_{\infty}=C_{2},
\end{aligned}
$$

onde $C_{2}>0$ não depende de $(j, k)$.

Por fim, para $x \in\left[x_{\ell+1}-\delta, x_{\ell+1}\right)$ também uilizamos a identidade (4.56) e procedendo como em (4.55) obtemos uma constante $C_{3}>0$ tal que

$$
|j|^{\gamma_{1}}|k|^{\gamma_{2}}|c(x)|^{-\gamma_{3}}|\hat{u}(x, j, k)| \leqslant C_{3}
$$

para todo $(j, k) \in \mathbb{Z} \times(\mathbb{Z} \backslash\{0\})$ e $x \in\left[x_{\ell+1}-\delta, x_{\ell+1}\right]$.

Portanto, a sequência $u(\cdot, j, k)$ decai rapidamente. O que completa a demonstração da Proposição 4.22 .

Resumindo, as proposições 4.20, 4.22 e o Lema 4.16 demonstram o Teorema 4.10.

\section{III Demonstração do Teorema 4.7}

Apresentaremos nesta seção a demonstração do Teorema 4.7, onde consideramos o campo (dado por (4.1))

$$
\mathbf{L}=\frac{\partial}{\partial t}+(a(x)+i b(x))\left(\frac{\partial}{\partial x}+\lambda \frac{\partial}{\partial y}\right)
$$


sendo que $\lambda$ não é um número de Liouville, $c$ possui apenas zeros de ordem finita e

$$
\emptyset \neq c^{-1}(0)=\left\{x_{1}<\ldots<x_{N}<x_{N+1} \doteq x_{1}+2 \pi\right\}=\mathrm{V}_{1} \cup \mathrm{V}_{2},
$$

onde

$$
\mathrm{V}_{1}=\left\{x_{\ell} \in c^{-1}(0) ; 2 \leqslant m_{\ell}<2 n_{\ell}-1, m_{\ell}<\infty, 1<n_{\ell} \leqslant \infty\right\}
$$

e

$$
\mathrm{V}_{2}=\left\{x_{\ell} \in c^{-1}(0) ; 1 \leqslant n_{\ell}<\infty, 2 n_{\ell}-1<m_{\ell} \leqslant \infty\right\}
$$

As demonstrações dos teoremas 4.8 e 4.10 (exibidas nas seções 4.I e 4.II, respectivamente) já contemplam alguns casos do Teorema 4.7. Mais especificamente, das proposições 4.19 e 4.20 segue que L não é globalmente resolúvel quando ocorre (V) ou (VI) no Teorema 4.7.

Verificaremos agora que o operador L é globalmente resolúvel quando, para cada par de zeros consecutivos $\left\{x_{\ell}, x_{\ell+1}\right\}$, ocorre uma das seguintes condições:

(I) $\left\{x_{\ell}, x_{\ell+1}\right\} \subset \mathrm{V}_{1}$ e existe $\eta \in\left(x_{\ell}, x_{\ell+1}\right)$ de modo que $b$ não muda de sinal nos intervalos $\left(x_{\ell}, \eta\right)$ e $\left(\eta, x_{\ell+1}\right)$;

(II) $\left\{x_{\ell}, x_{\ell+1}\right\} \subset \mathrm{V}_{2}, b$ não muda de sinal em $\left(x_{\ell}, x_{\ell+1}\right)$ e existe $\delta>0$ tal que $b \equiv 0$ nos intervalos $\left(x_{\ell}, x_{\ell}+\delta\right)$ e $\left(x_{\ell+1}-\delta, x_{\ell+1}\right)$;

(III) $x_{\ell} \in \mathrm{V}_{1}, x_{\ell+1} \in \mathrm{V}_{2}, b$ não muda de sinal em $\left(x_{\ell}, x_{\ell+1}\right)$ e $b \equiv 0$ em $\left(x_{\ell+1}-\delta, x_{\ell+1}\right)$;

(IV) $x_{\ell} \in \mathrm{V}_{2}, x_{\ell+1} \in \mathrm{V}_{1}, b$ não muda de sinal em $\left(x_{\ell}, x_{\ell+1}\right)$ e $b \equiv 0$ em $\left(x_{\ell}, x_{\ell}+\delta\right)$.

Seja $f \in\left(\operatorname{ker}^{t} \mathrm{~L}\right)^{\circ}$. Precisamos mostrar que $f \in \mathrm{LC} \mathcal{C}^{\infty}\left(\mathbb{T}^{3}\right)$. Como nas seções 4.I e 4.II, do Lema 4.16 segue que podemos supor que $f$ é flat em $c^{-1}(0)$. Para encontrar $u \in \mathcal{C}^{\infty}\left(\mathbb{T}^{3}\right)$ tal que $\mathrm{L} u=f$, vamos escrever $u$ e $f$ por meio da série parcial de Fourier com relação às variáveis $(y, t)$. Assim, obtemos

$$
\begin{aligned}
& u(x, y, t)=\sum_{(j, k) \in \mathbb{Z}^{2}} \hat{u}(x, j, k) e^{i(j y+k t)}, \\
& f(x, y, t)=\sum_{(j, k) \in \mathbb{Z}^{2}} \hat{f}(x, j, k) e^{i(j y+k t)}
\end{aligned}
$$


e $\mathbf{L} u=f$ se, e somente se,

$$
c(x) \partial_{x} \hat{u}(x, j, k)+i(k+\lambda j c(x)) \hat{u}(x, j, k)=\hat{f}(x, j, k),
$$

quaisquer que sejam $x \in \mathbb{T}^{1}$ e $(j, k) \in \mathbb{Z}^{2}$.

Nosso objetivo é encontrar uma sequência rapidamente decrescente $\hat{u}(\cdot, j, k) \subset \mathcal{C}^{\infty}\left(\mathbb{T}^{1}\right)$, cujos termos sejam soluções de (4.58).

Para $j \in \mathbb{Z}$ e $k=0$, obtemos as equações

$$
c(x) \partial_{x} \hat{u}(x, j, 0)+i \lambda j c(x) \hat{u}(x, j, 0)=\hat{f}(x, j, 0)
$$

para todo $x \in \mathbb{T}^{1}$. Procedendo como na demonstração da Proposição 4.17, vemos que o fato de $\lambda$ não ser um númro de Liouville implica que podemos obter uma sequência rapidamente decrescente $\hat{u}(\cdot, j, 0) \subset \mathcal{C}^{\infty}\left(\mathbb{T}^{1}\right)$ tal que cada termo $\hat{u}(\cdot, j, 0)$ é solução de (4.59).

Quando $j \in \mathbb{Z}$ e $k \in \mathbb{Z} \backslash\{0\}$, a ideia é resolver em cada intervalo $\left[x_{\ell}, x_{\ell+1}\right]$.

Pelo que foi feito nas proposições 4.17, 4.18 e 4.22, vemos que basta fixar arbitrariamente um índice $\ell \in\{1, \ldots, N\}$ e mostrar que existe uma sequência rapidamente decrescente $\hat{u}(\cdot, j, k) \subset \mathcal{C}^{\infty}\left(\left[x_{\ell}, x_{\ell+1}\right]\right)((j, k) \in \mathbb{Z} \times(\mathbb{Z} \backslash\{0\}))$ tal que cada termo $\hat{u}(\cdot, j, k)$ é solução de (4.60) e flat em $\left\{x_{\ell}, x_{\ell+1}\right\}$.

Fixado $\ell \in\{1, \ldots, N\}$, em $\left(x_{\ell}, x_{\ell+1}\right)$ a equação (4.58) torna-se

$$
\partial_{x} \hat{u}(x, j, k)+i(k / c(x)+\lambda j) \hat{u}(x, j, k)=\hat{f}(x, j, k) / c(x),
$$

para todo $(j, k) \in \mathbb{Z} \times(\mathbb{Z} \backslash\{0\})$.

No caso em que ocorre (I), as proposições 4.17 e 4.18 constroem uma sequência $\hat{u}(\cdot, j, k) \subset \mathcal{C}^{\infty}\left(\left[x_{\ell}, x_{\ell+1}\right]\right)$ com as propriedades requeridas acima.

Quando vale (II), uma sequência $\hat{u}(\cdot, j, k) \subset \mathcal{C}^{\infty}\left(\left[x_{\ell}, x_{\ell+1}\right]\right)$ satisfazendo as propriedades requeridas acima é fornecida pela Proposição 4.22 .

Para concluir a demonstração, falta analisar o que acontece quando ocorrem as situações (III) e (IV).

Suponha que ocorre (III), ou seja, que $x_{\ell} \in \mathrm{V}_{1}, x_{\ell+1} \in \mathrm{V}_{2}, b$ não muda de sinal em $\left(x_{\ell}, x_{\ell+1}\right)$ e $b \equiv 0$ em $\left(x_{\ell+1}-\delta, x_{\ell+1}\right)$, para algum $\delta>0$ suficientemente pequeno. Neste 
caso, fixe $\eta \in\left(x_{\ell}, x_{\ell+1}\right)$ e considere novamente a função

$$
\theta_{j k}(x)=i \int_{\eta}^{x}(k / c(s)+\lambda j) d s=\int_{\eta}^{x}\left(k \frac{b+i a}{a^{2}+b^{2}}(s)+i \lambda j\right) d s, x \in\left(x_{\ell}, x_{\ell+1}\right) .
$$

Assuma primeiro que $b \geqslant 0$ em $\left(x_{\ell}, x_{\ell+1}\right)$. Para $j \in \mathbb{Z}$ e $k \in \mathbb{N}$, defina

$$
\begin{aligned}
\hat{u}(x, j, k) & =\exp \left\{-\theta_{j k}(x)\right\} \int_{x_{\ell}}^{x} \exp \left\{\theta_{j k}\left(x^{\prime}\right)\right\} \hat{f}\left(x^{\prime}, j, k\right) / c\left(x^{\prime}\right) d x^{\prime} \\
& =\int_{x_{\ell}}^{x} \exp \left\{-k \int_{x^{\prime}}^{x} \frac{b+i a}{a^{2}+b^{2}}(s) d s+i \lambda j\left(x^{\prime}-x\right)\right\} \hat{f}\left(x^{\prime}, j, k\right) / c\left(x^{\prime}\right) d x^{\prime},
\end{aligned}
$$

para todo $x \in\left(x_{\ell}, x_{\ell+1}\right)$. Desta forma, obtemos uma função suave em $\left(x_{\ell}, x_{\ell+1}\right)$, a qual satisfaz (4.60).

Como $k b \geqslant 0$ e $x_{\ell} \in \mathrm{V}_{1}$, seguindo argumentos da demonstração da Proposição 4.17 , verifica-se que $\hat{u}(\cdot, j, k)$ é flat em $x_{\ell}$. Como também $x_{\ell+1} \in \mathrm{V}_{2}$ e como $b \equiv 0$ em $\left(x_{\ell+1}-\delta, x_{\ell+1}\right)$, segue que a função $\exp \left\{\theta_{j k}\right\}$ é limitada em $\left(x_{\ell}, x_{\ell+1}\right)$. Assim, a função $\exp \left\{\theta_{j k}\right\} \hat{f}(\cdot, j, k) / c$ é flat em $\left\{x_{\ell}, x_{\ell+1}\right\}$ e, portanto, para que $\hat{u}(\cdot, j, k)$ seja flat em $x_{\ell+1}$ basta que

$$
\int_{x_{\ell}}^{x_{\ell+1}} \exp \left\{\theta_{j k}(x)\right\} \hat{f}(x, j, k) / c(x) d x=0,
$$

o que é verdade, pois podemos aplicar o Lema 4.21, uma vez que $\exp \left\{\theta_{j k}\right\}$ é limitada em $\left(x_{\ell}, x_{\ell+1}\right)$ e $2 \leqslant m_{\ell}, m_{\ell+1}$.

Para $j \in \mathbb{Z}$ e $k$ um inteiro negativo $(k<0)$, defina

$$
\begin{aligned}
\hat{u}(x, j, k) & =-\exp \left\{-\theta_{j k}(x)\right\} \int_{x}^{x_{\ell+1}} \exp \left\{\theta_{j k}\left(x^{\prime}\right)\right\} \hat{f}\left(x^{\prime}, j, k\right) / c\left(x^{\prime}\right) d x^{\prime} \\
& =-\int_{x}^{x_{\ell+1}} \exp \left\{-k \int_{x^{\prime}}^{x} \frac{b+i a}{a^{2}+b^{2}}(s) d s+i \lambda j\left(x^{\prime}-x\right)\right\} \hat{f}\left(x^{\prime}, j, k\right) / c\left(x^{\prime}\right) d x^{\prime},
\end{aligned}
$$

para todo $x \in\left(x_{\ell}, x_{\ell+1}\right)$. Assim, $\hat{u}(\cdot, j, k)$ é suave em $\left(x_{\ell}, x_{\ell+1}\right)$, flat em $x_{\ell+1}$ e satisfaz (4.60). Mais ainda, utilizando as técnicas da demonstração da Proposição 4.17, verificase que $\hat{u}(\cdot, j, k)$ também é flat em $x_{\ell}$.

Portanto, no caso em que vale (III) e $b \geqslant 0$ em $\left(x_{\ell}, x_{\ell+1}\right)$, obtemos uma sequência $\hat{u}(\cdot, j, k)(k \neq 0)$ de soluções de (4.60). Precisamos, ainda, provar que tal sequência decai rapidamente. Para tanto, basta repetir as técnicas das demonstrações das proposições 4.17 e 4.22 . 
Agora, assuma que ocorre (III) e que $b \leqslant 0$ em $\left(x_{\ell}, x_{\ell+1}\right)$. Neste caso, quando $k$ é um inteiro negativo definimos $\hat{u}(\cdot, j, k)$ como em (4.61) e quando $k$ é um inteiro positivo definimos $\hat{u}(\cdot, j, k)$ como em (4.62). Note que agora $\theta_{j k}$ é limitada em $\left(x_{\ell}, x_{\ell+1}\right)$ quando $k$ é um inteiro negativo. Procedendo como acima verifica-se que a sequência $\hat{u}(\cdot, j, k)$ possui as propriedades requeridas.

Passemos à análise do caso em que ocorre (IV), isto é, $x_{\ell} \in \mathrm{V}_{2}, x_{\ell+1} \in \mathrm{V}_{1}, b$ não muda de sinal em $\left(x_{\ell}, x_{\ell+1}\right)$ e $b \equiv 0$ em $\left(x_{\ell}, x_{\ell}+\delta\right)$, para algum $\delta>0$ suficientemente pequeno. Sob tais condições, podemos construir a sequência $\hat{u}(\cdot, j, k)$ utilizando as mesmas técnicas empregadas no caso (III). Para $k \in \mathbb{Z} \backslash\{0\}$ tal que $k b \geqslant 0$, defina $\hat{u}(\cdot, j, k)$ como em (4.61) e para $k \in \mathbb{Z} \backslash\{0\}$ tal que $k b \leqslant 0$, defina $\hat{u}(\cdot, j, k)$ como em (4.62). Note que agora a função $\exp \left\{\theta_{j k}\right\}$ é limitada em $\left(x_{\ell}, x_{\ell+1}\right)$ para os índices $k$ tais que $k b \leqslant 0$. Argumentando como no caso em que ocorre (III), verifica-se que tal sequência satisfaz as propriedades requeridas, ou seja, cada termo é suave em $\left[x_{\ell}, x_{\ell+1}\right]$, flat em $\left\{x_{\ell}, x_{\ell+1}\right\}$, satisfaz (4.60) e a sequência decai rapidamente em $\left[x_{\ell}, x_{\ell+1}\right]$.

O que conclui a demonstração do Teorema 4.7. 


$\frac{-5}{5}$

\section{Considerações finais}

Em 2004, o artigo [BCP] foi pioneiro ao apresentar uma classe de campos vetoriais (complexos) em $\mathbb{T}^{2}$, os quais são fortemente resolúveis, mas não são globalmente hipoelíticos. A saber, tais campos são do tipo

$$
L=\frac{\partial}{\partial t}+i b(x, t) \frac{\partial}{\partial x}, \quad\left(b \in \mathcal{C}^{\infty}\left(\mathbb{T}^{2}, \mathbb{R}\right)\right)
$$

Depois em [BD1] e [BD2], foram exibidas mais classes de campos em $\mathbb{T}^{2}$ com tal propriedade.

Uma parte da técnica de $[\mathrm{BCP}]$ envolve uma redução que transforma o estudo da resolubilidade forte no estudo da resolubilidade em uma vizinhança de cada órbita de Sussmann unidimensional. Tais órbitas são da forma $\left\{x^{*}\right\} \times \mathbb{T}^{1}$, sendo que $b$ se anula de ordem finita $m \geqslant 2 \mathrm{em}\left\{x^{*}\right\} \times \mathbb{T}^{1}$. A resolubilidade em $\left\{x^{*}\right\} \times \mathbb{T}^{1}$ é no seguinte sentido (correlato ao de Hörmander em [H3]): existe uma vizinhança $\left(x^{*}-\delta, x^{*}+\delta\right) \times \mathbb{T}^{1}$ de $\left\{x^{*}\right\} \times \mathbb{T}^{1}$ e existe um subespaço de codimensão finita de $\mathcal{C}^{\infty}\left(\left(x^{*}-\delta, x^{*}+\delta\right) \times \mathbb{T}^{1}\right)$, $\mathfrak{F}\left(x^{*}\right)$, com a seguinte propriedade: para toda $f$ suave em uma vizinhança de $\left\{x^{*}\right\} \times \mathbb{T}^{1}$, com $f \in \mathfrak{F}\left(x^{*}\right)$, existe $u$, também suave em uma vizinhança de $\left\{x^{*}\right\} \times \mathbb{T}^{1}$, satisfazendo $L u=f$ em uma vizinhança de $\left\{x^{*}\right\} \times \mathbb{T}^{1}$.

Observe que o campo $L$ não é globalmente hipoelítico em nenhum aberto contendo o compacto $\left\{x^{*}\right\} \times \mathbb{T}^{1} ;$ mesmo assim, sob certas condições foi possível garantir a existência de solução da equação $L u=f$, impondo que $f$ satisfaça apenas um número finito de condições de compatibilidade. 
Mais recentemente, em [M], estudou-se a resolubilidade de campos vetoriais em uma vizinhança do compacto $\{0\} \times \mathbb{T}^{m} \subset \mathbb{R}^{n} \times \mathbb{T}^{m}$. Foram considerados campos vetoriais dos tipos $X+Y$ e $X+i Y$, onde

$$
X \doteq \sum_{k=1}^{m}\left(\omega_{k}+g_{k}(x, t)\right) \frac{\partial}{\partial t_{k}} \quad \text { e } \quad Y \doteq \sum_{j=1}^{n}\left(\lambda_{j} x_{j}+f_{j}(x, t)\right) \frac{\partial}{\partial x_{j}},
$$

sendo que os coeficientes satisfazem: $\omega_{k}$ e $\lambda_{j}$ são números reais e as funções $f_{j}$ e $g_{k}$ são funções reais definidas em uma vizinhança de $\{0\} \times \mathbb{T}^{m} \subset \mathbb{R}^{n} \times \mathbb{T}^{m}$, tais que $f_{j}(x, t)=$ $O\left(|x|^{2}\right)$ e $g_{j}(x, t)=O(|x|)$.

Note que tais campos não são globalmente hipoelíticos em nenhum aberto contendo o compacto $\{0\} \times \mathbb{T}^{m}$.

Para o campo $X+Y$ (caso real), mostrou-se que vale a seguinte resolubilidade (ver Teorema 2 de $[\mathrm{M}]$ ): Se $X+Y$ é de classe $C^{a}(a=\infty$ (suave) ou $a=\omega$ (analítico real)), então para toda função $F$ de classe $C^{a}$ em uma vizinhança de $\{0\} \times \mathbb{T}^{m}$ tal que $\int_{\mathbb{T}^{m}} F(0, t) d t=0$, existe $u$ de classe $C^{a}$ em uma vizinhança de $\{0\} \times \mathbb{T}^{m}$ que é solução da equação $(X+Y) u=F$ em uma vizinhança de $\{0\} \times \mathbb{T}^{m}$.

Com relação aos campos $X+i Y$, a resolubilidade no caso suave fica mais delicada. O Teorema 8.4 de $[\mathrm{M}]$ exibe uma função $f$, suave em uma vizinhança de $\{0\} \times \mathbb{T}^{m}$ e satisfazendo $\int_{\mathbb{T}^{m}} f(0, t) d t=0$, tal que a equação

$$
\left(\sum_{k=1}^{m} \omega_{k} \frac{\partial}{\partial t_{k}}+i \sum_{j=1}^{n} \lambda_{j} x_{j} \frac{\partial}{\partial x_{j}}\right) u(x, t)=f(x, t) \quad\left(\text { onde } \quad \omega_{1} \lambda_{1} \neq 0\right)
$$

não possui solução suave em nenhuma vizinhança de $\{0\} \times \mathbb{T}^{m}$.

O propósito do presente capítulo é apresentar uma nova classe de campos vetoriais com as propriedades descritas nos parágrafos acima. Mais específicamente, apresentaremos uma classe de campos vetoriais definidos em um aberto $(-\delta, \delta) \times \mathbb{T}^{2}$, os quais não são globalmente hipoelíticos em $(-\delta, \delta) \times \mathbb{T}^{2}$ e, mesmo assim, para podermos garantir a existência de solução da equação $L u=f$ bastará que $f$ satisfaça um número finito de condições de compatibilidade.

Vale lembrar que, para os operadores estudados nos capítulos 2 e 3, a resolubilidade forte e a hipoeliticidade global são equivalentes. Já os operadores do Capítulo 4, no caso em que $c^{-1}(0) \neq \emptyset$, eles não são fortemente resolúveis e também não são globalmente hipoelíticos. 


\section{I Resolubilidade perto de uma órbita compacta bidi- mensional}

Trabalharemos com campos vetoriais (complexos) em um aberto $\Omega=(-\delta, \delta) \times \mathbb{T}^{2}$ de $\mathbb{T}^{3}$, os quais são do tipo

$$
\mathfrak{L}=\frac{\partial}{\partial t}+i \beta(x)\left(x^{m} \frac{\partial}{\partial x}+\frac{\partial}{\partial y}\right)
$$

onde denotamos as coordenadas em $\Omega=(-\delta, \delta) \times \mathbb{T}^{2}$ por $(x, y, t)$.

Para estabelecer os resultados foi necessário supor algumas condições técnicas. Vamos supor que o inteiro positivo $m$ satisfaz $m \geqslant 2$ e que a função $\beta \in \mathcal{C}^{\infty}(\Omega, \mathbb{R})$ satisfaz $\beta(x)>0$, para todo $x \in(-\delta, \delta)$.

Note que $\mathfrak{L}\left(\delta(x) \otimes 1_{y} \otimes 1_{t}\right)=0$, logo $\mathfrak{L}$ não é globalmente hipoelítico em nenhuma vizinhança da órbita de Sussmann bidimensional $\{0\} \times \mathbb{T}^{2}$ (ver $[\mathrm{S}]$ ou $[\mathrm{BCH}]$ ).

Desejamos estudar a resolubilidade de $\mathfrak{L}$ perto de $\{0\} \times \mathbb{T}^{2}$, no seguinte sentido:

Definição 5.1. Diremos que $\mathfrak{L}$ é resolúvel em $\{0\} \times \mathbb{T}^{2}$ quando existir um subespaço de codimensão finita de $\mathcal{C}^{\infty}(\Omega)$ tal que, para toda $f$ nesse espaço, existe $u$, suave em uma vizinhança de $\{0\} \times \mathbb{T}^{2}$, satisfazendo Lu $=f$ em uma vizinhança de $\{0\} \times \mathbb{T}^{2}$.

Exemplo 5.2. Existem operadores definidos em $\mathbb{T}^{3}$, cuja restrição a uma vizinhança de $\{0\} \times \mathbb{T}^{2}$ possui a forma (5.1). Por exemplo, existe $b \in \mathcal{C}^{\infty}\left(\mathbb{T}^{1}, \mathbb{R}\right)$ tal que $b(x)=x^{m}$ $(m \geqslant 2)$ em uma vizinhança de $x=0, b \geqslant 0$ e $b^{-1}(0)=2 \pi \mathbb{Z}$. Assim, o operador

$$
\frac{\partial}{\partial t}+i b(x) \frac{\partial}{\partial x}+i \frac{\partial}{\partial y}
$$

é da forma (5.1).

Outro exemplo é considerar a função $1-\cos (x)$ e tomar $\phi \in \mathcal{C}^{\infty}\left(\mathbb{T}^{1}, \mathbb{R}\right)$ tal que $1-\cos (x)=x^{2} \phi(x)$, para $x \in(-\delta, \delta)$. O operador

$$
\frac{\partial}{\partial t}+i(1-\cos (x)) \frac{\partial}{\partial x}+i \phi(x) \frac{\partial}{\partial y}
$$

é da forma (5.1) em uma vizinhança de $\{0\} \times \mathbb{T}^{2}$. 
Considere $\mathfrak{F}(0) \doteq \operatorname{ker}^{t} \mathfrak{L} \cap \mathcal{E}^{\prime}\left(\{0\} \times \mathbb{T}^{2}\right) \subset \mathcal{E}^{\prime}(\Omega)$. Um cálculo direto mostra que $\delta^{(\ell)}(x) \otimes 1_{y} \otimes 1_{t} \in \mathfrak{F}(0)$, para cada $\ell=0, \ldots, m-1$. Assim,

$$
\begin{aligned}
& \mathfrak{F}(0)^{\circ} \subset\left\{f \in \mathcal{C}^{\infty}(\Omega) ;\left\langle\delta^{(\ell)}(x) \otimes 1_{y} \otimes 1_{t}, f\right\rangle=0, \text { para todo } \ell=0, \ldots, m-1\right\} \\
& =\left\{f \in \mathcal{C}^{\infty}(\Omega) ; \int_{0}^{2 \pi} \int_{0}^{2 \pi} \partial_{x}^{\ell} f(0, y, t) d y d t=0, \text { para todo } \ell=0, \ldots, m-1\right\} .
\end{aligned}
$$

Veremos que $\mathfrak{F}(0)$ é gerado pelas distribuições $\delta^{(\ell)}(x) \otimes 1_{y} \otimes 1_{t}, \ell=0, \ldots, m-1$. Em particular, seguirá que a inclusão acima é de fato uma igualdade e que $\mathfrak{F}(0)^{\circ}$ é um subespaço de codimensão finita $(m)$ de $\mathcal{C}^{\infty}(\Omega)$, já que $\operatorname{dim} \mathfrak{F}(0)=m$ implica que $\operatorname{dim} \mathcal{C}^{\infty}(\Omega) / \mathfrak{F}(0)^{\circ}=\operatorname{dim} \mathfrak{F}(0)=m$.

Mostraremos também que dada $f \in \mathfrak{F}(0)^{\circ}$, existe $u$ suave em uma vizinhança de $\{0\} \times \mathbb{T}^{2}$ tal que $\mathfrak{L} u=f$ em uma vizinhança de $\{0\} \times \mathbb{T}^{2}$. Portanto, $\mathfrak{L}$ é resolúvel (conforme a Definição 5.1) em $\{0\} \times \mathbb{T}^{2}$, embora não seja globalmente hipoelítico em nenhuma vizinhança de $\{0\} \times \mathbb{T}^{2}$.

Teorema 5.3. $\mathfrak{F}(0)^{\circ}$ é um subespaço de codimensão finita de $\mathcal{C}^{\infty}(\Omega)$. Para cada $f \in$ $\mathfrak{F}(0)^{\circ}$ e $0<\sigma<\delta$, existe $u \in \mathcal{C}^{\infty}\left((-\sigma, \sigma) \times \mathbb{T}^{2}\right)$ tal que $\mathfrak{L} u=f$ em $(-\sigma, \sigma) \times \mathbb{T}^{2}$.

A primeira etapa será mostrar que $\mathfrak{F}(0)$ tem dimensão finita e que vale a redução módulo flat.

Proposição 5.4. Se $f \in \mathcal{C}^{\infty}(\Omega)$ e se $\left\langle\delta^{(\ell)}(x) \otimes 1_{y} \otimes 1_{t}, f\right\rangle=0$, para $\ell=0, \ldots, m-1$, então existe $u \in \mathcal{C}^{\infty}(\Omega)$ tal que $\mathfrak{L} u-f$ é flat em $\{0\} \times \mathbb{T}^{2}$. Além disso, $\mathfrak{F}(0)=\operatorname{ker}^{t} \mathfrak{L} \cap$ $\mathcal{E}^{\prime}\left(\{0\} \times \mathbb{T}^{2}\right)$ é gerado pelas distribuições $\delta^{(\ell)}(x) \otimes 1_{y} \otimes 1_{t}, \ell=1, \ldots, m-1$.

Demonstração: Mostraremos inicialmente a existência da função $u$, onde procederemos como no Lema 4.16 (ver também Proposição 4.1 de [BCP]). Utilizando as expressões formais das séries de Taylor de $u, f$ e $\beta$, escrevemos

$$
\begin{aligned}
\beta(x) & \sim \beta_{0}+\beta_{1} x^{1}+\beta_{2} x^{2}+\cdots, \quad \text { onde } \beta_{0} \neq 0, \\
u(x, y, t) & \sim u_{0}(y, t)+u_{1}(y, t) x+u_{2}(y, t) x^{2}+\cdots
\end{aligned}
$$

e

$$
f(x, y, t) \sim f_{0}(y, t)+f_{1}(y, t) x+f_{2}(y, t) x^{2}+\cdots
$$

A equação $\mathfrak{L} u-f \sim 0$ é equivalente às seguintes equações: 


$$
\begin{gathered}
\partial_{t} u_{j}(y, t)+i \sum_{\ell=0}^{j} \beta_{j-\ell} \partial_{y} u_{\ell}(y, t)=f_{j}(y, t), \quad \text { onde } \quad j=0, \ldots, m-1 . \\
\partial_{t} u_{m}(y, t)+i \beta_{0} u_{1}(y, t)+i \sum_{\ell=0}^{m} \beta_{m-\ell} \partial_{y} u_{\ell}(y, t)=f_{m}(y, t) . \\
\partial_{t} u_{m+k}+i \sum_{\ell=0}^{k} \beta_{\ell}(k+1-\ell) u_{k+1-\ell}+i \sum_{\ell=0}^{m+k} \beta_{m+k-\ell} \partial_{y} u_{\ell}=f_{m+k}, \quad \text { para todo } \quad k \geqslant 1 .
\end{gathered}
$$

Para cada $j=0, \ldots, m-1$, podemos utilizar série de Fourier nas variáveis $(y, t)$ e proceder como na demonstração da Proposição 2.17 para resolver cada equação em (5.2), pois são equações com coeficientes constantes da forma

$$
\partial_{t} u_{j}(y, t)+i \beta_{0} \partial_{y} u_{j}(y, t)=f_{j}(y, t)-i \sum_{\ell=0}^{j-1} \beta_{j-\ell} \partial_{y} u_{\ell}(y, t)
$$

onde $\beta_{0}>0$

$$
\sum_{\ell=1}^{j} \beta_{j-\ell} \int_{0}^{2 \pi} \int_{0}^{2 \pi} \partial_{y} u_{\ell}(y, t) d y d t=0
$$

e, por hipótese,

$$
\int_{0}^{2 \pi} \int_{0}^{2 \pi} f_{j}(y, t) d y d t=\int_{0}^{2 \pi} \int_{0}^{2 \pi} \partial_{x}^{j} f(0, y, t) d y d t=0
$$

Note que, para cada $C_{j} \in \mathbb{C}$, a função $u_{j}+C_{j}$ é ainda uma solução de (5.2). Cada constante $C_{j}$ será fixada apropriadamente.

A equação (5.3) pode ser escrita na forma

$$
\partial_{t} u_{m}(y, t)+i \beta_{0} \partial_{y} u_{m}(y, t)=f_{m}(y, t)-i \beta_{0} u_{1}(y, t)-i \sum_{\ell=0}^{m-1} \beta_{m-\ell} \partial_{y} u_{\ell}(y, t)
$$

e como $m \geqslant 2$, estamos novamente com uma equação do tipo (5.5). A hipótese de que $\beta_{0}>0$ implica que podemos fixar uma constante $C_{1}$, de modo que a equação (5.3) tenha solução; a saber,

$$
C_{1} \doteq-i\left(4 \pi^{2} \beta_{0}\right)^{-1} \int_{0}^{2 \pi} \int_{0}^{2 \pi}\left[f_{m}-i \beta_{0} u_{1}\right] d y d t
$$


Novamente, após fixarmos uma solução de (5.3), $u_{m}$, para cada $C_{m} \in \mathbb{C}$, a função $u_{m}+C_{m}$ é ainda uma solução de (5.3). A constante $C_{m}$ também será fixada apropriadamente, como fizemos com $C_{1}$.

Repetindo esse processo de maneira indutiva, verifica-se que fixando apropriadamente as constantes $C_{2}, \ldots, C_{m}, C_{m+1}, \ldots, C_{m+k+1}, \ldots$, obtemos soluções para as equações do tipo (5.4).

Por fim, como no Lema 4.16, recorremos ao Lema de Borel para produzir uma função $u \in \mathcal{C}^{\infty}\left((-\delta, \delta) \times \mathbb{T}^{2}\right)$, tal que $\mathfrak{L} u-f$ é flat em $\{0\} \times \mathbb{T}^{2}$.

O que finaliza a redução módulo flat.

Utilizaremos essa primeira parte para mostrar que $\mathfrak{F}(0)$ é gerado pelas distribuições $\delta^{(\ell)}(x) \otimes 1_{y} \otimes 1_{t}, \ell=0, \ldots, m-1$, onde recordamos que $\mathfrak{F}(0)=\operatorname{ker}^{t} \mathfrak{L} \cap \mathcal{E}^{\prime}\left(\{0\} \times \mathbb{T}^{2}\right)$.

Suponha, por contradição, que existe $\mu \in \mathfrak{F}(0)$ tal que $\mu$ não é uma combinação linear das distribuições $\delta^{(\ell)}(x) \otimes 1_{y} \otimes 1_{t}, \ell=0, \ldots, m-1$. Pelo Teorema de Hahn-Banach, existe $f \in \mathcal{C}^{\infty}(\Omega)$ tal que $\langle\mu, f\rangle \neq 0$ e $\left\langle\delta^{(\ell)}(x) \otimes 1_{y} \otimes 1_{t}, f\right\rangle=0$, para $\ell=0, \ldots, m-1$. Segue da redução módulo flat que existe $u \in \mathcal{C}^{\infty}(\Omega)$ tal que $g \doteq \mathfrak{L} u-f$ é flat em $\{0\} \times \mathbb{T}^{2}$. Como supp $\mu \subset\{0\} \times \mathbb{T}^{2}$ e ${ }^{t} \mathfrak{L} \mu=0$, obtemos

$$
0=\langle\mu, g\rangle=\langle\mu, \mathfrak{L} u-f\rangle=\left\langle{ }^{t} \mathfrak{L} \mu, u\right\rangle-\langle\mu, f\rangle=-\langle\mu, f\rangle
$$

o que é uma contradição.

A demonstração da Proposição 5.4 está completa.

O próximo passo será seguir a linha de [BD1] para mostrar que é possível construir uma solução para a equação $\mathfrak{L} u=f$, para toda $f \in \mathfrak{F}(0)^{\circ}$ que é flat em $\{0\} \times \mathbb{T}^{2}$.

Proposição 5.5. Seja $\mathfrak{L}$ o operador dado por (5.1). Para cada $f \in \mathfrak{F}(0)^{\circ}, f$ flat em $\{0\} \times \mathbb{T}^{2}$, e para cada $0<\sigma<\delta$, existe $u \in \mathcal{C}^{\infty}\left((-\sigma, \sigma) \times \mathbb{T}^{2}\right)$ tal que $\mathfrak{L} u=f$ em $(-\sigma, \sigma) \times \mathbb{T}^{2}$.

Demonstração: Dada $f \in \mathfrak{F}(0)^{\circ} \subset \mathcal{C}^{\infty}\left((-\delta, \delta) \times \mathbb{T}^{2}\right), f$ flat em $\{0\} \times \mathbb{T}^{2}$, e dado $0<\sigma<\delta$, vamos encontrar $u \in \mathcal{C}^{\infty}\left((-\sigma, \sigma) \times \mathbb{T}^{2}\right)$, de modo que $u$ seja flat em $\{0\} \times \mathbb{T}^{2}$ e satisfaça a equação $\mathfrak{L} u=f$ em $(-\sigma, \sigma) \times \mathbb{T}^{2}$.

Como o coeficiente $\beta$ do operador $\mathfrak{L}$ não depende das variáveis $y$ e $t$, podemos utilizar série parcial de Fourier para simplificar a equação $\mathfrak{L} u=f$. 
Escrevendo

$$
u(x, y, t)=\sum_{(j, k) \in \mathbb{Z}^{2}} \hat{u}(x, j, k) e^{i(j y+k t)}
$$

e

$$
f(x, y, t)=\sum_{(j, k) \in \mathbb{Z}^{2}} \hat{f}(x, j, k) e^{i(j y+k t)}
$$

basta encontrar uma sequência de funções rapidamente decrescente, $(\hat{u}(\cdot, j, k))$, tal que cada termo $\hat{u}(\cdot, j, k)$ seja solução da equação

$$
i \beta(x) x^{m} \partial_{x} \hat{u}(x, j, k)+(i k-j \beta(x)) \hat{u}(x, j, k)=\hat{f}(x, j, k),
$$

onde $x \in(-\delta, \delta)$ e $(j, k) \in \mathbb{Z}^{2}$.

Vamos construir $u \in \mathcal{C}^{\infty}\left([0, \sigma) \times \mathbb{T}^{2}\right)$. A construção de $u$ em $(-\sigma, 0] \times \mathbb{T}^{2}$ é feita de maneira análoga.

No intervalo $(0, \sigma)$, a equação (5.6) fica equivalente a

$$
\partial_{x} \hat{u}(x, j, k)+\left(\frac{k}{\beta(x) x^{m}}+\frac{i j}{x^{m}}\right) \hat{u}(x, j, k)=\frac{\hat{f}(x, j, k)}{i \beta(x) x^{m}} .
$$

Fixado $\eta \in(0, \sigma)$, defina

$$
\theta_{j k}(x)=\int_{\eta}^{x}\left(\frac{k}{\beta(s) s^{m}}+\frac{i j}{s^{m}}\right) d s, x \in(0, \sigma)
$$

Como $f$ é flat em $\{0\} \times \mathbb{T}^{2}$, podemos considerar $g(x, r, t) \doteq f(x, r, t) / i \beta(x) x^{m}$, obtendo $g \in \mathcal{C}^{\infty}\left((-\delta, \delta) \times \mathbb{T}^{2}\right)$; mais ainda, $g$ é flat em $\{0\} \times \mathbb{T}^{2}$ e seus coeficientes parciais de Fourier satisfazem $\hat{g}(x, j, k)=\hat{f}(x, j, k) / i \beta(x) x^{m}$, para todo $(j, k) \in \mathbb{Z}^{2}$.

Para $k \geqslant 0$, consideraremos a solução de (5.7) que é dada por

$$
\hat{u}(x, j, k)=\int_{0}^{x} \exp \left\{\theta_{j k}\left(x^{\prime}\right)-\theta_{j k}(x)\right\} \hat{g}\left(x^{\prime}, j, k\right) d x^{\prime}, \text { para todo } x \in(0, \sigma) .
$$

A função acima está bem definida em $(0, \sigma)$, pois para cada $x^{\prime} \in(0, \eta)$, temos

$$
\mid \exp \left\{\theta_{j k}\left(x^{\prime}\right) \mid=\exp \left\{-k \int_{x^{\prime}}^{\eta} \frac{1}{\beta(s) s^{m}} d s\right\} \leqslant 1\right.
$$

uma vez que $\beta>0$ e $k \geqslant 0$. Mais ainda, o Teorema Fundamental do Cálculo implica que $\hat{u}(\cdot, j, k)$ é infinitamente diferenciável em $(0, \sigma)$ e que $\hat{u}(\cdot, j, k)$ é solução de $(5.7)$. 
Afirmamos que $\hat{u}(\cdot, j, k)$ é flat em $x=0$. De fato, como

$$
\left|\exp \left\{\theta_{j k}\left(x^{\prime}\right)-\theta_{j k}(x)\right\}\right|=\exp \left\{-\int_{x^{\prime}}^{x} \frac{k}{\beta(s) s^{m}} d s\right\} \leqslant 1, \quad \text { para todo } \quad x^{\prime} \in(0, x),
$$

obtemos

$$
|\hat{u}(x, j, k)| \leqslant \int_{0}^{x}\left|\hat{g}\left(x^{\prime}, j, k\right)\right| d x^{\prime} \leqslant \sup _{\left(x^{\prime}, r, t\right) \in[0, x] \times \mathbb{T}^{2}}\left|g\left(x^{\prime}, r, t\right)\right| x .
$$

Como $g$ é flat em $\{0\} \times \mathbb{T}^{2}$, para cada $n \in \mathbb{Z}_{+}$, obtemos $C_{n}>0$, a qual não depende de $(j, k)$, tal que

$$
|\hat{u}(x, j, k)| \leqslant C_{n} x^{n+1}
$$

Utilizando a equação (5.7) e um processo recursivo, verifica-se que vale o mesmo para cada derivada $\partial_{x}^{\ell} \hat{u}(\cdot, j, k)$. Em particular, $\hat{u}(\cdot, j, k)$ é flat em $x=0$.

Agora vamos construir soluções para as equações (5.7), considerando os índices $k<0$.

Quando $k<0$, fixamos a solução

$$
\hat{u}(x, j, k)=-\int_{x}^{\sigma} \exp \left\{\theta_{j k}\left(x^{\prime}\right)-\theta_{j k}(x)\right\} \hat{g}\left(x^{\prime}, j, k\right) d x^{\prime}, \text { para todo } x \in(0, \sigma)
$$

a qual está bem definida, é infinitamente diferenciável em $(0, \sigma)$ e satisfaz a equação (5.7).

O próximo passo será mostrar que $\hat{u}(\cdot, j, k)$ é flat em $x=0$, para todo $k<0$.

Para $x \in(0, \sigma / 2)$, temos

$$
\begin{gathered}
|\hat{u}(x, j, k)| \leqslant \int_{x}^{2 x} \exp \left\{-\int_{x^{\prime}}^{x} \frac{k}{\beta(s) s^{m}} d s\right\}\left|\hat{g}\left(x^{\prime}, j, k\right)\right| d x^{\prime}+ \\
\int_{2 x}^{\sigma} \exp \left\{-\int_{x^{\prime}}^{x} \frac{k}{\beta(s) s^{m}} d s\right\}\left|\hat{g}\left(x^{\prime}, j, k\right)\right| d x^{\prime} .
\end{gathered}
$$

A primeira das integrais acima satisfaz

$$
\begin{gathered}
\int_{x}^{2 x} \exp \left\{-\int_{x^{\prime}}^{x} \frac{k}{\beta(s) s^{m}} d s\right\}\left|\hat{g}\left(x^{\prime}, j, k\right)\right| d x^{\prime} \leqslant \int_{x}^{2 x}\left|\hat{g}\left(x^{\prime}, j, k\right)\right| d x^{\prime} \leqslant \\
\sup _{\left(x^{\prime}, r, t\right) \in[x, 2 x] \times \mathbb{T}^{2}}\left|g\left(x^{\prime}, r, t\right)\right| x \leqslant C_{n} x^{n+1},
\end{gathered}
$$

para todo $n \in \mathbb{Z}_{+}$, onde $C_{n}$ não depende de $(j, k)$. 
A segunda integral satisfaz

$$
\begin{gathered}
\int_{2 x}^{\sigma} \exp \left\{-\int_{x^{\prime}}^{x} \frac{k}{\beta(s) s^{m}} d s\right\}\left|\hat{g}\left(x^{\prime}, j, k\right)\right| d x^{\prime}= \\
\int_{2 x}^{\sigma} \exp \left\{-\int_{x^{\prime}}^{2 x} \frac{k}{\beta(s) s^{m}} d s-\int_{2 x}^{x} \frac{k}{\beta(s) s^{m}} d s\right\}\left|\hat{g}\left(x^{\prime}, j, k\right)\right| d x^{\prime} \leqslant \\
\left(\sup _{\left(x^{\prime}, r, t\right) \in[0, \sigma] \times \mathbb{T}^{2}}\left|g\left(x^{\prime}, r, t\right)\right|\right) \sigma \exp \left\{-\int_{2 x}^{x} \frac{k}{\beta(s) s^{m}} d s\right\} \leqslant \\
\left.\sup _{\left(x^{\prime}, r, t\right) \in[0, \sigma] \times \mathbb{T}^{2}}\left|g\left(x^{\prime}, r, t\right)\right|\right) \sigma \exp \left\{\left(\inf _{s \in[0, \sigma]} \frac{1}{\beta(s)}\right) k \frac{\left(1-2^{1-m}\right)}{m-1} x^{1-m}\right\} \leqslant \\
M_{1} \exp \left\{-M_{2} \frac{\left(1-2^{1-m}\right)}{m-1} x^{1-m}\right\},
\end{gathered}
$$

onde $M_{1}$ e $M_{2}$ são constantes positivas que não dependem de $(j, k)$.

Novamente, com um processo recursivo envolvendo a equação (5.7) verifica-se que cada derivada $\partial_{x}^{\ell} \hat{u}(\cdot, j, k)$ satisfaz estimativas como (5.9) e (5.10). Em particular, $\hat{u}(\cdot, j, k)$ é flat em $x=0$.

Por fim, como foi feito nos resultados do Capítulo 4, o fato de que as estimativas (5.8), $(5.9)$ e $(5.10)$ são independentes de $(j, k)$, junto com o decaimento rápido de $\hat{g}(\cdot, j, k)$, implicam que $\hat{u}(\cdot, j, k)$ decai rapidamente.

O que conclui a demonstração da Proposição 5.5.

As proposições 5.4 e 5.5 demonstram o Teorema 5.3. De fato, para cada $g \in \mathfrak{F}(0)^{\circ}$, pela redução feita na Proposição 5.4 existe $v$ tal que $\mathfrak{L} v-g$ é flat em $\{0\} \times \mathbb{T}^{2}$. Como $f \doteq \mathfrak{L} v-g$ pertence a $\mathfrak{F}(0)^{\circ}$, segue da Proposição 5.5 que podemos tomar $w$ tal que $\mathfrak{L} w=f=\mathfrak{L} v-g$ em uma vizinhança de $\{0\} \times \mathbb{T}^{2} ;$ assim, fazendo $u \doteq v-w$, obtemos $\mathfrak{L} u=g$ em uma vizinhança de $\{0\} \times \mathbb{T}^{2}$.

Para concluir este capítulo, faremos uma análise sobre a resolubilidade global dos operadores $\left(\mathfrak{L}: \mathcal{C}^{\infty}\left(\mathbb{T}^{3}\right) \rightarrow \mathcal{C}^{\infty}\left(\mathbb{T}^{3}\right)\right.$ ) do Exemplo 5.2. Note que a restrição de tais operadores a uma vizinhança da órbita bidimensional $\{0\} \times \mathbb{T}^{2}$ é da forma (5.1). Se $f \in\left(\operatorname{ker}^{t} \mathfrak{L}\right)^{\circ} \subset \mathcal{C}^{\infty}\left(\mathbb{T}^{3}\right)\left({ }^{t} \mathfrak{L}: \mathcal{D}^{\prime}\left(\mathbb{T}^{3}\right) \rightarrow \mathcal{D}^{\prime}\left(\mathbb{T}^{3}\right)\right)$, então repetindo as técnicas utilizadas no Capítulo 4, podemos obter uma função $u \in \mathcal{C}^{\infty}\left(\mathbb{T}^{3}\right)$ tal que $\mathfrak{L} u=f$. Portanto, a imagem do operador $\mathfrak{L}$ é um subespaço fechado de $\mathcal{C}^{\infty}\left(\mathbb{T}^{3}\right)$. Porém, veremos que a imagem de $\mathfrak{L}$ não tem codimensão finita. 
Considere

$$
\mathfrak{L}=\frac{\partial}{\partial t}+i b(x) \frac{\partial}{\partial x}+i \frac{\partial}{\partial y},
$$

onde $b$ é como no Exemplo 5.2, isto é, $b \in \mathcal{C}^{\infty}\left(\mathbb{T}^{1}, \mathbb{R}\right), b(x)=x^{m}(m \geqslant 2)$ em uma vizinhança de $x=0, b \geqslant 0$ e $b^{-1}(0)=0 \in \mathbb{T}^{1}$.

Fixado $\eta \in(0,2 \pi)$ e tomando

$$
\psi_{j}(x)=\exp \left\{-j i \int_{\eta}^{x} b^{-1}(s) d s\right\}, \quad j \neq 0 \text { e } x \in(0,2 \pi)
$$

obtemos $\psi_{j} \in \mathrm{L}^{\infty}\left(\mathbb{T}^{1}\right) \subset \mathcal{D}^{\prime}\left(\mathbb{T}^{1}\right)$. Como $b$ se anula apenas de ordem finita, existe $\psi_{j} / b \in$ $\mathcal{D}^{\prime}\left(\mathbb{T}^{1}\right)$ tal que $b\left(\psi_{j} / b\right)=\psi_{j}$. Considerando o operador

$$
{ }^{t} \mathfrak{L}_{j}: \mathcal{D}^{\prime}\left(\mathbb{T}^{1}\right) \rightarrow \mathcal{D}^{\prime}\left(\mathbb{T}^{1}\right) \quad \text { dado por } \quad{ }^{t} \mathfrak{L}_{j}(\mu)=i \frac{d}{d x}(b \mu)-j \mu
$$

e definindo $\omega_{j}={ }^{t} \mathfrak{L}_{j}\left(\psi_{j} / b\right)$, obtemos $\operatorname{supp} \omega_{j} \subset\{0\} \subset \mathbb{T}^{1}$. Procedendo como na demonstração do Lema 4.21 (ver também Proposição 2.25 em [G]), verifica-se que existe $\nu_{j} \in \mathcal{D}^{\prime}\left(\mathbb{T}^{1}\right)$ tal que $\omega_{j}={ }^{t} \mathfrak{L}\left(\nu_{j}\right)$, onde $\nu_{j}$ é da forma $\nu_{j}=\sum_{\ell=0}^{r} \alpha_{\ell} \delta^{(\ell)}(x)$. Em particular, ${ }^{t} \mathfrak{L}\left(\psi_{j} / b-\nu_{j}\right)=0$. Um cálculo direto mostra que as distribuições $\mu_{j} \doteq\left(\psi_{j} / b-\nu_{j}\right) \otimes e^{i j y}$ estão no núcleo de ${ }^{t} \mathfrak{L}$. Assim, segue do Lema 1.1 que a imagem do operador $\mathfrak{L}$ tem codimensão infinita.

Agora considere o outro operador do Exemplo 5.2,

$$
\frac{\partial}{\partial t}+i(1-\cos (x)) \frac{\partial}{\partial x}+i \phi(x) \frac{\partial}{\partial y}
$$

onde $\phi \in \mathcal{C}^{\infty}\left(\mathbb{T}^{1}, \mathbb{R}\right)$ e $1-\cos (x)=x^{2} \phi(x)$, em uma vizinhança de $x=0$. Para verificar que tal operador também tem imagem de codimensão infinita, repetimos a mesma técnica, considerando

$$
\psi_{j}=\exp \left\{-j i \int_{\eta}^{x} \phi(s)[1-\cos (s)]^{-1} d s\right\}, \quad x \in(0,2 \pi)
$$

e

$$
{ }^{t} \mathfrak{L}_{j}(\mu)=i \frac{d}{d x}((1-\cos (x)) \mu)-j \phi(x) \mu
$$




\section{Referências Bibliográficas}

[B] A. P. Bergamasco. Remarks about global analytic hypoellipticity. Trans. Amer. Math. Soc., 351(10):4113-4126, 1999.

[BCH] S. Berhanu, P. D. Cordaro, and J. Hounie. An introduction to involutive structures, volume 6 of New Mathematical Monographs. Cambridge University Press, Cambridge, 2008.

[BCP $]$ A. P. Bergamasco, P. D. Cordaro, and G. Petronilho. Global solvability for a class of complex vector fields on the two-torus. Comm. Partial Differential Equations, 29:785-819, 2004.

[BD1] A. P. Bergamasco and P. L. Dattori da Silva. Global solvability for a special class of vector fields on the torus. Contemp. Math., 400:11-20, 2006.

[BD2] A. P. Bergamasco and P. L. Dattori da Silva. Solvability in the large for a class of vector fields on the torus. J. Math. Pures Appl., 86(5):427-447, 2006.

[BD3] A. P. Bergamasco and P. L. Dattori da Silva. Solvability in the large for a class of complex vector fields on the cylinder. Bull. Sci. Math., 136(2):162$171,2012$.

[BDM] A. P. Bergamasco, P. L. Dattori da Silva, and A. Meziani. Solvability of a first order differential operator on the two-torus. J. Math. Anal. Appl., 416(1):166-180, 2014.

[BF $] \quad$ R. Beals and C. Fefferman. On local solvability of linear partial differential equations. Ann. of Math, 97:482-498, 1973.

[BP] A. P. Bergamasco and G. Petronilho. Closedness of the range for vector fields on the torus. J. Differential Equations, 154(1):132-139, 1999.

[C] P. D. Cordaro. Resolubilidade das equações diferenciais parciais lineares. Matemática Universitária, 14:51-67, 1992. 
[CHo] F. Cardoso and J Hounie. Global solvability of an abstract complex. Proc. Amer. Math. Soc., 65:117-124, 1977.

[D1] P. L. Dattori da Silva. Resolubilidade global para uma classe de campos vetoriais no toro. Tese de Doutorado, UFSCAR, São Carlos - SP, 2004.

[D2] P. L. Dattori da Silva. Nonexistence of global solutions for a class of complex vector fields on two-torus. J. Math. Anal. Appl., 351(2):543-555, 2009.

[G] R. B. Gonzalez. Resolubilidade global de uma classe de campos vetoriais. Dissertação de Mestrado, ICMC-USP, São Carlos - SP, 2010.

[GW] S. J. Greenfield and N. R. Wallach. Global hypoellipticity and Liouville numbers. Proc. Amer. Math. Soc., 31:112-114, 1972.

[H1] L. Hörmander. Differential equations without solutions. Math. Ann., 140:169-173, 1960.

[H2] L. Hörmander. Linear partial differential operators. Academic Press, Inc., Publishers, New York; Springer-Verlag, Berlin-Göttingen-Heidelberg, 1963.

[H3] L. Hörmander. Propagation of singularities and semiglobal existence theorems for (pseudo)differential operators of principal type. Ann. of Math. (2), 108(3):569-609, 1978.

[H4] Lars Hörmander. Pseudodifferential operators of principal type, volume 65 of Singularities in boundary value problems (Proc. NATO Adv. Study Inst., Maratea, 1980). Reidel, Dordrecht-Boston, Mass., 1981.

[H5] L. Hörmander. The analysis of linear partial differential operators I. SpringerVerlag, Berlin, 1983.

[H6] L. Hörmander. The analysis of linear partial differential operators IV. Springer-Verlag, Berlin, 1985.

[Ho] J. Hounie. Globally hypoelliptic and globally solvable first-order evolution equations. Trans. Amer. Math. Soc., 252:233-248, 1979.

[K] A. Kirilov. Algumas observações sobre a hipoeliticidade global no toro ndimensional. Dissertação de Mestrado - UFSCAR, 1996. 
[M] A. Meziani. Normalization and solvability of vector fields near trapped orbits. to appear.

[NT] L. Nirenberg and F. Treves. Solvability of a first order linear partial differential equation. Comm. Pure Appl. Math., 16:331-351, 1963.

[R] W. Rudin. Functional analysis. International Series in Pure and Applied Mathematics. McGraw-Hill, Inc., New York, second edition, 1991.

[S] H. J. Sussmann. Orbits of families of vector fields and integrability of distributions. Trans. Amer. Math. Soc., 180:171-188, 1973.

[T1] F. Treves. Study of a model in the theory of complexes of pseudodifferential operators. Ann. of Math. (2), 104(2):269-324, 1976.

[T2] F. Treves. Integral representation of solutions of first order linear partial differential equations. Ann. Scuola Norm., 3:1-35, 1976.

[T3] F. Treves. Topological vector spaces, distributions and kernels. Academic Press, New York, 1967.

[Z] S. L. Zani. Hipoeliticidade global para operadores de segunda ordem. Dissertação de Mestrado, ICMC-USP, São Carlos - SP, 1988. 